Supporting Information

\title{
A New Approach to Non-coordinating Anions: Lewis Acid Enhancement of Porphyrin Metal Centers in a Zwitterionic Metal-Organic Framework
}

\author{
Jacob A. Johnson, ${ }^{\dagger}$ Brenna M. Petersen, ${ }^{\dagger}$ Attila Kormos ${ }^{\ddagger}$, Elena Echeverría, ${ }^{\#}$ Yu-Sheng Chen, ${ }^{\S}$ and \\ Jian Zhang *,+ \\ ${ }^{\dagger}$ Department of Chemistry, University of Nebraska-Lincoln, Lincoln, Nebraska, 68588-0304, \\ United States \\ ${ }^{\ddagger}$ Chemical Biology Research Group, Institute of Organic Chemistry, Research Centre for Natural \\ Sciences, Hungarian Academy of Sciences, Magyar tudósok krt. 2, H-1117, Budapest, Hungary \\ ${ }^{\S}$ ChemMatCARS, Center for Advanced Radiation Sources, The University of Chicago, 9700 S. Cass \\ Avenue, Argonne, Illinois, 60439, United States \\ \#Department of Physics and Astronomy, Theodore Jorgensen Hall, $855 \mathrm{~N}$ 16th St., University of \\ Nebraska, Lincoln, Nebraska 68588-0299, United States \\ E-mail: jzhang3@unl.edu
}




\section{Table of Contents}

Section S1 Materials and General Procedures $\quad$ S3

Section S2 Synthesis and Characterization of UNLPF-13, -14, -15, and -16 S4

Section S3 [2+1] Enyne Cycloisomerization Reaction S22

Section S4 [3+2] Cycloaddition Reaction $\quad$ S34

Section S5 [4+2] Hetero-Diels-Alder Reaction S53

Section S6 ${ }^{1} H$ NMR and ${ }^{13}$ C NMR Spectra $\quad$ S60

Section S7 References $\quad$ S91 


\section{Section S1 Materials and General Procedures}

All solvents and reagents were purchased and commercially available and, unless otherwise noted, used without further purification. ${ }^{1} \mathrm{H} N M R,{ }^{13} \mathrm{C} \mathrm{NMR},{ }^{19} \mathrm{~F}$ NMR were performed on a Bruker Avance III-HD NMR spectrometer ( $300 \mathrm{MHz}$ or $400 \mathrm{MHz}$ ). Mass spectrometry was performed on a Waters Q-TOF I mass spectrometer. UV-Vis adsorption measurements were made using an Agilent Cary 300 UV-Vis spectrometer. Powder X-ray diffraction data was collected with a Panalytical Empyrean diffractometer using a rotating sample mount. The copper target X-ray tube was set to $40 \mathrm{kV}$ and $40 \mathrm{~mA}$.X-ray photoemission spectroscopy (XPS) spectra were acquired with a dual anode X-ray lamp and a hemispherical angle resolved electron analyzer (detector). Samples were measured inside an ultra-high vacuum (UHV) chamber, about $10^{-10}$ Torr, to prevent impurity scattering events. The X-ray source used was the Mg-K $\alpha$ line at $1253.6 \mathrm{eV}$, with data taken at normal emission. The XPS data were extracted and analyzed utilizing the CASA software package. Inductively coupled plasma mass spectrometry was performed using an Agilent 7500cx ICP-MS. Thermogravimetric Analysis (TGA) was performed on a Perkin Elmer STA 6000. 
Section S2 Synthesis and Characterization of UNLPF-13, -14, -15, and -16

\section{S2.1 Synthesis and Characterization of free-base porphyrin 1g}

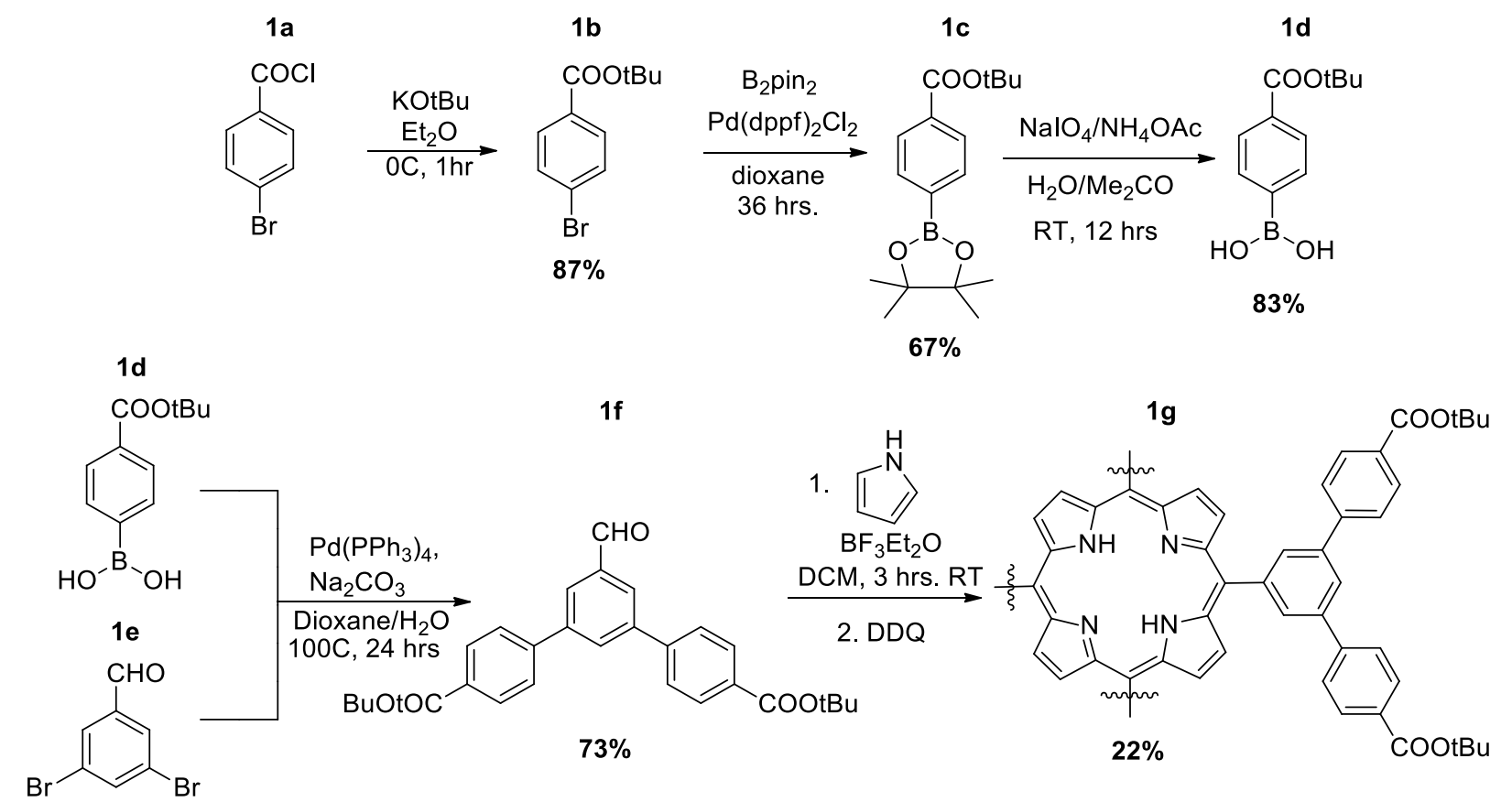

Scheme S1. Synthesis of porphyrin ligand 1g.

\section{Tert-butyl 4-bromobenzoate (1b).}

A suspension of potassium tert-butoxide $(7.6 \mathrm{~g}, 63 \mathrm{mmol})$ in ethyl ether $(67 \mathrm{~mL})$ was added dropwise to a solution of 4-bromobenzoyl chloride (1a) $(10.0 \mathrm{~g}, 46 \mathrm{mmol})$ in ethyl ether $(112 \mathrm{~mL})$ at $0^{\circ} \mathrm{C}$. The reaction was stirred for 1 hour on the ice bath and the washed with saturated $\mathrm{NaHCO}_{3}$ and brine. The organic layer was dried over $\mathrm{Na}_{2} \mathrm{SO}_{4}$, purified by column chromatotgraphy with an elution of hexanes/ethyl acetate (v/v 10:1), and further dried in vacuo to give the desired product as a pale yellow oil. Yield: 10.2 g, $87 \%$.

${ }^{1} \mathrm{HNMR}\left(400 \mathrm{MHz}, \mathrm{CDCl}_{3}\right) \delta 7.87(\mathrm{~d}, J=8.8 \mathrm{~Hz}, 2 \mathrm{H}), 7.56(\mathrm{~d}, J=8.8 \mathrm{~Hz}, 2 \mathrm{H}), 1.60(\mathrm{~s}, 9 \mathrm{H})$.

${ }^{13} \mathrm{C}$ NMR $\left(100 \mathrm{MHz}, \mathrm{CDCl}_{3}\right) \delta 165.07,131.57,131.09,131.08,127.52,81.53,28.26$.

ESI MS found for $\left[\mathrm{M}^{+\cdot}\right] \mathrm{m} / \mathrm{z}$ 256.0106; calculated for $\left[\mathrm{M}^{++}\right] \mathrm{m} / \mathrm{z} 256.0099$.

\section{Tert-butyl 4-(4,4,5,5-tetramethyl-1,3,2-dioxaborolan-2-yl)benzoate (1c).}

To a solution of tert-butyl 4-bromobenzoate (1b) $(10.0 \mathrm{~g}, 39 \mathrm{mmol})$ and Bis(pinacolato)diboron (13.9 $\mathrm{g}$, $55 \mathrm{mmol})$ in dioxane $(150 \mathrm{~mL})$ was added KOAc $(15.0 \mathrm{~g}, 155 \mathrm{mmol})$. The solution was degassed by 
sparging with Ar for 30 mins. To the degassed solution was added [1,1'-bis(diphenylphosphino)ferrocene]dichloropalladium(II) $(1.0 \mathrm{~g}, 1.4 \mathrm{mmol})$ under a constent stream of Ar. The solution was refluxed at $100^{\circ} \mathrm{C}$ for overnight. The solvent was then removed in vacuo. The crude productwas taken up in EtOAc and washed with water $(3 \times 250 \mathrm{~mL})$. The separated organic layer was dried over $\mathrm{MgSO}_{4}$, filtered, concentrated in vacuo, and further purified by column chromatography (hexanes/ethyl acetate v/v 20:1). The pure product was dried in vacuo to give an off white solid. Yield: $7.9 \mathrm{~g}, 67 \%$.

${ }^{1} \mathrm{HNMR}\left(300 \mathrm{MHz}, \mathrm{CDCl}_{3}\right) \delta 7.99(\mathrm{~d}, J=8.1 \mathrm{~Hz}, 2 \mathrm{H}), 7.87(\mathrm{~d}, J=8.4 \mathrm{~Hz}, 2 \mathrm{H}), 1.62(\mathrm{~s}, 9 \mathrm{H}), 1.37(\mathrm{~s}, 12 \mathrm{H})$.

${ }^{13} \mathrm{C} \mathrm{NMR}\left(75 \mathrm{MHz}, \mathrm{CDCl}_{3}\right) \delta 165.81,134.52,134.24,128.42,84.10,81.10,28.19,24.88$.

ESI MS found for $\left[\mathrm{M}^{++}\right] \mathrm{m} / \mathrm{z}$ 304.1846; calculated for $\left[\mathrm{M}^{++}\right] \mathrm{m} / \mathrm{z} 304.1846$.

\section{(4-(Tert-butoxycarbonyl)phenyl)boronic acid (1d).}

To a suspension of tert-butyl 4-(4,4,5,5-tetramethyl-1,3,2-dioxaborolan-2-yl)benzoate (1c) (7.2 g, 23.6 mmol)stirring at room temperature in an acetone/water mixture $(280 \mathrm{~mL}, \mathrm{v} / \mathrm{v} 1: 1)$ was added $\mathrm{NH}_{4} \mathrm{OAc}$ $(4.2 \mathrm{~g}, 54.4 \mathrm{mmol})$ and $\mathrm{NaIO}_{4}(11.6 \mathrm{~g}, 54.4 \mathrm{mmol})$. The susupension was allowed to continue stirring at room temperature overnight. The solvent was then removed in vacuo. The crude product was taken up in EtOAc $(200 \mathrm{~mL})$ and washed with brine $(2 \times 100 \mathrm{~mL})$ and water $(1 \times 100 \mathrm{~mL})$. The organic layer was then separated and dried over $\mathrm{MgSO}_{4}$ and further dried invacuo to give the desired product as a white solid. Yield: $83 \%, 4.36 \mathrm{~g}$.

${ }^{1}$ HNMR (300 MHz, DMSO-d $\left.d_{6}\right) \delta 8.29(\mathrm{~s}, 2 \mathrm{H}), 7.92(\mathrm{~d}, J=8.4 \mathrm{~Hz}, 2 \mathrm{H}), 7.79(\mathrm{~d}, J=8.1 \mathrm{~Hz}, 2 \mathrm{H}), 1.55(\mathrm{~s}, 9 \mathrm{H})$.

ESI MS found for $\left[\mathrm{M}+\mathrm{Na}^{+}\right] \mathrm{m} / \mathrm{z} 245.0968$; calculated for $\left[\mathrm{M}^{++}\right] \mathrm{m} / \mathrm{z} 245.0961$.

\section{Di-tert-butyl 5'-formyl-[1,1':3',1"-terphenyl]-4,4"-dicarboxylate (1f).}

A solution of (4-(tert-butoxycarbonyl)phenyl)boronic acid(1d) $(4.0 \mathrm{~g}, 18.0 \mathrm{mmol}), 3,5$ dibromobenzaldehyde (1e) $(2.05 \mathrm{~g}, 7.8 \mathrm{mmol})$, and potassium carbonate $(2.76 \mathrm{~g}, 19.9 \mathrm{mmol})$ in a water/dioxane mixture $(60 \mathrm{~mL}, \mathrm{v} / \mathrm{v} 1: 4)$ was degassed with Ar bubbling for 30 minutes. $\mathrm{Pd}_{(}\left(\mathrm{PPh}_{3}\right)_{4}(0.39 \mathrm{~g}$, $0.3 \mathrm{mmol}$ ) was added and the solution was refluxed under an Ar atomosphere for 18 hours at $100^{\circ} \mathrm{C}$. The reaction was then cooled to room temperature, poured onto water $(100 \mathrm{~mL})$, and extracted with ethyl acetate $(3 \times 50 \mathrm{~mL})$. The combined organic layer was dried over $\mathrm{MgSO}_{4}$, filtered, concentrated, and purified by column chromatography with an elution of hexanes/ethyl acetate ( $v / v 5: 1)$. The desired product was dried in vacuo to give an off white solid. Yield: $\mathrm{g}, 73 \% 2.60 \mathrm{~g}$.

${ }^{1} \mathrm{HNMR}\left(300 \mathrm{MHz}, \mathrm{CDCl}_{3}\right) \delta 10.17(\mathrm{~s}, 1 \mathrm{H}), 8.14-8.10(\mathrm{~m}, 6 \mathrm{H})$, contained in this multiplet: $8.12(\mathrm{~d}, J=1.8$ $\mathrm{Hz}, 2 \mathrm{H})$ and $8.11(\mathrm{~d}, J=8.0 \mathrm{~Hz}, 4 \mathrm{H}), 8.08(\mathrm{t}, J=1.8 \mathrm{~Hz}, 1 \mathrm{H}), 7.72(\mathrm{~d}, J=8.5 \mathrm{~Hz}, 4 \mathrm{H}), 1.63(\mathrm{~s}, 18 \mathrm{H})$. 
${ }^{13} \mathrm{CNMR}\left(75 \mathrm{MHz}, \mathrm{CDCl}_{3}\right) \delta 191.95,165.49,143.44,142.10,137.73,131.94,131.90,130.34,127.93$, $127.17,81.44,28.34$.

ESI MS found for $\left[\mathrm{M}^{+\cdot}\right] \mathrm{m} / \mathrm{z}$ 458.2109; calculated for $\left[\mathrm{M}^{+\cdot}\right] \mathrm{m} / \mathrm{z} 458.2093$.

\section{Tetrakis 3,5-bis[(4-tert-butoxycarbonyl)phenyl]phenyl porphine (1g).}

To a degassed solution of di-tert-butyl 5'-formyl-[1,1':3',1"-terphenyl]-4,4"-dicarboxylate (1f) (2.29 g, 5.0 $\mathrm{mmol})$ and pyrrole $(347 \mu \mathrm{L}, 5.0 \mathrm{mmol})$ in $\mathrm{CH}_{2} \mathrm{Cl}_{2}(500 \mathrm{~mL})$ was added $\mathrm{BF}_{3} \cdot \mathrm{Et}_{2} \mathrm{O}(80 \mu \mathrm{L}, 0.6 \mathrm{mmol})$ via syringe. The solution was shaded from light and stirred under Ar for 3 hours. Next,2,3-Dichloro-5,6dicyano- $p$-benzoquinone $(0.8526 \mathrm{~g}, 3.8 \mathrm{mmol})$ was added portion-wise and the reaction was allowed to continue stirring under Ar for 8 hours. The reaction mixture was concentrated in vacuo and purified by column chromatography $\left(\mathrm{CHCl}_{3}\right)$. The red fluorescent band was collected, dried in vacuo, and further suspended in $\mathrm{MeOH}(200 \mathrm{~mL})$. The pure product was collected as a lustrous purple solid by filtration. Yield: 22\%, $556 \mathrm{mg}$.

${ }^{1} \mathrm{HNMR}\left(300 \mathrm{MHz}, \mathrm{CDCl}_{3}\right) \delta 9.09(\mathrm{~s}, 8 \mathrm{H}), 8.58(\mathrm{~d}, J=1.5 \mathrm{~Hz}, 8 \mathrm{H}), 8.33(\mathrm{t}, J=1.5 \mathrm{~Hz}, 4 \mathrm{H}), 8.16(\mathrm{~d}, J=8.4 \mathrm{~Hz}$, $16 \mathrm{H}), 7.98(\mathrm{~d}, \mathrm{~J}=8.4 \mathrm{~Hz}, 16 \mathrm{H}), 1.64(\mathrm{~s}, 72 \mathrm{H}),-2.59(\mathrm{~s}, 2 \mathrm{H})$.

${ }^{13} \mathrm{CNMR}\left(75 \mathrm{MHz}, \mathrm{CDCl}_{3}\right) \delta 165.67,144.60,143.50,139.57,133.30,131.57,130.35,127.52,125.84$, $119.92,81.34,28.36$.

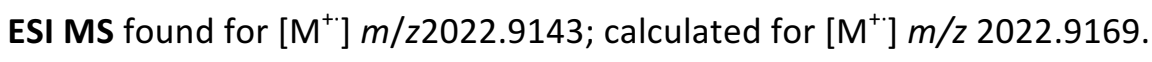


S2.2 Synthesis and Characterization of $\mathrm{Mn}^{\mathrm{II \prime}}$ and $\mathrm{Fe}^{\mathrm{III}}$ metallated porphyrin ligands 2a-d.

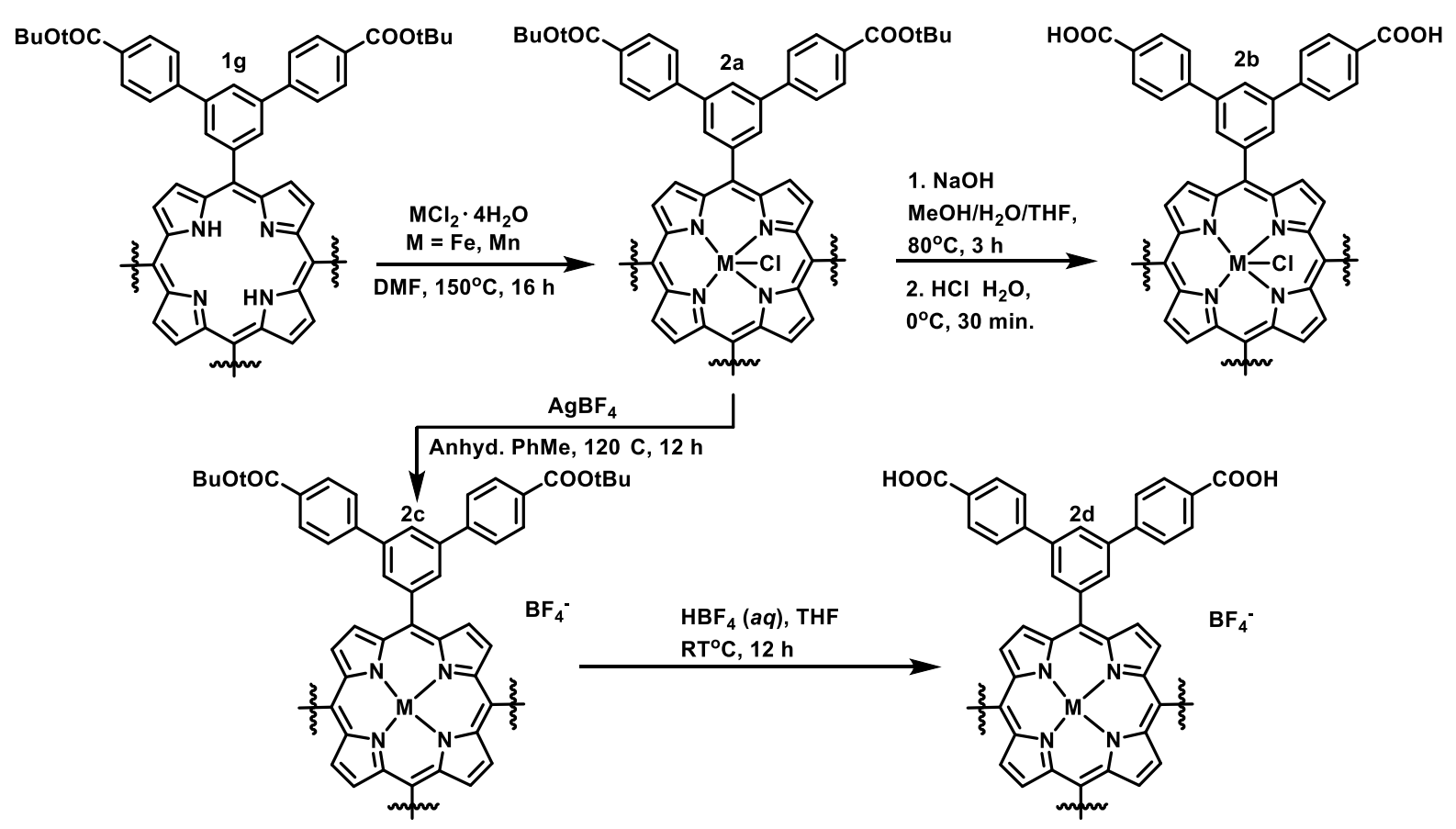

Scheme S2. Synthesis of $\mathrm{Mn}^{\prime \prime \prime}$ and Fe"' metallated porphyrin ligands 2a-d.

\section{Manganese (III) tetrakis 3,5-bis[(4-tert-butoxycarbonyl)phenyl]phenyl porphine chloride (2a-Mn).}

A mixture of tetrakis 3,5-bis[(4-tert-butoxycarbonyl)phenyl]phenyl porphine $(1 \mathrm{~g})(400 \mathrm{mg}, 0.20 \mathrm{mmol})$ and $\mathrm{MnCl}_{2} \cdot 4 \mathrm{H}_{2} \mathrm{O}(198 \mathrm{mg}, 1.0 \mathrm{mmol})$ in DMF $(60 \mathrm{~mL})$ was stirred at $110^{\circ} \mathrm{C}$ for 6 hours. The mixture was cooled to room temperature and poured onto water $(300 \mathrm{~mL})$. The mixture was further cooled to $-20^{\circ} \mathrm{C}$, filtered, and washed with water. The product was then dried in vacuo at $100^{\circ} \mathrm{C}$ for 16 hours to give the target compound as a green solid.Yield:76\%, $321 \mathrm{mg}$.

UV-Vis (PhMe) $\lambda_{\max }: 479.9 \mathrm{~nm}$ (Soret band), $583.8 \mathrm{~nm}, 619.1 \mathrm{~nm}$ (Q-bands).

ESI MS found for[M-Cl'] m/z 2076.8315; calculated for $\left[\mathrm{M}^{++}\right] \mathrm{m} / \mathrm{z} 2076.8426$.

\section{Mangenese (III) tetrakis 3,5-bis[4-carboxyphenyl]phenyl porphine chloride (2b-Mn).}

To a solution ofmanganese (III) tetrakis 3,5-bis[(4-tert-butoxycarbonyl)phenyl]phenyl porphine chloride $(2 \mathrm{a}-\mathrm{Mn})(160 \mathrm{mg}, 0.08 \mathrm{mmol})$ in a mixture of THF $(20 \mathrm{~mL})$ and methanol $(10 \mathrm{~mL})$ was added a solution of $\mathrm{KOH}$ in water $(0.1 \mathrm{M}, 20 \mathrm{~mL})$. The mixture was refluxed at $80^{\circ} \mathrm{C}$ for $3 \mathrm{hrs}$. The organic solvents wereremoved in vacuo and the aqueous solution was cooled to $0^{\circ} \mathrm{C}$. The solution was acidified to a pH of 1 with $1 \mathrm{M} \mathrm{HCl}$. The resultant dark green precipitate was collected by vacuum filtration, washed with water, and dried in a vacuum oven over night at $100^{\circ} \mathrm{C}$.Yield: $99 \%, 125 \mathrm{mg}$.

UV-Vis (DMF) $\lambda_{\max }: 474.0 \mathrm{~nm}$ (Soret band), $579.5 \mathrm{~nm}, 615.0 \mathrm{~nm}$ (Q-bands). 


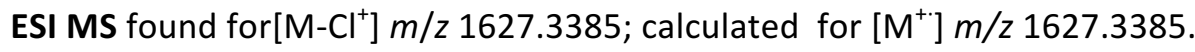

Manganese (III) tetrakis 3,5-bis[(4-tert-butoxycarbonyl)phenyl]phenyl porphine tetrafluoroborate (2cMn).

A mixture of manganese (III) tetrakis 3,5-bis[(4-tert-butoxycarbonyl)phenyl]phenyl porphine chloride(2a$\mathrm{Mn})(160 \mathrm{mg}, 0.08 \mathrm{mmol})$ andAgBF $4(15 \mathrm{mg}, 0.08 \mathrm{mmol})$ in dry $\mathrm{CH}_{2} \mathrm{Cl}_{2}(30.0 \mathrm{~mL})$ was stirred at room temperature for 3 hours. The solution was then filtered and concentrated in vacuo to give the target compound as a green solid. Yield:95\%, $156 \mathrm{mg}$.

UV-Vis (PhMe) $\lambda_{\max }: 483.6 \mathrm{~nm}$ (Soret band), $570.5 \mathrm{~nm}, 604.6 \mathrm{~nm}$ (Q-bands).

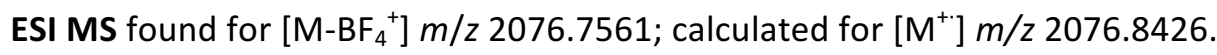

Mangenese (III) tetrakis 3,5-bis[4-carboxyphenyl]phenyl porphine tetrafluoroborate (2d-Mn).

To a solution of manganese (III) tetrakis 3,5-bis[(4-tert-butoxycarbonyl)phenyl]phenyl porphinetetrafluoroborate $(2 \mathrm{c}-\mathrm{Mn})(150 \mathrm{mg})$ in THF $(30 \mathrm{~mL})$ at $0^{\circ} \mathrm{C}$, was added portionwisea solution of $\mathrm{HBF}_{4}$ in water (48 wt\%, $50 \mathrm{~mL}$ ). The solution was allowed to warm to room temperature and was stirred for 12 hours. The resultant green precipitate was collected by vacuum filtration, washed thoroughly with water, and then dried in a vacuum oven over night at $100^{\circ} \mathrm{C}$. Yield: $97 \%, 123 \mathrm{mg}$.

UV-Vis (DMF) $\lambda_{\max }: 471.0 \mathrm{~nm}$ (Soret band), $574.5 \mathrm{~nm}, 610.0 \mathrm{~nm}$ (Q-bands).

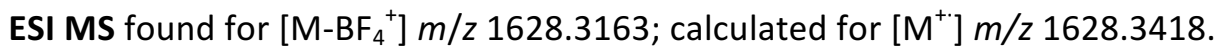

Iron (III) tetrakis 3,5-bis[(4-tert-butoxycarbonyl)phenyl]phenyl porphine chloride (2a-Fe).

A mixture of tetrakis 3,5-bis[(4-tert-butoxycarbonyl)phenyl]phenyl porphine (1g) (400mg, $0.2 \mathrm{mmol}$ ) and $\mathrm{FeCl}_{2} \cdot 4 \mathrm{H}_{2} \mathrm{O}(199 \mathrm{mg}, 1.0 \mathrm{mmol})$ in DMF $(60 \mathrm{~mL})$ was stirred at $110^{\circ} \mathrm{C}$ for 6 hours. The mixture was cooled to room temperature and poured onto water $(300 \mathrm{~mL})$. The mixture was further cooled to $-20^{\circ} \mathrm{C}$ and filtered. The resultant solid was then dissolved in $\mathrm{CHCl}_{3}$ and washed with $1 \mathrm{M} \mathrm{HCl}(1 \times 100 \mathrm{~mL})$ and water $(2 \times 100 \mathrm{~mL})$ The product was then dried in vacuo at $100^{\circ} \mathrm{C}$ for 16 hours to give the target compound as a reddish brown solid.Yield: $65 \%, 275 \mathrm{mg}$.

UV-Vis (PhMe) $\lambda_{\text {max: }}: 400.1 \mathrm{~nm}$ (Soret band), $520.2 \mathrm{~nm}$ (Q-band).

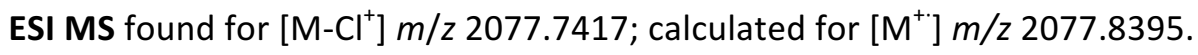

Iron (III) tetrakis 3,5-bis[4-carboxyphenyl]phenyl porphine chloride (2b-Fe).

To a solution of iron (III) tetrakis 3,5-bis[(4-tert-butoxycarbonyl)phenyl]phenyl porphine chloride (2a-Fe) $(135 \mathrm{mg}, 0.06 \mathrm{mmol})$ in a mixture of THF $(20 \mathrm{~mL})$ and methanol $(10 \mathrm{~mL})$ was added a solution of $\mathrm{KOH}$ in water $(0.1 \mathrm{M}, 20 \mathrm{~mL})$. The mixture was refluxed at $80^{\circ} \mathrm{C}$ for $3 \mathrm{hrs}$. The organic solvents wereremoved in vacuo and the aqueous solution was cooled to $0^{\circ} \mathrm{C}$. The solution was acidified to a pH of 1 with $1 \mathrm{M} \mathrm{HCl}$. The resultant dark reddish brown precipitate was collected by vacuum filtration, washed with water, and dried in a vacuum oven over night at $100^{\circ} \mathrm{C}$. Yield: $96 \%, 102 \mathrm{mg}$. 
UV-Vis (DMF) $\lambda_{\text {max }}: 422.7 \mathrm{~nm}$ (Soret band), $513.4 \mathrm{~nm}$ (Q-band).

Iron (III) tetrakis 3,5-bis[(4-tert-butoxycarbonyl)phenyl]phenyl porphinetetrafluoroborate (2c-Fe).

A mixture iron (III) tetrakis 3,5-bis[(4-tert-butoxycarbonyl)phenyl]phenyl porphine chloride(2a-Fe) (135 $\mathrm{mg}, 0.06 \mathrm{mmol})$ and $\mathrm{AgBF}_{4}(11 \mathrm{mg}, 0.06 \mathrm{mmol})$ in dry $\mathrm{CH}_{2} \mathrm{Cl}_{2}(30.0 \mathrm{~mL})$ was stirred at room temperature for 3 hours. The solution was then filtered and concentrated in vacuo to give the target compound as a reddish brown solid. Yield: 95\%, $131 \mathrm{mg}$.

UV-Vis (PhMe) $\lambda_{\text {max }}: 414.9 \mathrm{~nm}$ (Soret band), $508.9 \mathrm{~nm}$ (Q-band).

Iron (III) tetrakis 3,5-bis[4-carboxyphenyl]phenyl porphine tetrafluoroborate (2d-Fe).

To a solution of iron (III) tetrakis 3,5-bis[(4-tert-butoxycarbonyl)phenyl]phenyl porphinetetrafluoroborate (2c-Fe) $(130 \mathrm{mg})$ in THF $(30 \mathrm{~mL})$ at $0^{\circ} \mathrm{C}$, was added portionwise a solution of $\mathrm{HBF}_{4}$ in water $(48 \mathrm{wt} \%, 50$ $\mathrm{mL}$ ). The solution was allowed to warm to room temperature and was stirred for 12 hours. The resultant reddish brown precipitate was collected by vacuum filtration, washed thoroughly with water, and then dried in a vacuum oven over night at $100^{\circ} \mathrm{C}$. Yield: $96 \%, 106 \mathrm{mg}$.

UV-Vis (DMF) $\lambda_{\max }: 420.9 \mathrm{~nm}$ (Soret band), $512.9 \mathrm{~nm}$ (Q-band). 
a)
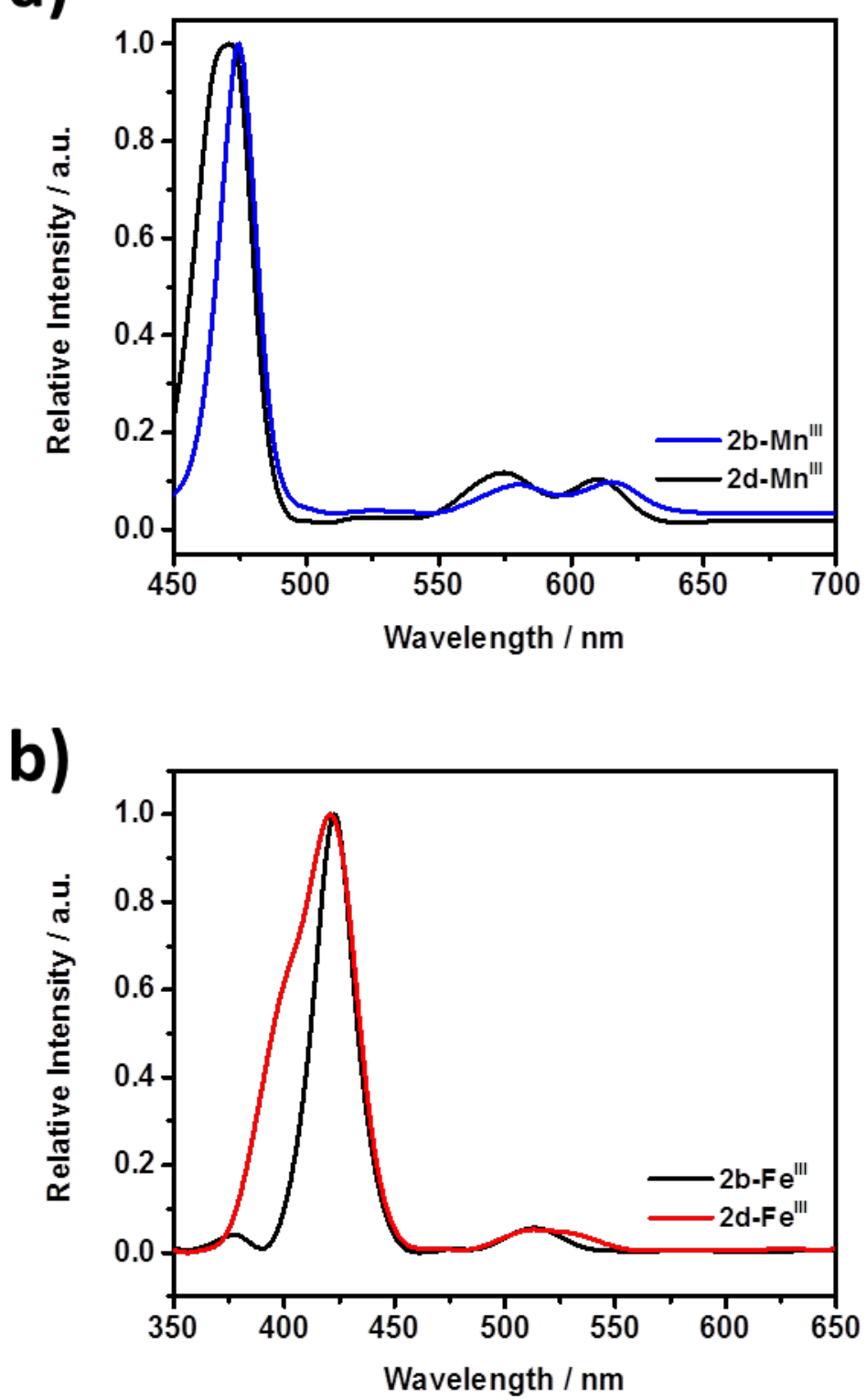

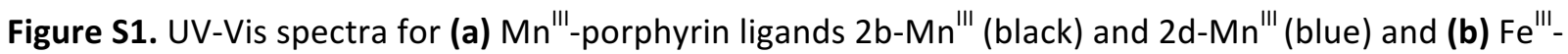
porphyrin ligands $2 \mathrm{~b}-\mathrm{Fe}^{\text {III }}$ (black) and $2 \mathrm{~d}-\mathrm{Fe}^{\mathrm{III}}$ (red). 


\section{UNLPF-13.}

A solution of $\mathbf{2 b}-\mathrm{Mn}(10 \mathrm{mg}, 6.0 \mu \mathrm{mol})$ and $\ln \left(\mathrm{NO}_{3}\right)_{3} \cdot \mathrm{H}_{2} \mathrm{O}(5.4 \mathrm{mg}, 18 \mu \mathrm{mol})$ in a mixture of $\mathrm{N}, \mathrm{N}^{\prime}-$ dimethylformamide (DMF) $(5 \mathrm{~mL})$ and acetic acid $(0.1 \mathrm{~mL})$ was sonicated for 1 hour.The solution was then heated at $80^{\circ} \mathrm{C}$ for 72 hours followed by cooling to room temperature over a period of 4 hours. The resulting black octahedral crystals were washed with fresh DMF and $\mathrm{MeOH}$ and collected by vacuum filtration to give pure UNLPF-13.

\section{UNLPF-14.}

A solution of $2 \mathrm{~d}-\mathrm{Mn}(10 \mathrm{mg}, 6.0 \mu \mathrm{mol})$ and $\ln \left(\mathrm{NO}_{3}\right)_{3} \cdot \mathrm{H}_{2} \mathrm{O}(5.4 \mathrm{mg}, 18 \mu \mathrm{mol})$ in a mixture of $\mathrm{N}, \mathrm{N}^{\prime}-$ dimethylformamide (DMF) $(5 \mathrm{~mL})$ and acetic acid $(0.1 \mathrm{~mL})$ was sonicated for 1 hour. The solution was then heated at $80^{\circ} \mathrm{C}$ for 72 hours followed by cooling to room temperature over a period of 4 hours. The resulting black octahedral crystals were washed with fresh $\mathrm{DMF}$ and $\mathrm{MeOH}$ and collected by vacuum filtration to give pure UNLPF-14.

\section{UNLPF-15.}

A solution of $2 \mathbf{b}-\mathrm{Fe}(10 \mathrm{mg}, 6.0 \mu \mathrm{mol})$ and $\ln \left(\mathrm{NO}_{3}\right)_{3} \cdot \mathrm{H}_{2} \mathrm{O}(5.4 \mathrm{mg}, 18 \mu \mathrm{mol})$ in a mixture of $\mathrm{N}, \mathrm{N}^{\prime}-$ dimethylformamide (DMF) $(5 \mathrm{~mL})$ and acetic acid $(0.1 \mathrm{~mL})$ was sonicated for 1 hour. The solution was then heated at $80^{\circ} \mathrm{C}$ for 72 hours followed by cooling to room temperature over a period of 4 hours. The resulting black octahedral crystals were washed with fresh $\mathrm{DMF}$ and $\mathrm{MeOH}$ and collected by vacuum filtration to give pure UNLPF-15.

\section{UNLPF-16.}

A solution of $2 \mathrm{~d}-\mathrm{Fe}(10 \mathrm{mg}, 6.0 \mu \mathrm{mol})$ and $\ln \left(\mathrm{NO}_{3}\right)_{3} \cdot \mathrm{H}_{2} \mathrm{O}(5.4 \mathrm{mg}, 18 \mu \mathrm{mol})$ in a mixture of $\mathrm{N}, \mathrm{N}^{\prime}$ dimethylformamide (DMF) $(5 \mathrm{~mL})$ and acetic acid $(0.1 \mathrm{~mL})$ was sonicated for 1 hour. The solution was then heated at $80^{\circ} \mathrm{C}$ for 72 hours followed by cooling to room temperature over a period of 4 hours. The resulting black octahedral crystals were washed with fresh DMF and $\mathrm{MeOH}$ and collected by vacuum filtration to give pure UNLPF-16. 


\section{S2.3 Crystallographic Data for UNLPF-13, -14, -15, and -16}

Single crystal $X$-ray diffraction data was collected using synchrotron radiation, $\lambda=0.41328 \AA$, at the Advanced Photon Source, Chicago, IL. Indexing was performed using APEX2 (Difference Vectors method). ${ }^{1}$ Data integration and reduction were performed using SaintPlus $6.01 .{ }^{2}$ Absorption correction was performed by multi-scan method implemented in SADABS. ${ }^{3}$ Space groups were determined using XPREP implemented in APEX2. ${ }^{1}$ The structures were solved using the program XT and refined using SHELXL-2013 (full-matrix least-squares on $F^{2}$ ) contained in APEX2 and WinGX v1.70.00. $.^{4-7} \mathrm{C}, \mathrm{N}, \mathrm{O}$ atoms were refined with anisotropic displacement parameters and $\mathrm{H}$ atoms were placed in geometrically calculated positions and included in the refinement process using riding model with isotropic thermal parameters: $U_{\text {iso }}(H)=1.2 U_{\text {eq }}(-\mathrm{CH})$. The contribution of disordered solvent molecules was treated as diffuse using the SQUEEZE procedure implemented in PLATON. ${ }^{8}$ Crystal data and refinement conditions are shown in Tables S1-S4. CCDC 1042957 and 1451927-1451929 contains the supplementary crystallographic data for this UNLPF-13, $-14,-15$, and -16 . This data can be obtained free of charge from The Cambridge Crystallographic Data Centre via www.ccdc.cam.ac.uk/data_request/cif.

Table S1. Crystal data and structure refinement for UNLPF-13

\begin{tabular}{|c|c|}
\hline Identification & UNLPF-13 \\
\hline empirical formula & $\mathrm{C}_{100} \mathrm{H}_{52} \mathrm{ClIn}_{2} \mathrm{MnN}_{4} \mathrm{O}_{16}$ \\
\hline formula weight $\left(\mathrm{g} \cdot \mathrm{mol}^{-1}\right)$ & 1885.49 \\
\hline temperature $(\mathrm{K})$ & $100(2) \mathrm{K}$ \\
\hline wavelength $(\AA)$ & 0.47398 \\
\hline crystal system, space group & orthorhombic, Pnnm \\
\hline$a(\AA)$ & $23.767(10)$ \\
\hline$b(\AA)$ & 23.767 \\
\hline$c(\AA)$ & $29.459(12)$ \\
\hline$\alpha$ (deg) & 90.00 \\
\hline$\beta$ (deg) & 90.00 \\
\hline$\gamma(\operatorname{deg})$ & 90.00 \\
\hline volume $\left(\AA^{3}\right)$ & $16641(9)$ \\
\hline$Z$, density (calcd) $\left(\mathrm{Mg} \cdot \mathrm{m}^{-3}\right)$ & $2,0.376$ \\
\hline abs coefficient $\left(\mathrm{mm}^{-1}\right)$ & 0.200 \\
\hline$F(000)$ & 1896 \\
\hline crystal size $\left(\mathrm{mm}^{3}\right)$ & $0.2 \times 0.2 \times 0.2 \mathrm{~mm}$ \\
\hline$\theta$ range (deg) & 0.74 to $9.97 \mathrm{deg}$ \\
\hline reflections collected/unique & 3449 / 1782 \\
\hline completeness to $\theta(\%)$ & $97.8 \%$ \\
\hline refinement method & Full-matrix least-squares on $F^{2}$ \\
\hline data / restraints / parameters & 3449 / 289 / 233 \\
\hline goodness-of-fit on $F^{2}$ & 0.894 \\
\hline final Rindices [I>2sigma(I)] & $R_{1}=0.0579, w R_{2}=0.1560$ \\
\hline largest diff. peak and hole (e. $\mathrm{A}^{-3}$ ) & 0.29 and -0.19 \\
\hline
\end{tabular}


Table S2. Crystal data and structure refinement for UNLPF-14

\begin{tabular}{|c|c|}
\hline Identification & UNLPF-14 \\
\hline empirical formula & $\mathrm{C}_{100} \mathrm{H}_{54} \mathrm{In}_{2} \mathrm{MnN}_{4} \mathrm{O}_{17}$ \\
\hline formula weight $\left(\mathrm{g} \cdot \mathrm{mol}^{-1}\right)$ & 1868.08 \\
\hline temperature $(\mathrm{K})$ & $296(2) \mathrm{K}$ \\
\hline wavelength $(\AA)$ & 0.41328 \\
\hline crystal system, space group & orthorhombic, Pnnm \\
\hline$a(\AA)$ & $23.4939(13)$ \\
\hline$b(\AA)$ & $23.4954(14)$ \\
\hline$c(\AA)$ & $31.9724(18)$ \\
\hline$\alpha$ (deg) & 90.00 \\
\hline$\beta$ (deg) & 90.00 \\
\hline$\gamma(\operatorname{deg})$ & 90.00 \\
\hline volume $\left(\AA^{3}\right)$ & $17648.7(17)$ \\
\hline$Z$, density (calcd) $\left(\mathrm{Mg} \cdot \mathrm{m}^{-3}\right)$ & $2,0.351$ \\
\hline abs coefficient $\left(\mathrm{mm}^{-1}\right)$ & 0.187 \\
\hline$F(000)$ & 1878 \\
\hline crystal size $\left(\mathrm{mm}^{3}\right)$ & $0.2 \times 0.2 \times 0.2 \mathrm{~mm}$ \\
\hline$\theta$ range (deg) & 0.80 to $14.16 \mathrm{deg}$ \\
\hline reflections collected/unique & 15379 / 7316 \\
\hline completeness to $\theta(\%)$ & $98.3 \%$ \\
\hline refinement method & Full-matrix least-squares on $F^{2}$ \\
\hline data / restraints / parameters & 15379 / 293 / 2 \\
\hline goodness-of-fit on $F^{2}$ & 0.946 \\
\hline final Rindices [I>2sigma(I)] & $R_{1}=0.0832, w R_{2}=0.2593$ \\
\hline largest diff. peak and hole (e. $\mathrm{A}^{-3}$ ) & 0.79 and -0.49 \\
\hline
\end{tabular}


Table S3. Crystal data and structure refinement for UNLPF-15

\begin{tabular}{|c|c|}
\hline Identification & UNLPF-15 \\
\hline empirical formula & $\mathrm{C}_{100} \mathrm{H}_{52} \mathrm{ClFeln}_{2} \mathrm{~N}_{4} \mathrm{O}_{16}$ \\
\hline formula weight $\left(\mathrm{g} \cdot \mathrm{mol}^{-1}\right)$ & 1886.40 \\
\hline temperature $(\mathrm{K})$ & $296(2) \mathrm{K}$ \\
\hline wavelength $(\AA)$ & 0.47398 \\
\hline crystal system, space group & orthorhombic, Pnnm \\
\hline$a(\AA)$ & $23.412(6)$ \\
\hline$b(\AA)$ & $23.428(6)$ \\
\hline$c(\AA)$ & $32.108(9)$ \\
\hline$\alpha$ (deg) & 90.00 \\
\hline$\beta$ (deg) & 90.00 \\
\hline$\gamma(\operatorname{deg})$ & 90.00 \\
\hline volume $\left(\AA^{3}\right)$ & $17611(8)$ \\
\hline$Z$, density (calcd) $\left(\mathrm{Mg} \cdot \mathrm{m}^{-3}\right)$ & $2,0.356$ \\
\hline abs coefficient $\left(\mathrm{mm}^{-1}\right)$ & 0.067 \\
\hline$F(000)$ & 1898.0 \\
\hline crystal size $\left(\mathrm{mm}^{3}\right)$ & $0.2 \times 0.2 \times 0.2 \mathrm{~mm}$ \\
\hline$\theta$ range (deg) & 0.72 to $13.12 \mathrm{deg}$ \\
\hline reflections collected/unique & 8212 / 3421 \\
\hline completeness to $\theta(\%)$ & $98.8 \%$ \\
\hline refinement method & Full-matrix least-squares on $F^{2}$ \\
\hline data / restraints / parameters & 8212 / 289 / 196 \\
\hline goodness-of-fit on $F^{2}$ & 0.930 \\
\hline final Rindices [I>2sigma(I)] & $R_{1}=0.0803, w R_{2}=0.2552$ \\
\hline largest diff. peak and hole (e. $\mathrm{A}^{-3}$ ) & 0.46 and -0.69 \\
\hline
\end{tabular}


Table S4. Crystal data and structure refinement for UNLPF-16

\begin{tabular}{|c|c|}
\hline Identification & UNLPF-16 \\
\hline empirical formula & $\mathrm{C}_{100} \mathrm{H}_{54} \mathrm{Feln}_{2} \mathrm{~N}_{4} \mathrm{O}_{17}$ \\
\hline formula weight $\left(\mathrm{g} \cdot \mathrm{mol}^{-1}\right)$ & 1866.95 \\
\hline temperature $(\mathrm{K})$ & $296(2) \mathrm{K}$ \\
\hline wavelength $(\AA)$ & 0.41328 \\
\hline crystal system, space group & orthorhombic, Pnnm \\
\hline$a(\AA)$ & $23.4897(13)$ \\
\hline$b(\AA)$ & $23.4895(14)$ \\
\hline$c(\AA)$ & $31.9645(18)$ \\
\hline$\alpha$ (deg) & 90.00 \\
\hline$\beta$ (deg) & 90.00 \\
\hline$\gamma(\operatorname{deg})$ & 90.00 \\
\hline volume $\left(\AA^{3}\right)$ & $17636.8(17)$ \\
\hline$Z$, density (calcd) $\left(\mathrm{Mg} \cdot \mathrm{m}^{-3}\right)$ & $2,0.353$ \\
\hline abs coefficient $\left(\mathrm{mm}^{-1}\right)$ & 0.101 \\
\hline$F(000)$ & 1880 \\
\hline crystal size $\left(\mathrm{mm}^{3}\right)$ & $0.2 \times 0.2 \times 0.2 \mathrm{~mm}$ \\
\hline$\theta$ range $(\mathrm{deg})$ & 0.63 to $14.58 \mathrm{deg}$ \\
\hline reflections collected/unique & 16843 / 7581 \\
\hline completeness to $\theta(\%)$ & $98.9 \%$ \\
\hline refinement method & Full-matrix least-squares on $F^{2}$ \\
\hline data / restraints / parameters & $16843 / 176 / 289$ \\
\hline goodness-of-fit on $F^{2}$ & 0.991 \\
\hline final Rindices [I>2sigma(I)] & $R_{1}=0.0963, w R_{2}=0.3282$ \\
\hline largest diff. peak and hole (e. $\mathrm{A}^{-3}$ ) & 0.70 and -0.92 \\
\hline
\end{tabular}


a)

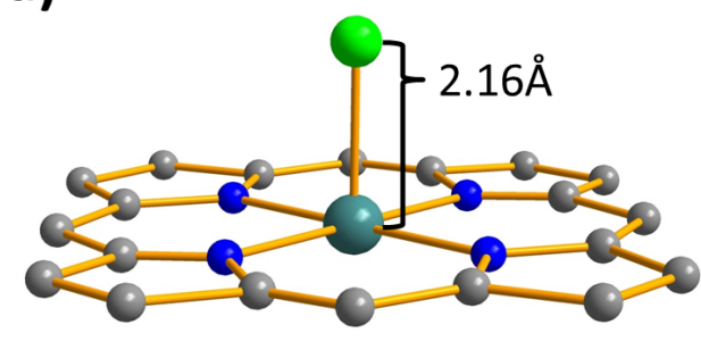

c)

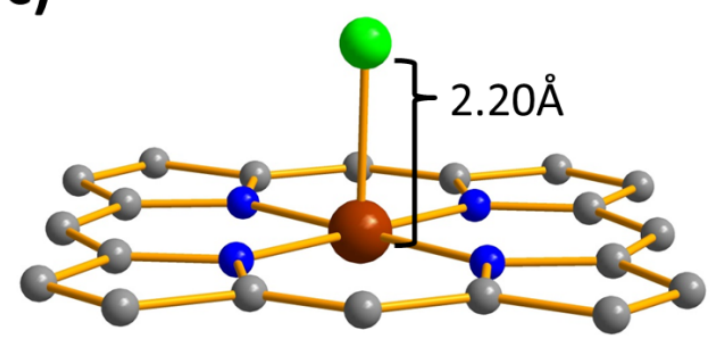

b)

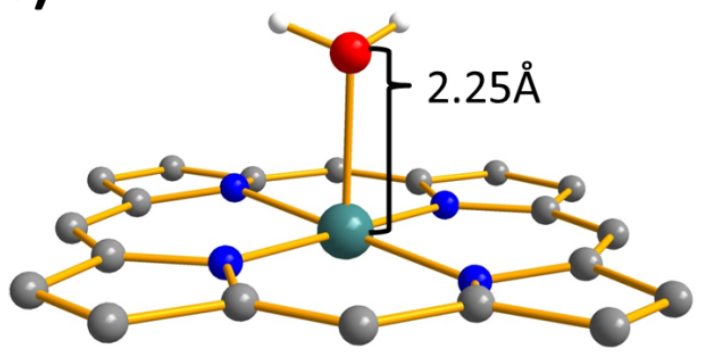

d)

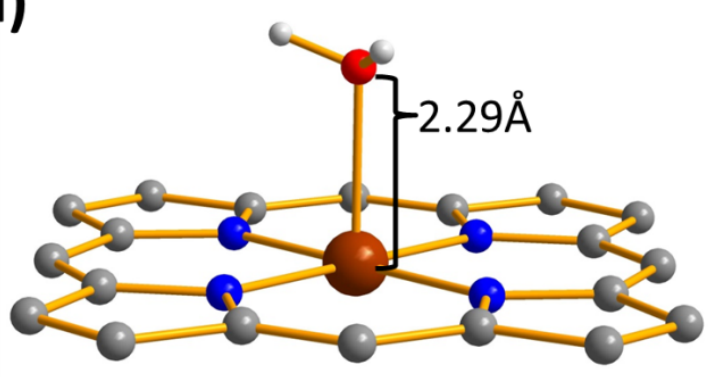

Figure S2. Axial ligand distances for UNLPF-13 (a), -14 (b), -15 (c), -16 (d). 


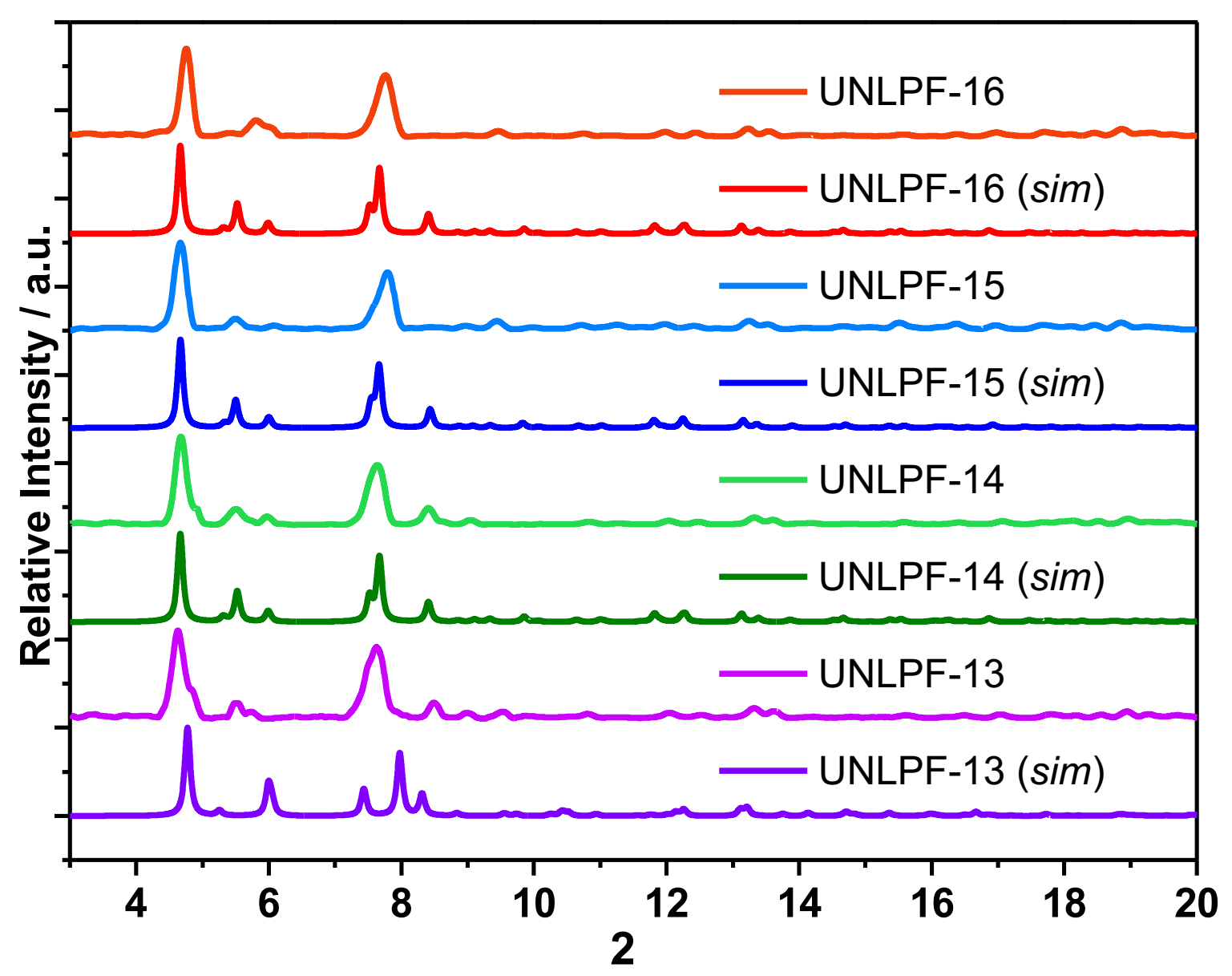

Figure S3. Simulated and experimental powder X-ray diffraction patterns of UNLPF-13 (purple), UNLPF14 (green), UNLPF-15 (blue), and UNLPF-16 (red). 


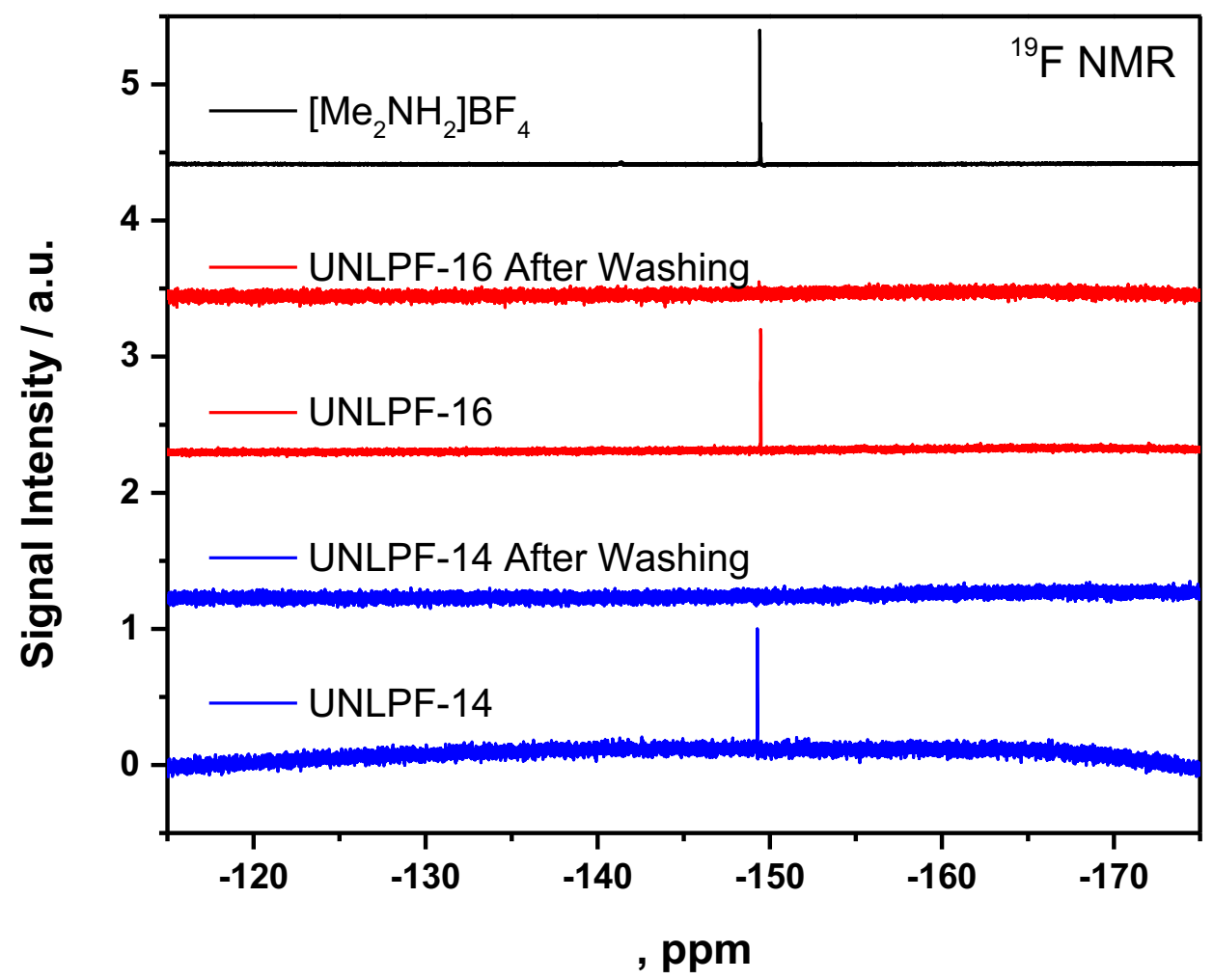

Figure S4. ${ }^{19} \mathrm{~F}$ NMR of acid digested samples of UNLPF-14 (blue) and UNLPF-16 (red) before and after washing fresh DMF (exchanged $3 x$ over 72 hours) and $\left[\mathrm{Me}_{2} \mathrm{NH}_{2}\right] \mathrm{BF}_{4}$ (black). 

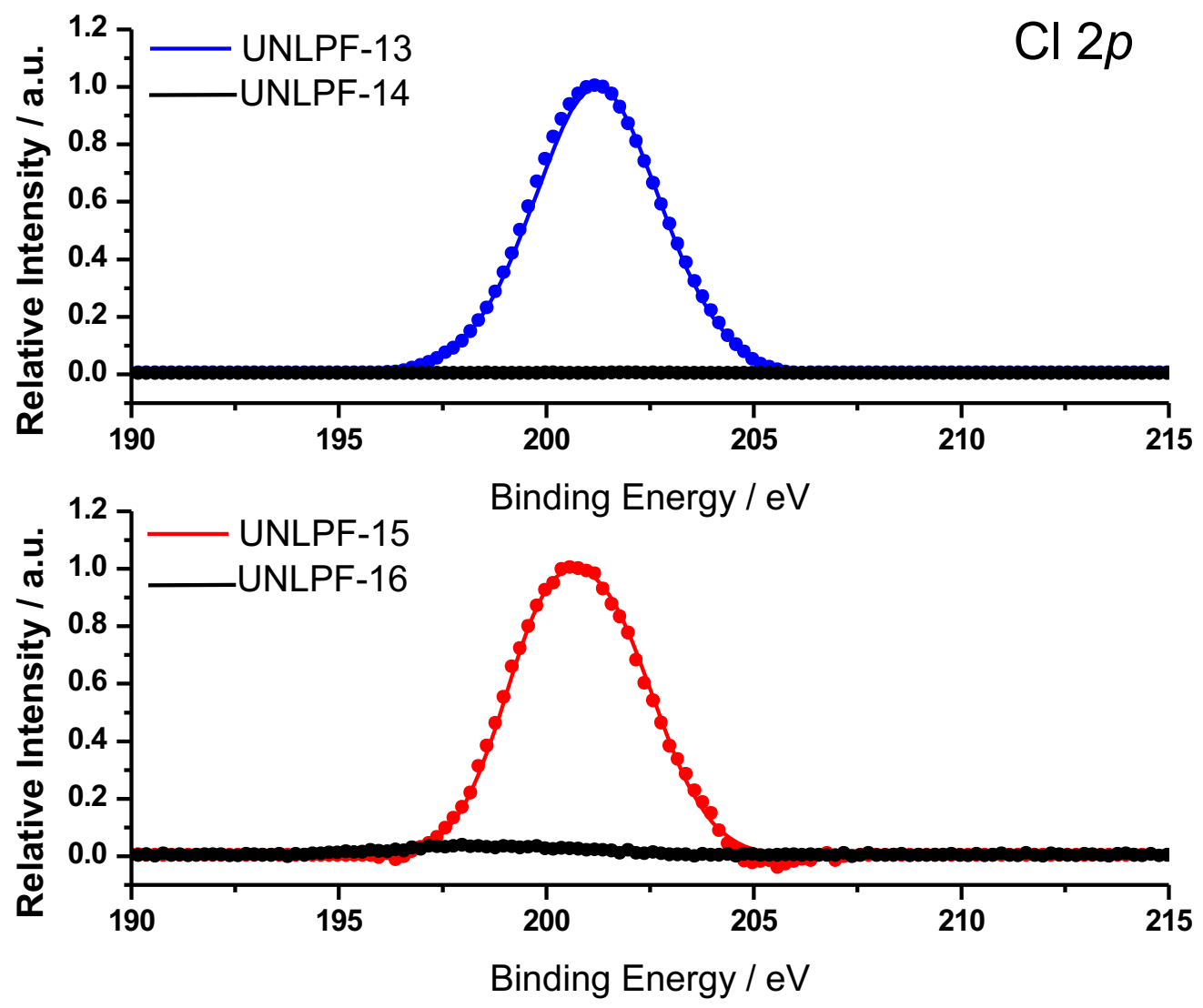

Figure S5. XPS spectra showing the binding energy of $\mathrm{Cl}_{2 p}$ for UNLPF-13 (blue) and UNLPF-15 (red). 

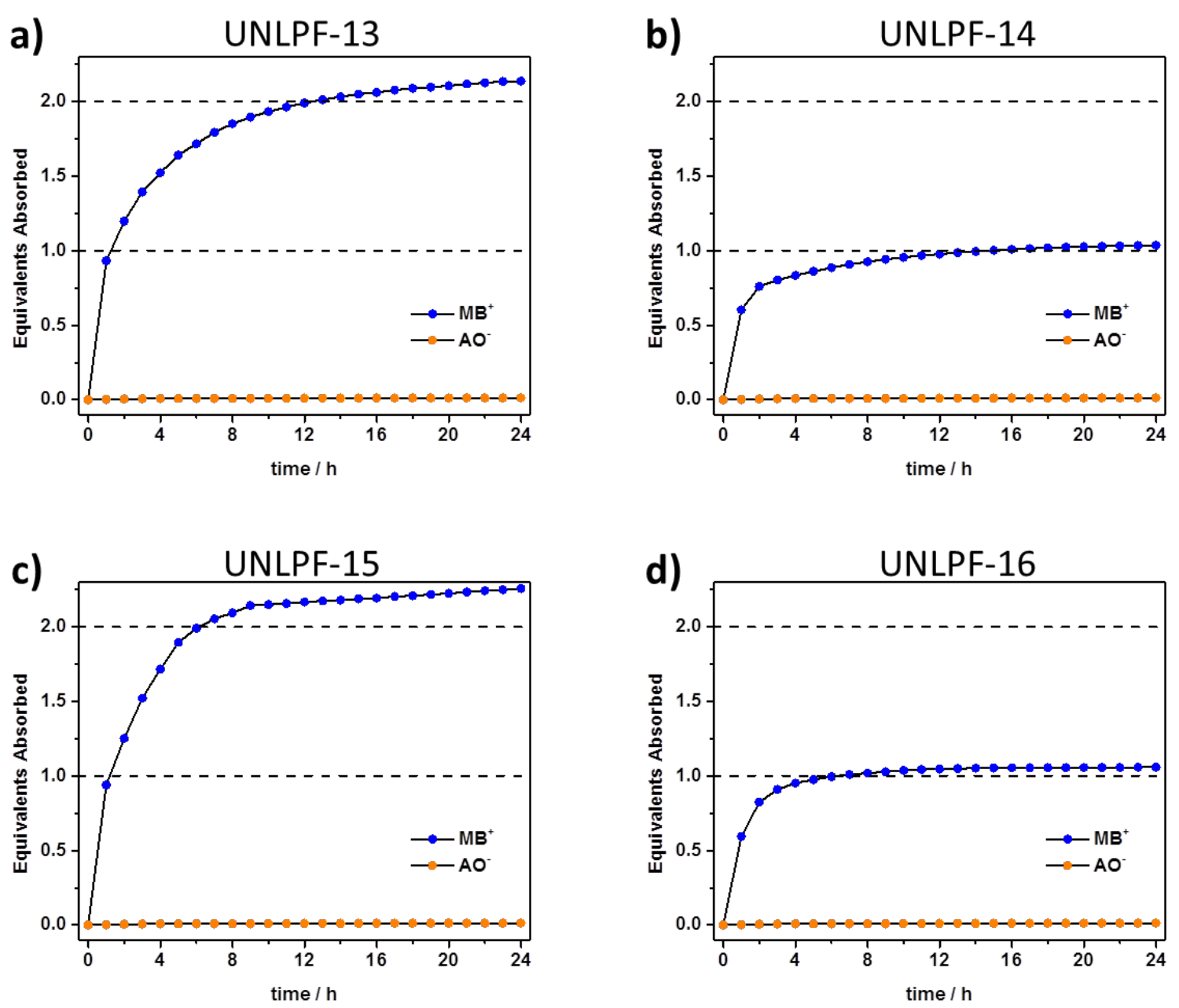

Figure S6. Methylene blue (blue) and acid orange 7 (orange) dye adsorption for samples of UNLPF-13 (a), -14 (b), -15 (c), -16 (d). 
a)

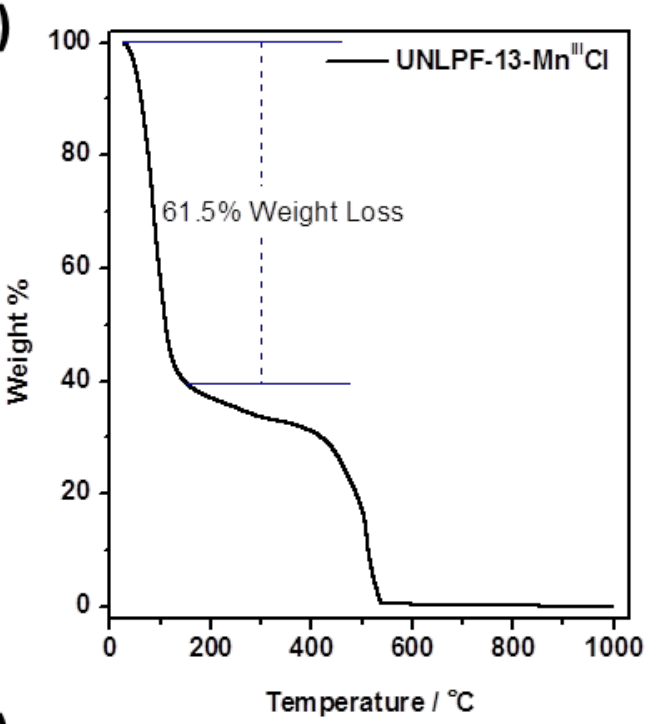

c)

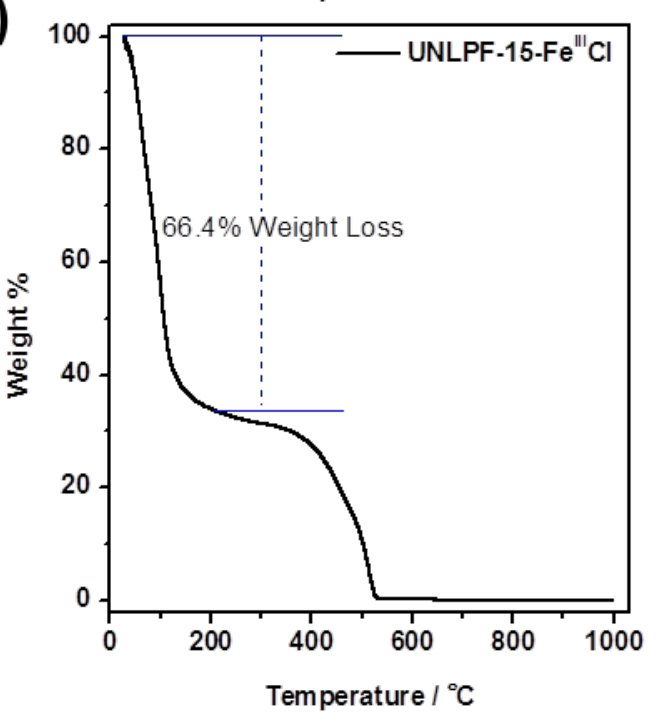

b)
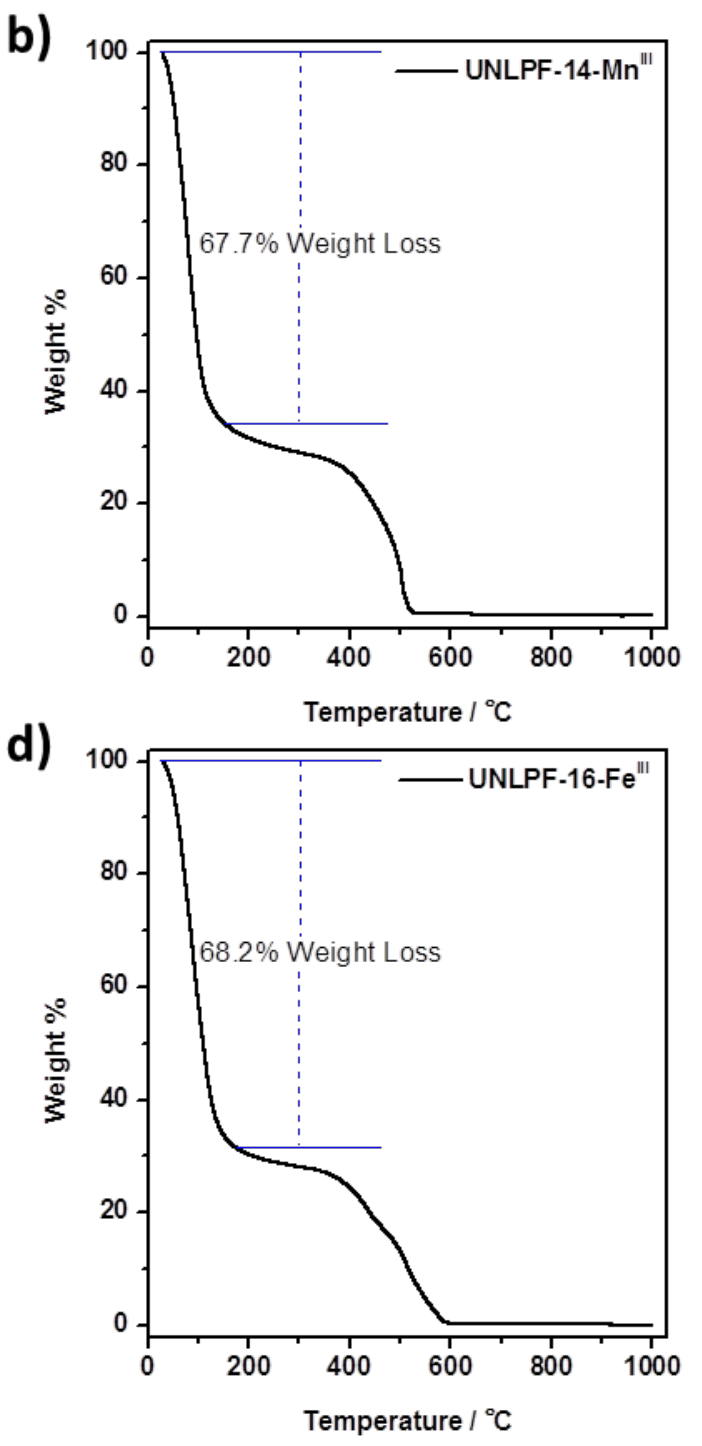

Figure S7. Thermalgravimetric analysis for UNLPF-13- $\mathrm{Mn}{ }^{\prime \prime \prime} \mathrm{Cl}(\mathbf{a}),-14-\mathrm{Mn}^{\prime \prime \prime}(\mathbf{b}),-15-\mathrm{Fe} \mathrm{III}^{\text {IIC }} \mathrm{C}$ ), and $-16-\mathrm{Fe}{ }^{\text {III }}$ (d). 


\section{S3.1 General Procedure for the Preparation of Enyne Substrates}

\section{Procdure A - Step 1}

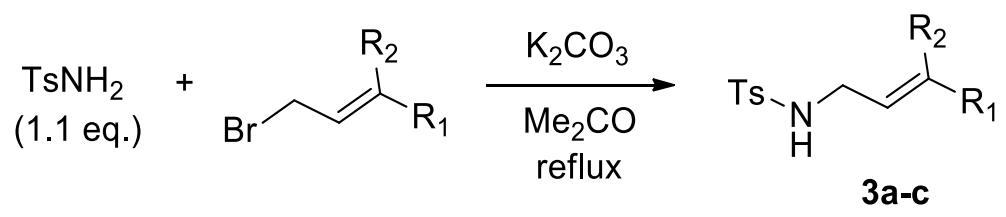

To a stirred suspension of $p$-toluenesulfonamide $(22 \mathrm{mmol})$ and $\mathrm{K}_{2} \mathrm{CO}_{3}(22 \mathrm{mmol})$ in acetone $(20 \mathrm{~mL})$ was added the respective allyl bromide $(20 \mathrm{mmol})$. The reaction mixture was then refluxed at $60^{\circ} \mathrm{C}$ for 30 hours. The reaction was then cooled to room temperature and poured onto water $(\sim 30 \mathrm{~mL})$ and extracted with EtOAc $(3 \times 20 \mathrm{~mL})$. The combined organic layer was dried over $\mathrm{MgSO}_{4}$, filtered, and concentrated. The crude product was purified by column chromatography on a silica gel column with an elution of hexanes/ethyl acetate ( $v / v$ 15:1), recrystallized from ethyl ether and hexanes, and further dried in vacuo to give the desired product.

\section{Procdure A - Step 2}<smiles>[R]C([R])=CCN[AsH-]</smiles>

3a-c

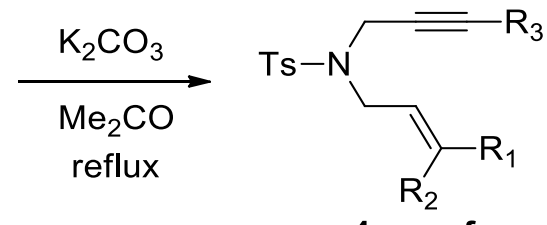

4a-c,e-f

To a stirred suspension of the respective secondary tosyl amide (3) (14 mmol) and $\mathrm{K}_{2} \mathrm{CO}_{3}(21 \mathrm{mmol})$ in acetone $(40 \mathrm{~mL})$ was added the respective $\alpha$-bromo alkyne $(21 \mathrm{mmol})$. The reaction mixture was then refluxed at $60^{\circ} \mathrm{C}$ for $36-48$ hours. The reaction was then cooled to room temperature and poured onto water $(\sim 50 \mathrm{~mL})$ and extracted with EtOAc $(3 \times 20 \mathrm{~mL})$. The combined organic layer was dried over $\mathrm{MgSO}_{4}$, filtered, and concentrated. The crude product was purified by column chromatography on a silica gel column with an elution of hexanes/ethyl acetate ( $v / v 20: 1$ ), recrystallized from ethyl ether and hexanes, and further dried in vacuo to give the desired product. 
Procdure B - Step 1

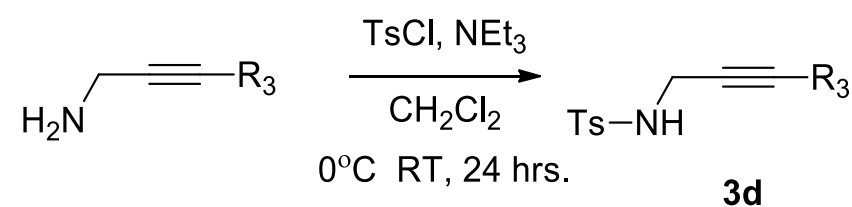

To a stirring solution of propargylamine $(640 \mu \mathrm{L}, 10 \mathrm{mmol})$ and $\mathrm{NEt}_{3}(1.53 \mathrm{~mL}, 11 \mathrm{mmol})$ in $\mathrm{CH}_{2} \mathrm{Cl}_{2}(6.7$ $\mathrm{mL}$ ) at $0^{\circ} \mathrm{C}$, was added dropwise a solution of $\mathrm{TsCl}(2097 \mathrm{mg}, 11 \mathrm{mmol})$ in $\mathrm{CH}_{2} \mathrm{Cl}_{2}(20 \mathrm{~mL})$. The solution was allowed to warm to room temperature and further stirred for an additional 5 hours. The reaction mixture was then poured onto an aqueous solution of saturated $\mathrm{NH}_{4} \mathrm{Cl}(30 \mathrm{~mL})$. The resultant solution was then extraced with $\mathrm{CH}_{2} \mathrm{Cl}_{2}(3 \times 60 \mathrm{~mL})$. The combined organic layers were then washed with brine and dried over $\mathrm{MgSO}_{4}$. The solution was then filtered and concentrated in vacuo. The dried product was then taken up in hexanes and recrystalized via sonication. The pure product was collected by filtration and dried in vacuo at room temperature overnight.

\section{Procdure B - Step 2}

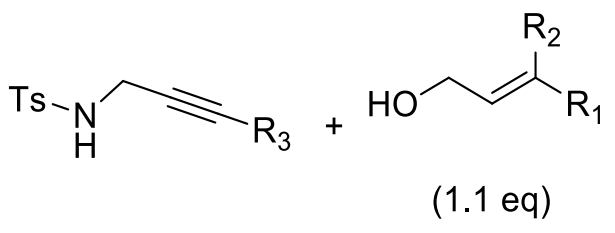

3d

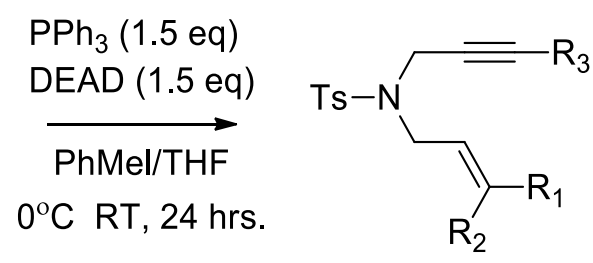

$4 d$

To a degassed solution of the respective secondary tosyl amide (1.44 g, $6.9 \mathrm{mmol}), \mathrm{PPh}_{3}(2.70 \mathrm{~g}, 1.5$ eq.), and the respective allyl alcohol (1.1 eq.) in a mixture of toluene and THF ( $28 \mathrm{~mL}, \mathrm{v} / \mathrm{v} 3: 1$ ) under an $\mathrm{Ar}$ atmosphere stirred at $0^{\circ} \mathrm{C}$ was added dropwise via syrringe diethyl azodicarboxylate (DEAD) (1.62 $\mathrm{mL}$, 1.5 eq.). The reaction mixture was allowed to warm to room temperature and further stirred for 24 hours at room temperature. The crude reaction mixture was then absorbed onto silica and purified by silica gel column chromatography (hexanes/ethyl acetate v/v 15:1). The pure product was then dried in vacuo, recrystalized from hexanes and ethyl ether, and collected via filtration. 


\section{4-methyl-N-(3-methylbut-2-en-1-yl)benzenesulfonamide (3a).}

Prepared according to the general method outlined in step 1 of procedure A. The product was isolated as a crystaline white solid. Yield: $3.49 \mathrm{~g}, 73 \%$.

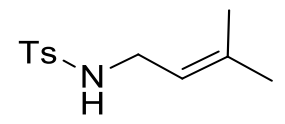

${ }^{1} \mathrm{HNMR}\left(300 \mathrm{MHz}, \mathrm{CDCl}_{3}\right) \delta 7.78(\mathrm{~d}, J=7.5 \mathrm{~Hz}, 2 \mathrm{H}), 7.34(\mathrm{~d}, J=7.5 \mathrm{~Hz}, 2 \mathrm{H}), 5.08$ (tq, $J=3.0 \mathrm{~Hz}, J=1.5$ $\mathrm{Hz}, 1 \mathrm{H}), 4.47(\mathrm{t}, J=2.7 \mathrm{~Hz}, 1 \mathrm{H}), 3.55(\mathrm{t}, J=6.6 \mathrm{~Hz}, 2 \mathrm{H}), 2.45(\mathrm{~s}, 3 \mathrm{H}), 1.64(\mathrm{~s}, 3 \mathrm{H}), 1.55(\mathrm{~s}, 3 \mathrm{H})$.

${ }^{13} \mathrm{CNMR}\left(75 \mathrm{MHz}, \mathrm{CDCl}_{3}\right) \delta 143.33,137.56,137.04,129.63,127.21,118.89,41.04,25.55,21.53,17.77$.

HR-EI MS found for $\left[\mathrm{M}^{+\cdot}\right] \mathrm{m} / \mathrm{z239.0990;}$ calculated for $\left[\mathrm{M}^{++}\right] \mathrm{m} / \mathrm{z239.080.}$

\section{(E)-N-(but-2-en-1-yl)-4-methylbenzenesulfonamide (3b).}

Prepared according to the general method outlined in step 1 of procedure $A$. The product was isolated as a crystaline white solid. Yield: $2.93 \mathrm{~g}, 65 \%$.

Ts<smiles>C/C=C/CNC</smiles>

${ }^{1} \mathrm{HNMR}\left(300 \mathrm{MHz}, \mathrm{CDCl}_{3}\right) \delta 7.78(\mathrm{~d}, J=8.1 \mathrm{~Hz}, 2 \mathrm{H}), 7.34(\mathrm{~d}, J=8.1 \mathrm{~Hz}, 2 \mathrm{H}), 5.58(\mathrm{~m}, 1 \mathrm{H}), 5.37(\mathrm{~m}, 1 \mathrm{H})$, $4.37(\mathrm{t}, J=5.7 \mathrm{~Hz}, 1 \mathrm{H}), 4.48(\mathrm{tt}, J=6.3 \mathrm{~Hz}, J=1.2 \mathrm{~Hz}, 2 \mathrm{H}), 2.45(\mathrm{~s}, 3 \mathrm{H}), 1.64(\mathrm{dd}, J=6.0 \mathrm{~Hz}, J=1.5 \mathrm{~Hz}, 3 \mathrm{H})$.

${ }^{13} \mathrm{CNMR}\left(75 \mathrm{MHz}, \mathrm{CDCl}_{3}\right)$ 8143.37, 137.08, 129.72, 129.67, 127.19, 125.66, 45.34, 21.52, 17.60.

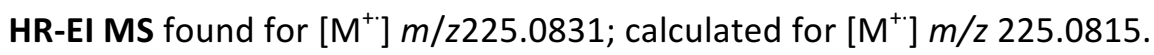

\section{N-allyl-4-methylbenzenesulfonamide (3c).}

Prepared according to the general method outlined in step 1 of procedure $A$. The product was isolated as a crystaline white solid. Yield: $2.62 \mathrm{~g}, 62 \%$.<smiles>C=CCN[TeH]</smiles>

${ }^{1} \mathrm{HNMR}\left(300 \mathrm{MHz}, \mathrm{CDCl}_{3}\right) \delta 7.79(\mathrm{~d}, J=8.4 \mathrm{~Hz}, 2 \mathrm{H}), 7.35(\mathrm{~d}, J=7.8 \mathrm{~Hz}, 2 \mathrm{H}), 5.76(\mathrm{~m}, 1 \mathrm{H}), 5.22(\mathrm{~m}, 1 \mathrm{H})$, $5.14(\mathrm{~m}, 1 \mathrm{H}), 4.48(\mathrm{~m}, 1 \mathrm{H}), 3.62(\mathrm{~m}, 2 \mathrm{H}), 2.46(\mathrm{~s}, 3 \mathrm{H})$.

${ }^{13} \mathrm{CNMR}\left(75 \mathrm{MHz}, \mathrm{CDCl}_{3}\right) \delta 143.55,136.97,133.00,129.75,127.17,117.76,45.81,21.54$.

HR-EI MS found for $\left[\mathrm{M}^{+\cdot}\right] \mathrm{m} / \mathrm{z}$ 211.0673; calculated for $\left[\mathrm{M}^{+}\right] \mathrm{m} / \mathrm{z} 211.0667$. 


\section{4-methyl-N-(prop-2-yn-1-yl)benzenesulfonamide (3d).}

Prepared according to the general method outlined in step 1 of procedure $B$. The product was isolated as a crystaline white solid. Yield: $1.67 \mathrm{~g}, 80 \%$.<smiles>C#CCN[As]</smiles>

${ }^{1} \mathrm{HNMR}\left(300 \mathrm{MHz}, \mathrm{CDCl}_{3}\right) \delta 7.81(\mathrm{~d}, J=8.1 \mathrm{~Hz}, 2 \mathrm{H}), 7.34(\mathrm{~d}, J=8.1 \mathrm{~Hz}, 2 \mathrm{H}), 5.06(\mathrm{br} \mathrm{s}, 1 \mathrm{H}), 3.83(\mathrm{~s}, 2 \mathrm{H})$, $2.44(\mathrm{~s}, 3 \mathrm{H}), 2.11(\mathrm{t}, J=2.4 \mathrm{~Hz}, 1 \mathrm{H})$.

${ }^{13} \mathrm{CNMR}\left(75 \mathrm{MHz}, \mathrm{CDCl}_{3}\right) \delta 143.83,136.51,129.71,127.41,78.04,72.96,32.83,21.57$.

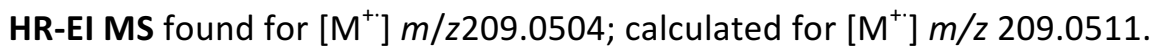

\section{4-methyl-N-(3-methylbut-2-en-1-yl)-N-(prop-2-yn-1-yl)benzenesulfonamide (4a).}

Prepared according to the general method outlined in step 2 of procedure A. The product was isolated as a crystaline white solid. Yield: $3.68 \mathrm{~g}, 95 \%$.

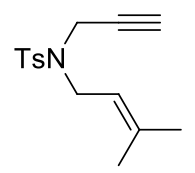

${ }^{1} \mathrm{HNMR}\left(300 \mathrm{MHz}, \mathrm{CDCl}_{3}\right) 87.77(\mathrm{~d}, J=6.3 \mathrm{~Hz}, 2 \mathrm{H}), 7.32(\mathrm{~d}, J=6.0 \mathrm{~Hz}, 2 \mathrm{H}), 5.12(\mathrm{~m}, 1 \mathrm{H}), 4.09(\mathrm{~d}, J=1.8 \mathrm{~Hz}$, $2 \mathrm{H}), 3.84(\mathrm{~d}, J=5.7 \mathrm{~Hz}, 2 \mathrm{H}), 2.44(\mathrm{~s}, 3 \mathrm{H}), 2.00(\mathrm{t}, J=1.8 \mathrm{~Hz}, 1 \mathrm{H}), 1.74(\mathrm{~s}, 3 \mathrm{H}), 1.69(\mathrm{~s}, 3 \mathrm{H})$.

${ }^{13} \mathrm{CNMR}\left(75 \mathrm{MHz}, \mathrm{CDCl}_{3}\right) \delta 143.41,139.03,136.12,129.40,127.78,117.91,77.03,73.43,43.93,35.40$, $25.86,21.53,17.84$.

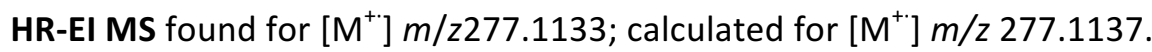

\section{(E)-N-(but-2-en-1-yl)-4-methyl-N-(prop-2-yn-1-yl)benzenesulfonamide (4b).}

Prepared according to the general method outlined in step 2 of procedure $A$. The product was isolated as a crystaline white solid. Yield: $3.13 \mathrm{~g}, 85 \%$.

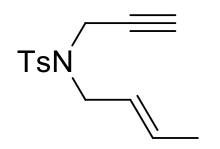

${ }^{1} \mathrm{HNMR}\left(300 \mathrm{MHz}, \mathrm{CDCl}_{3}\right) \delta 7.75(\mathrm{~d}, J=6.0 \mathrm{~Hz}, 2 \mathrm{H}), 7.32(\mathrm{~d}, J=6.3 \mathrm{~Hz}, 2 \mathrm{H}), 5.71(\mathrm{~m}, 1 \mathrm{H}), 5.37(\mathrm{~m}, 1 \mathrm{H})$, $4.09(\mathrm{~d}, J=1.8 \mathrm{~Hz}, 2 \mathrm{H}), 3.78(\mathrm{~d}, J=5.1 \mathrm{~Hz}, 2 \mathrm{H}), 2.44(\mathrm{~s}, 3 \mathrm{H}), 2.09(\mathrm{~s}, 1 \mathrm{H}), 1.71(\mathrm{~d}, J=4.8 \mathrm{~Hz}, 2 \mathrm{H})$. 
${ }^{13} \mathrm{CNMR}\left(75 \mathrm{MHz}, \mathrm{CDCl}_{3}\right) \delta 143.45,136.13,131.70,129.43,127.77,127.52,124.49,73.52,48.28,35.45$, $21.55,17.72$.

HR-EI MS found for $\left[\mathrm{M}^{++}\right] \mathrm{m} / \mathrm{z263.1001;}$ calculated for $\left[\mathrm{M}^{++}\right] \mathrm{m} / \mathrm{z} 263.0980$.

\section{$\mathrm{N}$-allyl-4-methyl-N-(prop-2-yn-1-yl)benzenesulfonamide (4c).}

Prepared according to the general method outlined in step 2 of procedure $A$. The product was isolated as a crystaline white solid. Yield: $2.34 \mathrm{~g}, 67 \%$.

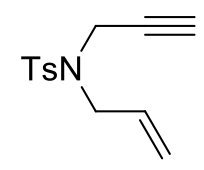

${ }^{1} \mathrm{HNMR}\left(300 \mathrm{MHz}, \mathrm{CDCl}_{3}\right) \delta 7.77(\mathrm{~d}, J=8.1 \mathrm{~Hz}, 2 \mathrm{H}), 7.33(\mathrm{~d}, J=8.1 \mathrm{~Hz}, 2 \mathrm{H}), 5.77(\mathrm{~m}, 1 \mathrm{H}), 5.34(\mathrm{~m}, 1 \mathrm{H})$, $5.25(\mathrm{~m}, 1 \mathrm{H}), 4.09(\mathrm{~d}, J=1.8 \mathrm{~Hz}, 2 \mathrm{H}), 3.86(\mathrm{~d}, J=6.3 \mathrm{~Hz}, 2 \mathrm{H}), 2.45(\mathrm{~s}, 3 \mathrm{H}), 2.02(\mathrm{~s}, 1 \mathrm{H})$.

${ }^{13} \mathrm{CNMR}\left(75 \mathrm{MHz}, \mathrm{CDCl}_{3}\right) \delta 143.58,136.02,131.88,129.50,127.77,120.03,76.51,73.70,48.99,35.75$, 21.56 .

HR-EI MS found for $\left[\mathrm{M}^{++}\right] \mathrm{m} / \mathrm{z} 249.0828$; calculated for $\left[\mathrm{M}^{++}\right] \mathrm{m} / \mathrm{z} 249.0824$.

\section{$\mathrm{N}$-cinnamyl-4-methyl-N-(prop-2-yn-1-yl)benzenesulfonamide (4d).}

Prepared according to the general method outlined in step 2 of procedure A. The product was isolated as a crystaline white solid. Yield: $2.02 \mathrm{~g}, 90 \%$.

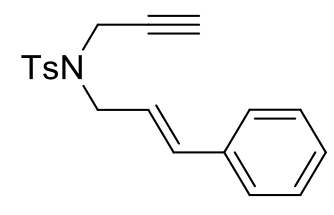

${ }^{1} \mathrm{HNMR}\left(300 \mathrm{MHz}, \mathrm{CDCl}_{3}\right) \delta 7.81(\mathrm{~d}, J=8.4 \mathrm{~Hz}, 2 \mathrm{H}), 7.32(\mathrm{~m}, 7 \mathrm{H}), 6.63(\mathrm{~d}, J=15.9 \mathrm{~Hz}, 1 \mathrm{H}), 6.11(\mathrm{~m}, 1 \mathrm{H})$, $4.16(\mathrm{~d}, J=2.4 \mathrm{~Hz}, 2 \mathrm{H}), 4.03(\mathrm{dd}, J=6.9 \mathrm{~Hz}, J=0.9 \mathrm{~Hz}, 2 \mathrm{H}), 2.46(\mathrm{~s}, 3 \mathrm{H}), 2.08(\mathrm{t}, J=2.7 \mathrm{~Hz}, 1 \mathrm{H})$.

${ }^{13} \mathrm{CNMR}\left(75 \mathrm{MHz}, \mathrm{CDCl}_{3}\right) \delta 143.63,136.12,136.08,134.94,129.54,128.64,128.09,127.82,126.57$, $122.93,76.63,73.85,48.60,35.91,21.58$.

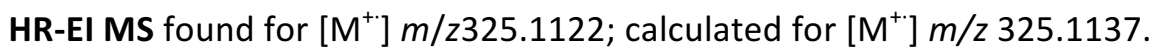




\section{$N$-(but-2-yn-1-yl)-4-methyl-N-(3-methylbut-2-en-1-yl)benzenesulfonamide (4e).}

Prepared according to the general method outlined in step 2 of procedure A. The product was isolated as a crystaline white solid. Yield: $3.87 \mathrm{~g}, 95 \%$.

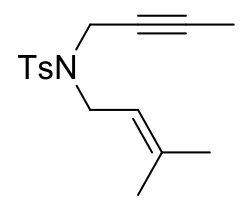

${ }^{1} \mathrm{HNMR}\left(300 \mathrm{MHz}, \mathrm{CDCl}_{3}\right) \delta 7.75(\mathrm{~d}, J=8.1 \mathrm{~Hz}, 2 \mathrm{H}), 7.30(\mathrm{~d}, J=8.1 \mathrm{~Hz}, 2 \mathrm{H}), 5.09(\mathrm{t}, J=7.2 \mathrm{~Hz}, 1 \mathrm{H}), 3.98(\mathrm{~d}$, $J=2.4 \mathrm{~Hz}, 2 \mathrm{H}), 3.78(J=7.2 \mathrm{~Hz}, 2 \mathrm{H}), 2.42(\mathrm{~s}, 3 \mathrm{H}), 1.71(\mathrm{~s}, 3 \mathrm{H}) 1.66(\mathrm{~s}, 3 \mathrm{H}), 1.52(\mathrm{~s}, 3 \mathrm{H})$.

${ }^{13} \mathrm{CNMR}\left(75 \mathrm{MHz}, \mathrm{CDCl}_{3}\right) \delta 143.09$ 138.60, 136.23, 129.17, 127.90, 118.20, 81.25, 72.03, 43.89, 35.96, $25.84,21.48,17.79,3.19$.

HR-EI MS found for [ $\left.\mathrm{M}^{++}\right] \mathrm{m} / \mathrm{z} 291.1306$; calculated for $\left[\mathrm{M}^{++}\right] \mathrm{m} / \mathrm{z} 291.1293$.

\section{$N$-allyl-N-(but-2-yn-1-yl)-4-methylbenzenesulfonamide(4f).}

Prepared according to the general method outlined in step 2 of procedure A. The product was isolated as a crystaline white solid. Yield: $3.02 \mathrm{~g}, 82 \%$.

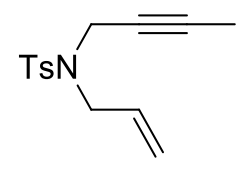

${ }^{1} \mathrm{HNMR}\left(300 \mathrm{MHz}, \mathrm{CDCl}_{3}\right) \delta 7.77(\mathrm{~m}, 2 \mathrm{H}), 7.29(\mathrm{~m}, 2 \mathrm{H}), 5.75(\mathrm{~m}, 1 \mathrm{H}), 5.31(\mathrm{~m}, 1 \mathrm{H}) 5.25(\mathrm{~m}, 1 \mathrm{H}), 4.05(\mathrm{~d}, J=$ $1.5 \mathrm{~Hz}, 2 \mathrm{H}), 3.82(\mathrm{~d}, J=3.9 \mathrm{~Hz}, 2 \mathrm{H}), 2.46(\mathrm{~s}, 2 \mathrm{H}), 1.57(\mathrm{~s}, 3 \mathrm{H})$.

${ }^{13} \mathrm{CNMR}\left(75 \mathrm{MHz}, \mathrm{CDCl}_{3}\right) \delta 143.34,136.35,132.37,129.36,128.01,119.64,81.62,71.77,49.10,36.43$, $21.62,3.34$.

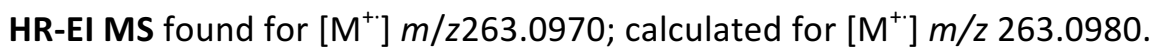




\section{S3.2 General Procedure for the [2+1] Enyne Cycloisomerizations}
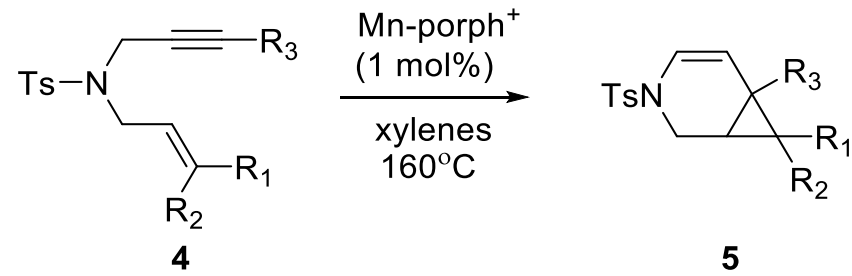

A degassed solution of the respective enyne $(0.2 \mathrm{mmol})$ in xylenes $(1.6 \mathrm{~mL})$ was added to screw cap vial equipped with a stir bar and UNLPF-14 ( $5 \mathrm{mg}$ ). The vial was sealed and heated at $160^{\circ} \mathrm{C}$. Reaction progress was monitored by TLC (hexanes/ethyl acetate $\mathrm{v} / \mathrm{v}$ 30:1). Upon disappearance of the enyne starting material, the reaction mixture was cooled to room temperature and concentrated in vacuo. The crude reaction mixture was purified by flash chromatography using a silica gel column. The pure product was dried in vacuo and recyrstalized from hexanes and diethyl ether.

\section{7, 7-dimethyl-3-tosyl-3-azabicyclo[4-1.0]hept-4-ene (5a).}

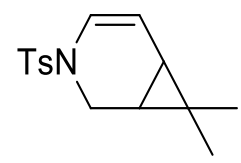

${ }^{1} \mathrm{H}$ NMR $\left(300 \mathrm{MHz}, \mathrm{CDCl}_{3}\right) \delta 7.66$ (d, J = 8.3 Hz, 2H, TosH-2,6), 7.29 (d, J = 8.1 Hz, 2H, Tos H-3,5), 6.59 (d, J $=8.4 \mathrm{~Hz}, 1 \mathrm{H}, \mathrm{H}-4), 5.06(\mathrm{dd}, J=8.4,4.7 \mathrm{~Hz}, 1 \mathrm{H}, \mathrm{H}-5), 3.48(\mathrm{dd}, J=12.2,1.3 \mathrm{~Hz}, 1 \mathrm{H}, \mathrm{H}-2 \mathrm{a}), 3.36(\mathrm{dd}, J=$ 12.2, $5.8 \mathrm{~Hz}, 1 \mathrm{H}, \mathrm{H}-2 \mathrm{~b}), 2.41\left(\mathrm{~s}, 3 \mathrm{H}, \mathrm{Tos}-\mathrm{CH}_{3}\right), 1.07-1.00(\mathrm{~m}, 1 \mathrm{H}, \mathrm{H}-1), 1.05\left(\mathrm{~s}, 3 \mathrm{H}, 7-\mathrm{CH}_{3} \mathrm{a}\right), 0.97$ (dd, $J=$ 8.8, $4.7 \mathrm{~Hz}, 1 \mathrm{H}, \mathrm{H}-6), 0.73\left(\mathrm{~s}, 3 \mathrm{H}, 7-\mathrm{CH}_{3} \mathrm{~b}\right)$.

${ }^{13} \mathrm{C}$ NMR $\left(75 \mathrm{MHz}, \mathrm{CDCl}_{3}\right) \delta 143.79,134.82,129.79,127.27,122.46,104.43,38.73,27.47,25.54,21.98$, $21.67,18.81,14.15$.

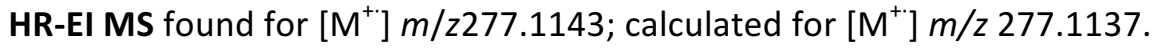

$(1 R, 6 S, 7 S)$-7-methyl-3-tosyl-3-azabicyclo[4.1.0]hept-4-ene(5b).

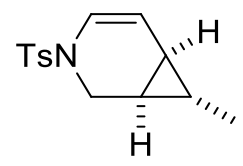

${ }^{1} \mathrm{H}$ NMR $\left(400 \mathrm{MHz}, \mathrm{CDCl}_{3}\right) \delta 7.64$ (d, J = 8.3 Hz, 2H, Tos H-2,6), 7.30 (d, J = 8.0 Hz, 2H, Tos H-3,5), 6.29 (d, J $=8.1 \mathrm{~Hz}, 1 \mathrm{H}, \mathrm{H}-4), 5.41(\mathrm{dd}, J=8.1,5.4 \mathrm{~Hz}, 1 \mathrm{H}, \mathrm{H}-5), 3.88$ (dd, $J=11.5,0.9 \mathrm{~Hz}, 1 \mathrm{H}, \mathrm{H}-2 \mathrm{a}), 2.95$ (dd, $J=$ 11.5, 3.0 Hz, 1H, H-2b), $2.42\left(\mathrm{~s}, 3 \mathrm{H}, \mathrm{Tos}-\mathrm{CH}_{3}\right), 1.25-1.18(\mathrm{~m}, 1 \mathrm{H}, \mathrm{H}-1), 0.96\left(\mathrm{~d}, J=6.1 \mathrm{~Hz}, 3 \mathrm{H}, 7-\mathrm{CH}_{3}\right), 0.84$ (ddd, $=8.7,5.2,3.8 \mathrm{~Hz}, 1 \mathrm{H}, \mathrm{H}-6), 0.75-0.66(\mathrm{~m}, 1 \mathrm{H}, \mathrm{H}-7)$. 
${ }^{13} \mathrm{C}$ NMR $\left(101 \mathrm{MHz}, \mathrm{CDCl}_{3}\right) \delta 143.72,134.97,129.82,127.20,120.85,111.98,40.84,26.51,21.82,21.67$, $17.56,15.56$.

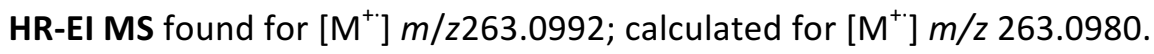

\section{3-tosyl-3-azabicyclo[4.1.0]hept-4-ene(5c).}

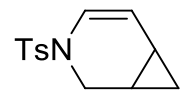

${ }^{1}$ H NMR $\left(300 \mathrm{MHz}, \mathrm{CDCl}_{3}\right) \delta 7.65$ (d, J = 8.3 Hz, 2H, Tos H-2,6), 7.31 (d, J = 8.1 Hz, 2H, Tos H-3,5), 6.33 (d, J $=8.1 \mathrm{~Hz}, 1 \mathrm{H}, \mathrm{H}-4), 5.40(\mathrm{dd}, J=8.1,5.4 \mathrm{~Hz}, 1 \mathrm{H}, \mathrm{H}-5), 3.88(\mathrm{~d}, J=11.7 \mathrm{~Hz}, 1 \mathrm{H}, \mathrm{H}-2 \mathrm{a}), 3.05$ (dd, J = 11.5, 2.9 $\mathrm{Hz}, 1 \mathrm{H}, \mathrm{H}-2 \mathrm{~b}), 2.42\left(\mathrm{~s}, 3 \mathrm{H}\right.$, Tos- $\left.\mathrm{CH}_{3}\right), 1.54-1.47(\mathrm{~m}, 1 \mathrm{H}, \mathrm{H}-1), 1.19-1.06(\mathrm{~m}, 1 \mathrm{H}, \mathrm{H}-6), 0.84-0.72(\mathrm{~m}, 1 \mathrm{H}$, H7-a), $0.39-0.31$ (m, 1H, H7-b).

${ }^{13} \mathrm{C}$ NMR $\left(75 \mathrm{MHz}, \mathrm{CDCl}_{3}\right) \delta 143.75,135.04,129.86,127.19,121.25,112.20,41.01,21.69,18.63,13.55$, 7.24 .

HR-EI MS found for $\left[\mathrm{M}^{+*}\right] \mathrm{m} / \mathrm{z}$ 249.0826; calculated for $\left[\mathrm{M}^{+}\right] \mathrm{m} / \mathrm{z} 249.0824$.

(1S,6S,7S)-7-phenyl-3-tosyl-3-azabicyclo[4.1.0]hept-4-ene (5d).

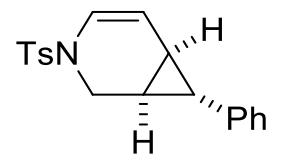

${ }^{1} \mathrm{H}$ NMR $\left(300 \mathrm{MHz}, \mathrm{CDCl}_{3}\right) \delta 7.70(\mathrm{~d}, J=8.1 \mathrm{~Hz}, 2 \mathrm{H}$, Tos H-2,6), $7.36(\mathrm{~d}, J=8.0 \mathrm{~Hz}, 2 \mathrm{H}$, Tos H-3,5), $7.22(\mathrm{t}, J$ $=7.3 \mathrm{~Hz}, 2 \mathrm{H}, \mathrm{Ph} \mathrm{H}-3,5), 7.14$ (t, J = 7.2 Hz, 1H, Ph H-4), 6.80 (d, J = 7.3 Hz, 2H, Ph H-2,6), 6.43 (d, J = 8.0 Hz, $1 \mathrm{H}, \mathrm{H}-4), 5.51(\mathrm{dd}, J=7.8,5.5 \mathrm{~Hz}, 1 \mathrm{H}, \mathrm{H}-5), 4.03(\mathrm{~d}, J=12.0 \mathrm{~Hz}, 1 \mathrm{H}, \mathrm{H}-2 \mathrm{a}), 3.16$ (dd, J = 12.0, 2.6 Hz, $1 \mathrm{H}, \mathrm{H}-$ 2b), $2.48\left(\mathrm{~s}, 3 \mathrm{H}\right.$, Tos- $\left.\mathrm{CH}_{3}\right), 1.96-1.86(\mathrm{~m}, 1 \mathrm{H}, \mathrm{H}-1), 1.63(\mathrm{t}, \mathrm{J}=4.3 \mathrm{~Hz}, 1 \mathrm{H}, \mathrm{H}-7), 1.51-1.43(\mathrm{~m}, 1 \mathrm{H}, \mathrm{H}-6)$.

${ }^{13} \mathrm{C}$ NMR $\left(75 \mathrm{MHz}, \mathrm{CDCl}_{3}\right) \delta 143.94,140.64,134.93,130.04,128.52,127.24,126.04,125.42,121.58$, $111.34,40.32,31.29,29.08,21.70,19.53$.

HR-EI MS found for $\left[\mathrm{M}^{++}\right] \mathrm{m} / \mathrm{z}$ 324.1142; calculated for $\left[\mathrm{M}^{++}\right] \mathrm{m} / \mathrm{z} 325.1137$. 


\section{6,7,7-trimethyl-3-tosyl-3-azabicyclo[4-1.0]hept-4-ene (5e).}

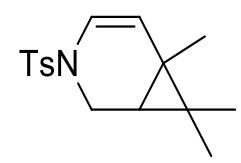

${ }^{1} \mathrm{H}$ NMR $\left(400 \mathrm{MHz}, \mathrm{CDCl}_{3}\right) \delta 7.67(\mathrm{~d}, J=8.1 \mathrm{~Hz}, 2 \mathrm{H}$, Tos H-2,6), $7.30(\mathrm{~d}, J=8.1 \mathrm{~Hz}, 2 \mathrm{H}$, Tos H-3,5), 6.54 (d, J $=8.4 \mathrm{~Hz}, 1 \mathrm{H}, \mathrm{H}-4), 4.86(\mathrm{~d}, J=8.4 \mathrm{~Hz}, 1 \mathrm{H}, \mathrm{H}-5), 3.46$ (dd, $J=12.0,1.2 \mathrm{~Hz}, 1 \mathrm{H}, \mathrm{H}-2 \mathrm{a}$ ), 3.38 (dd, $J=12.1,6.1$ $\mathrm{Hz}, 1 \mathrm{H}, \mathrm{H}-2 \mathrm{~b}), 2.42\left(\mathrm{~s}, 3 \mathrm{H}, \mathrm{Tos}-\mathrm{CH}_{3}\right), 1.12\left(\mathrm{~s}, 3 \mathrm{H}, 6-\mathrm{CH}_{3}\right), 1.07\left(\mathrm{~s}, 3 \mathrm{H} 7-\mathrm{CH}_{3} \mathrm{a}\right), 0.73\left(\mathrm{~s}, 3 \mathrm{H}, 7-\mathrm{CH}_{3} \mathrm{~b}\right), 0.70$ (dd, $J=6.2,1.3 \mathrm{~Hz}, 1 \mathrm{H}, \mathrm{H}-1)$.

${ }^{13} \mathrm{C}$ NMR $\left(101 \mathrm{MHz}, \mathrm{CDCl}_{3}\right) \delta 143.74,134.99,129.78,127.32,121.63,110.67,38.72,28.24,28.01,23.01$, $21.69,19.56,19.19,16.30$.

ESI MS found for $\left[\mathrm{M}^{++}\right] \mathrm{m} / \mathrm{z291.1279;}$ calculated for $\left[\mathrm{M}^{+}\right] \mathrm{m} / \mathrm{z} 291.1293$.

6-methyl-3-tosyl-3-azabicyclo[4.1.0]hept-4-ene (5f).

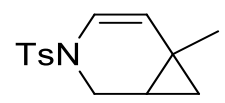

${ }^{1}$ H NMR $\left(400 \mathrm{MHz}, \mathrm{CDCl}_{3}\right) \delta 7.64(\mathrm{~d}, J=8.2 \mathrm{~Hz}, 2 \mathrm{H}$, Tos H-2,6), $7.30(\mathrm{~d}, J=8.2 \mathrm{~Hz}, 2 \mathrm{H}, \operatorname{Tos} \mathrm{H}-3,5), 6.29$ (d, J $=8.1 \mathrm{~Hz}, 1 \mathrm{H}, \mathrm{H}-4), 5.22(\mathrm{~d}, J=8.1 \mathrm{~Hz}, 1 \mathrm{H}, \mathrm{H}-5), 3.83$ (dd, $J=11.3,2.0 \mathrm{~Hz}, 1 \mathrm{H}, \mathrm{H}-2 \mathrm{a}$ ), 3.03 (dd, J = 11.5, 2.7 $\mathrm{Hz}, 1 \mathrm{H}, \mathrm{H}-2 \mathrm{~b}), 2.41\left(\mathrm{~s}, 3 \mathrm{H}, \mathrm{Tos}-\mathrm{CH}_{3}\right), 1.26(\mathrm{td}, J=5.8,2.7 \mathrm{~Hz}, 1 \mathrm{H}, \mathrm{H}-1), 1.09\left(\mathrm{~s}, 3 \mathrm{H}, 6-\mathrm{CH}_{3}\right), 0.57$ (dd, $J=8.4$, $4.4 \mathrm{~Hz}, 1 \mathrm{H}, \mathrm{H}-7 \mathrm{a}), 0.45(\mathrm{t}, J=5.0 \mathrm{~Hz}, 1 \mathrm{H}, \mathrm{H}-7 \mathrm{~b})$.

${ }^{13} \mathrm{C}$ NMR $\left(101 \mathrm{MHz}, \mathrm{CDCl}_{3}\right) \delta 143.73,135.04,129.83,127.16,120.35,116.68,41.09,25.80,23.31,21.66$, $20.95,12.83$.

ESI MS found for $\left[\mathrm{M}^{++}\right] \mathrm{m} / \mathrm{z263.0981;} \mathrm{calculated} \mathrm{for}\left[\mathrm{M}^{+}\right] \mathrm{m} / \mathrm{z} 263.0980$. 


\section{S3.3 Test for Catalyst Heterogeneity}

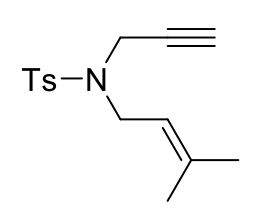

$4 \mathbf{a}$

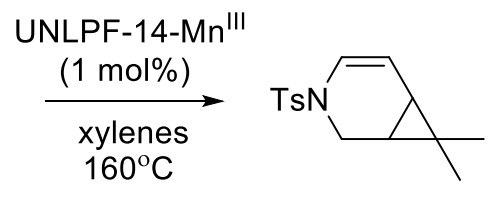

$5 a$

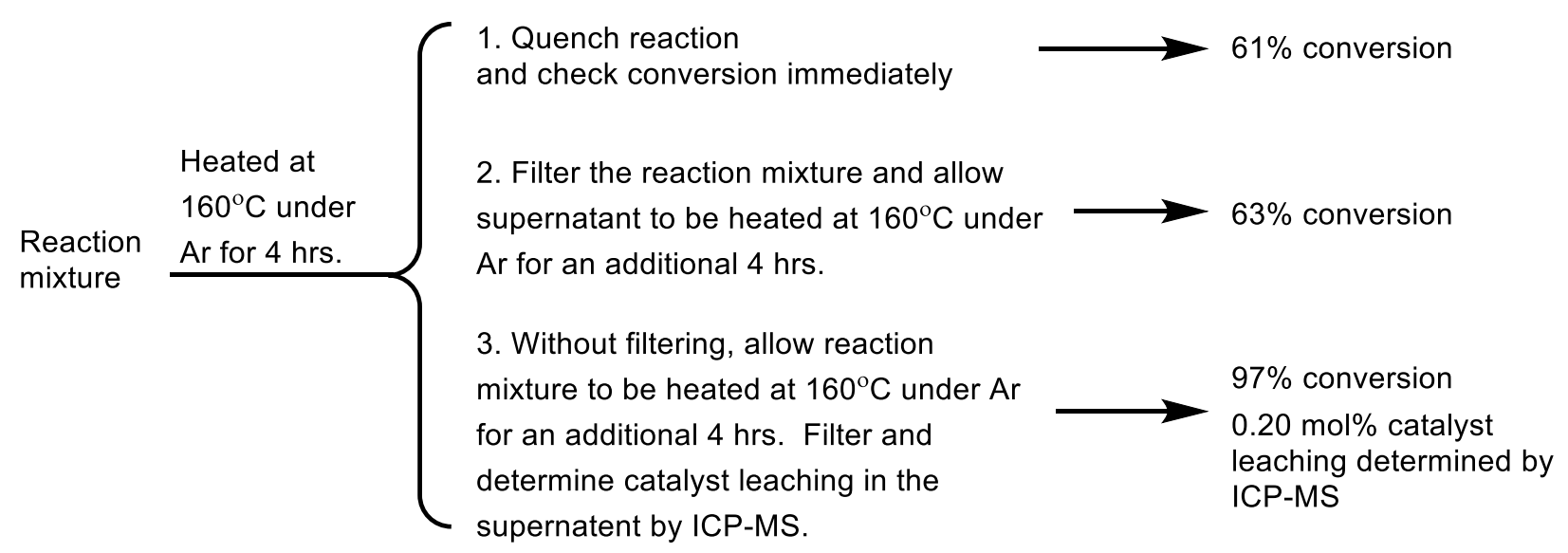

Scheme S3. Heterogeneity test for UNLPF-14-Mn"I catalyzing the [2+1] enyne cycloisomerizations of $4 a$. 


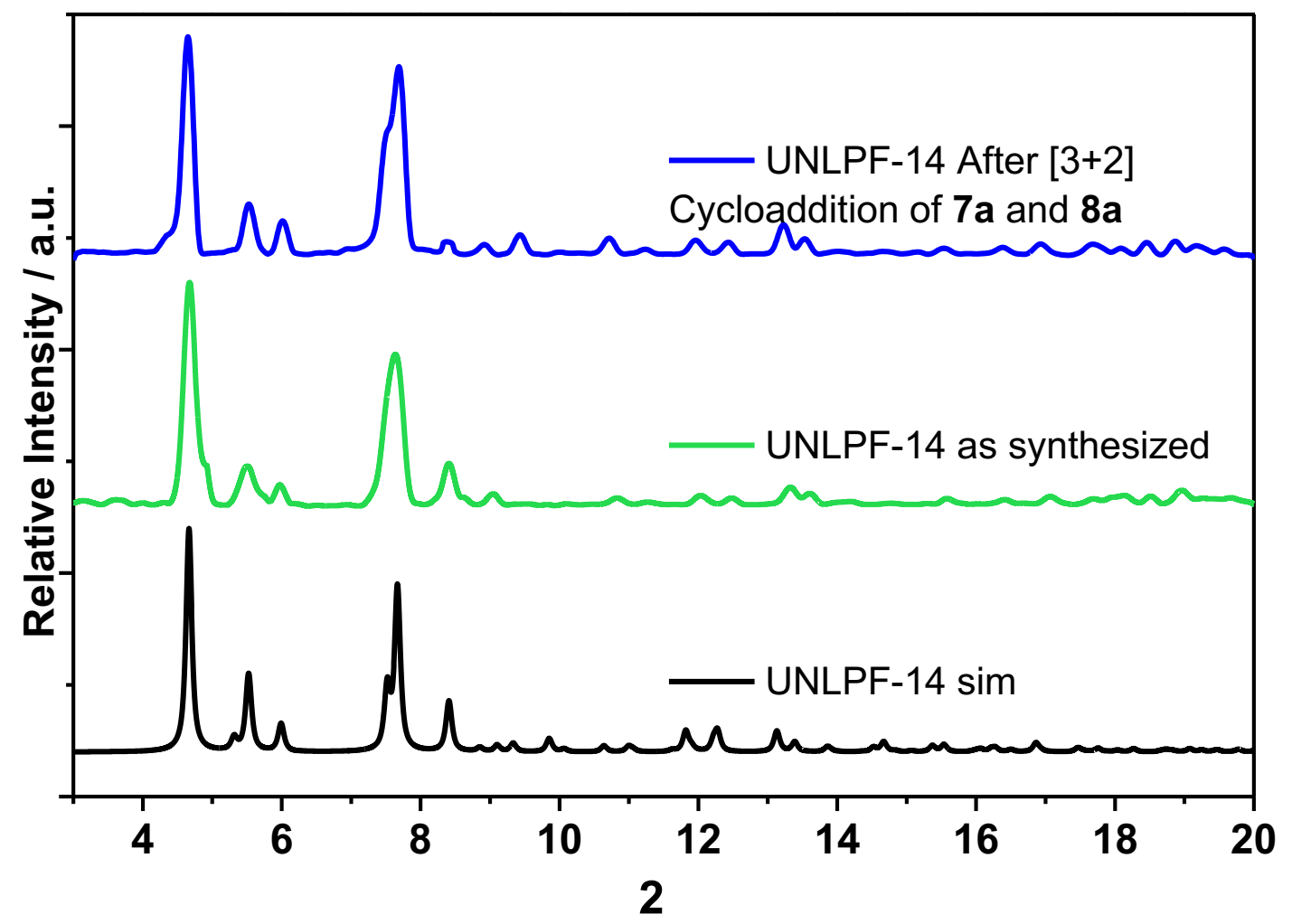

Figure S8. Powder X-ray diffraction patterns of UNLPF-14 simulated (black), as synthesized (green), and after the $[2+1]$ cycloisomerization of enynes (blue). 
S3.4 Proposed Mechanism for the [2+1] Enyne Cycloisomerizations
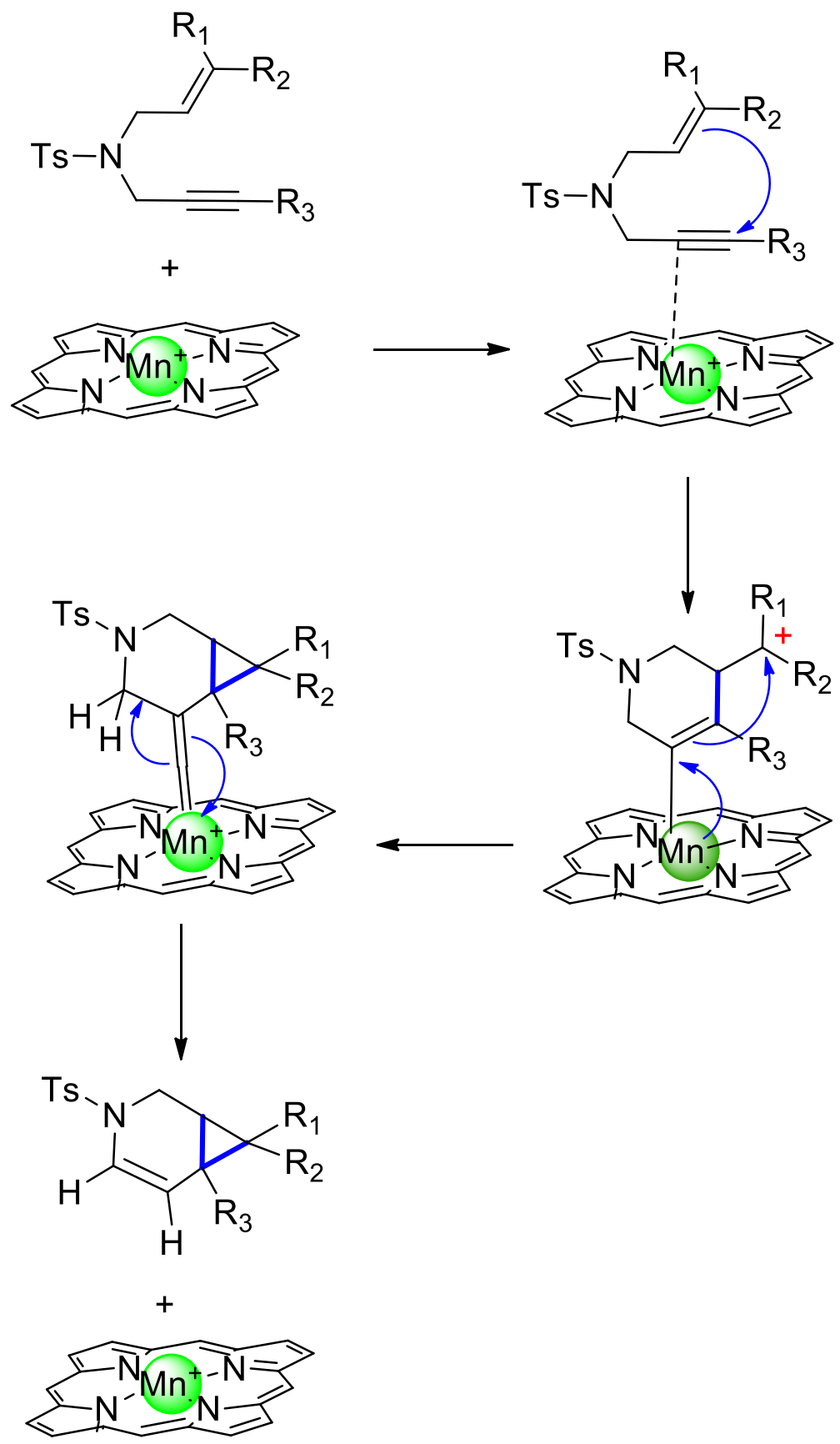

Scheme S4. Proposed mechanism for the $[2+1]$ Cycloisomerization of Enynes. 


\section{S4.1 General Procedure for the Preparation of Aziridine Substrates}

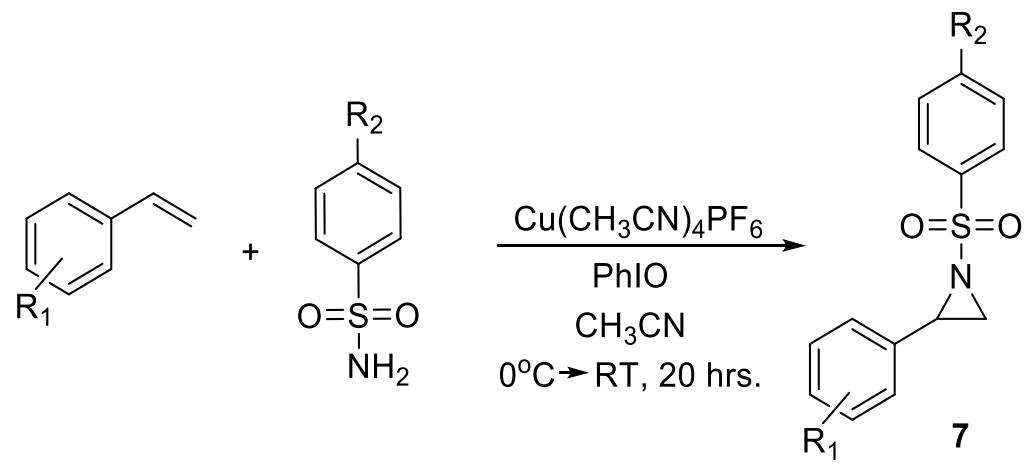

To a degassed solution of $\left[\mathrm{Cu}\left(\mathrm{CH}_{3} \mathrm{CN}\right)_{4}\right] \mathrm{PF}_{6}{ }^{9}(149 \mathrm{mg}, 0.4 \mathrm{mmol})$ and the corresponding alkene $(4.0 \mathrm{mmol})$ in $\mathrm{CH}_{3} \mathrm{CN}(10 \mathrm{~mL})$ in the presence of activated molecular sieves $(4 \AA)$ was added the respective aryl sulfonamide $(5.6 \mathrm{mmol})$ portionwise. Upon complete dissolution of the aryl sulfonamide the reaction was cooled to $0^{\circ} \mathrm{C}$ and $\mathrm{PhIO}^{10,11}(1.23 \mathrm{~g}, 5.6 \mathrm{mmol})$ was added slowly. The reaction mixture was then stirred under argon for 20 hrs. The reaction was then diluted with $\mathrm{CH}_{2} \mathrm{Cl}_{2}(100 \mathrm{~mL})$, filtered through a bed of Celite, and concentrated in vacuo. The crude reaction mixture was then purified by column chromatography on a silica gel column with an elution of hexanes/ethyl acetate $(v / v 6: 1)$ and further dried in vacuo to give the desired product.

\section{2-phenyl-1-tosylaziridine (7a).}

Prepared according to the general procedure. The product was isolated as a white solid. Yield: $0.76 \mathrm{~g}$, $70 \%$.

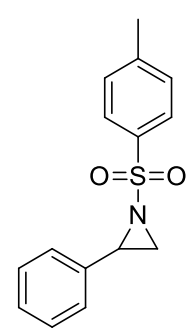

${ }^{1} \mathrm{H}$ NMR $\left(300 \mathrm{MHz}, \mathrm{CDCl}_{3}\right) \delta 7.91(\mathrm{~d}, J=8.1 \mathrm{~Hz}, 2 \mathrm{H}), 7.37(\mathrm{~d}, J=8.1 \mathrm{~Hz}, 2 \mathrm{H}), 7.32(\mathrm{~m}, 3 \mathrm{H}), 7.24(\mathrm{~m}, 2 \mathrm{H})$, $3.81(\mathrm{dd}, J=4.2 \mathrm{~Hz}, J=2.7 \mathrm{~Hz}, 1 \mathrm{H}), 3.02(\mathrm{~d}, J=7.2 \mathrm{~Hz}, 1 \mathrm{H}), 2.46(\mathrm{~s}, 3 \mathrm{H}), 2.42(\mathrm{~d}, J=4.5 \mathrm{~Hz}, 1 \mathrm{H})$.

${ }^{13} \mathrm{C}$ NMR $\left(75 \mathrm{MHz}, \mathrm{CDCl}_{3}\right)$ 8144.66, 135.07, 129.77, 128.58, 128.32, 127.97, 126.58, 41.06, 35.95, 21.67. ESI MS found for $\left[\mathrm{M}^{+\cdot}\right] \mathrm{m} / \mathrm{z} 273.0825$; calculated for $\left[\mathrm{M}^{++}\right] \mathrm{m} / \mathrm{z} 273.0824$. 


\section{2-(perfluorophenyl)-1-tosylaziridine (7b).}

Prepared according to the general procedure. The product was isolated as an off-white solid. Yield: $0.81 \mathrm{~g}, 52 \%$.

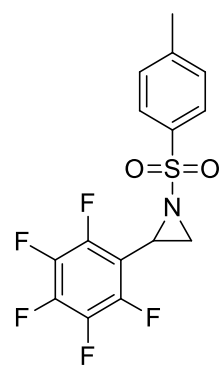

${ }^{1} \mathrm{HNMR}\left(300 \mathrm{MHz}, \mathrm{CDCl}_{3}\right) \delta 7.90(\mathrm{~d}, J=8.4 \mathrm{~Hz}, 2 \mathrm{H}), 7.40(\mathrm{~d}, J=8.1 \mathrm{~Hz}, 2 \mathrm{H}), 3.82(\mathrm{dd}, J=4.5 \mathrm{~Hz}, J=2.7 \mathrm{~Hz}$, $1 \mathrm{H}), 3.08(\mathrm{~d}, J=7.2 \mathrm{~Hz}, 1 \mathrm{H}), 2.83(\mathrm{~d}, J=4.5 \mathrm{~Hz}, 1 \mathrm{H}), 2.49(\mathrm{~s}, 3 \mathrm{H})$.

${ }^{13}$ CNMR $\left(75 \mathrm{MHz}, \mathrm{CDCl}_{3}\right) \delta 147.72,145.26,144.39,143.06,139.43,135.84,134.13,129.85,128.27$, $108.93,32.45,31.89,21.70$.

${ }^{19}$ F NMR $\left(282 \mathrm{MHz} \mathrm{CDCl}_{3}\right) \delta-142.23,-152.68,-161.32$.

ESI MS found for $\left[\mathrm{M}^{+\cdot}\right] \mathrm{m} / \mathrm{z363.0365}$; calculated for $\left[\mathrm{M}^{++}\right] \mathrm{m} / \mathrm{z} 363.0352$.

\section{2-phenyl-1-((4-(trifluoromethyl)phenyl)sulfonyl)aziridine (7f).}

Prepared according to the general procedure. The product was isolated as a white solid. Yield: $0.90 \mathrm{~g}$, $69 \%$.

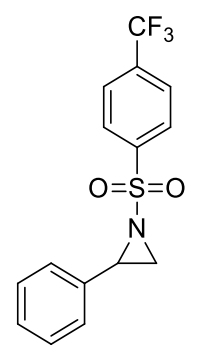

${ }^{1} \mathrm{HNMR}\left(300 \mathrm{MHz}, \mathrm{CDCl}_{3}\right) \delta 8.17(\mathrm{~d}, J=8.4 \mathrm{~Hz}, 2 \mathrm{H}), 7.85(\mathrm{~d}, J=8.4 \mathrm{~Hz}, 2 \mathrm{H}), 7.34(\mathrm{~m}, 3 \mathrm{H}), 7.25(\mathrm{~m}, 2 \mathrm{H}), 3.91$ (dd, $J=7.2 \mathrm{~Hz}, J=4.5 \mathrm{~Hz}, 1 \mathrm{H}$ ), $3.11(\mathrm{~d}, J=7.2 \mathrm{~Hz}, 1 \mathrm{H}), 2.50(\mathrm{~d}, J=4.5 \mathrm{~Hz}, 1 \mathrm{H}$ ).

${ }^{13} \mathrm{CNMR}\left(75 \mathrm{MHz}, \mathrm{CDCl}_{3}\right) \delta 141.80,135.30$ (q, $\left.J=33.3 \mathrm{~Hz}\right), 134.46,128.72,128.65,128.44,126.51$, 126.32(q, $J=3.7 \mathrm{~Hz}), 123.13(q, J=273.2 \mathrm{~Hz}), 41.60,36.41$.

${ }^{19}$ F NMR $\left(282 \mathrm{MHz}, \mathrm{CDCl}_{3}\right) \delta-63.22$.

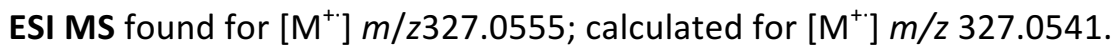




\section{2-(4-chlorophenyl)-1-((4-nitrophenyl)sulfonyl)aziridines (7g).}

Prepared according to the general procedure. The product was isolated as an off-white solid. Yield : $0.61 \mathrm{~g}, 45 \%$.<smiles>O=[N+]([O-])c1ccc(S(=O)(=O)N2CC2c2ccc(Cl)cc2)cc1</smiles>

${ }^{1} \mathrm{HNMR}\left(300 \mathrm{MHz}, \mathrm{CDCl}_{3}\right) \delta 8.43(\mathrm{~d}, J=9.0 \mathrm{~Hz}, 2 \mathrm{H}), 8.22(\mathrm{~d}, J=9.0 \mathrm{~Hz}, 2 \mathrm{H}), 7.34(\mathrm{~d}, J=8.7 \mathrm{~Hz}, 2 \mathrm{H}), 7.19$ (d, $J=8.4 \mathrm{~Hz}, 2 \mathrm{H}), 3.92(\mathrm{dd}, J=7.2 \mathrm{~Hz}, J=4.5 \mathrm{~Hz}, 1 \mathrm{H}) 3.14(\mathrm{~d}, J=7.2 \mathrm{~Hz}, 1 \mathrm{H}), 2.49(\mathrm{~d}, J=4.5 \mathrm{~Hz}, 1 \mathrm{H})$.

${ }^{13} \mathrm{CNMR}\left(75 \mathrm{MHz} \mathrm{CDCl}_{3}\right) \delta 150.77,143.79,134.77,132.77,129.23,129.03,127.81,124.43,41.08,36.73$.

ESI MS found for $\left[\mathrm{M}^{+\cdot}\right] \mathrm{m} / \mathrm{z338.0127}$; calculated for $\left[\mathrm{M}^{++}\right] \mathrm{m} / \mathrm{z} 338.0128$.

\section{S4.2 General Procedure for the [3+2] Cycloaddition of Aziridines and Alkenes}
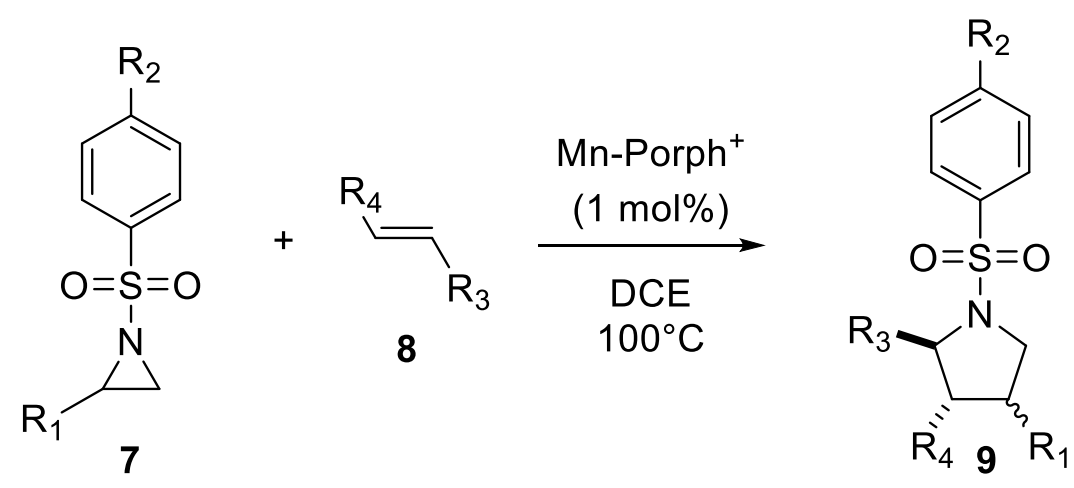

To a screw cap vial equipped with a stir bar was added the respective aziridine $(0.4 \mathrm{mmol})$, alkene (1.2 $\mathrm{mmol})$, and UNLPF-14 $(10 \mathrm{mg})$ in DCE $(1.6 \mathrm{~mL})$. The vial was sealed and the solution was stirred at $100^{\circ} \mathrm{C}$. Upon completion (determined by disappearance of the aziridine on TLC) the reaction mixture was filtered to remove the catalyst and further concentrated in vacuo. The crude product was purified by column chromatography on silica gel (hexanes/EtOAc). The product was dried in vacuo and recrystallized from pentane and $\mathrm{Et}_{2} \mathrm{O}$. In some cases singe crystals could be grown. 


\section{2-(4-chlorophenyl)-4-phenyl-1-tosylpyrrolidine (9a).}

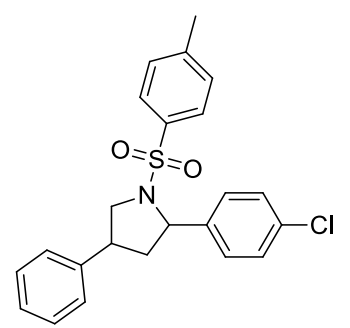

${ }^{1} \mathrm{H}$ NMR, COSY $\left(300 \mathrm{MHz}, \mathrm{CDCl}_{3}\right)$ cis/trans ratio $=5: 4, \delta 7.74\left(\mathrm{~d}, J=8.4 \mathrm{~Hz}, 1.6 \mathrm{H}\right.$, Tos H-2,6 $\left.{ }^{t}\right), 7.66(\mathrm{~d}, J=$ $8.4 \mathrm{~Hz}, 2 \mathrm{H}$, Tos $\left.\mathrm{H}-2,6^{\mathrm{c}}\right), 7.39-7.20(\mathrm{~m}, 16.2 \mathrm{H}, \mathrm{Ar}-\mathrm{H})$, contained in this multiplet: $7.35(\mathrm{~d}, J=8.4 \mathrm{~Hz}, 1.6 \mathrm{H}$, Tos $\left.\mathrm{H}-3,5^{t}\right)$ and $7.32\left(\mathrm{~d}, J=8.4 \mathrm{~Hz}, 2 \mathrm{H}\right.$, Tos H-3, $\left.5^{c}\right), 7.14\left(\mathrm{dd}, J=8.1,1.2 \mathrm{~Hz}, 2 \mathrm{H}, \mathrm{Ph} \mathrm{H}-2,6^{c}\right), 7.04(\mathrm{dd}, J=$ 7.6, $\left.1.7 \mathrm{~Hz}, 1.6 \mathrm{H}, \mathrm{Ph} \mathrm{H}-2,6^{t}\right), 5.01\left(\mathrm{dd}, J=7.5,2.5 \mathrm{~Hz}, 0.8 \mathrm{H}, \mathrm{H}-2^{t}\right), 4.79\left(\mathrm{dd}, J=10.0,6.9 \mathrm{~Hz}, 1 \mathrm{H}, \mathrm{H}-2^{c}\right), 4.17$ ( ddd, $J=11.2,7.5,1.3 \mathrm{~Hz}, 1 \mathrm{H}, \mathrm{H}-5 \mathrm{a}^{c}$ ), $4.04\left(\mathrm{dd}, J=8.9,7.2 \mathrm{~Hz}, 0.8 \mathrm{H}, \mathrm{H}-5 \mathrm{a}^{t}\right), 3.53\left(\mathrm{t}, J=11.3 \mathrm{~Hz}, 1 \mathrm{H}, \mathrm{H}-5 \mathrm{~b}^{c}\right.$ ), 3.53-3.39 (m, 0.8H, H-4 $\left.{ }^{t}\right), 3.30\left(\mathrm{dd}, J=10.4,9.2 \mathrm{~Hz}, 0.8 \mathrm{H}, \mathrm{H}-5 \mathrm{~b}^{t}\right), 3.05-2.91\left(\mathrm{~m}, 1 \mathrm{H}, \mathrm{H}-4^{\mathrm{c}}\right), 2.74-2.63(\mathrm{~m}$,

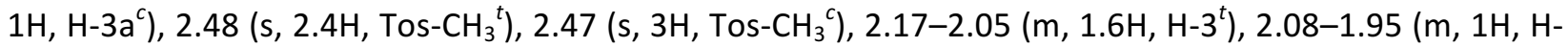
$\left.3 b^{c}\right)$.

${ }^{13} \mathrm{C}$ NMR $\left(75 \mathrm{MHz}, \mathrm{CDCl}_{3}\right) \delta 143.74,143.62,141.46,141.04,139.24,138.81,135.44,134.45,133.06$, $132.99,129.75,129.68,128.72,128.68,128.63,128.56,127.79,127.60,127.54,127.45,127.24,127.17$, $126.96,126.94,63.88,62.42,55.81,55.05,44.30,43.58,42.05,41.38,21.59,21.57$.

ESI MS found for $\left[\mathrm{M}^{+*}\right] \mathrm{m} / \mathrm{z} 411.1061$; calculated for $\left[\mathrm{M}^{+\cdot}\right] \mathrm{m} / \mathrm{z} 411.1060$.

\section{2-(4-chlorophenyl)-4-(perfluorophenyl)-1-tosylpyrrolidine (9b).}

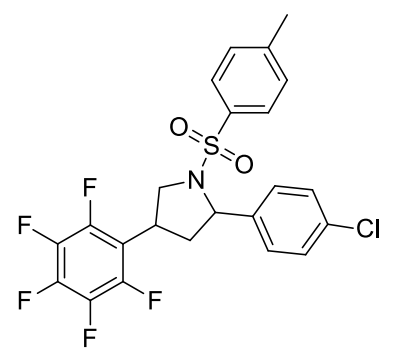

${ }^{1} \mathbf{H}$ NMR, COSY $\left(300 \mathrm{MHz}, \mathrm{CDCl}_{3}\right)$ cis/trans ratio $=7: 5, \delta 7.71\left(\mathrm{~d}, J=8.3 \mathrm{~Hz}, 1.4 \mathrm{H}\right.$, Tos H-2,6 $\left.6^{t}\right), 7.58(\mathrm{~d}, J=$ $8.2 \mathrm{~Hz}, 2 \mathrm{H}$, Tos $\left.\mathrm{H}-2,6^{c}\right), 7.34\left(\mathrm{~d}, J=8.9 \mathrm{~Hz}, 1.4 \mathrm{H}\right.$, Tos H-3, $\left.5^{t}\right), 7.32\left(\mathrm{~s}, 2.8 \mathrm{H}, 4-\mathrm{Cl}^{-} \mathrm{Ph}^{t}\right), 7.28(\mathrm{~d}, J=8.9 \mathrm{~Hz}, 2 \mathrm{H}$, Tos $\left.\mathrm{H}-3,5^{c}\right), 7.26\left(\mathrm{~s}, 4 \mathrm{H}, 4-\mathrm{Cl}_{-}-\mathrm{Ph}^{c}\right), 4.98\left(\mathrm{dd}, J=8.5,1.7 \mathrm{~Hz}, 0.7 \mathrm{H}, \mathrm{H}-2^{t}\right), 4.77\left(\mathrm{dd}, J=9.7,7.1 \mathrm{~Hz}, 1 \mathrm{H}, \mathrm{H}-2^{c}\right)$, 4.07 (ddd, $J=10.9,7.9,1.3 \mathrm{~Hz}, 1 \mathrm{H}, \mathrm{H}-5 \mathrm{a}^{c}$ ), $3.93\left(\mathrm{t}, J=8.4 \mathrm{~Hz}, 0.7 \mathrm{H}, \mathrm{H}-5 \mathrm{a}^{t}\right), 3.80-3.65\left(\mathrm{~m}, 0.7 \mathrm{H}, \mathrm{H}-4^{t}\right), 3.74$ $\left(\mathrm{t}, J=11.0 \mathrm{~Hz}, 1 \mathrm{H}, \mathrm{H}-5 \mathrm{~b}^{c}\right), 3.51\left(\mathrm{dd}, J=10.4,9.1 \mathrm{~Hz}, 0.7 \mathrm{H}, \mathrm{H}-5 \mathrm{~b}^{t}\right), 3.34-3.20\left(\mathrm{~m}, 1 \mathrm{H}, \mathrm{H}-4^{c}\right), 2.65-2.54(\mathrm{~m}$, $\left.1 \mathrm{H}, \mathrm{H}-3 \mathrm{a}^{\mathrm{c}}\right), 2.47\left(\mathrm{~s}, 2.1 \mathrm{H}, \operatorname{Tos}^{-\mathrm{CH}_{3}}{ }^{t}\right), 2.44\left(\mathrm{~s}, 3 \mathrm{H}, \mathrm{Tos}^{-\mathrm{CH}_{3}}{ }^{\mathrm{c}}\right), 2.44-2.33\left(\mathrm{~m}, 0.7 \mathrm{H}, \mathrm{H}-3 \mathrm{a}^{t}\right), 2.35-2.21(\mathrm{~m}, 1 \mathrm{H}$, $\mathrm{H}-3 b^{c}$ ), 2.04 (ddd, $J=12.2,6.3,2.4 \mathrm{~Hz}, 0.7 \mathrm{H}, \mathrm{H}-3 \mathrm{a}^{t}$ ). 
${ }^{19} \mathrm{~F}$ NMR $\left(282 \mathrm{MHz}, \mathrm{CDCl}_{3}\right) \delta-141.52--142.01(\mathrm{~m}, 2 \mathrm{~F}),-154.61--154.87(\mathrm{~m}, 1 \mathrm{~F}),-161.12--161.44(\mathrm{~m}$, 2F).

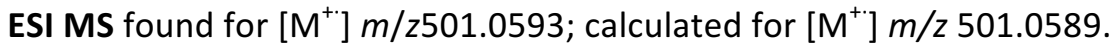

\section{2-(3-bromophenyl)-4-phenyl-1-tosylpyrrolidine (9c).}

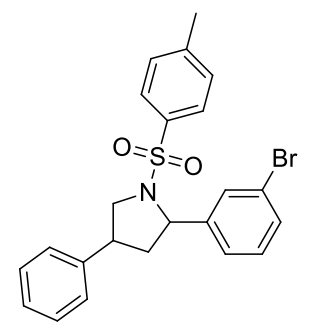

${ }^{1} \mathrm{H}$ NMR, COSY $\left(300 \mathrm{MHz}, \mathrm{CDCl}_{3}\right)$ cis/trans ratio $=6: 5, \delta 7.70\left(\mathrm{~d}, J=8.2 \mathrm{~Hz}, 1.7 \mathrm{H}\right.$, Tos H-2,6 $\left.6^{t}\right), 7.62(\mathrm{~d}, J=$ $8.3 \mathrm{~Hz}, 2 \mathrm{H}$, Tos $\left.\mathrm{H}-2,6^{c}\right), 7.47-7.16(\mathrm{~m}, 16.7 \mathrm{H}, \mathrm{Ar}-\mathrm{H})$, contained in this multiplet: $7.32(\mathrm{~d}, J=8.4 \mathrm{~Hz}, 1.7 \mathrm{H}$, Tos $\left.\mathrm{H}-3,5^{t}\right)$ and $7.28\left(\mathrm{~d}, J=8.4 \mathrm{~Hz}, 2 \mathrm{H}\right.$, Tos H-3, $\left.5^{c}\right), 7.12\left(\mathrm{dd}, J=7.7,1.6 \mathrm{~Hz}, 2 \mathrm{H}, \mathrm{Ph} \mathrm{H}-2,6^{c}\right), 7.04(\mathrm{dd}, J=$ 7.8, $\left.1.4 \mathrm{~Hz}, 1.7 \mathrm{H}, \mathrm{Ph} \mathrm{H}-2,6^{t}\right), 5.00\left(\mathrm{dd}, J=7.1,2.9 \mathrm{~Hz}, 0.85 \mathrm{H}, \mathrm{H}-2^{t}\right), 4.78\left(\mathrm{dd}, J=9.8,6.9 \mathrm{~Hz}, 1 \mathrm{H}, \mathrm{H}-2^{c}\right), 4.19$ (ddd, $J=11.0,7.5,1.2 \mathrm{~Hz}, 1 \mathrm{H}, \mathrm{H}-5 \mathrm{a}^{\mathrm{c}}$ ), 4.02 (dd, $J=8.9,7.1 \mathrm{~Hz}, 0.85 \mathrm{H}, \mathrm{H}-5 \mathrm{a}^{t}$ ), $3.50(\mathrm{t}, J=11.2 \mathrm{~Hz}, 1 \mathrm{H}, \mathrm{H}-$ $\left.5 b^{c}\right), 3.50-3.37\left(m, 0.85 \mathrm{H}, \mathrm{H}-4^{t}\right), 3.31\left(\mathrm{dd}, J=10.3,9.2 \mathrm{~Hz}, 0.85 \mathrm{H}, \mathrm{H}-5 \mathrm{~b}^{t}\right), 3.06-2.92\left(\mathrm{~m}, 1 \mathrm{H}, \mathrm{H}-4^{c}\right), 2.73-$ $2.62\left(\mathrm{~m}, 1 \mathrm{H}, \mathrm{H}-3 \mathrm{a}^{\mathrm{c}}\right), 2.45\left(\mathrm{~s}, 2.55 \mathrm{H}\right.$, Tos $\left.-\mathrm{CH}_{3}{ }^{t}\right), 2.44\left(\mathrm{~s}, 3 \mathrm{H}, \operatorname{Tos}^{-} \mathrm{CH}_{3}{ }^{\mathrm{c}}\right), 2.18-2.07\left(\mathrm{~m}, 1.7 \mathrm{H}, \mathrm{H}-3^{t}\right), 2.07-1.93$ $\left(\mathrm{m}, 1 \mathrm{H}, \mathrm{H}-3 \mathrm{~b}^{\mathrm{c}}\right)$.

${ }^{13} \mathrm{C} \mathrm{NMR}\left(75 \mathrm{MHz}, \mathrm{CDCl}_{3}\right) \delta 145.25,144.76,143.78,143.66,139.22,138.73,135.56,134.55,130.45$, 130.35, 130.07, 130.04, 129.77, 129.71, 129.37, 129.21, 128.73, 128.69, 127.57, 127.38, 127.27, 127.19, $127.00,126.97,125.25,124.92,122.68,122.59,63.88,62.42,55.78,55.03,44.33,43.66,42.05,41.36$, $21.60,21.58$.

ESI MS found for $\left[\mathrm{M}^{+}\right] \mathrm{m} / \mathrm{z} 455.0554$; calculated for $\left[\mathrm{M}^{+\cdot}\right] \mathrm{m} / \mathrm{z} 455.0555$.

\section{2-(4-methoxyphenyl)-4-phenyl-1-tosylpyrrolidine (9d).}

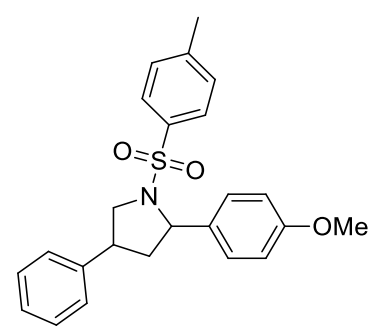

${ }^{1} \mathbf{H}$ NMR, COSY $\left(300 \mathrm{MHz}, \mathrm{CDCl}_{3}\right)$ cis/trans ratio $=11: 5,87.70\left(\mathrm{~d}, J=7.9 \mathrm{~Hz}, 0.9 \mathrm{H}\right.$, Tos H-2, $\left.6^{t}\right), 7.66(\mathrm{~d}, \mathrm{~J}=$ $7.9 \mathrm{~Hz}, 2 \mathrm{H}$, Tos $\left.\mathrm{H}-2,6^{c}\right), 7.38-7.21(\mathrm{~m}, 10.15 \mathrm{H}, \mathrm{Ar}-\mathrm{H})$, contained in this multiplet: $7.35(\mathrm{~d}, \mathrm{~J}=8.4 \mathrm{~Hz}, 0.9 \mathrm{H}$, 
4-MeOPh H-2, $6^{t}$ ), $7.34\left(\mathrm{~d}, J=7.9 \mathrm{~Hz}, 0.9 \mathrm{H}\right.$, Tos H-3,5 $\left.{ }^{t}\right), 7.30\left(\mathrm{~d}, J=8.3 \mathrm{~Hz}, 2 \mathrm{H}, 4-\mathrm{MeOPh} \mathrm{H}-2,6^{c}\right)$ and 7.30 (d, $\left.J=7.9 \mathrm{~Hz}, 2 \mathrm{H}, \operatorname{Tos} \mathrm{H}-3,5^{c}\right), 7.16\left(\mathrm{~d}, J=7.4 \mathrm{~Hz}, 2 \mathrm{H}, \mathrm{Ph} \mathrm{H}-2,6^{c}\right.$ ), 7.06 (d, J = 7.2 Hz, 0.9H, Ph H-2, $\left.6^{t}\right), 6.92$ (d, $\left.J=8.4 \mathrm{~Hz}, 0.9 \mathrm{H}, 4-\mathrm{MeOPh} \mathrm{H}-3,5^{t}\right), 6.87\left(\mathrm{~d}, J=8.3 \mathrm{~Hz}, 2 \mathrm{H}, 4-\mathrm{MeOPh} \mathrm{H}-3,5^{c}\right.$ ), $5.04(\mathrm{~d}, J=8.0 \mathrm{~Hz}, 0.45 \mathrm{H}$, $\left.\mathrm{H}-2^{t}\right), 4.80\left(\mathrm{dd}, J=9.3,7.1 \mathrm{~Hz}, 1 \mathrm{H}, \mathrm{H}-2^{c}\right.$ ) , $4.18\left(\mathrm{dd}, J=10.7,7.9 \mathrm{~Hz}, 1 \mathrm{H}, \mathrm{H}-5 \mathrm{a}^{c}\right), 4.04(\mathrm{t}, J=8.3 \mathrm{~Hz}, 0.45 \mathrm{H}, \mathrm{H}-$ $\left.5 a^{t}\right), 3.84\left(\mathrm{~s}, 1.35 \mathrm{H}, \mathrm{OCH}_{3}{ }^{t}\right), 3.83\left(\mathrm{~s}, 3 \mathrm{H}, \mathrm{OCH}_{3}{ }^{\mathrm{c}}\right), 3.56-3.43\left(\mathrm{~m}, 0.45 \mathrm{H}, \mathrm{H}-4^{t}\right), 3.54\left(\mathrm{t}, J=11.3 \mathrm{~Hz}, 1 \mathrm{H}, \mathrm{H}-5 \mathrm{~b}^{c}\right)$, $3.31\left(\mathrm{t}, J=9.8 \mathrm{~Hz}, 0.45 \mathrm{H}, \mathrm{H}-5 \mathrm{~b}^{t}\right), 3.05-2.92\left(\mathrm{~m}, 1 \mathrm{H}, \mathrm{H}-4^{c}\right), 2.73-2.62\left(\mathrm{~m}, 1 \mathrm{H}, \mathrm{H}-3 \mathrm{a}^{\mathrm{c}}\right), 2.48(\mathrm{~s}, 1.35 \mathrm{H}$, Tos$\left.\mathrm{CH}_{3}{ }^{t}\right), 2.46\left(\mathrm{~s}, 3 \mathrm{H}\right.$, Tos $\left.-\mathrm{CH}_{3}{ }^{c}\right), 2.23-2.05\left(\mathrm{~m}, 0.9 \mathrm{H}, \mathrm{H}-3^{t}\right), 2.14-2.02\left(\mathrm{~m}, 1 \mathrm{H}, \mathrm{H}-3 \mathrm{~b}^{\mathrm{c}}\right)$.

${ }^{13} \mathrm{C}$ NMR $(101 \mathrm{MHz}, \mathrm{CDCl} 3) \delta 158.98,158.84,143.58,143.37,139.77,139.28,135.87,135.09,134.81$, 134.57, 129.75, 129.66, 128.76, 128.71, 127.79, 127.68, 127.52, 127.34, 127.21, 127.12, 127.10, 127.07, $113.94,113.88,64.18,62.67,55.88,55.42,55.40,55.14,44.45,43.60,42.24,41.55,21.66,21.63$.

ESI MS found for $\left[\mathrm{M}^{+}\right] \mathrm{m} / \mathrm{z} 407.1554$; calculated for $\left[\mathrm{M}^{++}\right] \mathrm{m} / \mathrm{z} 407.1555$.

\section{2-(4-(tert-butyl)phenyl)-4-phenyl-1-tosylpyrrolidine (9e).}

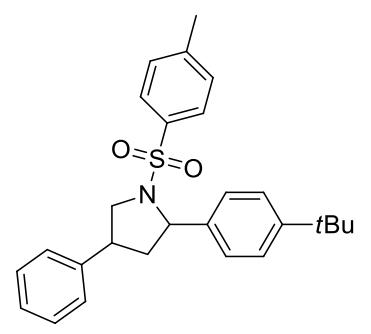

${ }^{1} \mathbf{H}$ NMR, CosY $\left(300 \mathrm{MHz}, \mathrm{CDCl}_{3}\right)$ cis/trans ratio $=3: 2, \delta 7.75\left(\mathrm{~d}, J=7.8 \mathrm{~Hz}, 1.33 \mathrm{H}\right.$, Tos $\left.\mathrm{H}-2,6^{t}\right), 7.63(\mathrm{~d}, J=$ $7.9 \mathrm{~Hz}, 2 \mathrm{H}$, Tos $\left.\mathrm{H}-2,6^{c}\right), 7.43-7.23(\mathrm{~m}, 11.7 \mathrm{H}, \mathrm{Ar}-\mathrm{H})$, contained in this multiplet: $7.33(\mathrm{~d}, J=7.8 \mathrm{~Hz}, 1.33 \mathrm{H}$, Tos $\left.\mathrm{H}-3,5^{t}\right)$ and $7.27\left(\mathrm{~d}, J=7.9 \mathrm{~Hz}, 2 \mathrm{H}\right.$, Tos $\left.\mathrm{H}-3,5^{c}\right), 7.17\left(\mathrm{~d}, J=7.4 \mathrm{~Hz}, 2 \mathrm{H}, \mathrm{Ph} \mathrm{H}-2,6^{c}\right), 7.07(\mathrm{~d}, J=7.8 \mathrm{~Hz}$, 1.33H, Ph H-2, $\left.6^{t}\right), 5.08\left(\mathrm{~d}, J=8.2 \mathrm{~Hz}, 0.66 \mathrm{H}, \mathrm{H}-2^{t}\right), 4.87\left(\mathrm{dd}, J=9.5,7.2 \mathrm{~Hz}, 1 \mathrm{H}, \mathrm{H}-2^{c}\right), 4.24(\mathrm{dd}, J=10.9,7.6$ $\left.\mathrm{Hz}, 1 \mathrm{H}, \mathrm{H}-5 \mathrm{a}^{c}\right), 4.07\left(\mathrm{t}, J=8.3 \mathrm{~Hz}, 0.66 \mathrm{H}, \mathrm{H}-5 \mathrm{a}^{t}\right), 3.62-3.47\left(\mathrm{~m}, 0.66 \mathrm{H}, \mathrm{H}-4^{t}\right), 3.55\left(\mathrm{t}, J=11.2 \mathrm{~Hz}, 1 \mathrm{H}, \mathrm{H}-5 \mathrm{~b}^{c}\right)$, $3.35\left(\mathrm{t}, J=9.8 \mathrm{~Hz}, 0.66 \mathrm{H}, \mathrm{H}-5 \mathrm{~b}^{t}\right), 3.11-2.96\left(\mathrm{~m}, 1 \mathrm{H}, \mathrm{H}-4^{\mathrm{c}}\right), 2.78-2.66\left(\mathrm{~m}, 1 \mathrm{H}, \mathrm{H}-3 \mathrm{a}^{\mathrm{c}}\right), 2.48\left(\mathrm{~s}, 2 \mathrm{H}, \mathrm{Tos}^{-\mathrm{CH}_{3}}{ }^{t}\right)$, $2.45\left(\mathrm{~s}, 3 \mathrm{H}, \operatorname{Tos}-\mathrm{CH}_{3}{ }^{\mathrm{c}}\right), 2.27-2.04\left(\mathrm{~m}, 1.22 \mathrm{H}, \mathrm{H}-3^{t}\right), 2.18-2.05\left(\mathrm{~m}, 1 \mathrm{H}, \mathrm{H}-3 \mathrm{~b}^{\mathrm{c}}\right), 1.37\left(\mathrm{~s}, 6 \mathrm{H}, t \mathrm{Bu}{ }^{t}\right), 1.36(\mathrm{~s}, 9 \mathrm{H}$, $\left.t \mathrm{Bu}^{\mathrm{C}}\right)$.

${ }^{13} \mathrm{C}$ NMR $\left(101 \mathrm{MHz}, \mathrm{CDCl}_{3}\right) \delta 150.26,150.06,143.54,143.27,139.88,139.83,139.37,139.32,136.12$, $134.98,129.77,129.63,128.80,128.75,127.73,127.55,127.25,127.18,127.15,127.14,126.38,125.97$, 125.44, 125.43, 64.28, 62.97, 55.98, 55.21, 44.35, 43.77, 42.12, 41.62, 34.61, 34.60, 31.55 (two coincident resonances), 21.70, 21.67.

ESI MS found for $\left[\mathrm{M}^{+\cdot}\right] \mathrm{m} / \mathrm{z} 433.2058$; calculated for $\left[\mathrm{M}^{++}\right] \mathrm{m} / \mathrm{z} 433.2076$. 
2-(4-chlorophenyl)-4-phenyl-1-((4-(trifluoromethyl)phenyl)sulfonyl)pyrrolidine (9f).

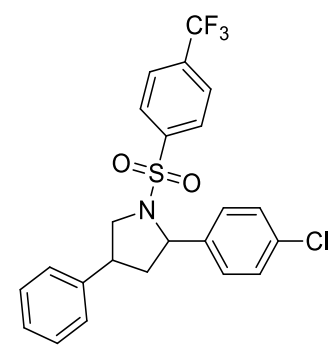

${ }^{1} \mathrm{H}$ NMR, COSY $\left(300 \mathrm{MHz}, \mathrm{CDCl}_{3}\right)$ cis/trans ratio $=7: 5, \delta 7.92\left(\mathrm{~d}, J=8.2 \mathrm{~Hz}, 1.4 \mathrm{H}, 4-\mathrm{CF}_{3} \mathrm{C}_{4} \mathrm{H}_{6} \mathrm{H}-3,5^{t}\right), 7.82-$ $7.76(\mathrm{~m}, 3.4 \mathrm{H})$ contained in this multiplet: $7.79\left(\mathrm{~d}, J=8.2 \mathrm{~Hz}, 1.4 \mathrm{H}, 4-\mathrm{CF}_{3} \mathrm{C}_{4} \mathrm{H}_{6} \mathrm{H}-2,6^{t}\right)$ and $7.78(\mathrm{~d}, J=8.4$ $\left.\mathrm{Hz}, 2 \mathrm{H}, 4-\mathrm{CF}_{3} \mathrm{C}_{4} \mathrm{H}_{6} \mathrm{H}-3,5^{c}\right), 7.72\left(\mathrm{~d}, J=8.4 \mathrm{~Hz}, 2 \mathrm{H}, 4-\mathrm{CF}_{3} \mathrm{C}_{4} \mathrm{H}_{6} \mathrm{H}-2,6^{c}\right), 7.40-7.06(\mathrm{~m}, 15.4 \mathrm{H}, \mathrm{Ar}-\mathrm{H}), 5.07$ (dd, $J$ $\left.=7.9,2.2 \mathrm{~Hz}, 0.7 \mathrm{H}, \mathrm{H}-2^{t}\right), 4.89\left(\mathrm{dd}, J=9.9,6.9 \mathrm{~Hz}, 1 \mathrm{H}, \mathrm{H}-2^{c}\right), 4.29\left(\mathrm{dd}, J=10.4,7.9,1 \mathrm{H}, \mathrm{H}-5 \mathrm{a}^{c}\right), 4.10(\mathrm{dd}, J=$ 9.1, 7.3 Hz, 0.7H, H-5a $\left.{ }^{t}\right), 3.56\left(\mathrm{t}, J=11.0 \mathrm{~Hz}, 1 \mathrm{H}, \mathrm{H}-5 \mathrm{~b}^{c}\right), 3.58-3.47\left(\mathrm{~m}, 0.7 \mathrm{H}, \mathrm{H}-4^{t}\right), 3.41(\mathrm{t}, J=9.6 \mathrm{~Hz}, 0.7 \mathrm{H}$, $\left.\mathrm{H}-5 \mathrm{~b}^{t}\right), 3.27-3.13\left(\mathrm{~m}, 1 \mathrm{H}, \mathrm{H}-4^{c}\right), 2.84-2.72\left(\mathrm{~m}, 1 \mathrm{H}, \mathrm{H}-3 \mathrm{a}^{c}\right), 2.34-2.16\left(\mathrm{~m}, 1.4 \mathrm{H}, \mathrm{H}-3^{t}\right), 2.14-2.04(\mathrm{~m}, 1 \mathrm{H}, \mathrm{H}-$ $\left.3 b^{c}\right)$.

${ }^{13} \mathrm{C}$ NMR $\left(101 \mathrm{MHz}, \mathrm{CDCl}_{3}\right.$ ) $\delta 142.82,141.51,140.97,140.16,139.15,138.58,134.59$ (q, J = 33.2 Hz), 134.33 (q, $J=32.9 \mathrm{~Hz}$ ), 133.60, 133.42, 128.95, 128.93, 128.80 (two coincident resonances), 128.26, 128.03, 127.74, $127.68,127.55,127.48,127.11,126.99,126.36$ (q, $J=3.7 \mathrm{~Hz}$ ), 126.18 (q, $J=3.7 \mathrm{~Hz}$ ), 123.37 (q, $J=273.0 \mathrm{~Hz}$, two coincident resonances), 64.15, 62.85, 55.82, 55.33, 44.38, 43.86, 42.25, 41.54.

${ }^{19}$ F NMR $\left(376 \mathrm{MHz}, \mathrm{CDCl}_{3}\right) \delta-63.02,-63.03$.

ESI MS found for $\left[\mathrm{M}^{++}\right] \mathrm{m} / \mathrm{z465.0770;}$; calculated for $\left[\mathrm{M}^{+\cdot}\right] \mathrm{m} / \mathrm{z} 465.0777$.

\section{4-(4-chlorophenyl)-1-((4-nitrophenyl)sulfonyl)-2-phenylpyrrolidine (9g).}

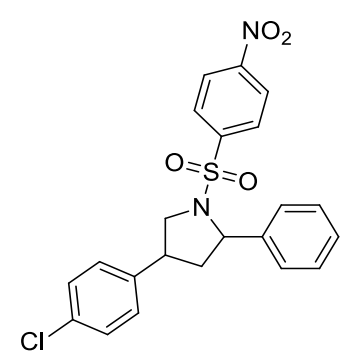

${ }^{1} \mathrm{H}$ NMR, CosY $\left(300 \mathrm{MHz}, \mathrm{CDCl}_{3}\right)$ cis/trans ratio $=1: 5,88.28\left(\mathrm{~d}, J=9.0 \mathrm{~Hz}, 2 \mathrm{H}, 4-\mathrm{NO}_{2} \mathrm{C}_{4} \mathrm{H}_{6} \mathrm{H}-3,5^{t}\right), 8.18$ (d, J $\left.=9.1 \mathrm{~Hz}, 0.4 \mathrm{H}, 4-\mathrm{NO}_{2} \mathrm{C}_{4} \mathrm{H}_{6} \mathrm{H}-3,5^{c}\right) 7.87\left(\mathrm{~d}, J=9.0 \mathrm{~Hz}, 2 \mathrm{H}, 4-\mathrm{NO}_{2} \mathrm{C}_{4} \mathrm{H}_{6} \mathrm{H}-2,6^{t}\right), 7.65(\mathrm{~d}, J=9.1 \mathrm{~Hz}, 0.4 \mathrm{H}, 4-$ $\left.\mathrm{NO}_{2} \mathrm{C}_{4} \mathrm{H}_{6} \mathrm{H}-2,6^{\mathrm{c}}\right), 7.36-7.20(\mathrm{~m}, 8.4 \mathrm{H}, \mathrm{Ar}-\mathrm{H})$, contained in this multiplet: $7.31\left(\mathrm{~d}, \mathrm{~J}=8.3 \mathrm{~Hz}, 0.4 \mathrm{H}, 4-\mathrm{ClC}_{4} \mathrm{H}_{6}\right.$ $\left.\mathrm{H}-3,5^{c}\right)$ and $7.28\left(\mathrm{~d}, J=8.4 \mathrm{~Hz}, 2 \mathrm{H}, 4-\mathrm{ClC}_{4} \mathrm{H}_{6} \mathrm{H}-3,5^{t}\right), 7.16\left(\mathrm{~d}, J=8.3 \mathrm{~Hz}, 0.4 \mathrm{H}, 4-\mathrm{ClC}_{4} \mathrm{H}_{6} \mathrm{H}-2,6^{c}\right)$ and $7.08(\mathrm{~d}, J$ $\left.=8.4 \mathrm{~Hz}, 2 \mathrm{H}, 4-\mathrm{ClC}_{4} \mathrm{H}_{6} \mathrm{H}-2,6^{t}\right), 5.14\left(\mathrm{dd}, J=7.6,3.1 \mathrm{~Hz}, 1 \mathrm{H}, \mathrm{H}-2^{t}\right), 4.99\left(\mathrm{dd}, J=10.0,6.9 \mathrm{~Hz}, 0.2 \mathrm{H}, \mathrm{H}-2^{c}\right.$ ), $4.36\left(\mathrm{ddd}, J=10.2,7.2,1.4 \mathrm{~Hz}, 0.2 \mathrm{H}, \mathrm{H}-5 \mathrm{a}^{c}\right), 4.06\left(\mathrm{dd}, J=8.9,6.8 \mathrm{~Hz}, 1 \mathrm{H}, \mathrm{H}-5 \mathrm{a}^{t}\right), 3.63-3.49\left(\mathrm{~m}, 1 \mathrm{H}, \mathrm{H}-4^{t}\right)$, 
$3.50\left(\mathrm{t}, J=9.1 \mathrm{~Hz}, 1 \mathrm{H}, \mathrm{H}-5 \mathrm{~b}^{t}\right), 3.49\left(\mathrm{t}, J=10.2 \mathrm{~Hz}, 0.2 \mathrm{H}, \mathrm{H}-5 \mathrm{~b}^{\mathrm{c}}\right), 3.40-3.37\left(\mathrm{~m}, 0.2 \mathrm{H}, \mathrm{H}-4^{\mathrm{c}}\right), 2.86-2.76(\mathrm{~m}$, $\left.0.2 \mathrm{H}, \mathrm{H}-3 \mathrm{a}^{\mathrm{c}}\right), 2.37-2.20\left(\mathrm{~m}, 2 \mathrm{H}, \mathrm{H}-3^{t}\right), 2.21-2.06\left(\mathrm{~m}, 0.2 \mathrm{H}, \mathrm{H}-3 \mathrm{~b}^{c}\right)$.

${ }^{13} \mathrm{C}$ NMR $\left(75 \mathrm{MHz}, \mathrm{CDCl}_{3}\right) \delta 149.96,144.22,141.54,137.75,133.16,128.97,128.61,128.44,128.27$, $127.73,126.24,124.15,63.35,54.98,42.38,41.07$.

ESI MS found for $\left[\mathrm{M}^{+\cdot}\right] \mathrm{m} / \mathrm{z} 442.0747$; calculated for $\left[\mathrm{M}^{++}\right] \mathrm{m} / \mathrm{z} 442.0754$.

\section{2-(4-methoxyphenyl)-3-methyl-4-phenyl-1-tosylpyrrolidine (9h).}

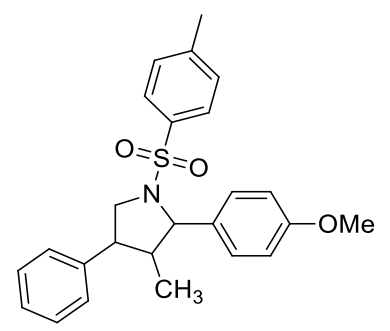

${ }^{1} \mathbf{H}$ NMR, CosY $\left(300 \mathrm{MHz}, \mathrm{CDCl}_{3}\right)$ cis/trans ratio $=1: 1, \delta 7.71\left(\mathrm{~d}, J=8.3 \mathrm{~Hz}, 2 \mathrm{H}\right.$, Tos $\left.\mathrm{H}-2,6^{t}\right), 7.59(\mathrm{~d}, J=8.3$ $\mathrm{Hz}, 2 \mathrm{H}$, Tos $\left.\mathrm{H}-2,6^{c}\right), 7.34-7.22(\mathrm{~m}, 14 \mathrm{H}, \mathrm{Ar}-\mathrm{H})$, contained in this multiplet: $7.31(\mathrm{~d}, J=8.3 \mathrm{~Hz}, 2 \mathrm{H}$, Tos $\mathrm{H}$ $\left.3,5^{t}\right), 7.29\left(\mathrm{~d}, J=8.3 \mathrm{~Hz}, 2 \mathrm{H}, \mathrm{Tos} \mathrm{H}-3,5^{c}\right)$, and $7.28\left(\mathrm{~d}, J=8.7 \mathrm{~Hz}, 4 \mathrm{H}, 4-\mathrm{MeOPh} \mathrm{H}-2,6^{c, t}\right) 7.17$ (dd, J = 8.1, 1.3Hz, $2 \mathrm{H}, \mathrm{Ph} \mathrm{H}-2,6^{c}$ ), 7.00 (dd, $J=7.6,1.5 \mathrm{~Hz}, 2 \mathrm{H}, \mathrm{Ph} \mathrm{H}-2,6^{t}$ ), 6.88 (d, $J=8.7 \mathrm{~Hz}, 2 \mathrm{H}, 4-\mathrm{MeOPh} \mathrm{H}-2,6^{c}$ ), $6.86\left(\mathrm{~d}, J=8.7 \mathrm{~Hz}, 2 \mathrm{H}, 4-\mathrm{MeOPh} \mathrm{H}-2,6^{t}\right), 4.51\left(\mathrm{~d}, J=3.4 \mathrm{~Hz}, 1 \mathrm{H}, \mathrm{H}-2^{t}\right), 4.17\left(\mathrm{~d}, J=9.3,1 \mathrm{H}, \mathrm{H}-2^{c}\right), 4.12(\mathrm{dd}, J$ $\left.=10.8,7.4 \mathrm{~Hz}, 1 \mathrm{H}, \mathrm{H}-5 \mathrm{a}^{c}\right), 4.02\left(\mathrm{dd}, J=9.3,7.2 \mathrm{~Hz}, 1 \mathrm{H}, \mathrm{H}-5 \mathrm{a}^{t}\right), 3.84\left(\mathrm{~s}, 6 \mathrm{H}, \mathrm{OCH}_{3}^{c, t}\right), 3.76(\mathrm{t}, J=9.1 \mathrm{~Hz}, 1 \mathrm{H}$, $\left.\mathrm{H}-5 \mathrm{~b}^{t}\right), 3.64-3.57\left(\mathrm{~m}, 1 \mathrm{H}, \mathrm{H}-3^{t}\right), 3.60\left(\mathrm{t}, J=11.2 \mathrm{~Hz}, 1 \mathrm{H}, \mathrm{H}-5 \mathrm{~b}^{\mathrm{c}}\right), 2.62-2.48\left(\mathrm{~m}, 1 \mathrm{H}, \mathrm{H}-4^{\mathrm{c}}\right), 2.46(\mathrm{~s}, 6 \mathrm{H}$, Tos$\left.\mathrm{CH}_{3}{ }^{c, t}\right), 2.46-2.34\left(\mathrm{~m}, 2 \mathrm{H}, \mathrm{H}-3^{t}\right), 2.31-2.16\left(\mathrm{~m}, 1 \mathrm{H}, \mathrm{H}-3^{\mathrm{c}}\right), 0.82\left(\mathrm{~d}, J=6.5 \mathrm{~Hz}, 3 \mathrm{H}, 2-\mathrm{CH}_{3}{ }^{c}\right), 0.39(\mathrm{~d}, J=7.1 \mathrm{~Hz}$, $\left.3 \mathrm{H}, 2-\mathrm{CH}_{3}{ }^{t}\right)$.

${ }^{13} \mathrm{C}$ NMR $\left(101 \mathrm{MHz}, \mathrm{CDCl}_{3}\right) \delta 159.15,158.94,143.40,143.29,138.46,138.45,135.95,135.33,134.39$, 133.45, 129.63, 129.57, 128.87, 128.51, 128.32, 127.92, 127.66, 127.65, 127.59, 127.57, 127.43, 126.82, 113.91, 113.83, 71.34, 69.91, 56.09, 55.44, 55.41, 51.63, 51.45, 50.93, 47.67, 44.42, 21.67 (two coincident resonances), 14.44, 13.86.

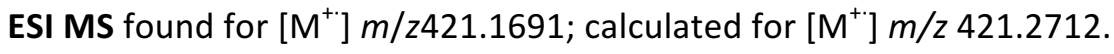


2-(4-benzhydrylphenyl)-4-phenyl-1-tosylpyrrolidine (9i).

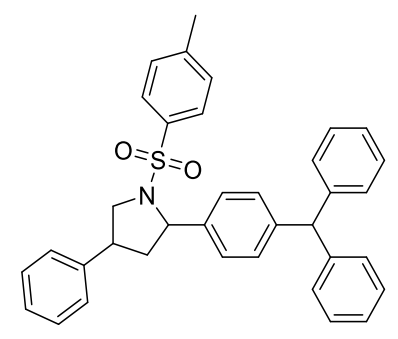

${ }^{1} \mathrm{H}$ NMR, COSY $\left(300 \mathrm{MHz}, \mathrm{CDCl}_{3}\right)$ cis/trans ratio $=5: 3, \delta 7.74\left(\mathrm{~d}, J=7.8 \mathrm{~Hz}, 1.2 \mathrm{H}, \mathrm{Tos} \mathrm{H}-2,6^{t}\right), 7.63(\mathrm{~d}, J=$ $7.8 \mathrm{~Hz}, 2 \mathrm{H}$, Tos $\left.\mathrm{H}-2,6^{\mathrm{c}}\right), 7.39-7.04(\mathrm{~m}, 33.6 \mathrm{H}, \mathrm{Ar}-\mathrm{H})$, contained in this multiplet: $7.32(\mathrm{~d}, J=7.8 \mathrm{~Hz}, 1.2 \mathrm{H}$, Tos $\left.\mathrm{H}-3,5^{t}\right)$ and $7.25\left(\mathrm{~d}, J=7.8 \mathrm{~Hz}, 2 \mathrm{H}\right.$, Tos $\left.\mathrm{H}-3,5^{c}\right), 5.58\left(\mathrm{~s}, 0.6 \mathrm{H}\right.$, benzhydryl $\left.\mathrm{CH}^{t}\right), 5.56(\mathrm{~s}, 1 \mathrm{H}$, benzhydryl $\left.\mathrm{CH}^{c}\right), 5.08\left(\mathrm{~d}, J=8.0 \mathrm{~Hz}, 0.6 \mathrm{H}, \mathrm{H}-2^{t}\right), 4.87\left(\mathrm{dd}, J=9.3,7.5 \mathrm{~Hz}, 1 \mathrm{H}, \mathrm{H}-2^{c}\right), 4.21(\mathrm{dd}, J=11.1,7.5 \mathrm{~Hz}, 1 \mathrm{H}, \mathrm{H}-$ $\left.5 \mathrm{a}^{\mathrm{c}}\right), 4.04\left(\mathrm{t}, J=8.2 \mathrm{~Hz}, 0.6 \mathrm{H}, \mathrm{H}-5 \mathrm{a}^{\mathrm{t}}\right), 3.51\left(\mathrm{t}, J=11.2 \mathrm{~Hz}, 1 \mathrm{H}, \mathrm{H}-5 \mathrm{~b}^{\mathrm{c}}\right), 3.54-3.43\left(\mathrm{~m}, 0.6 \mathrm{H}, \mathrm{H}-4^{t}\right), 3.32(\mathrm{t}, J=$ $\left.9.8 \mathrm{~Hz}, 0.6 \mathrm{H}, \mathrm{H}-5 \mathrm{~b}^{t}\right), 3.08-2.91\left(\mathrm{~m}, 1 \mathrm{H}, \mathrm{H}-4^{c}\right), 2.78-2.63\left(\mathrm{~m}, 1 \mathrm{H}, \mathrm{H}-3 \mathrm{a}^{\mathrm{c}}\right), 2.46\left(\mathrm{~s}, 1.8 \mathrm{H}, \mathrm{Tos}^{\mathrm{C}} \mathrm{CH}_{3}{ }^{t}\right), 2.43(\mathrm{~s}$, $3 \mathrm{H}$, Tos $\left.-\mathrm{CH}_{3}{ }^{c}\right), 2.25-2.05\left(\mathrm{~m}, 1.2 \mathrm{H}, \mathrm{H}-3^{t}\right), 2.15-2.01\left(\mathrm{~m}, 1 \mathrm{H}, \mathrm{H}-3 \mathrm{~b}^{c}\right)$.

${ }^{13} \mathrm{C} \mathrm{NMR}\left(75 \mathrm{MHz}, \mathrm{CDCl}_{3}\right) \delta 143.95,143.91,143.53,143.32,142.94,142.76,140.79,140.47,139.62$, $139.11,135.88,134.78,129.69,129.58,129.49,129.46,128.69,128.66,128.36,127.60,127.41,127.17$, $127.06,127.02,126.50,126.37,126.13,64.18,62.87,56.62,56.58,55.82,55.05,44.32,43.71,41.95$, $41.46,21.60,21.57$.

ESI MS found for $\left[\mathrm{M}^{\left.+{ }^{+}\right]} \mathrm{m} / \mathrm{z}\right.$ 543.2221; calculated for $\left[\mathrm{M}^{++}\right] \mathrm{m} / \mathrm{z} 543.2232$. 
Table S5. [3+2] Cycloaddition of Aziridines and Alkenes

\begin{tabular}{|c|c|c|c|c|c|c|c|c|c|}
\hline \multirow[b]{2}{*}{ entry $^{a}$} & \multicolumn{2}{|c|}{$\underbrace{R_{2}}_{=S=0}$} & $\begin{array}{r}\mathrm{R}_{4} \\
8\end{array}$ & \multicolumn{2}{|c|}{$\begin{array}{c}\begin{array}{c}\text { Mn-porph } \\
(1 \mathrm{~mol} \%)\end{array} \\
\underset{\mathrm{DCE}}{100^{\circ} \mathrm{C}}\end{array}$} & $\mathrm{R}_{2}$ & & & \\
\hline & catalyst & $\mathrm{R}_{1}$ & $\mathrm{R}_{2}$ & $\mathrm{R}_{3}$ & $\mathrm{R}_{4}$ & product & cis/trans & $t(h)$ & yield (\%) \\
\hline 1 & UNLPF-13-Mn ${ }^{\mathrm{II}} \mathrm{Cl}$ & $\mathrm{Ph}$ & $\mathrm{CH}_{3}$ & 4-Cl-Ph & $\mathrm{H}$ & $9 a$ & n.a. & 24 & n.r. \\
\hline 2 & UNLPF-14-Mn"II & $\mathrm{Ph}$ & $\mathrm{CH}_{3}$ & 4-Cl-Ph & $\mathrm{H}$ & $9 a$ & $5: 4$ & 8 & 95 \\
\hline 3 & PCN-223-Mn'"I Cl & $\mathrm{Ph}$ & $\mathrm{CH}_{3}$ & 4-Cl-Ph & $\mathrm{H}$ & $9 a$ & n.a. & 24 & n.r. \\
\hline 4 & $\mathrm{PCN}-223-\mathrm{Mn}^{\prime \prime \prime}\left[\mathrm{SbF}_{6}\right]$ & $\mathrm{Ph}$ & $\mathrm{CH}_{3}$ & 4-Cl-Ph & $\mathrm{H}$ & $9 a$ & $6: 5$ & 24 & $60^{b}$ \\
\hline 5 & $\mathrm{MnTPP}\left[\mathrm{SbF}_{6}\right]$ & $\mathrm{Ph}$ & $\mathrm{CH}_{3}$ & 4-Cl-Ph & $\mathrm{H}$ & $9 a$ & $10: 9$ & 12 & $63^{b, c}$ \\
\hline 6 & $\mathrm{MnTPP}\left[\mathrm{BF}_{4}\right]$ & $\mathrm{Ph}$ & $\mathrm{CH}_{3}$ & 4-Cl-Ph & $\mathrm{H}$ & $9 a$ & - & 12 & $57^{b, c}$ \\
\hline 7 & $\mathrm{MnTPP}\left[\mathrm{SbF}_{6}\right]$ & $\mathrm{Ph}$ & $\mathrm{CH}_{3}$ & $4-\mathrm{Cl}-\mathrm{Ph}$ & $\mathrm{H}$ & $9 a$ & $10: 9$ & 12 & $13^{c}$ \\
\hline 8 & 2c-Mn & $\mathrm{Ph}$ & $\mathrm{CH}_{3}$ & 4-Cl-Ph & $\mathrm{H}$ & $9 a$ & $6: 5$ & 12 & $54^{b, c}$ \\
\hline 9 & UNLPF-14-Mn"'I & $\mathrm{C}_{6} \mathrm{~F}_{5}$ & $\mathrm{CH}_{3}$ & 4-Cl-Ph & $\mathrm{H}$ & $9 b$ & $7: 5$ & 10 & 88 \\
\hline 10 & UNLPF-14-Mn"II & $\mathrm{Ph}$ & $\mathrm{CH}_{3}$ & $3-\mathrm{Br}-\mathrm{Ph}$ & $\mathrm{H}$ & $9 c$ & $6: 5$ & 8 & 93 \\
\hline 11 & UNLPF-14-Mn"II & $\mathrm{Ph}$ & $\mathrm{CH}_{3}$ & 4-MeO-Ph & $\mathrm{H}$ & $9 d$ & $11: 5$ & 8 & 95 \\
\hline 12 & UNLPF-14-Mn"II & $\mathrm{Ph}$ & $\mathrm{CH}_{3}$ & 4-tBu-Ph & $\mathrm{H}$ & $9 e$ & $3: 2$ & 8 & 91 \\
\hline 13 & UNLPF-14-Mn"II & $\mathrm{Ph}$ & $\mathrm{CF}_{3}$ & 4-Cl-Ph & $\mathrm{H}$ & gf & $7: 5$ & 18 & 96 \\
\hline 14 & $\operatorname{MnTPP}\left[\mathrm{SbF}_{6}\right]$ & $\mathrm{Ph}$ & $\mathrm{CF}_{3}$ & 4-Cl-Ph & $\mathrm{H}$ & $9 f$ & $1: 1$ & 24 & $35^{b, c}$ \\
\hline 15 & UNLPF-14-Mn"II & 4-Cl-Ph & $\mathrm{NO}_{2}$ & $\mathrm{Ph}$ & $\mathrm{H}$ & $9 g$ & $1: 5$ & 24 & 93 \\
\hline 16 & UNLPF-14-Mn"II & $\mathrm{Ph}$ & $\mathrm{CH}_{3}$ & 4-MeO-Ph & $\mathrm{CH}_{3}$ & $9 \mathrm{~h}$ & $1: 1$ & 12 & 87 \\
\hline 17 & $\mathrm{MnTPP}\left[\mathrm{SbF}_{6}\right]$ & $\mathrm{Ph}$ & $\mathrm{CH}_{3}$ & 4-MeO-Ph & $\mathrm{CH}_{3}$ & $9 \mathrm{~h}$ & $1: 1$ & 24 & $19^{b, c}$ \\
\hline 18 & UNLPF-14-Mn"II & $\mathrm{Ph}$ & $\mathrm{CH}_{3}$ & $\mathrm{PhCHPh}_{2}$ & $\mathrm{H}$ & $9 i$ & $5: 3$ & 24 & 10 \\
\hline 19 & UNLPF-14-Mn"II & $\mathrm{Ph}$ & $\mathrm{CH}_{3}$ & $\mathrm{PhCHPh}_{2}$ & $\mathrm{H}$ & $9 i$ & $5: 3$ & 24 & $24^{b}$ \\
\hline 20 & $\operatorname{MnTPP}\left[\mathrm{SbF}_{6}\right]$ & $\mathrm{Ph}$ & $\mathrm{CH}_{3}$ & $\mathrm{PhCHPh}_{2}$ & $\mathrm{H}$ & $9 i$ & $5: 3$ & 24 & $67^{b}$ \\
\hline 21 & UNLPF-14-Mn"II & $\mathrm{Ph}$ & $\mathrm{CH}_{3}$ & $4-\mathrm{Cl}-\mathrm{Ph}$ & $\mathrm{H}$ & $9 a$ & $5: 4$ & 10 & $92^{d}$ \\
\hline
\end{tabular}




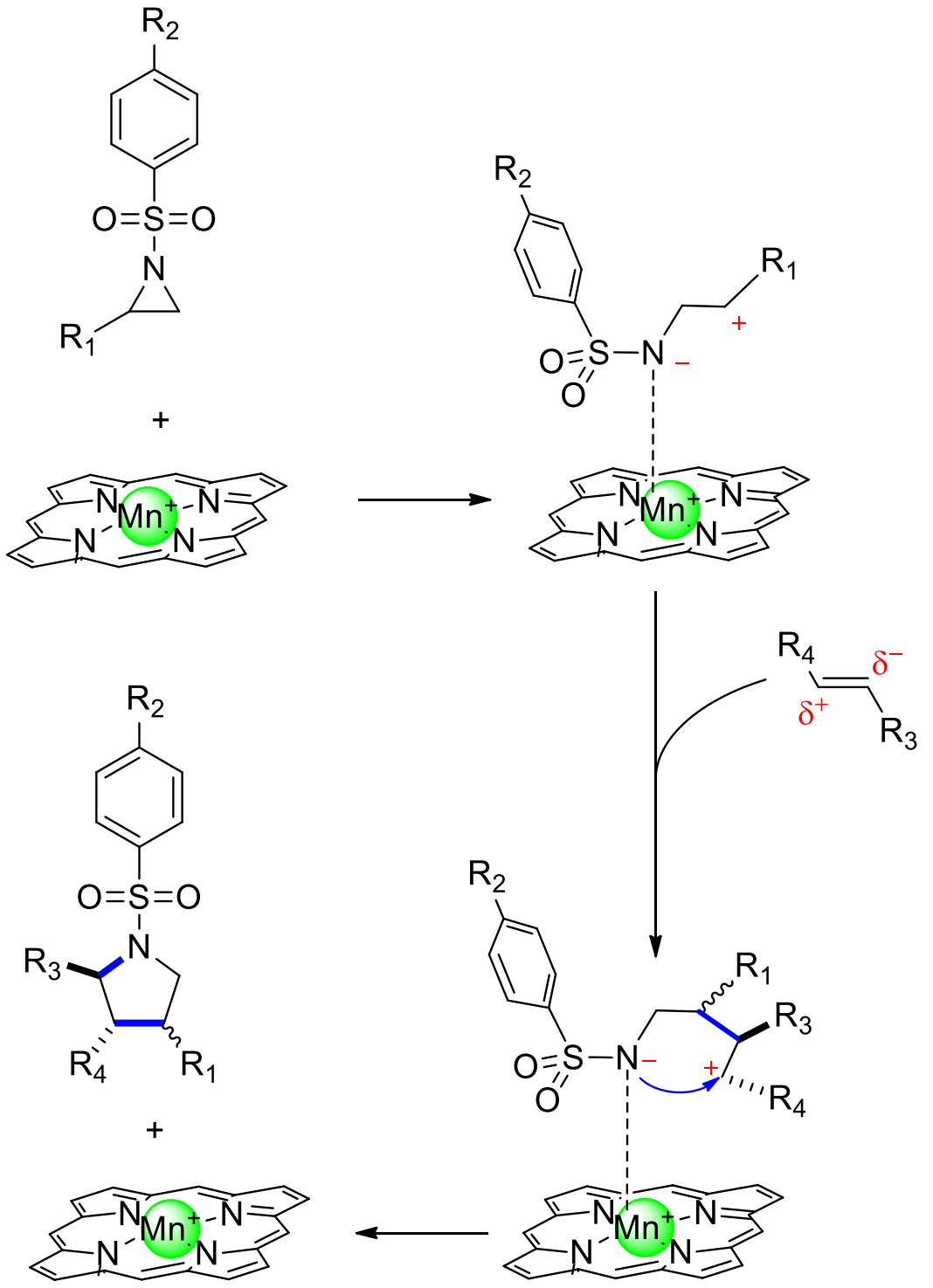

Scheme S5. Proposed mechanism for the [3+2] cycloaddition of aziridines and alkenes. 


\section{S4.1 General Procedure for the Preparation of Aziridine Substrates}

To demonstate the [3+2] cycloaddition of aziridines and alkenes occurs within the pores of UNLPF-14$\mathrm{Mn}^{\prime \prime \prime}$, the dimensions of the tetrakaidecahedral cage $(33.2 \AA \times 26.3 \AA \times 27.6 \AA)$ was estimated along with the dimmesions of the four hexagonal windows $(16.0 \AA \times 18.6 \AA)$ and eight pentagonal windows $(16.6 \AA$ $\times 12.9 \AA$ ) (Figure S9). Further, the largest possible circle fitting into both of the window types were estimated to have diameters of $13.2 \AA$ and $12.2 \AA$ (Figure S10a and $10 \mathrm{~b}$, respectively). To determine whether product molecules can freely diffuse out of the MOF, both the structures of the cis and trans isomers of all products were optimized and further fit into a rectangular pism of dimensions $d_{x}, d_{y}$, and $d_{z}$, where $d_{x}<d_{y}<d_{z}$ (Figure S10c) (see Nat. Commun. 2013, 4, 2344). For product diffusion to efficiently occur, the diagonal of $d_{x}$ and $d_{y}\left(d_{w}\right)$ should be smaller than the diameter of the window opening (13.2 $\AA$ ). For the cis and trans isomers of products $9 a-h, d_{w}$ was found to be smaller than $13.2 \AA$, indicating they can efficiently diffuse out of the MOF (Figure S11). However, $d_{w}$ for the cis and trans isomers of 9i was too large to pass through the cage windows.
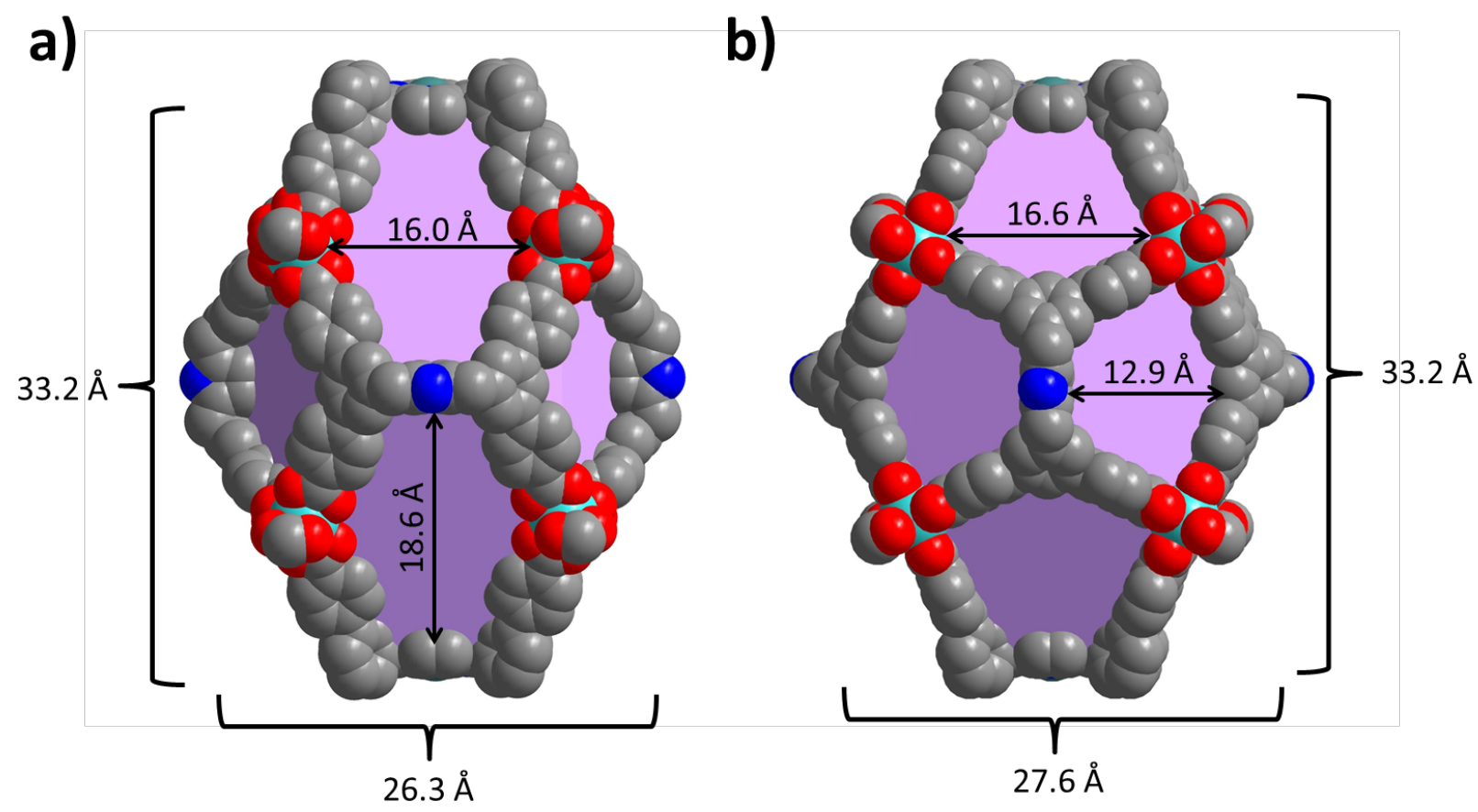

Figure 59. Tetrakaidechedral cage dimensions and hexagonal (a) and pentagonal (b) window dimensions for UNLPF-14-Mn'". 

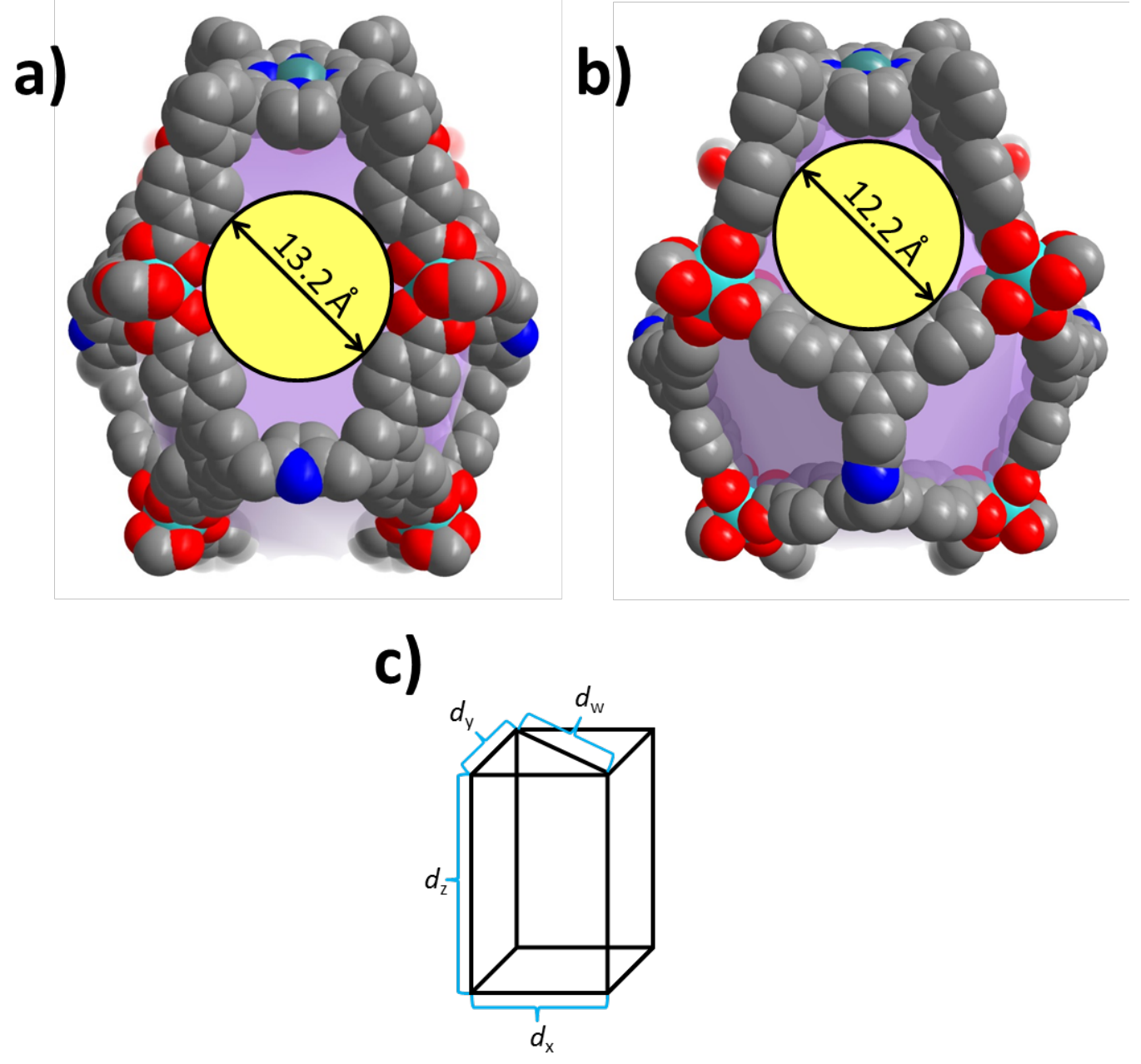

Figure S10. Estimatations of diameters for the largest circular opening in the hexagonal (a) and pentagonal (b) windows of the UNLPF-14-Mn"'. Substrates were fit into a rectangular prism with dimensions $d_{\mathrm{x}}, d_{\mathrm{y}}$, and $d_{\mathrm{z}}$, where $d_{\mathrm{x}}<d_{\mathrm{y}}<d_{\mathrm{z}}$ and $d_{\mathrm{w}}$ is the diagonal of $d_{\mathrm{x}}$ and $d_{\mathrm{y}}$. 


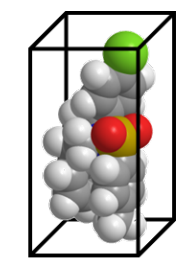

trans-9a

$d_{x}=5.90 \AA$

$d_{y}=7.59 \AA$

$d_{z}=12.59 \AA$

$d_{w}=9.62 \AA$

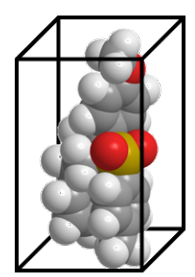

trans-9d

$d_{x}=5.61 \AA$

$d_{y}=7.76 \AA$

$d_{z}=12.85 \AA$

$d_{w}=9.57 \AA$

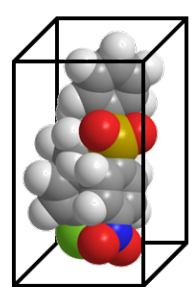

trans-9g

$d_{x}=5.90 \AA$

$d_{y}=8.08 \AA$

$d_{z}=12.30 \AA$

$d_{w}=10.01 \AA$

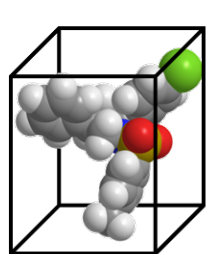

cis-9a

$d_{x}=6.61 \AA$

$d_{y}=9.65 \AA$

$d_{z}=9.85 \AA$

$d_{w}=11.70 \AA$

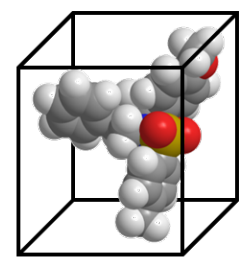

cis-9d

$d_{x}=7.45 \AA$

$d_{y}=7.79 \AA$

$d_{z}=11.86 \AA$

$d_{w}=10.77 \AA$

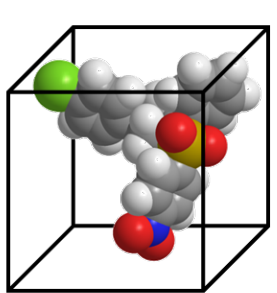

$$
\text { cis-9g }
$$

$d_{x}=8.19 \AA$

$d_{y}=9.27 \AA$

$d_{z}=9.32 \AA$

$d_{w}=12.35 \AA$

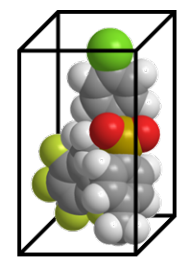

trans-9b

$d_{x}=5.71 \AA$

$d_{y}=7.87 \AA$

$d_{z}=12.89 \AA$

$d_{w}=9.72 \AA$

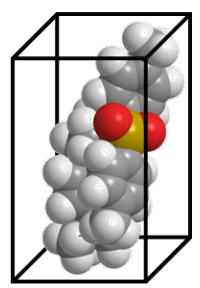

trans-9e

$d_{x}=8.08 \AA$

$d_{y}=10.15 \AA$

$d_{z}=13.33 \AA$

$d_{w}=12.97 \AA$

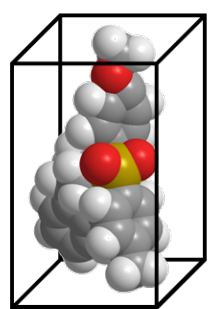

trans-9h

$d_{x}=6.66 \AA$

$d_{y}=10.81 \AA$

$d_{z}=12.31 \AA$

$d_{w}=12.70 \AA$

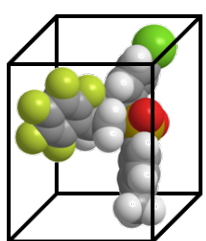

cis-9b

$d_{x}=6.89 \AA$

$d_{y}=7.53 \AA$

$d_{z}=9.85 \AA$

$d_{w}=11.84 \AA$

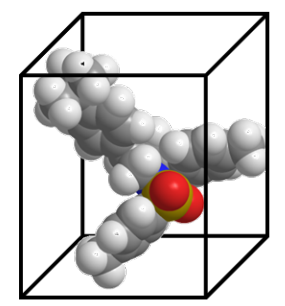

\section{cis-9e}

$d_{x}=9.00 \AA$

$d_{y}=9.50 \AA$

$d_{z}=9.92 \AA$

$d_{w}=13.08 \AA$

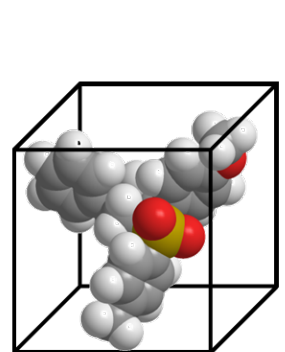

cis-9h

$d_{x}=8.12 \AA$

$d_{y}=9.18 \AA$

$d_{z}=9.27 \AA$

$d_{w}=12.25 \AA$

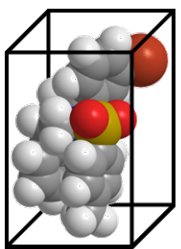

trans-9c

$d_{x}=6.25 \AA$

$d_{y}=7.76 \AA$

$d_{z}=12.72 \AA$

$d_{w}=9.96 \AA$

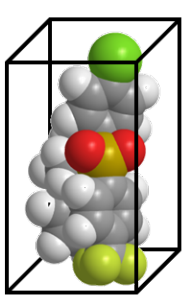

\section{trans-9f}

$d_{x}=7.71 \AA$

$d_{y}=9.27 \AA$

$d_{z}=12.07 \AA$

$d_{w}=12.08 \AA$

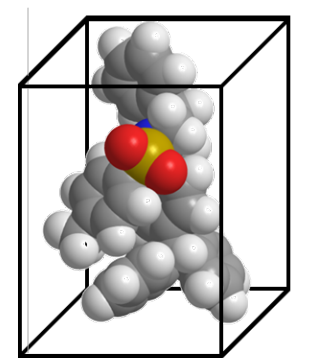

trans-9i

$d_{x}=11.75 \AA$

$d_{y}=12.41 \AA$

$d_{z}=14.60 \AA$

$d_{w}=17.09 \AA$

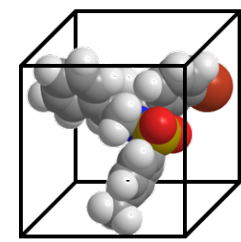

cis-9c

$d_{x}=6.52 \AA$

$d_{y}=7.81 \AA$

$d_{z}=10.96 \AA$

$d_{w}=10.28 \AA$

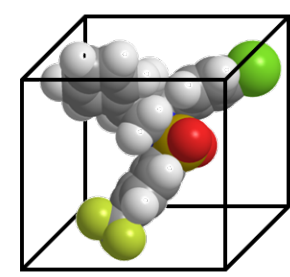

cis-9f

$d_{x}=8.00 \AA$

$d_{y}=8.61 \AA$

$d_{z}=9.83 \AA$

$d_{w}=11.76 \AA$

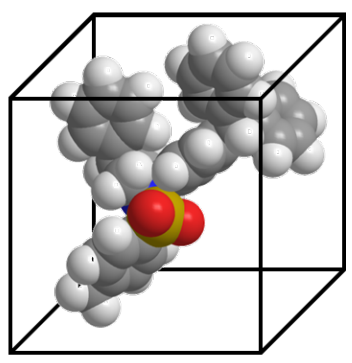

cis-9i

$d_{x}=10.09 \AA$

$d_{y}=14.81 \AA$

$d_{z}=23.35 \AA$

$d_{w}=17.91 \AA$

Figure S11. Estimations of the product dimensions $\left(d_{x}, d_{y}, d_{z}\right.$, and $\left.d_{w}\right)$ for products 9a-i. 


\section{S4.5 Test for Catalyst Heterogeneity}

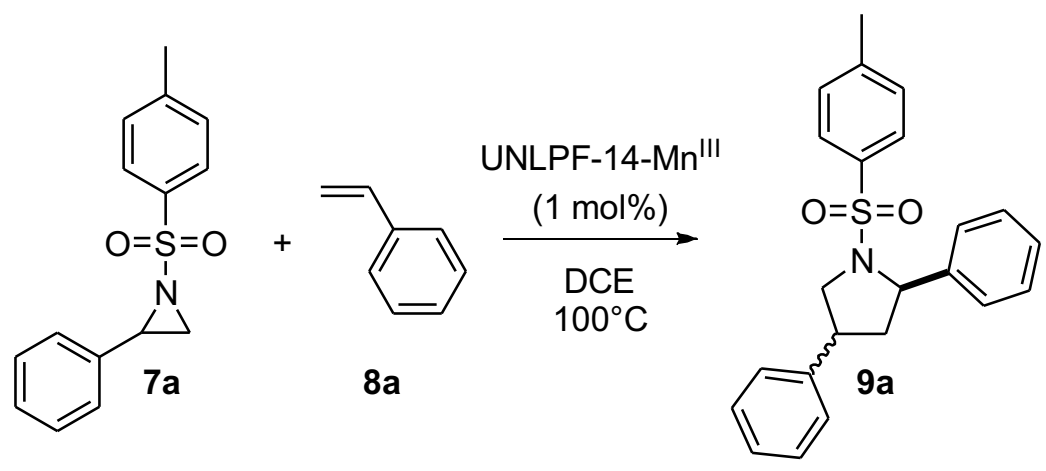

\begin{tabular}{|c|c|c|c|}
\hline & & $\begin{array}{l}\text { 1. Quench reaction } \\
\text { and check conversion immediately }\end{array}$ & $58 \%$ conversion \\
\hline Reaction & $\begin{array}{l}\text { Heated at } \\
100 \mathrm{C} \text { under } \\
\text { Ar for } 4 \text { hrs. }\end{array}$ & $\begin{array}{l}\text { 2. Filter the reaction mixture and allow } \\
\text { supernatant to be heated at } 100 \mathrm{C} \text { under } \\
\text { Ar for an additional } 4 \mathrm{hrs} \text {. }\end{array}$ & $60 \%$ conversion \\
\hline & & $\begin{array}{l}\text { 3. Without filtering, allow reaction } \\
\text { mixture to be heated at } 100 \mathrm{C} \text { under } \mathrm{Ar} \\
\text { for an additional } 4 \mathrm{hrs} \text {. Filter and } \\
\text { determine catalyst leaching in the } \\
\text { supernatent by ICP-MS. }\end{array}$ & $\begin{array}{l}96 \% \text { conversion } \\
0.05 \text { mol\% catalyst } \\
\text { leaching determined } \\
\text { ICP-MS }\end{array}$ \\
\hline
\end{tabular}

Scheme S6. Heterogeneity test for UNLPF-16-Fe"' catalyzing the [3+2] cycloaddition of 7a and 8a. 


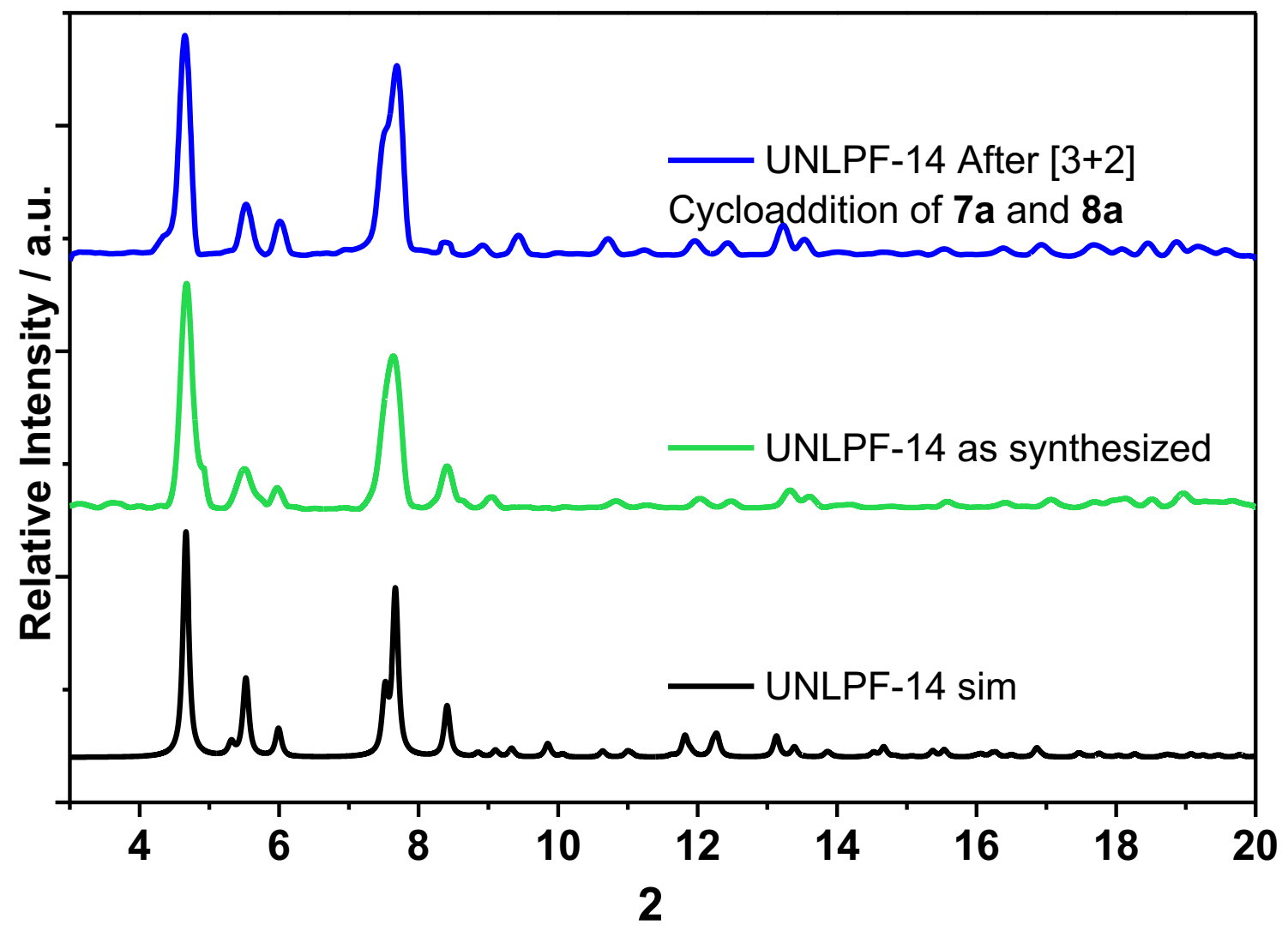

Figure S12. Powder X-ray diffraction patterns of UNLPF-14 simulated (black), as synthesized (green), and after the [3+2] cycloaddition of $7 a$ and 8 a (blue). 


\section{S4.6 Crystallographic Data for 9a and 9f}

Single crystal X-ray diffraction data was collected using synchrotron radiation, $\lambda=0.41328 \AA$, at the Advanced Photon Source, Chicago, IL. Indexing was performed using APEX2 (Difference Vectors method). Data integration and reduction were performed using SaintPlus 6.01. Absorption correction was performed by multi-scan method implemented in SADABS. ${ }^{3}$ Space groups were determined using XPREP implemented in APEX2. The structures were solved using the program XT and refined using SHELXL-2013 (full-matrix least-squares on $\mathrm{F}^{2}$ ) contained in APEX2 and WinGX v1.70.00. $.^{4-7} \mathrm{C}, \mathrm{N}, \mathrm{O}$ atoms were refined with anisotropic displacement parameters and $\mathrm{H}$ atoms were placed in geometrically calculated positions and included in the refinement process using riding model with isotropic thermal parameters: $U_{\text {iso }}(\mathrm{H})=$ $1.2 U_{\text {eq }}(-\mathrm{CH})$. Crystal data and refinement conditions are shown in Tables S6-S7. CCDC 1447710 and 1451926 contains the supplementary crystallographic data for compounds $9 a$ and $9 f$. This data can be obtained free of charge from The Cambridge Crystallographic Data Centre via www.ccdc.cam.ac.uk/data_request/cif.

$9 a$

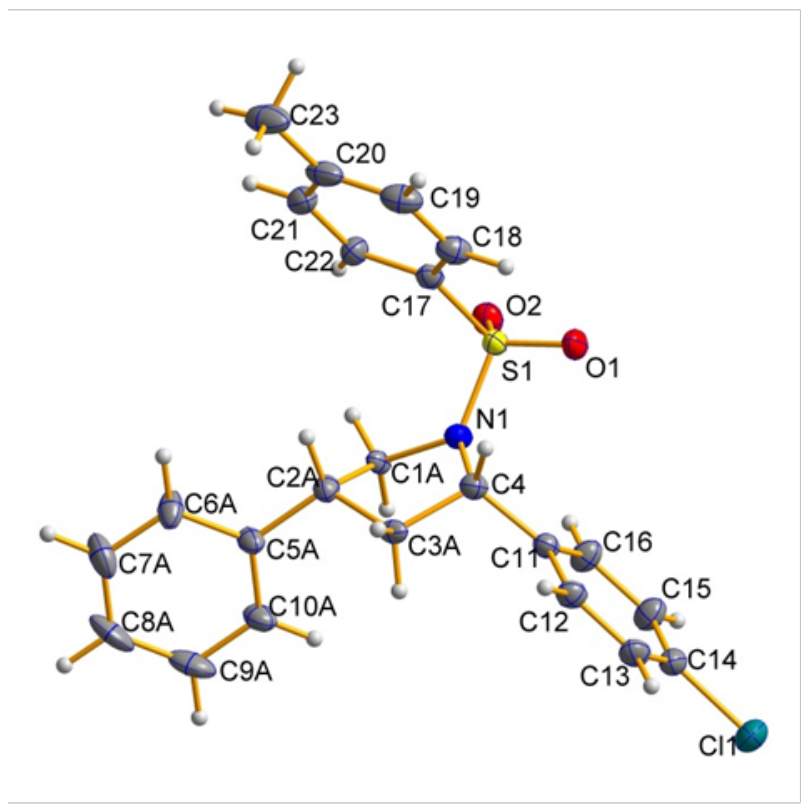

9f

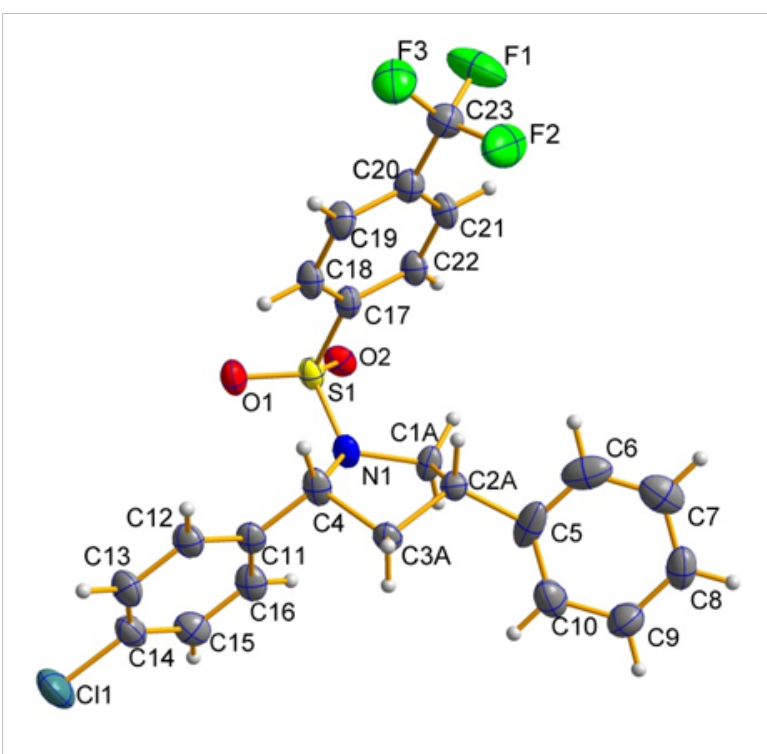

Figure S13. Thermal ellipsoid diagrams for compounds 9 a and $9 \mathrm{f}$ shown at the $50 \%$ proability level. 
Table S6.Crystal data and structure refinement for compound 9a

\begin{tabular}{|c|c|}
\hline Identification & $9 a$ \\
\hline empirical formula & $\mathrm{C}_{23} \mathrm{H}_{22} \mathrm{ClNO}_{2} \mathrm{~S}$ \\
\hline formula weight $\left(\mathrm{g} \cdot \mathrm{mol}^{-1}\right)$ & 411.93 \\
\hline temperature $(\mathrm{K})$ & $100(2) \mathrm{K}$ \\
\hline wavelength $(\AA)$ & 0.41328 \\
\hline crystal system, space group & monoclinic, $P 2_{1} / c$ \\
\hline$a(\AA)$ & $14.1702(5)$ \\
\hline$b(\AA)$ & $15.5119(6)$ \\
\hline$c(\AA)$ & $9.5178(4)$ \\
\hline$\alpha(\mathrm{deg})$ & 90.00 \\
\hline$\beta$ (deg) & $105.3279(11)$ \\
\hline$\gamma(\operatorname{deg})$ & 90.00 \\
\hline volume $\left(\AA^{3}\right)$ & $2017.66(14)$ \\
\hline$Z$, density (calcd) $\left(\mathrm{Mg} \cdot \mathrm{m}^{-3}\right)$ & $4,1.356$ \\
\hline abs coefficient $\left(\mathrm{mm}^{-1}\right)$ & 0.082 \\
\hline$F(000)$ & 864.0 \\
\hline crystal size $\left(\mathrm{mm}^{3}\right)$ & $0.1 \times 0.3 \times 0.2 \mathrm{~mm}$ \\
\hline$\theta$ range $(\mathrm{deg})$ & $0 . .87$ to $24.51 \mathrm{deg}$ \\
\hline reflections collected/unique & $14178 / 11327$ \\
\hline completeness to $\theta(\%)$ & $82.9 \%$ \\
\hline refinement method & Full-matrix least-squares on $F^{2}$ \\
\hline data / restraints / parameters & 14178 / 96 / 336 \\
\hline goodness-of-fit on $F^{2}$ & 1.556 \\
\hline final Rindices [I>2sigma(I)] & $R_{1}=0.0648, w R_{2}=0.2296$ \\
\hline largest diff. peak and hole (e. $\mathrm{A}^{-3}$ ) & 1.22 and -1.08 \\
\hline
\end{tabular}


Table S7.Crystal data and structure refinement for compound $9 f$

\begin{tabular}{|c|c|}
\hline Identification & $9 f$ \\
\hline empirical formula & $\mathrm{C}_{23} \mathrm{H}_{19} \mathrm{ClF}_{3} \mathrm{NO}_{2} \mathrm{~S}$ \\
\hline formula weight $\left(\mathrm{g} \cdot \mathrm{mol}^{-1}\right)$ & 465.90 \\
\hline temperature $(\mathrm{K})$ & $100(2) \mathrm{K}$ \\
\hline wavelength $(\AA)$ & 0.41328 \\
\hline crystal system, space group & monoclinic, $P 2_{1} / c$ \\
\hline$a(\AA)$ & $15.6602(17)$ \\
\hline$b(\AA)$ & $18.7951(19)$ \\
\hline$c(\AA)$ & $7.4123(8)$ \\
\hline$\alpha$ (deg) & 90.00 \\
\hline$\beta$ (deg) & $99.029(3)$ \\
\hline$\gamma(\operatorname{deg})$ & 90.00 \\
\hline volume $\left(\AA^{3}\right)$ & $2154.7(4)$ \\
\hline$Z$, density (calcd) $\left(\mathrm{Mg} \cdot \mathrm{m}^{-3}\right)$ & $4,1.436$ \\
\hline abs coefficient $\left(\mathrm{mm}^{-1}\right)$ & 0.084 \\
\hline$F(000)$ & 960.0 \\
\hline crystal size $\left(\mathrm{mm}^{3}\right)$ & $0.1 \times 0.3 \times 0.2 \mathrm{~mm}$ \\
\hline$\theta$ range $(\mathrm{deg})$ & 1.32 to $31.73 \mathrm{deg}$ \\
\hline reflections collected/unique & 7185 / 4932 \\
\hline completeness to $\theta(\%)$ & $98.4 \%$ \\
\hline refinement method & Full-matrix least-squares on $F^{2}$ \\
\hline data / restraints / parameters & 7185 / 0 / 309 \\
\hline goodness-of-fit on $F^{2}$ & 1.14 \\
\hline final Rindices [I>2sigma(I)] & $R_{1}=0.0613, w R_{2}=0.1814$ \\
\hline largest diff. peak and hole (e. $\left.\mathrm{A}^{-3}\right)$ & 0.654 and -0.585 \\
\hline
\end{tabular}


Section S5 [4+2] Hetero-Diels- Alder Reaction

\section{S5.1 General Procedure for the Hetero-Diels-Alder Reaction}

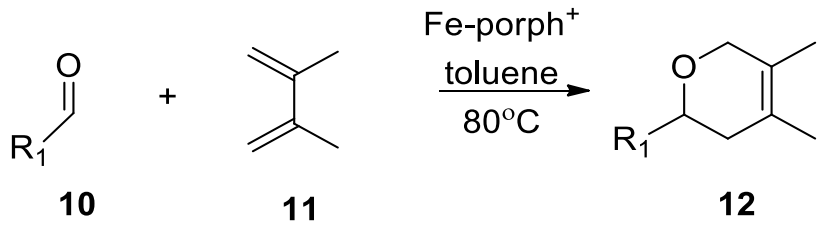

Under a dry argon atomosphere in a glove bag, a solution of the respective aldehyde $(0.5 \mathrm{mmol})$, and 2,3-Dimethyl-1,3-butadiene $(2.0 \mathrm{mmol})$ in anhydrous toluene $(2.0 \mathrm{~mL})$ was added to a screw cap vial equipped with a stir bar and UNLPF-16 $(5 \mathrm{mg})$. The vial was sealed with a silicone screw cap septum and heated at $80^{\circ} \mathrm{C}$. Reaction progress was monitored by TLC (hexanes/diethyl ether v/v 30:1). Upon disappearance of the aldehyde starting material, the reaction mixture was cooled to room temperature, diluted with dichloromethane $(10 \mathrm{~mL})$ and absorbed onto silica. The crude reaction mixture was purified by flash chromatography using a silica gel column with an elution of hexanes/diethyl ether (v/v 30:1). The obtained organic fraction was concetrated in vacuo to give the desired pure product.

\section{4,5-dimethyl-2-phenyl-3,6-dihydro-2H-pyran (12a).}<smiles>CC1=C(C)CC(c2ccccc2)OC1</smiles>

${ }^{1} \mathbf{H}$ NMR $\left(300 \mathrm{MHz}, \mathrm{CDCl}_{3}\right) \delta 7.41-7.26(\mathrm{~m}, 5 \mathrm{H}, \mathrm{ArH}), 4.54(\mathrm{dd}, J=10.5,3.5 \mathrm{~Hz}, 1 \mathrm{H}, \mathrm{H}-2), 4.21$ (d, J = 15.8 $\mathrm{Hz}, 1 \mathrm{H}, \mathrm{H}-6 \mathrm{a}), 4.11$ (d, J = $15.0 \mathrm{~Hz}, 1 \mathrm{H}, \mathrm{H}-6 \mathrm{~b}), 2.37-2.23(\mathrm{~m}, 1 \mathrm{H}, \mathrm{H}-3 \mathrm{a}), 2.09$ (d, J = $17.0 \mathrm{~Hz}, 1 \mathrm{H}, \mathrm{H}-3 \mathrm{~b})$, $1.69\left(\mathrm{~s}, 3 \mathrm{H}, 5-\mathrm{CH}_{3}\right), 1.60\left(\mathrm{~s}, 3 \mathrm{H}, 4-\mathrm{CH}_{3}\right)$.

${ }^{13} \mathrm{C}$ NMR $\left(75 \mathrm{MHz}, \mathrm{CDCl}_{3}\right) \delta 142.80,128.50,127.53,125.98,124.70,124.01,76.49,70.48,38.71,18.50$, 14.01.

HR-EI MS found for $\left[\mathrm{M}^{++}\right] \mathrm{m} / \mathrm{z}$ 188.1195; calculated for $\left[\mathrm{M}^{++}\right] \mathrm{m} / \mathrm{z}$ 188.1201.

\section{2-(4-bromophenyl)-4,5-dimethyl-3,6-dihydro-2H-pyran (12b).}<smiles>CC1=C(C)CC(c2ccc(Br)cc2)OC1</smiles> 
${ }^{1}$ H NMR (300 MHz, CDCl $)$ ) 7.47 (d, J = 8.4 Hz, 2H, Ar H-3,5), 7.25 (d, J = 8.3 Hz, 3H, Ar H-2,6), 4.50 (dd, J $=10.4,3.7 \mathrm{~Hz}, 1 \mathrm{H}, \mathrm{H}-2), 4.19$ (d, J = $15.4 \mathrm{~Hz}, 1 \mathrm{H}, \mathrm{H}-6 \mathrm{a}), 4.09$ (d, J = 15.6 Hz, 1H, H-6b), $2.30-2.16(\mathrm{~m}, 1 \mathrm{H}$, $\mathrm{H}-3 \mathrm{a}), 2.06(\mathrm{~d}, \mathrm{~J}=16.6 \mathrm{~Hz}, 1 \mathrm{H}, 1 \mathrm{H}, \mathrm{H}-3 \mathrm{~b}), 1.68\left(\mathrm{~s}, 3 \mathrm{H}, 5-\mathrm{CH}_{3}\right), 1.59\left(\mathrm{~s}, 3 \mathrm{H}, 4-\mathrm{CH}_{3}\right)$.

${ }^{13} \mathrm{C}$ NMR $\left(75 \mathrm{MHz}, \mathrm{CDCl}_{3}\right) \delta 141.76,131.42,127.54,124.60,123.60,121.11,75.57,70.23,38.46,18.34$, 13.85.

ESI MS found for $\left[\mathrm{M}^{++}\right] \mathrm{m} / \mathrm{z} 266.0304$; calculated for $\left[\mathrm{M}^{++}\right] \mathrm{m} / \mathrm{z} 266.0306$.

\section{4,5-dimethyl-2-(p-tolyl)-3,6-dihydro-2H-pyran (12c).}<smiles>CC1=C(C)CC(c2ccc(C)cc2)OC1</smiles>

${ }^{1}$ H NMR $\left(300 \mathrm{MHz}, \mathrm{CDCl}_{3}\right) \delta 7.26$ (d, J=8.0 Hz, 2H, Ar H-2,6), 7.15 (d, J = 7.9 Hz, 2H, Ar H-3,5), 4.51 (dd, J $=10.5,3.4 \mathrm{~Hz}, 1 \mathrm{H}, \mathrm{H}-2), 4.20$ (d, J=15.8 Hz, 1H, H-6a), 4.09 (d, J = 15.6 Hz, 1H, H-6b), 2.34 (s, 3H, Ar-CH , , $2.31-2.22(\mathrm{~m}, 1 \mathrm{H}, \mathrm{H}-3 \mathrm{a}), 2.06(\mathrm{~d}, \mathrm{~J}=16.7 \mathrm{~Hz}, 1 \mathrm{H}, \mathrm{H}-3 \mathrm{~b}), 1.68\left(\mathrm{~s}, 3 \mathrm{H}, 5-\mathrm{CH}_{3}\right), 1.59\left(\mathrm{~s}, 3 \mathrm{H}, 4-\mathrm{CH}_{3}\right)$.

${ }^{13} \mathrm{C}$ NMR $\left(75 \mathrm{MHz}, \mathrm{CDCl}_{3}\right) \delta 139.82,129.16,125.95,124.68,124.05,110.62,76.35,70.47,38.67,21.28$, 18.51, 14.00 .

HR-EI MS found for $\left[\mathrm{M}^{++}\right] \mathrm{m} / \mathrm{z}$ 202.1365; calculated for $\left[\mathrm{M}^{++}\right] \mathrm{m} / \mathrm{z} 202.1358$.

4-(4,5-dimethyl-3,6-dihydro-2H-pyran-2-yl)benzonitrile (12d).<smiles>CC1=C(C)CC(c2ccc(C#N)cc2)OC1</smiles>

${ }^{1} \mathrm{H}$ NMR $\left(400 \mathrm{MHz}, \mathrm{CDCl}_{3}\right) \delta 7.63(\mathrm{~d}, J=8.2 \mathrm{~Hz}, 2 \mathrm{H}, \mathrm{Ar} \mathrm{H}-3,5), 7.47$ (d, J = 8.1 Hz, 2H, Ar H-3,5), 4.58 (dd, J $=10.3,3.5 \mathrm{~Hz}, 1 \mathrm{H}, \mathrm{H}-2), 4.20$ (d, J = 15.6 Hz, 1H, H-6a), 4.11 (d, J = 15.4 Hz, 1H, H-6b), $2.27-2.14(\mathrm{~m}, 1 \mathrm{H}$, $\mathrm{H}-3 \mathrm{a}), 2.10$ (d, J = $16.3 \mathrm{~Hz}, 1 \mathrm{H}, \mathrm{H}-3 \mathrm{~b}), 1.69\left(\mathrm{~s}, 3 \mathrm{H}, 5-\mathrm{CH}_{3}\right), 1.59\left(\mathrm{~s}, 3 \mathrm{H}, 4-\mathrm{CH}_{3}\right)$.

${ }^{13} \mathrm{C}$ NMR $\left(101 \mathrm{MHz}, \mathrm{CDCl}_{3}\right) \delta 148.23,132.31,126.46,124.80,123.45,119.06,111.14,75.47,70.24,38.47$, 18.43, 13.95.

HR-EI MS found for $\left[\mathrm{M}^{+*}\right] \mathrm{m} / \mathrm{z} 213.1151$; calculated for $\left[\mathrm{M}^{+}\right] \mathrm{m} / \mathrm{z} 213.1154$. 


\section{4,5-dimethyl-2-(4-nitrophenyl)-3,6-dihydro-2H-pyran (12e).}<smiles>CC1=C(C)CC(c2ccc([N+](=O)[O-])cc2)OC1</smiles>

${ }^{1}$ H NMR (300 MHz, CDCl $) \delta 8.20(\mathrm{~d}, J=8.8 \mathrm{~Hz}, 2 \mathrm{H}, \mathrm{Ar} \mathrm{H}-3,5), 7.53$ (d, J=8.7 Hz, 2H, Ar H-2,6), 4.64 (dd, J $=9.9,4.3 \mathrm{~Hz}, 1 \mathrm{H}, \mathrm{H}-2), 4.21(\mathrm{~d}, J=15.9 \mathrm{~Hz}, 1 \mathrm{H}, \mathrm{H}-6 \mathrm{a}), 4.12(\mathrm{~d}, J=15.5 \mathrm{~Hz}, 1 \mathrm{H}, \mathrm{H}-6 \mathrm{~b}), 2.26-2.06(\mathrm{~m}, 2 \mathrm{H}$, $\mathrm{H}-3), 1.69\left(\mathrm{~s}, 3 \mathrm{H}, 5-\mathrm{CH}_{3}\right), 1.60\left(\mathrm{~s}, 3 \mathrm{H}, 4-\mathrm{CH}_{3}\right)$.

${ }^{13} \mathrm{C}$ NMR $\left(75 \mathrm{MHz}, \mathrm{CDCl}_{3}\right) \delta 150.17,147.15,126.38,124.72,123.61,123.29,75.18,70.13,38.42,18.32$, 13.85 .

ESI MS found for $\left[\mathrm{M}^{+}\right] \mathrm{m} / \mathrm{z} 233.1052$; calculated for $\left[\mathrm{M}^{++}\right] \mathrm{m} / \mathrm{z} 233.1062$. 
Table S8. [4+2] Hetero-Diels-Alder Cycloaddition of Aldehydes with Dienes

\begin{tabular}{|c|c|c|c|c|c|}
\hline \multirow[b]{2}{*}{ entry $^{a}$} & \multicolumn{5}{|c|}{ 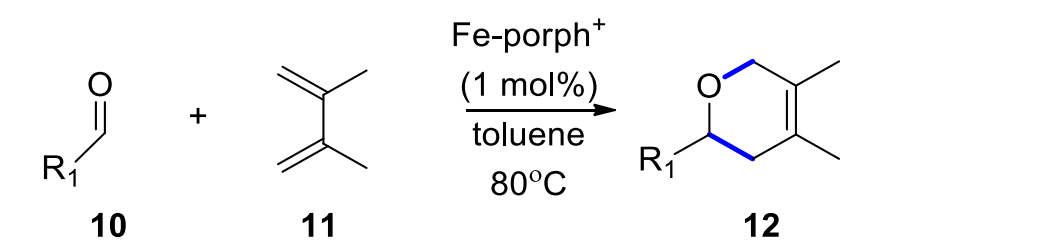 } \\
\hline & catalyst & $\mathrm{R}_{1}$ & product & $t(h)$ & yield $(\%)^{b}$ \\
\hline 1 & UNLPF-15-Fe" & $\mathrm{Ph}$ & $12 a$ & 12 & n.r. \\
\hline 2 & UNLPF-16-Fe ${ }^{I I I}$ & $\mathrm{Ph}$ & $12 a$ & 8 & $>99(96)$ \\
\hline 3 & $\mathrm{PCN}-223-\mathrm{Fe} \mathrm{e}^{\mathrm{III} \mathrm{Cl}}$ & $\mathrm{Ph}$ & $12 a$ & 24 & n.r. \\
\hline $4^{c}$ & $\left.\mathrm{PCN}-223-\mathrm{Fe}^{\mathrm{III}}\left[\mathrm{BF}_{4}\right]\right]$ & $\mathrm{Ph}$ & $12 a$ & 12 & $>99$ \\
\hline $5^{d}$ & $\mathrm{FeTPP}\left[\mathrm{BF}_{4}\right]$ & $\mathrm{Ph}$ & $12 a$ & 12 & 92 \\
\hline 6 & $2 \mathrm{c}-\mathrm{Fe}$ & $\mathrm{Ph}$ & $12 a$ & 12 & 90 \\
\hline 7 & UNLPF-16-Fe ${ }^{I I I}$ & $4 \mathrm{Br}-\mathrm{Ph}$ & $12 b$ & 10 & $97(94)$ \\
\hline 8 & UNLPF-16-Fe ${ }^{I I I}$ & $4 \mathrm{Me}-\mathrm{Ph}$ & $12 \mathrm{c}$ & 8 & $>99(97)$ \\
\hline 9 & UNLPF-16-Fe ${ }^{I I I}$ & $4 \mathrm{CN}-\mathrm{Ph}$ & $12 d$ & 18 & $97(92)$ \\
\hline $10^{c}$ & $\mathrm{PCN}-223-\mathrm{Fe}^{\mathrm{III}}\left[\mathrm{BF}_{4}\right]$ & $4 \mathrm{CN}-\mathrm{Ph}$ & $12 d$ & 12 & 30 \\
\hline 11 & UNLPF-16-Fe ${ }^{I I I}$ & $4 \mathrm{NO}_{2}-\mathrm{Ph}$ & $12 e$ & 48 & $87(83)$ \\
\hline 12 & $\mathrm{PCN}-223-\mathrm{Fe}^{\mathrm{III}}\left[\mathrm{BF}_{4}\right]$ & $4 \mathrm{NO}_{2}-\mathrm{Ph}$ & $12 e$ & 48 & 22 \\
\hline 13 & $\mathrm{FeTPP}\left[\mathrm{BF}_{4}\right]$ & $4 \mathrm{NO}_{2}-\mathrm{Ph}$ & $12 \mathrm{e}$ & 48 & 15 \\
\hline $14^{\mathrm{e}}$ & UNLPF-16-Fe ${ }^{\prime \prime \prime}$ & $\mathrm{Ph}$ & $12 a$ & 8 & 97 \\
\hline $\begin{array}{l}{ }^{\mathrm{a}} \text { Reaction } \\
\text { toluene at } \\
\text { loading }=\end{array}$ & $\begin{array}{l}\text { ns: Fe-porph }{ }^{+} \text {catalyst }(1 r \\
\text { Determined by }{ }^{1} \mathrm{H} \text { NMR, is } \\
\text { benzene was used as the }\end{array}$ & ${ }^{\circ}$ After $3^{\text {ro }}$ & ol), and c & $.00 \mathrm{mr}$ & $\begin{array}{l}2.0 \mathrm{~mL} \\
\text { f. } 24, \text { catalyst }\end{array}$ \\
\hline
\end{tabular}



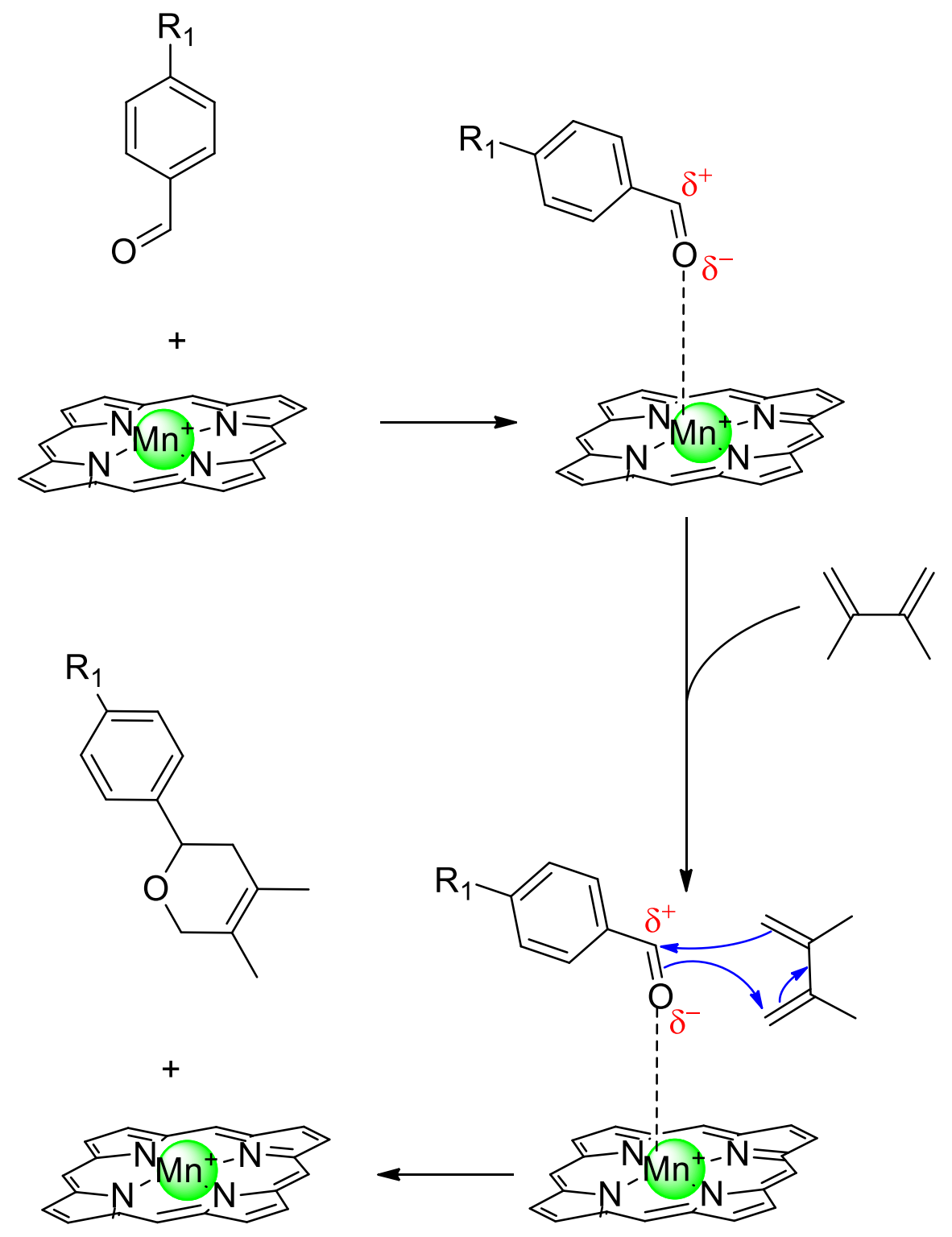

Scheme S7. Proposed mechanism for the [4+2] hetero-Diels-Alder reaction. 


\section{S5.2 Test for Catalyst Heterogeneity}
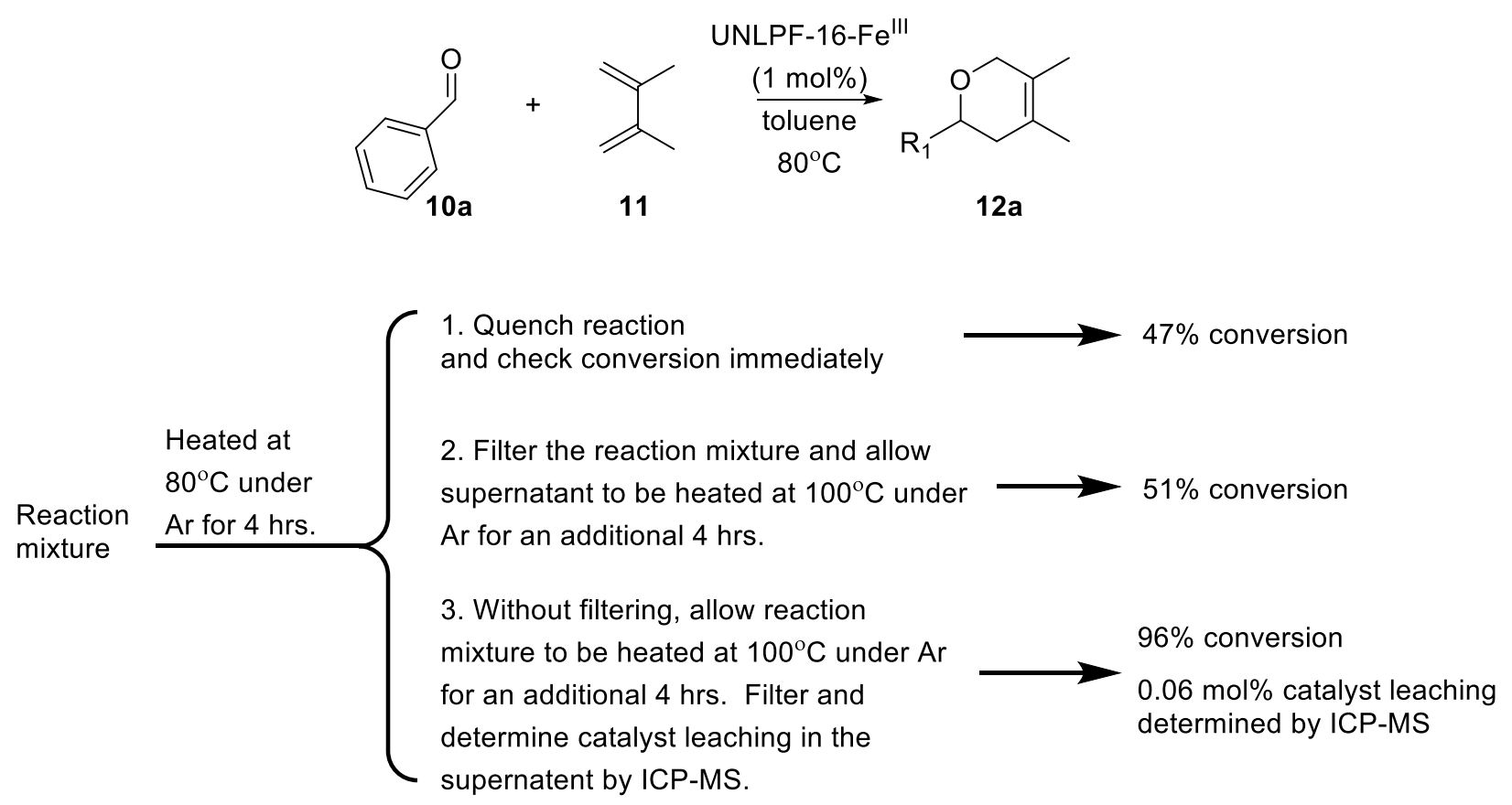

Scheme S8. Heterogeneity test for UNLPF-16-Fe"II catalyzing the [4+2] hetero-Diels-Alder cycloaddition of $10 \mathrm{a}$ and 11. 


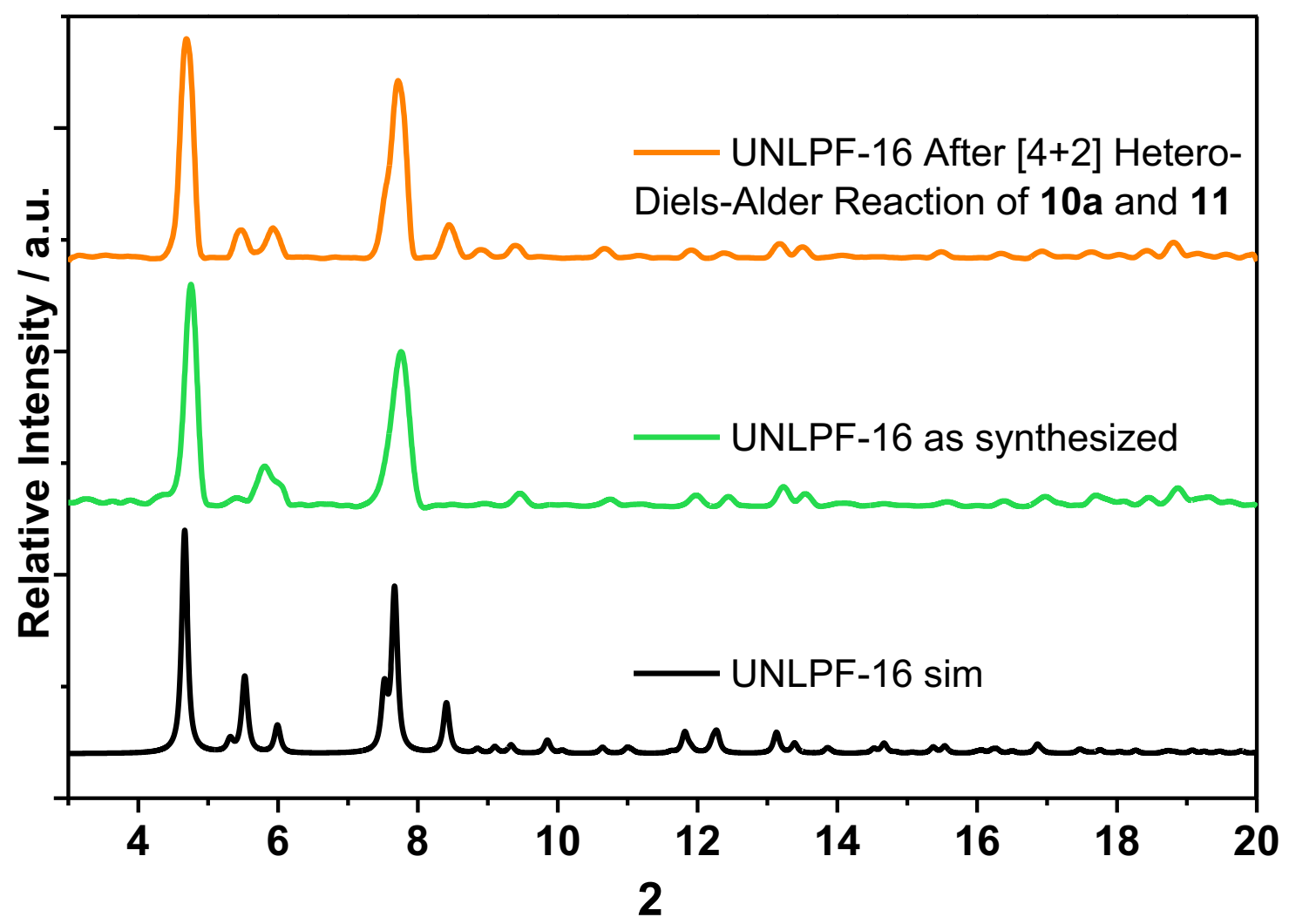

Figure S14. Powder X-ray diffraction patterns of UNLPF-16 simulated (black), as synthesized (green), and after the [4+2] hetero-Diels-Alder reaction of 10a and 11 (orange). 
Section S6 ${ }^{1} H$ NMR and ${ }^{13} C N M R$ Spectra

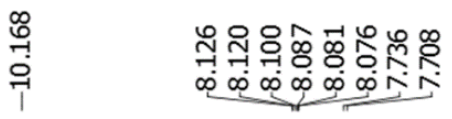

$\stackrel{\overrightarrow{0}}{\vec{i}}$
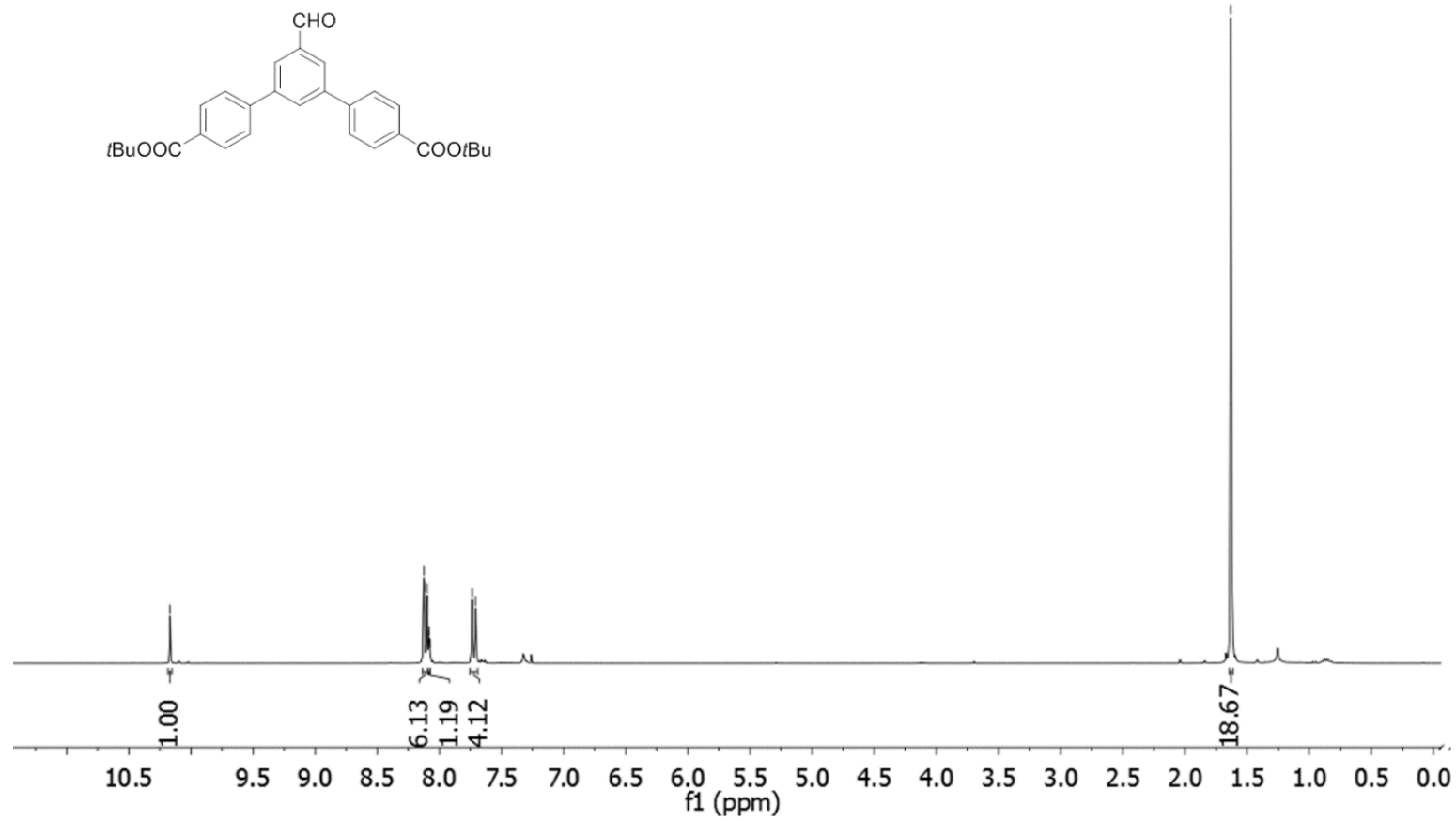

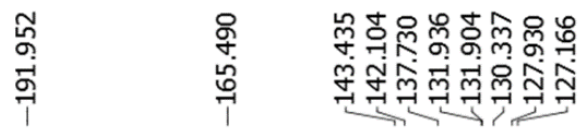
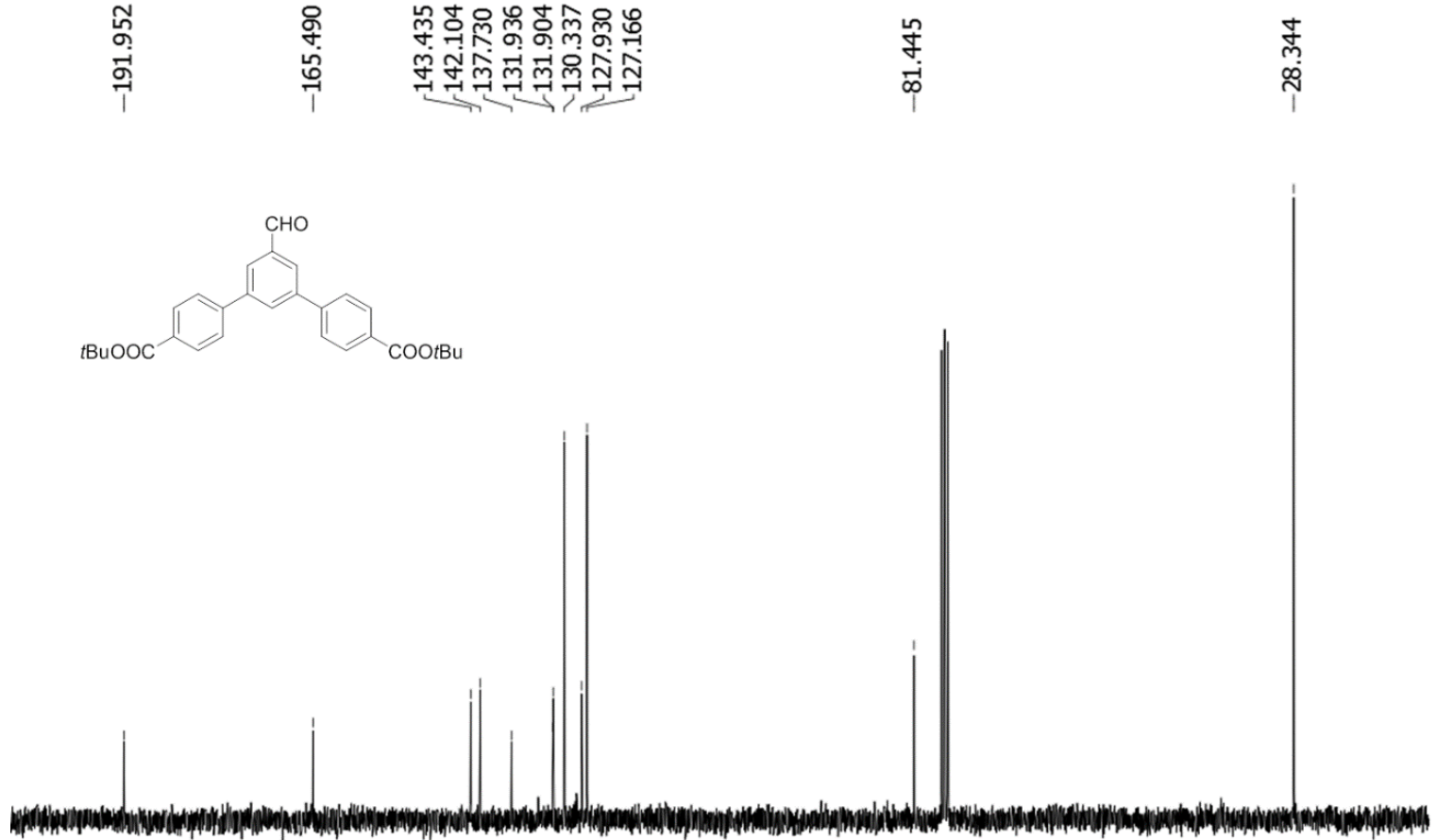


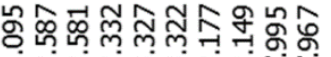

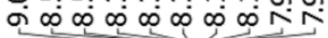

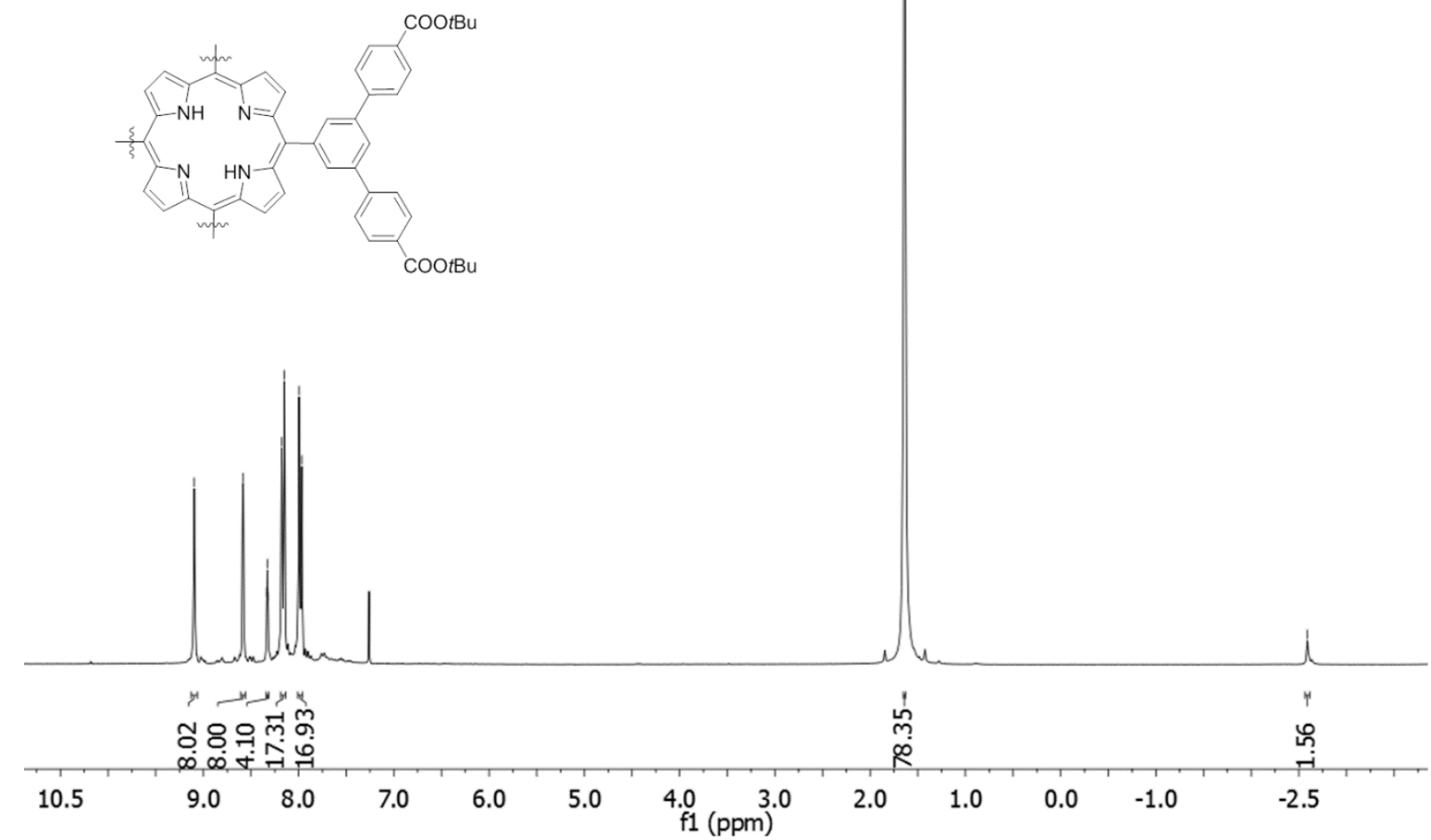

$\underset{+\infty}{+\infty}$

గి

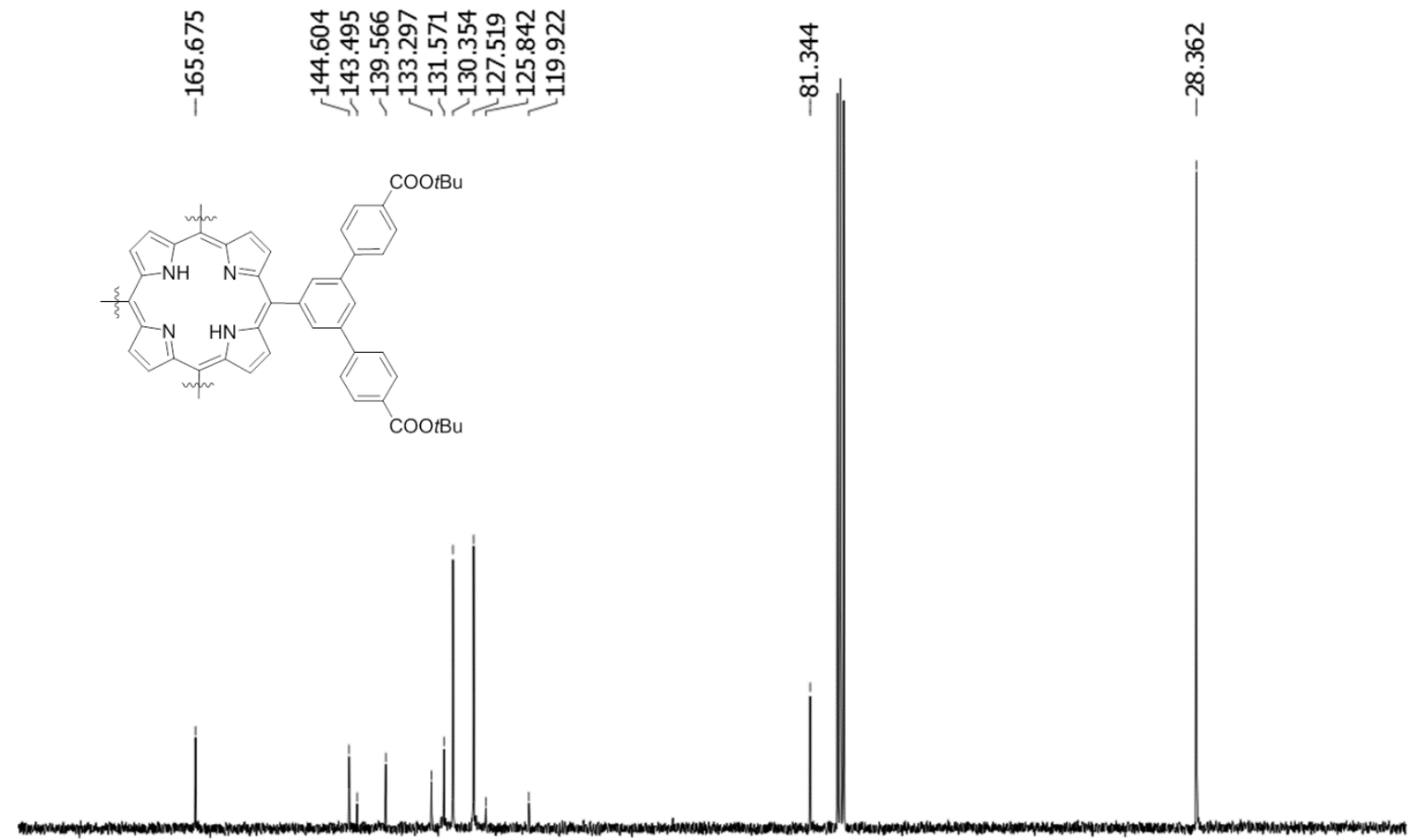

$\begin{array}{llllllllllllllllll}180 & 170 & 160 & 150 & 140 & 130 & 120 & 110 & \begin{array}{c}100 \\ \mathrm{f} 1(\mathrm{ppm})\end{array} & 80 & 70 & 60 & 50 & 40 & 30 & 20 & 10 & 0\end{array}$

S- 61 - 


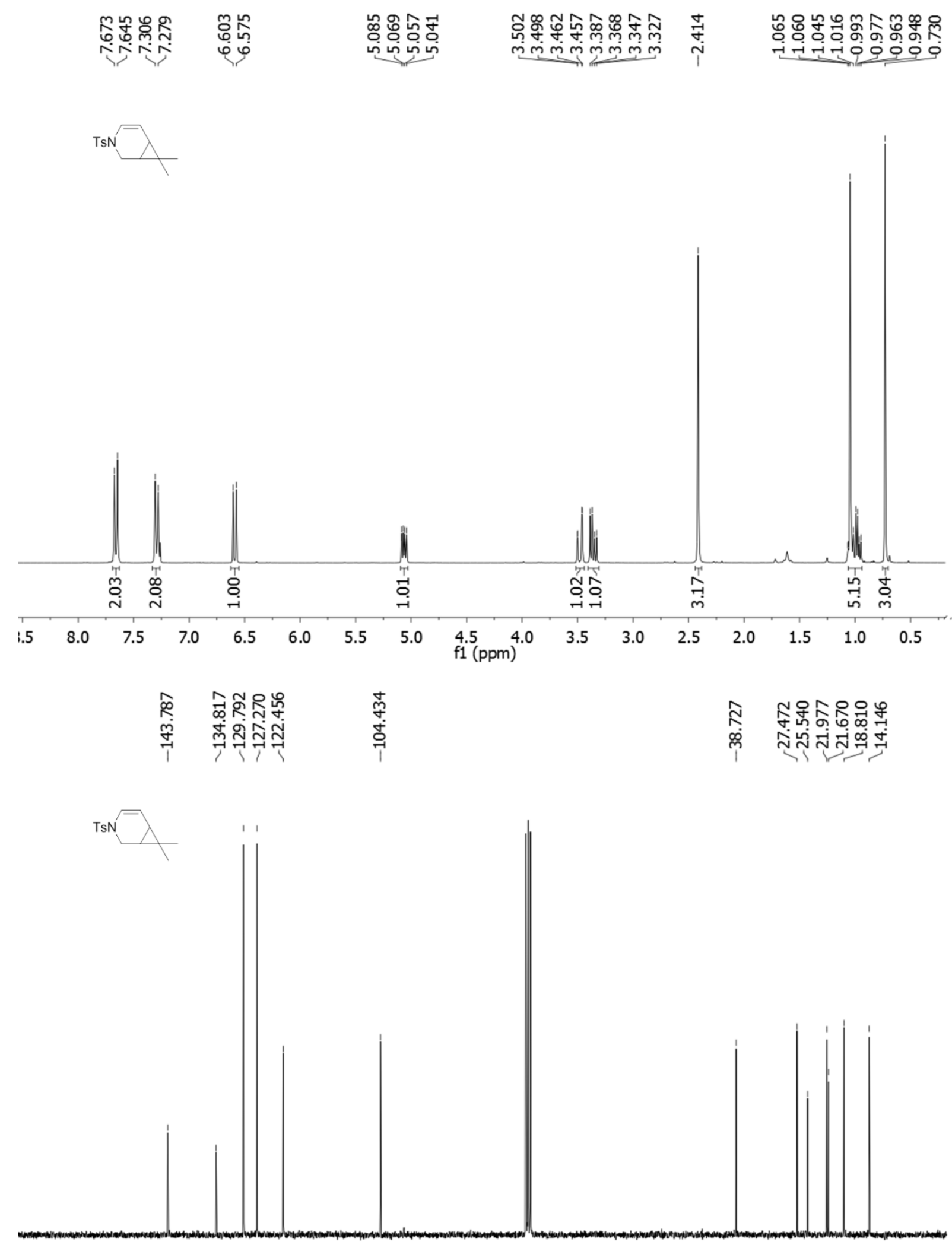

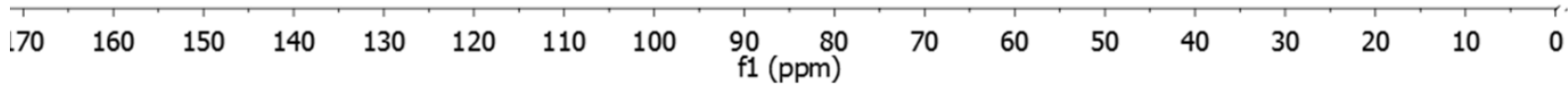




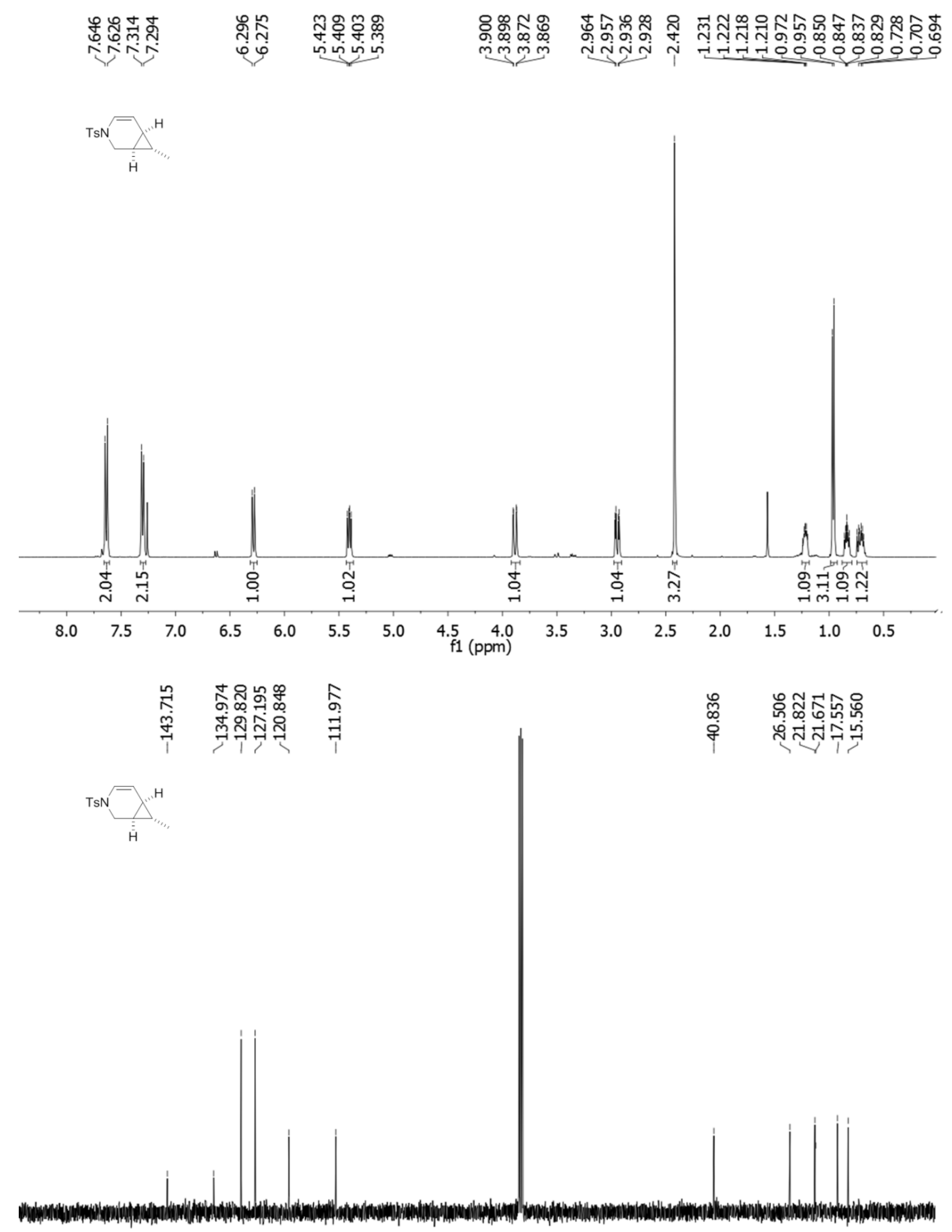

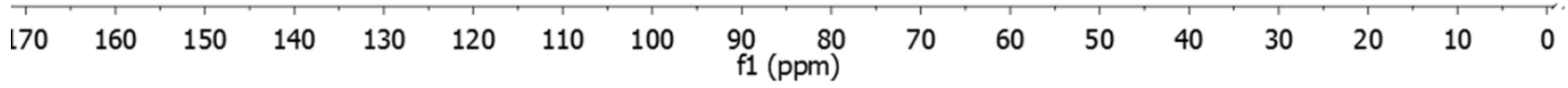



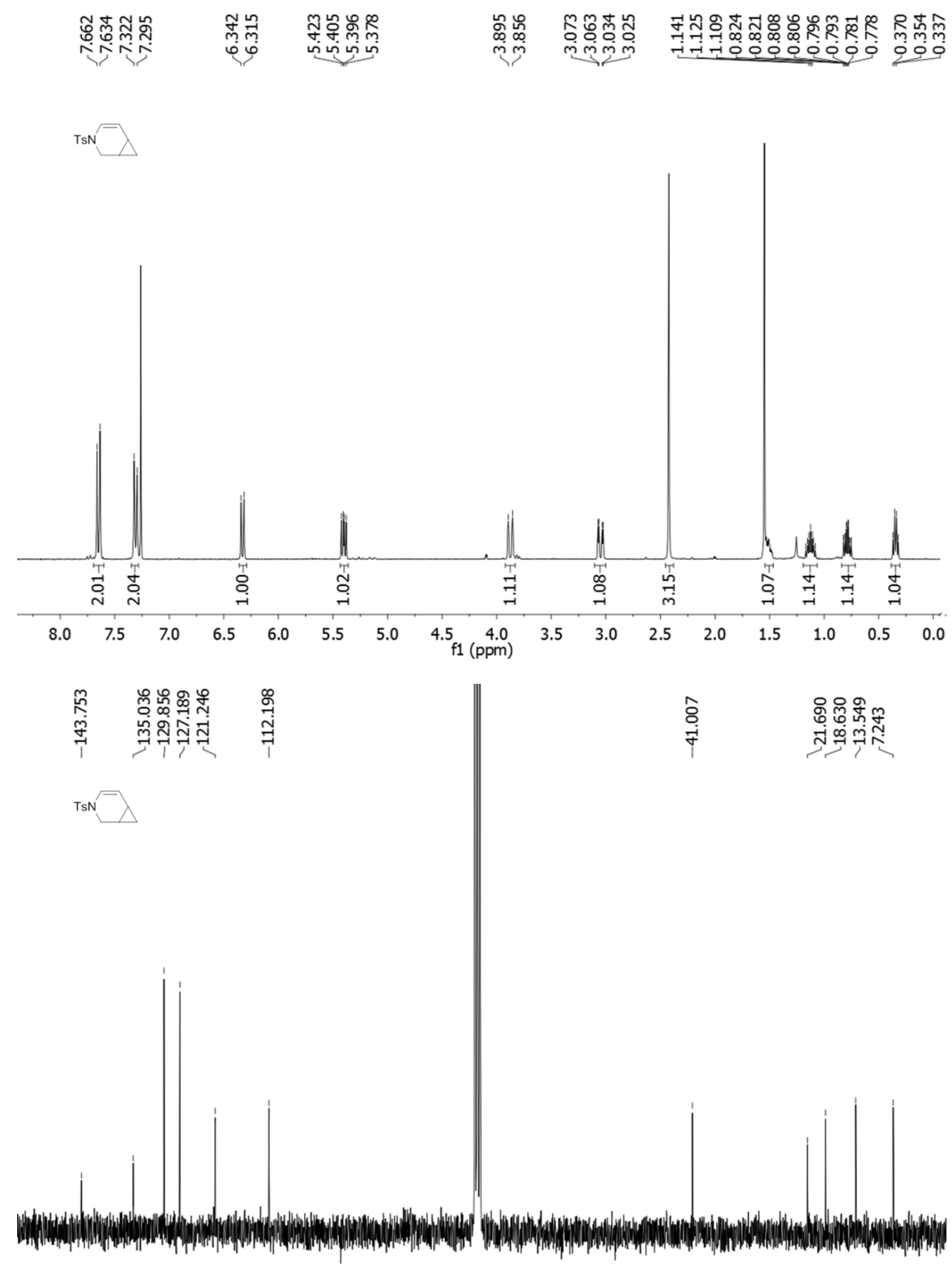

\begin{tabular}{|c|c|c|c|c|c|c|c|c|c|c|c|c|c|}
\hline 15 & 140 & 130 & 120 & 110 & 100 & 90 & $80{ }^{70}$ & 60 & 50 & 40 & 30 & 20 & 10 \\
\hline
\end{tabular}
S- 64 - 


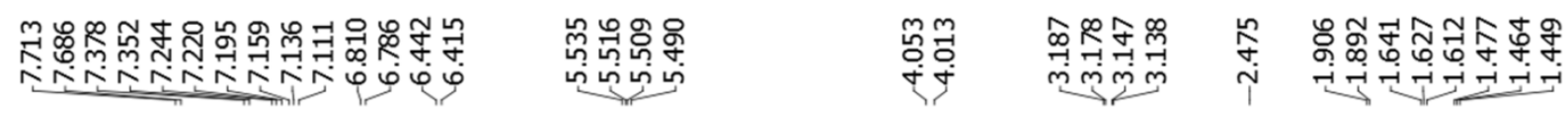
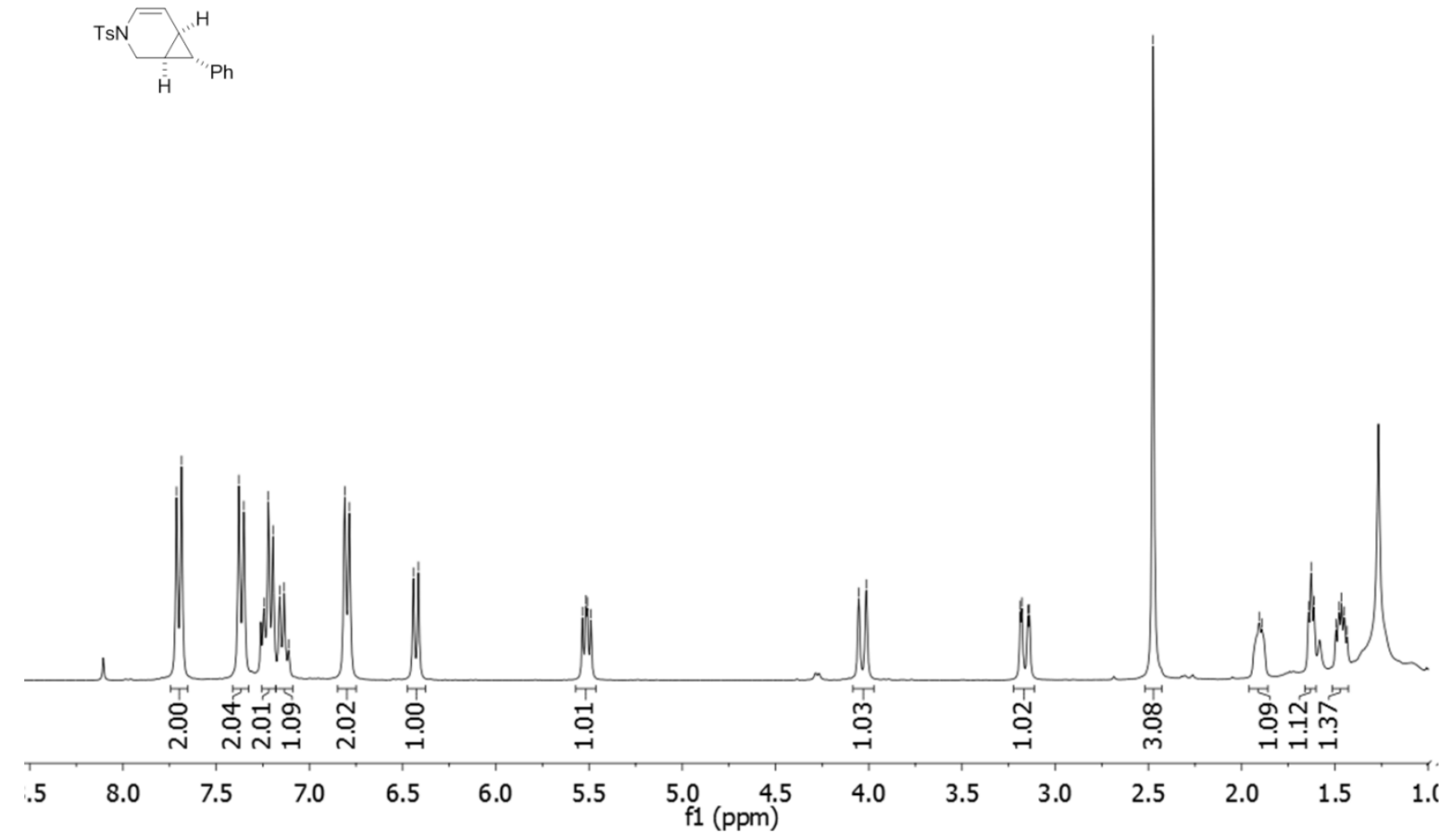

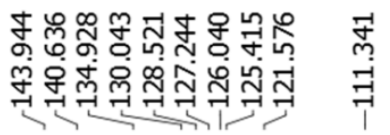

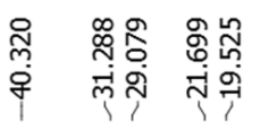
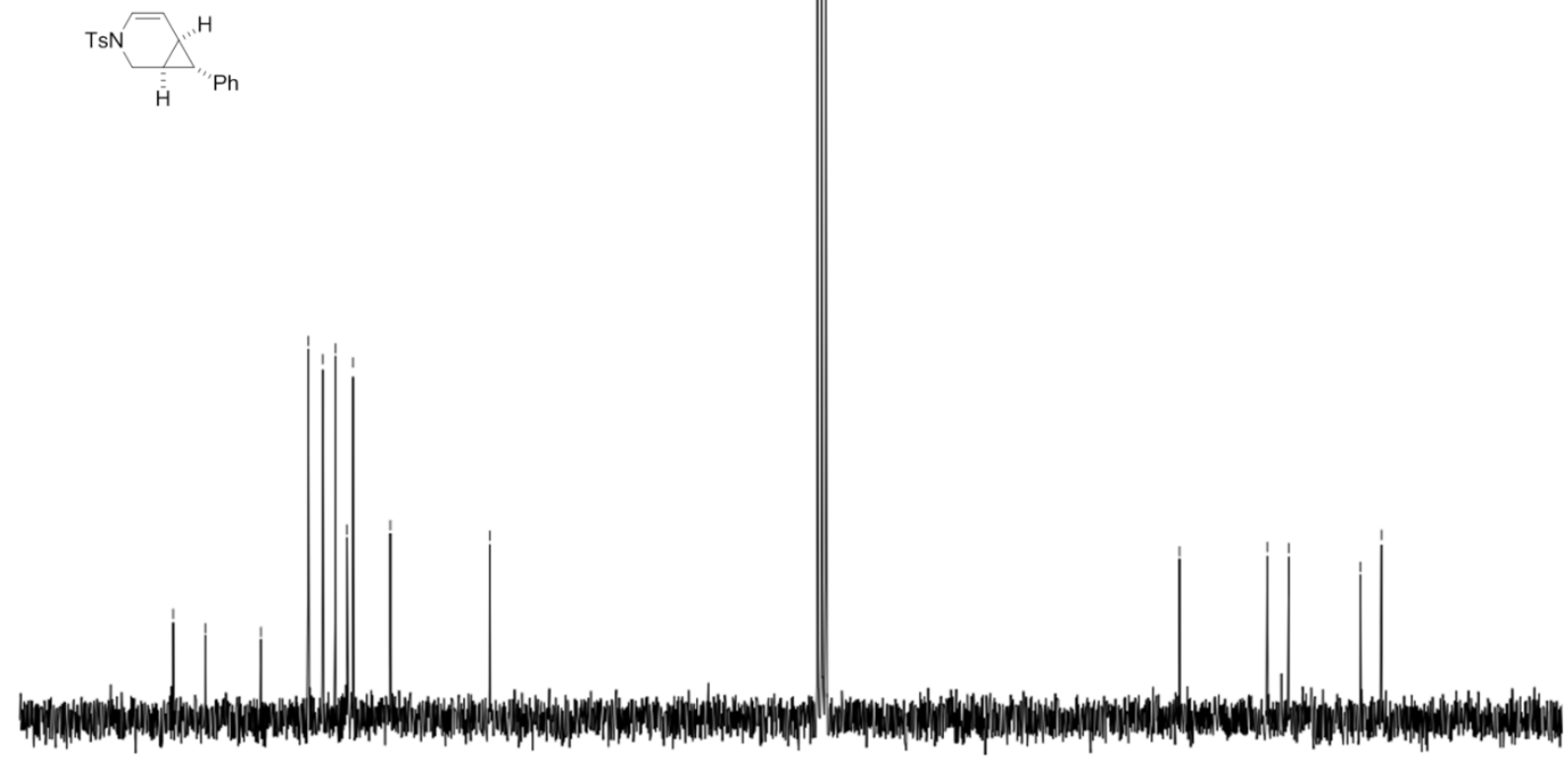

$\begin{array}{lllll}150 & 140-130-120 \quad 110-100\end{array}$

80

$7060 \quad 50$

S- 65 - 

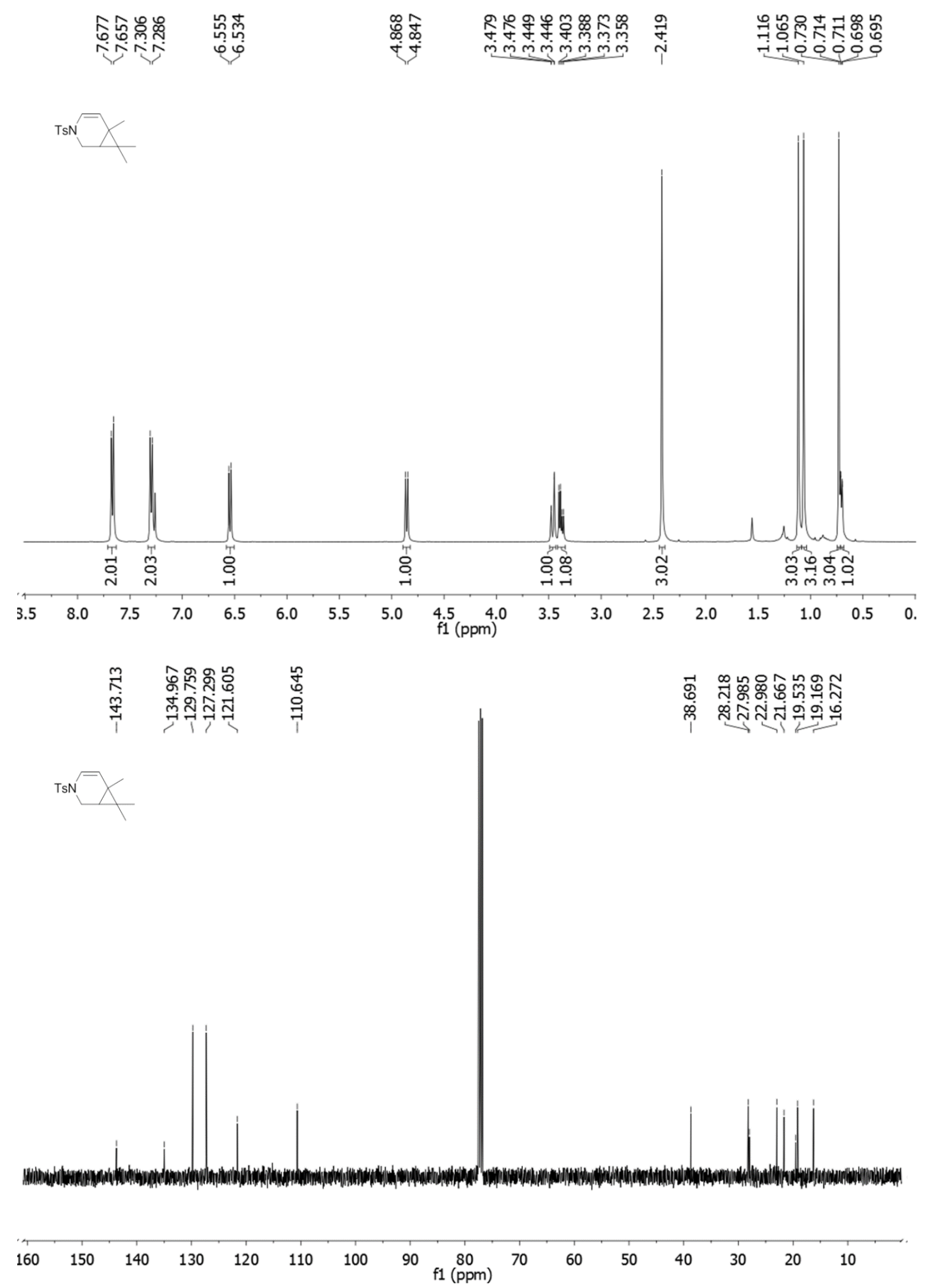

S- 66 - 


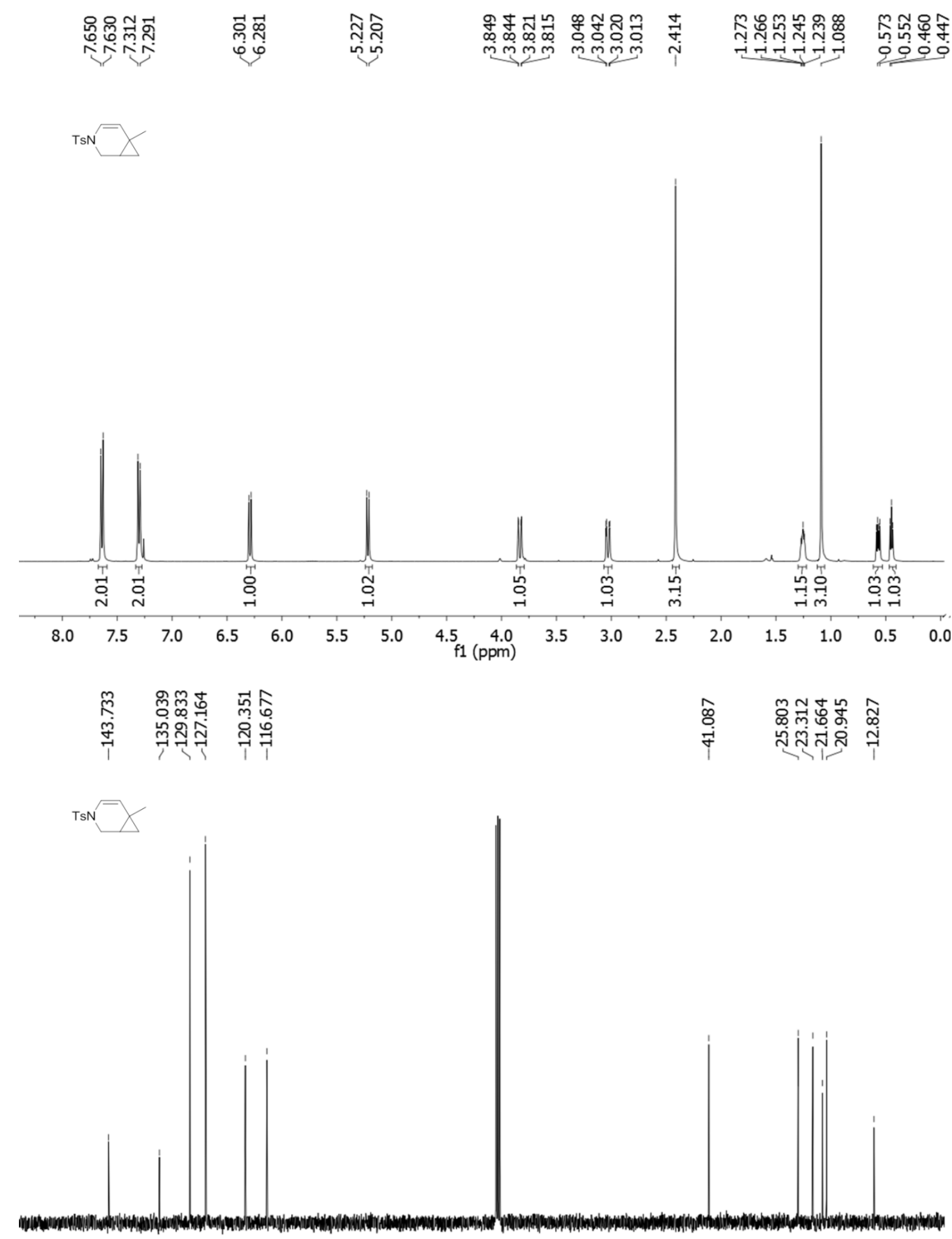

\begin{tabular}{|c|c|c|c|c|c|c|c|c|c|c|c|c|c|c|}
\hline 150 & 140 & 130 & 120 & 110 & 100 & 90 & $\begin{array}{c}80 \\
\mathrm{f1}(\mathrm{ppm})\end{array}$ & 70 & 60 & 50 & 40 & 30 & 20 & 10 \\
\hline
\end{tabular}

S- 67 - 


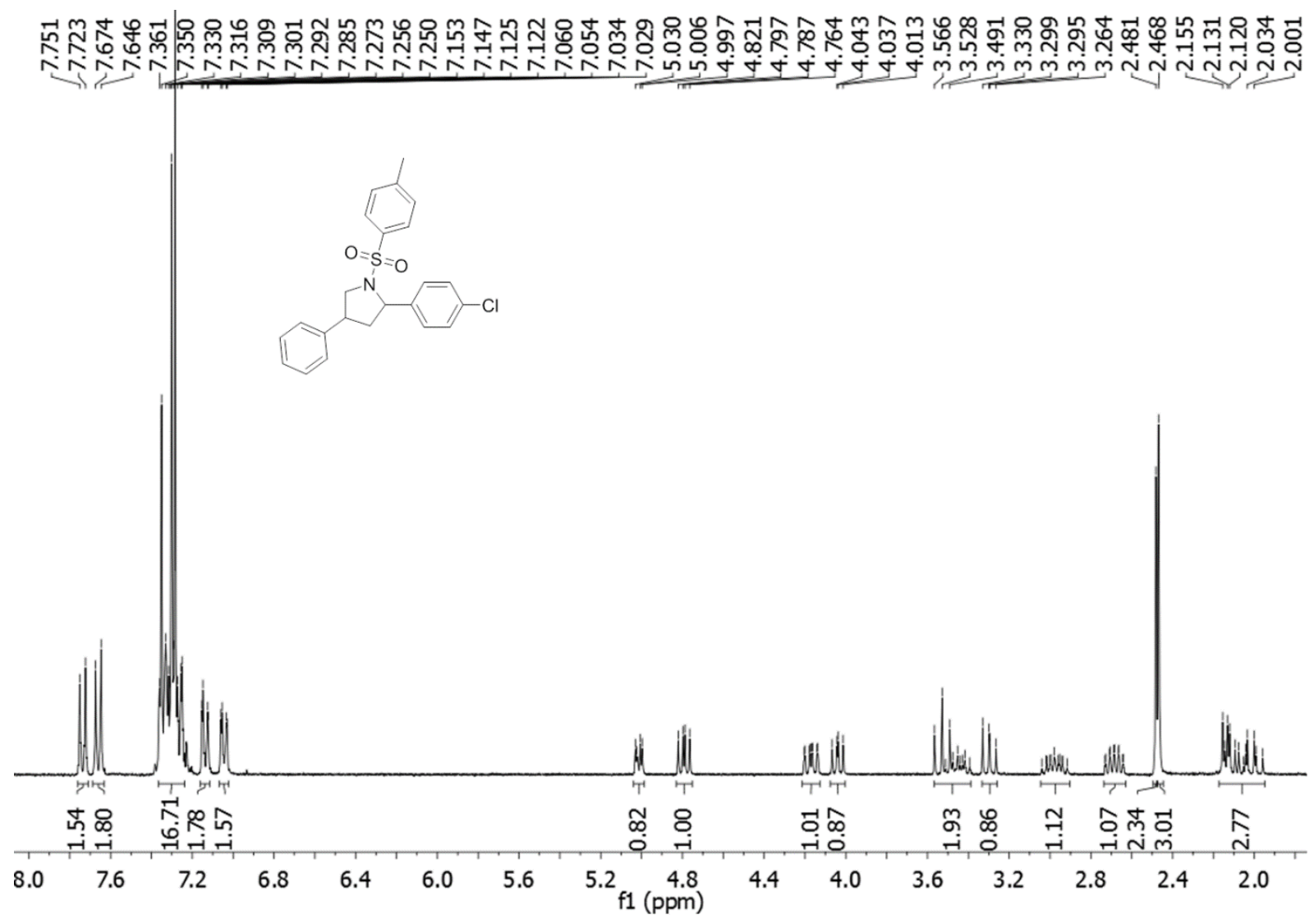

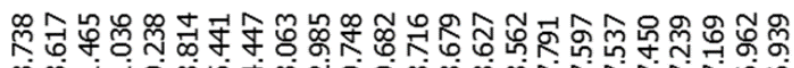

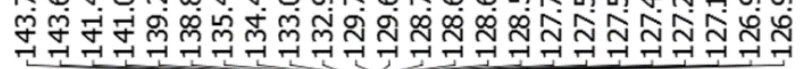
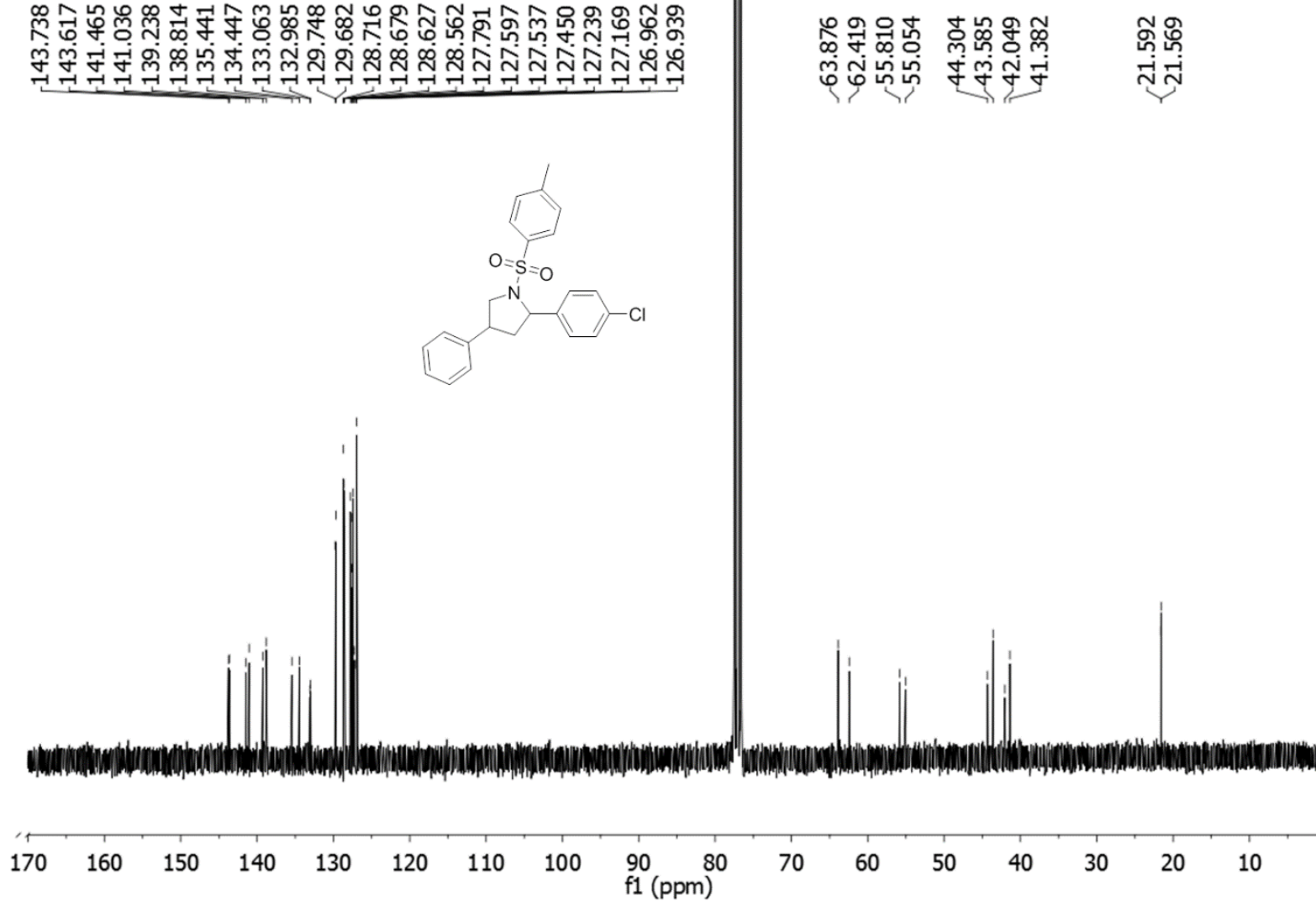


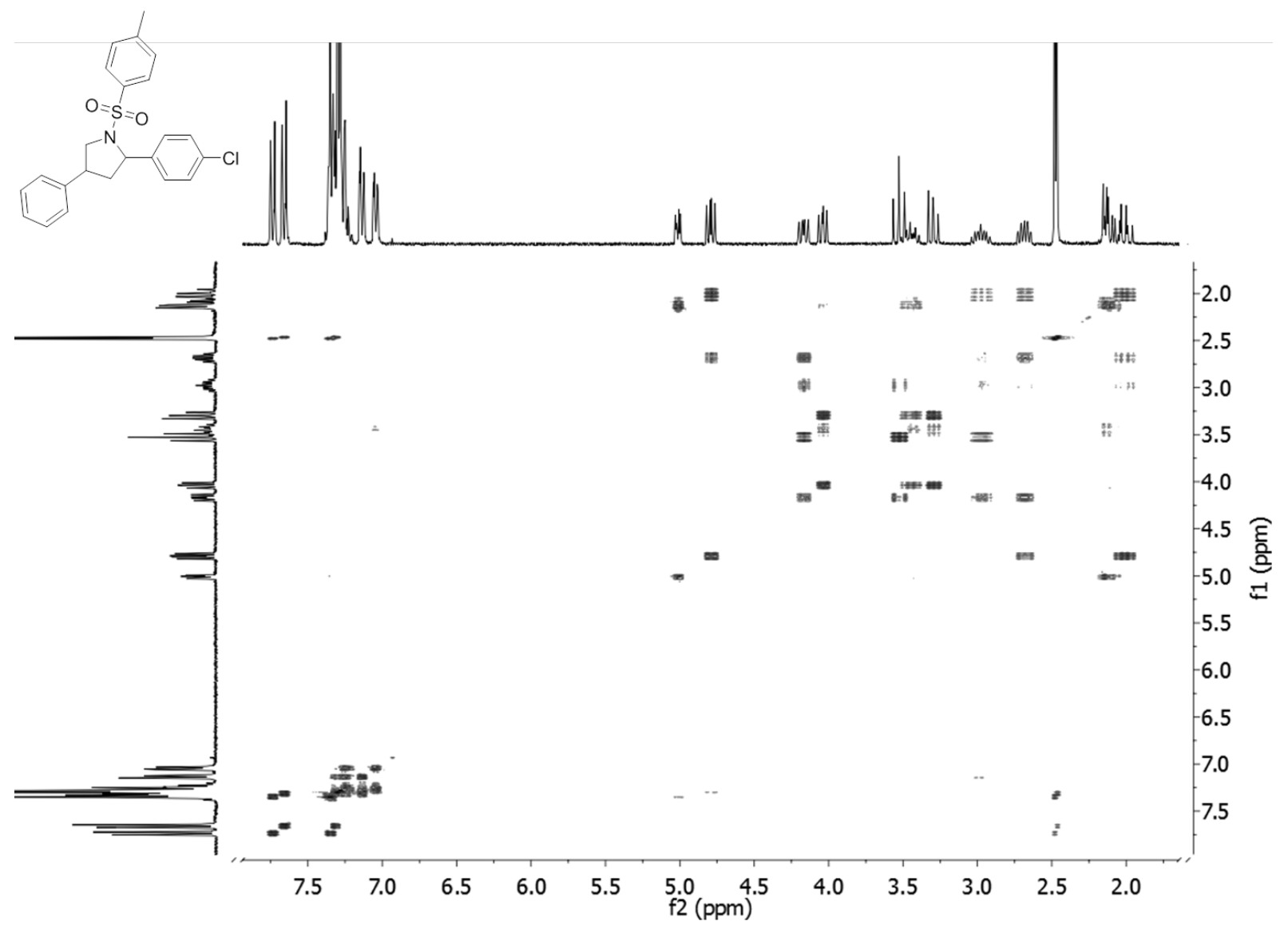



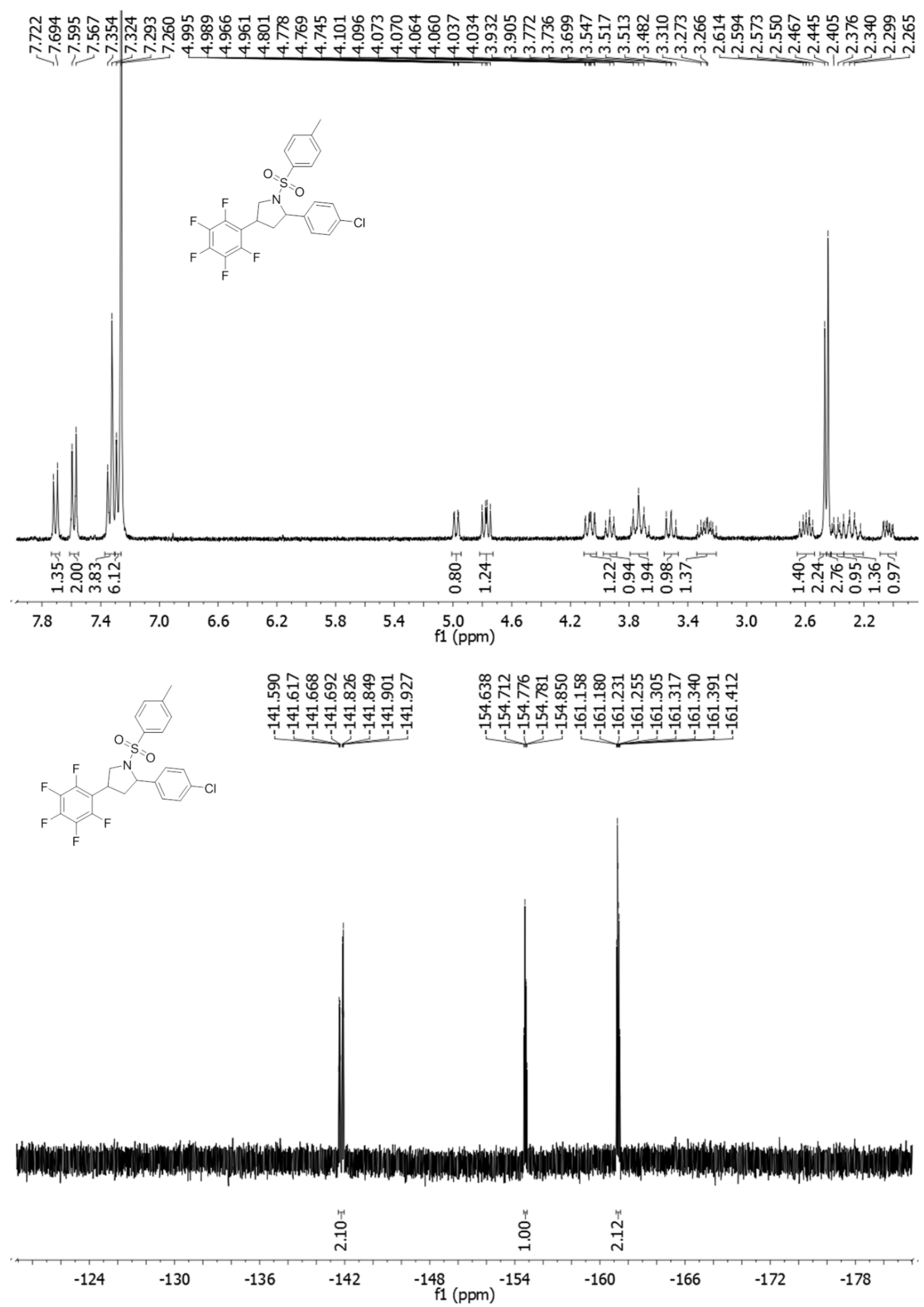

S- 70 - 


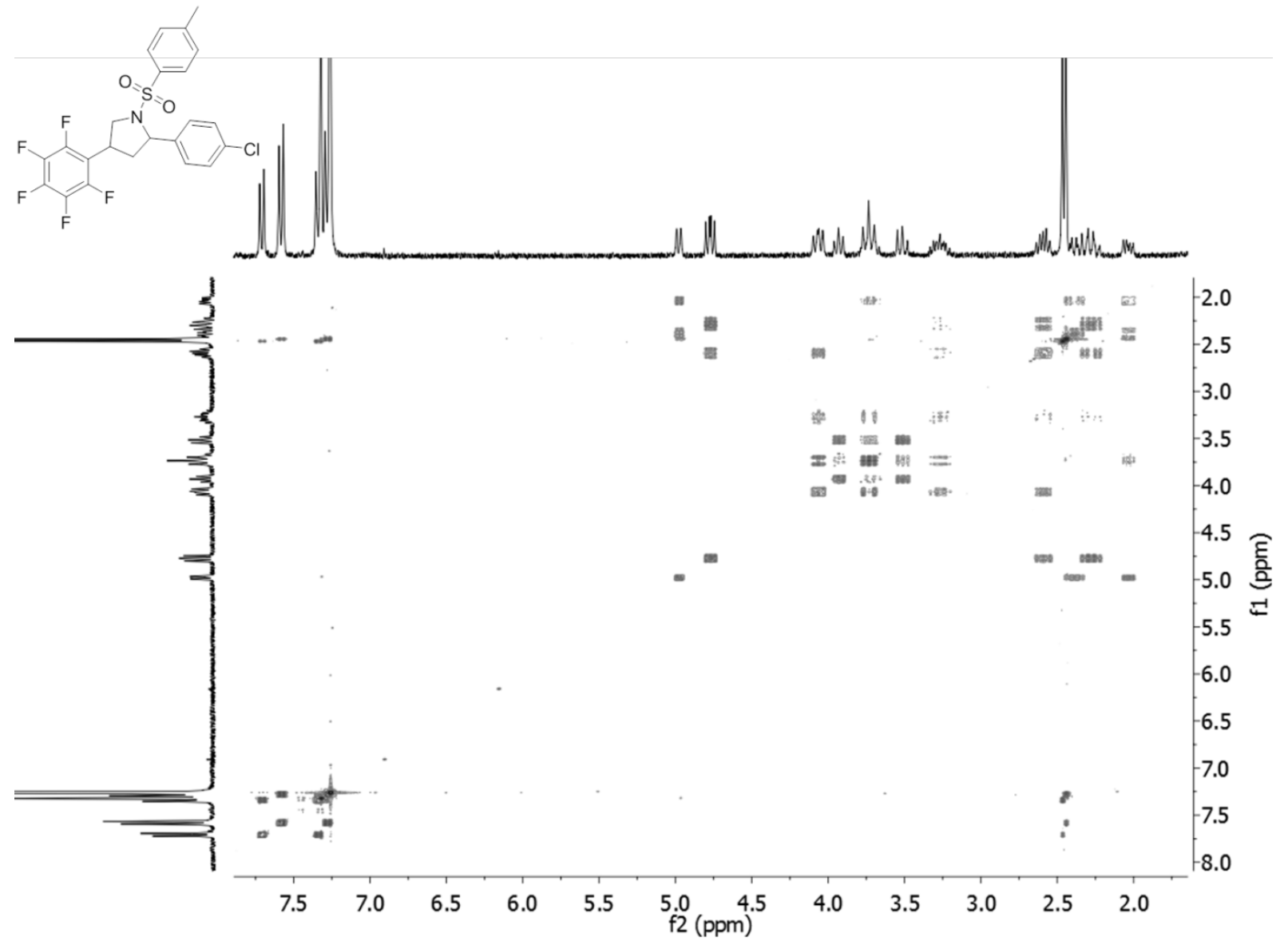




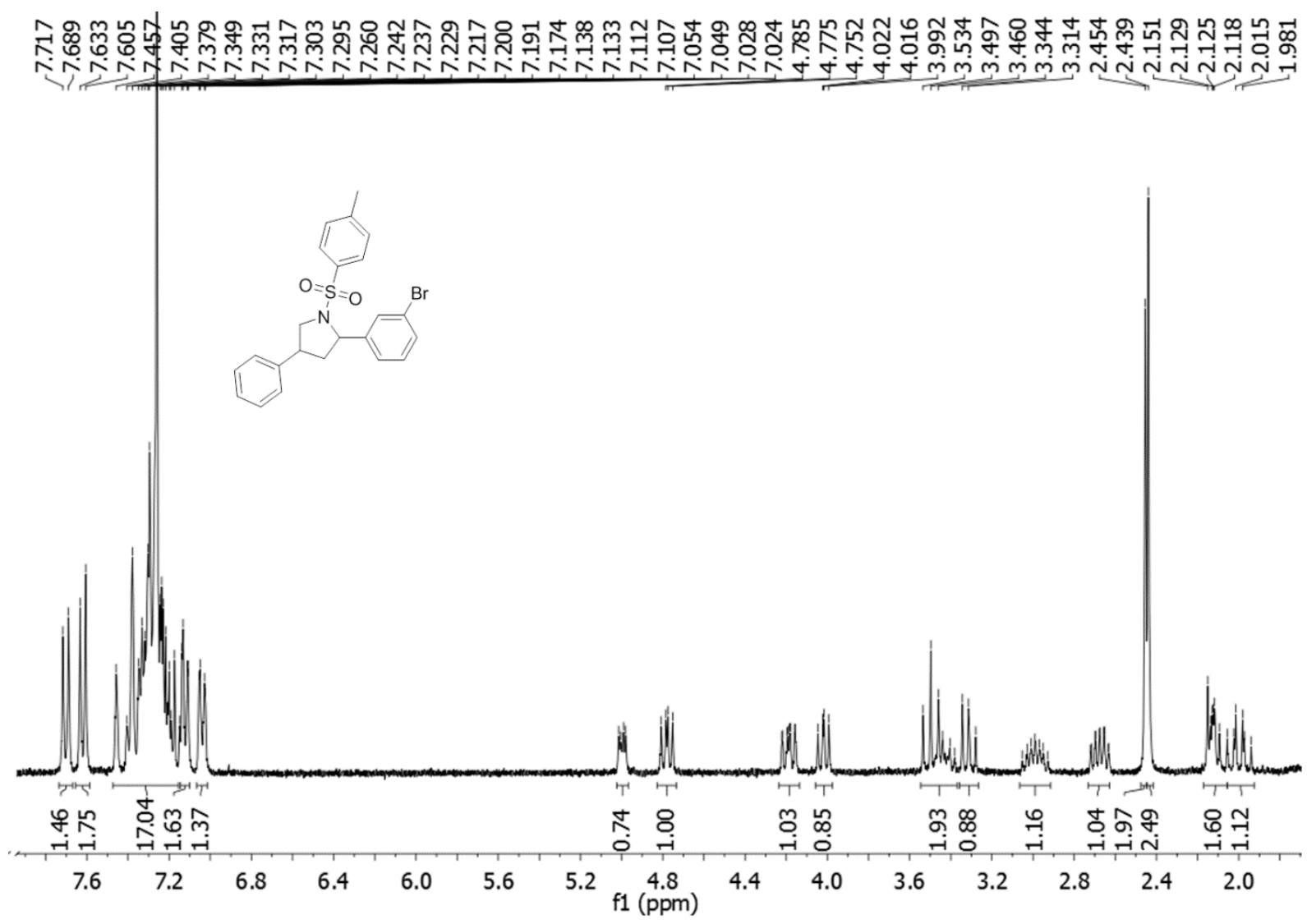

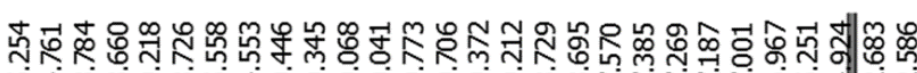

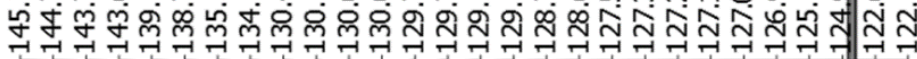
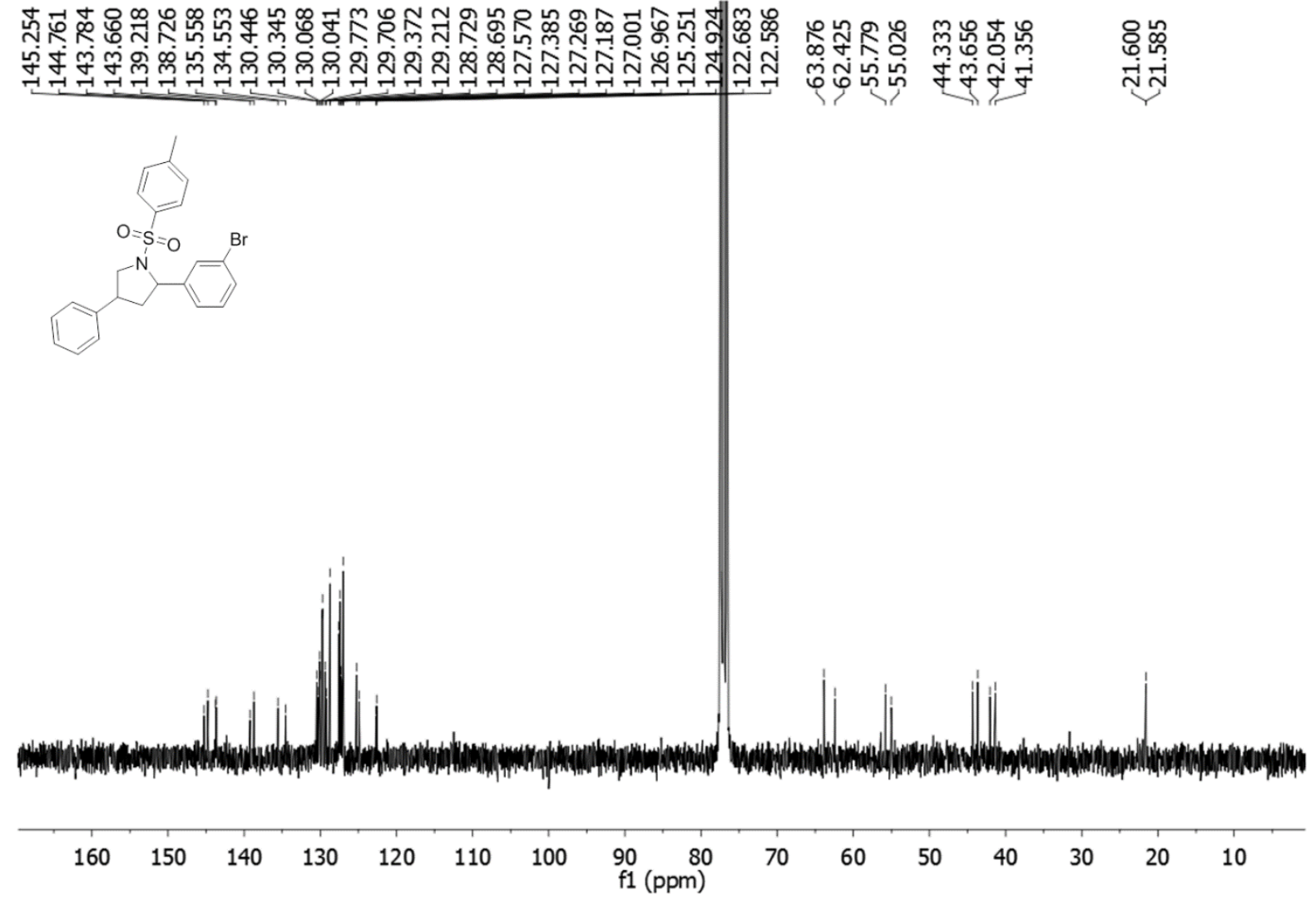


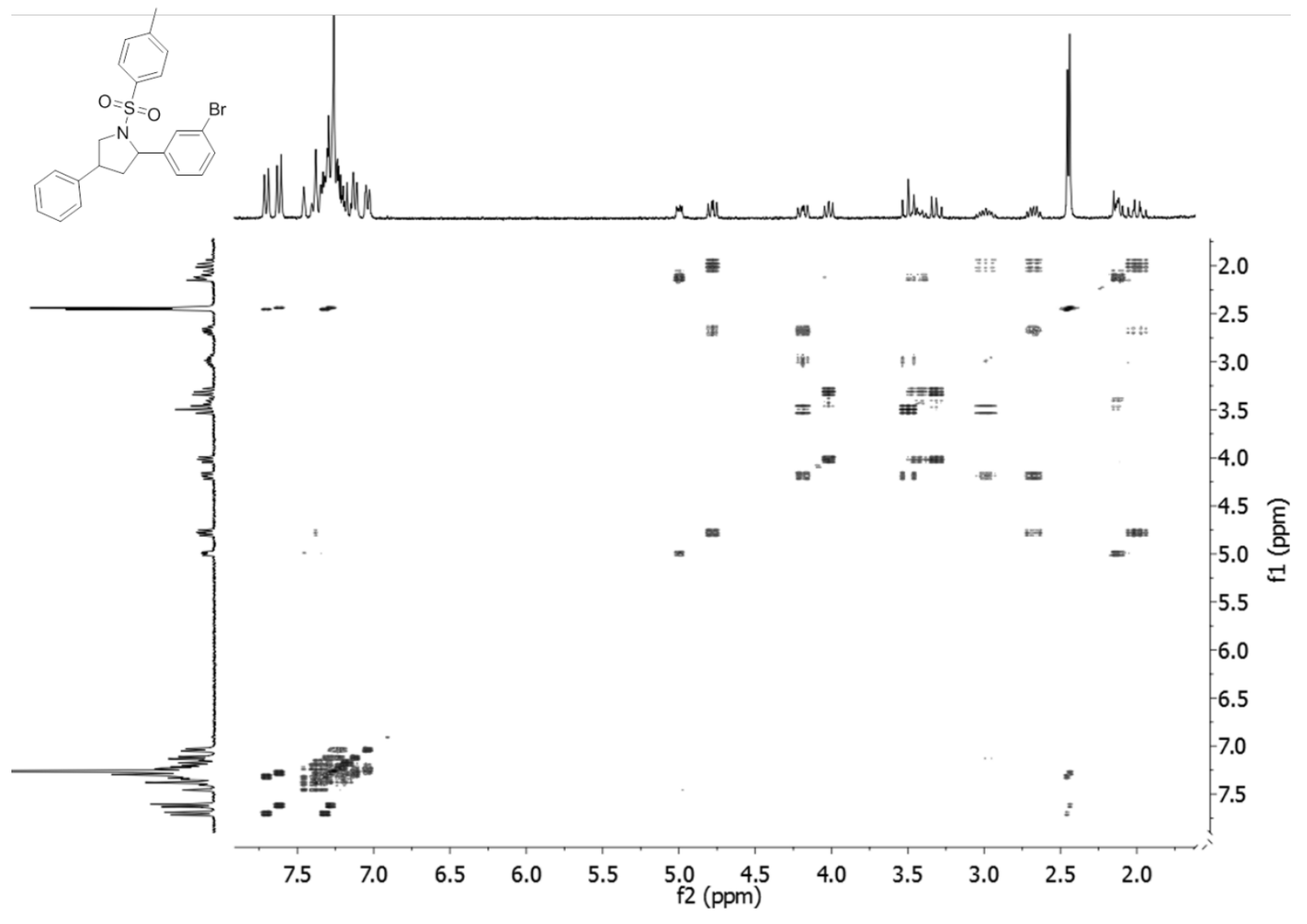

S- 73 - 


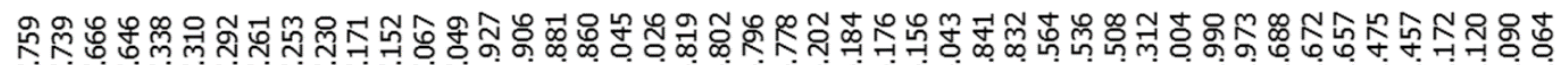

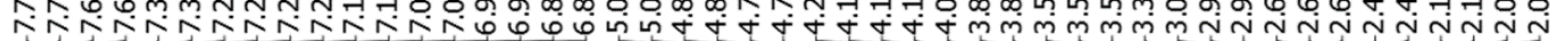
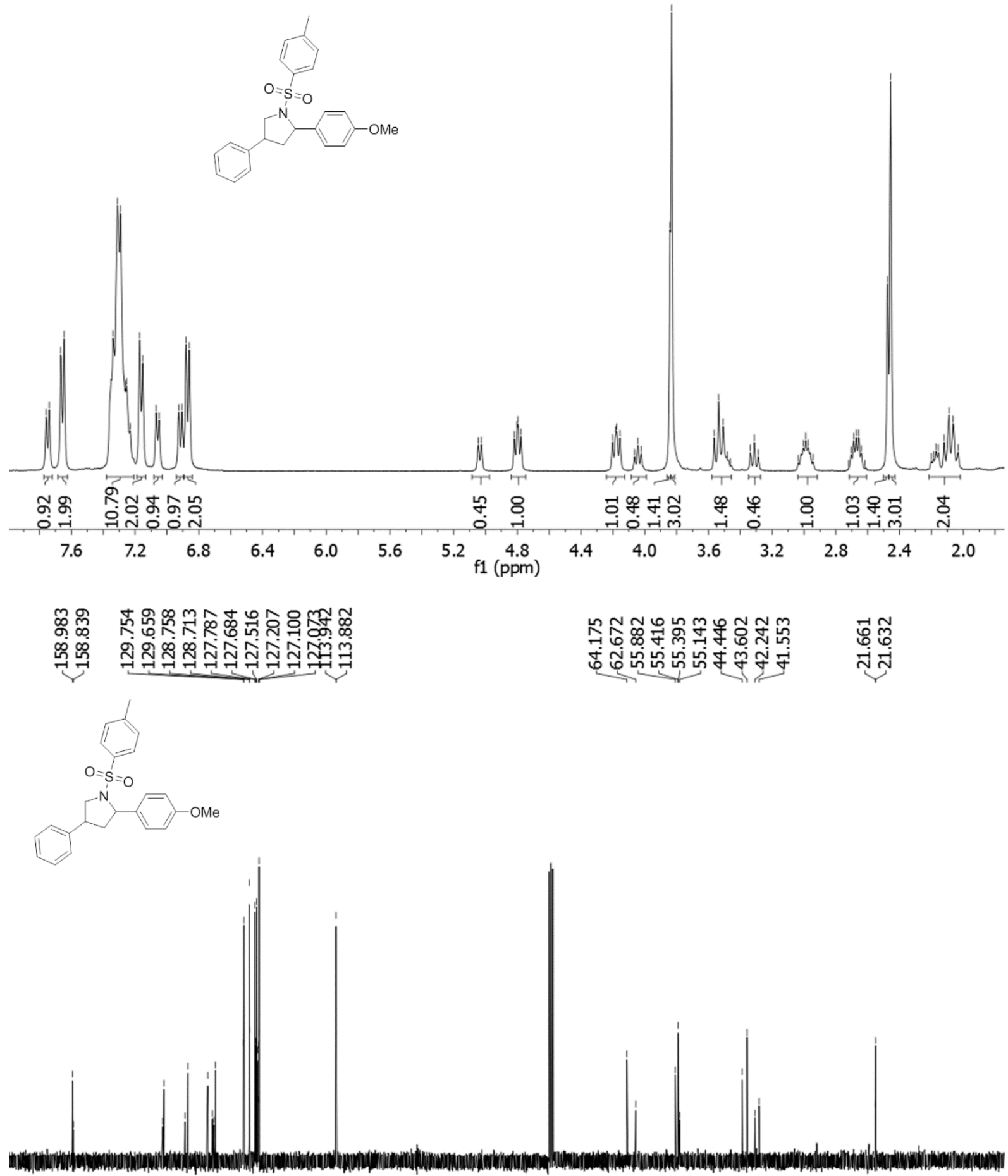

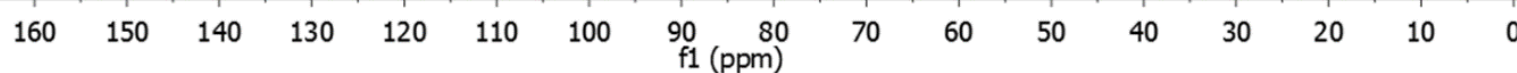




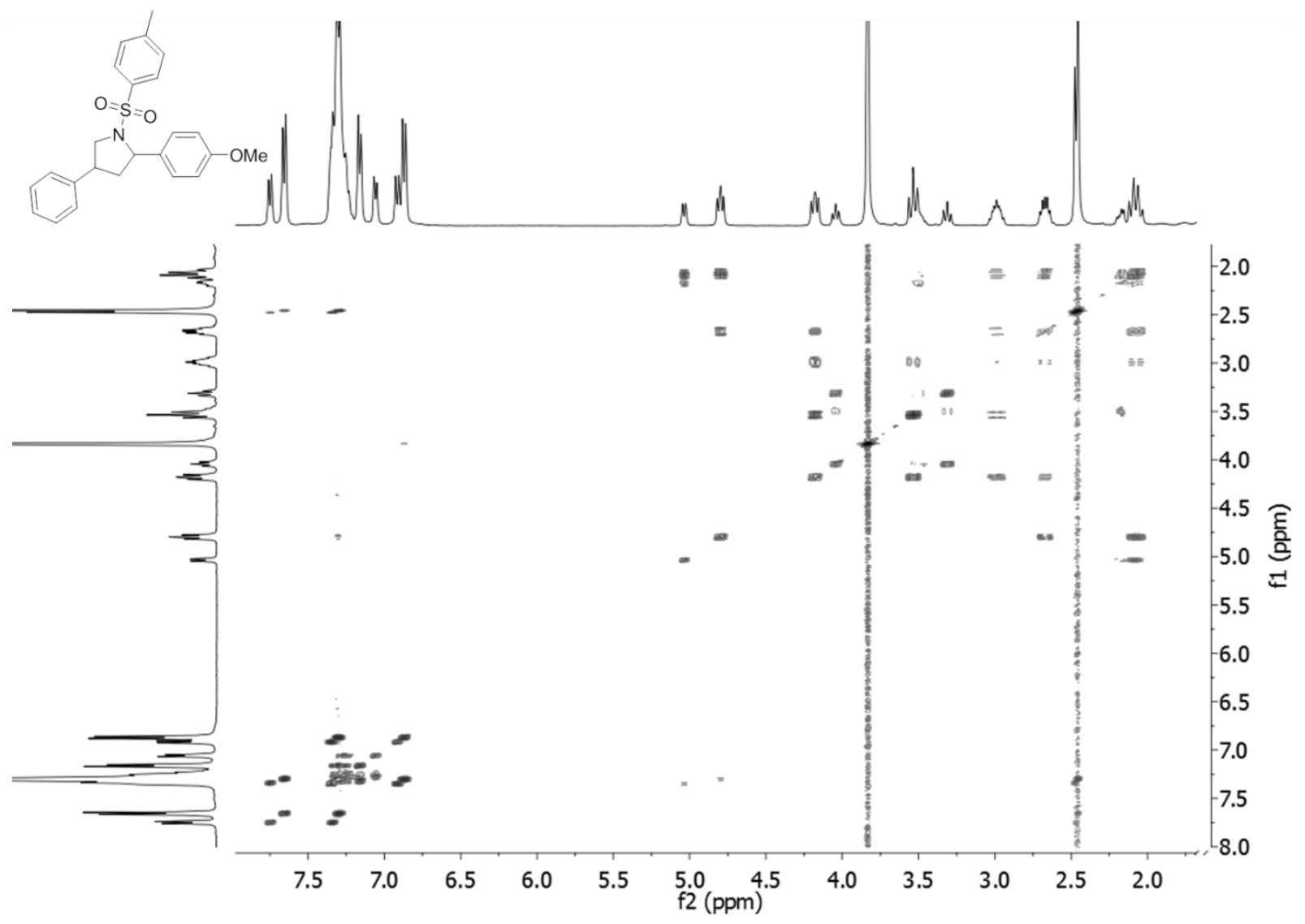

S- 75 - 


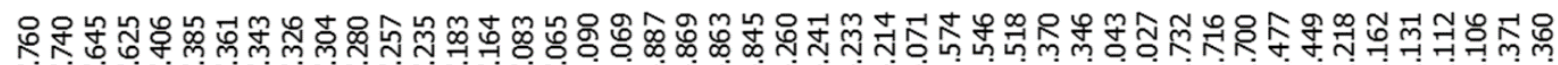

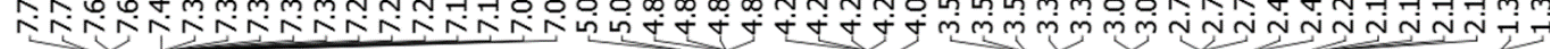
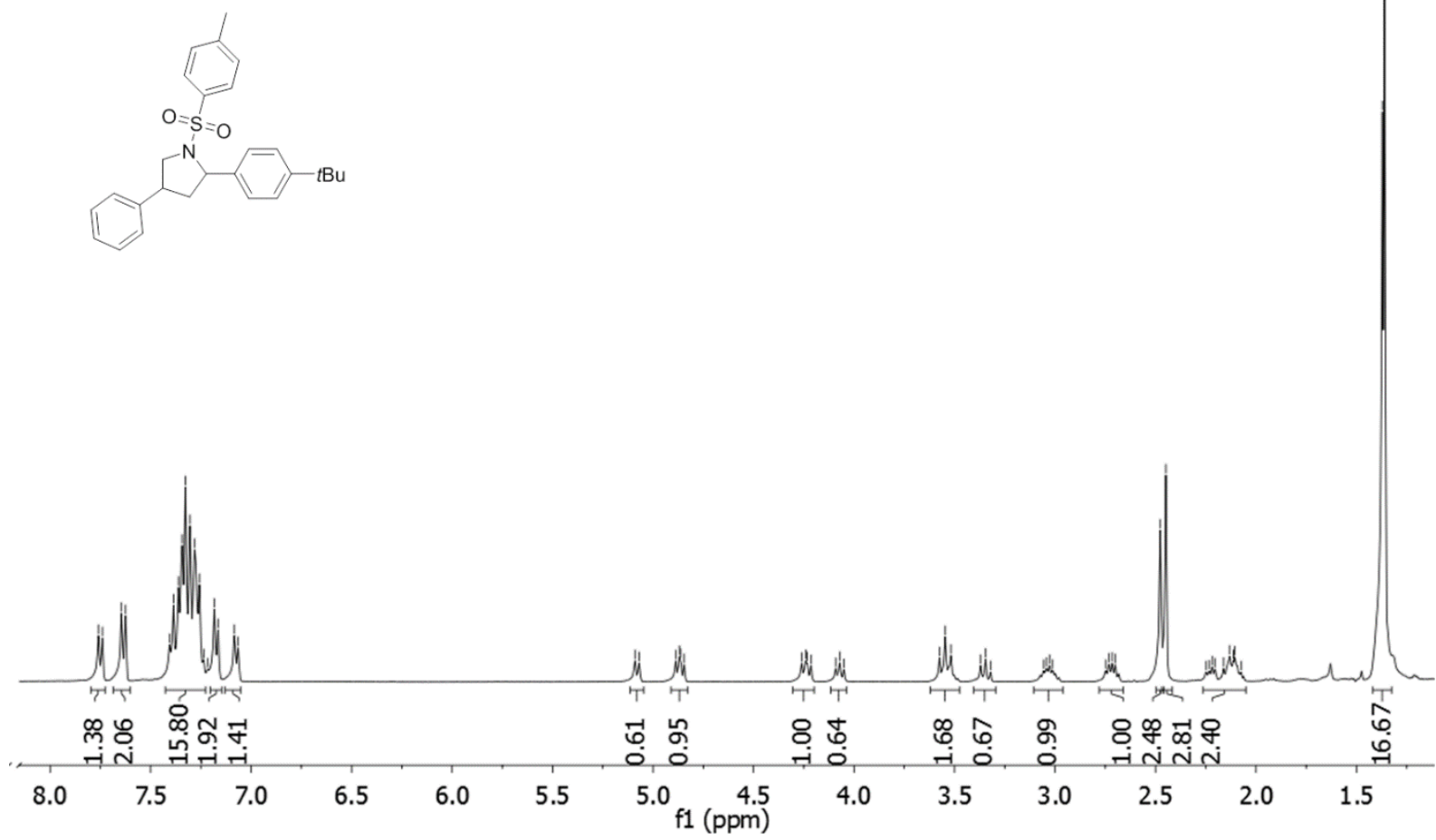

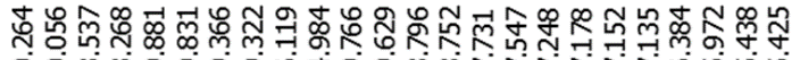

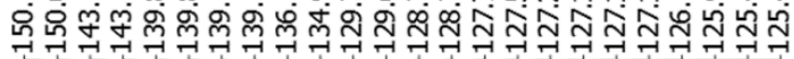

ํํㅇํㅇำ

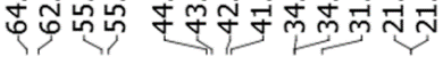
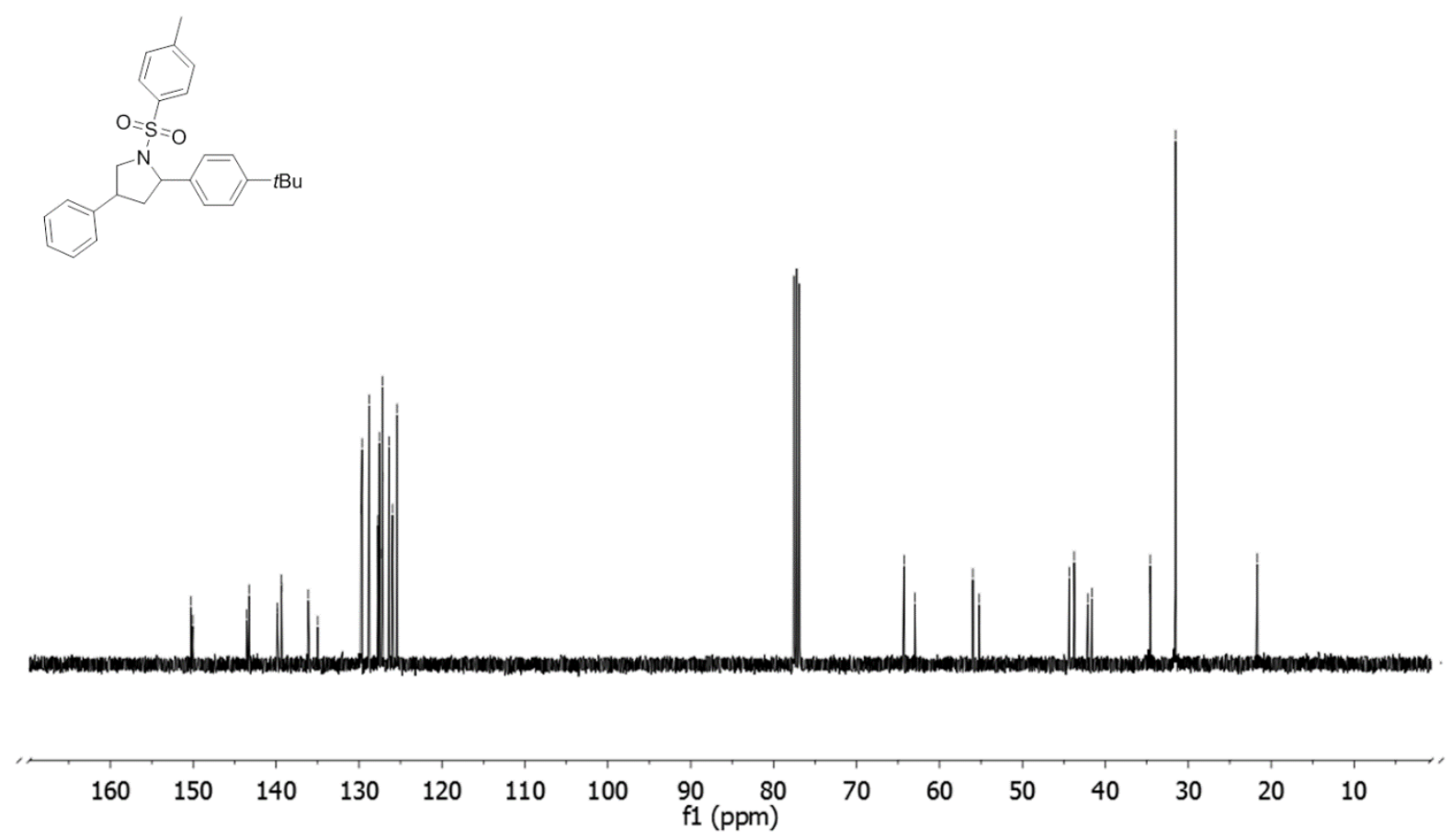


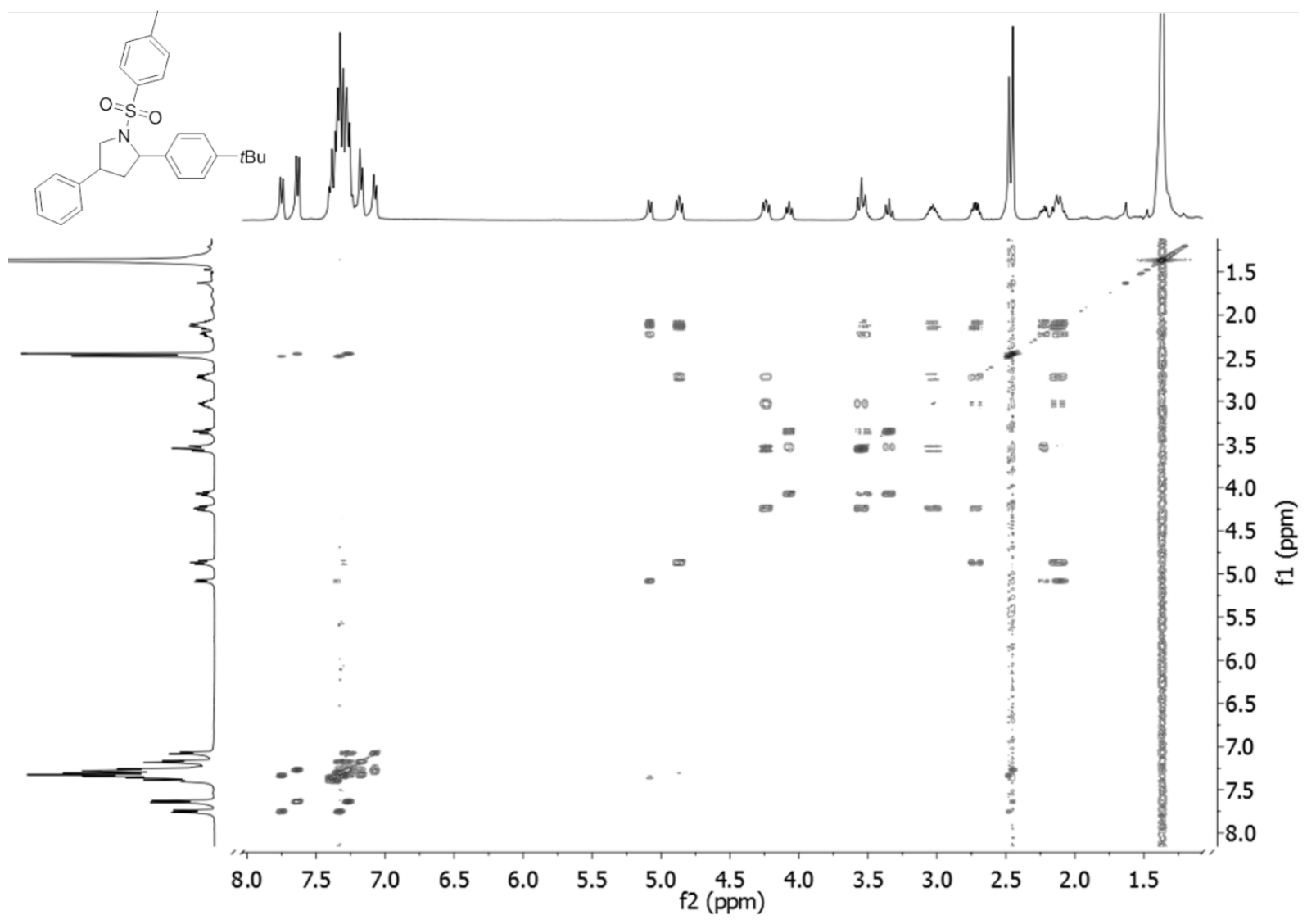




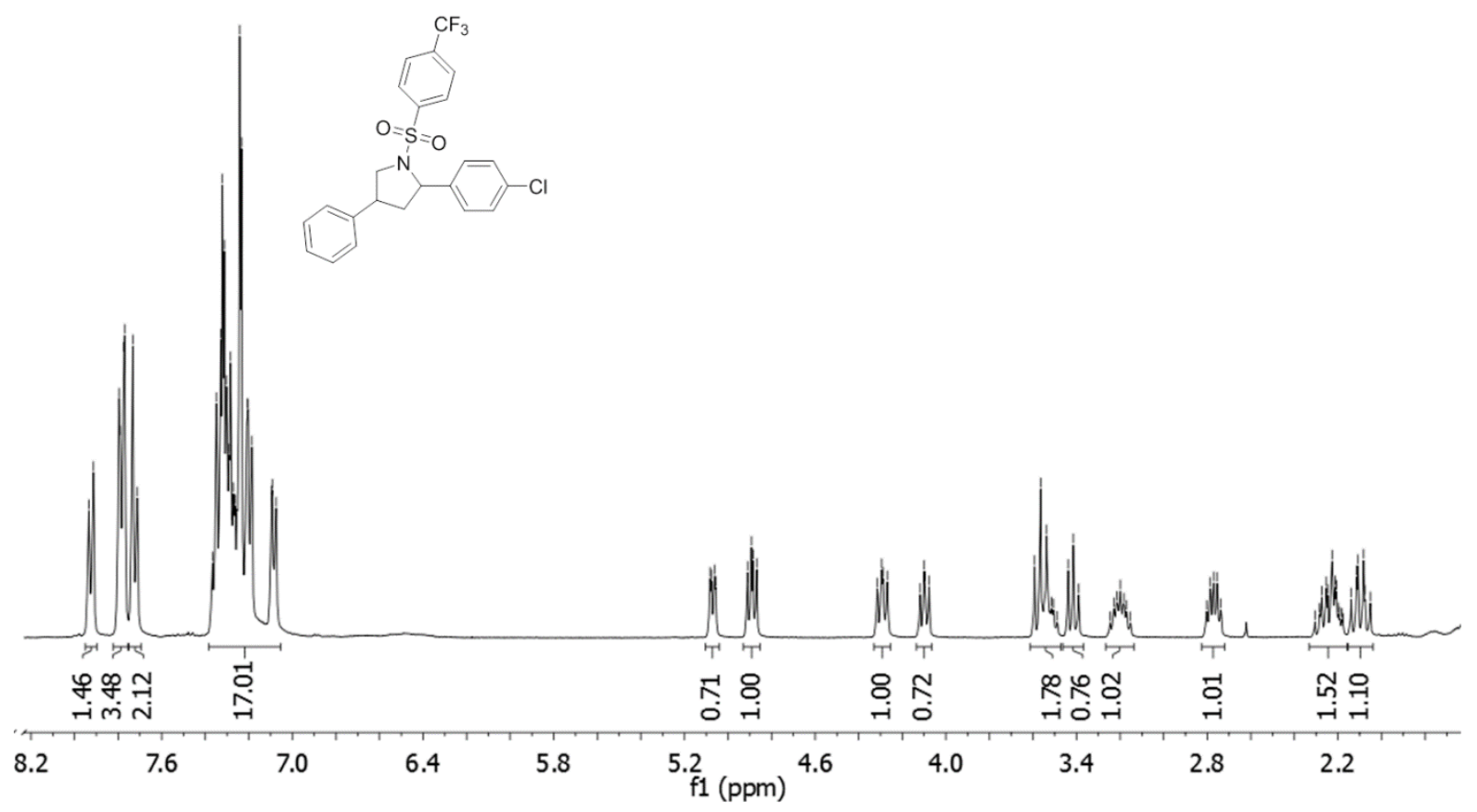

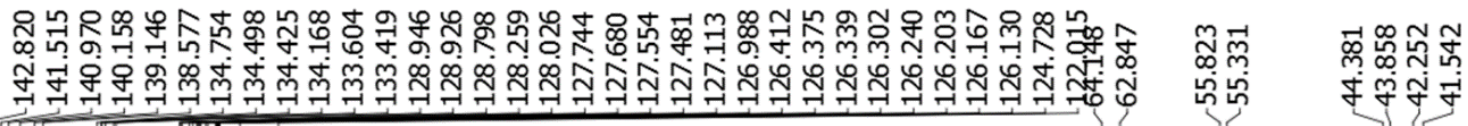

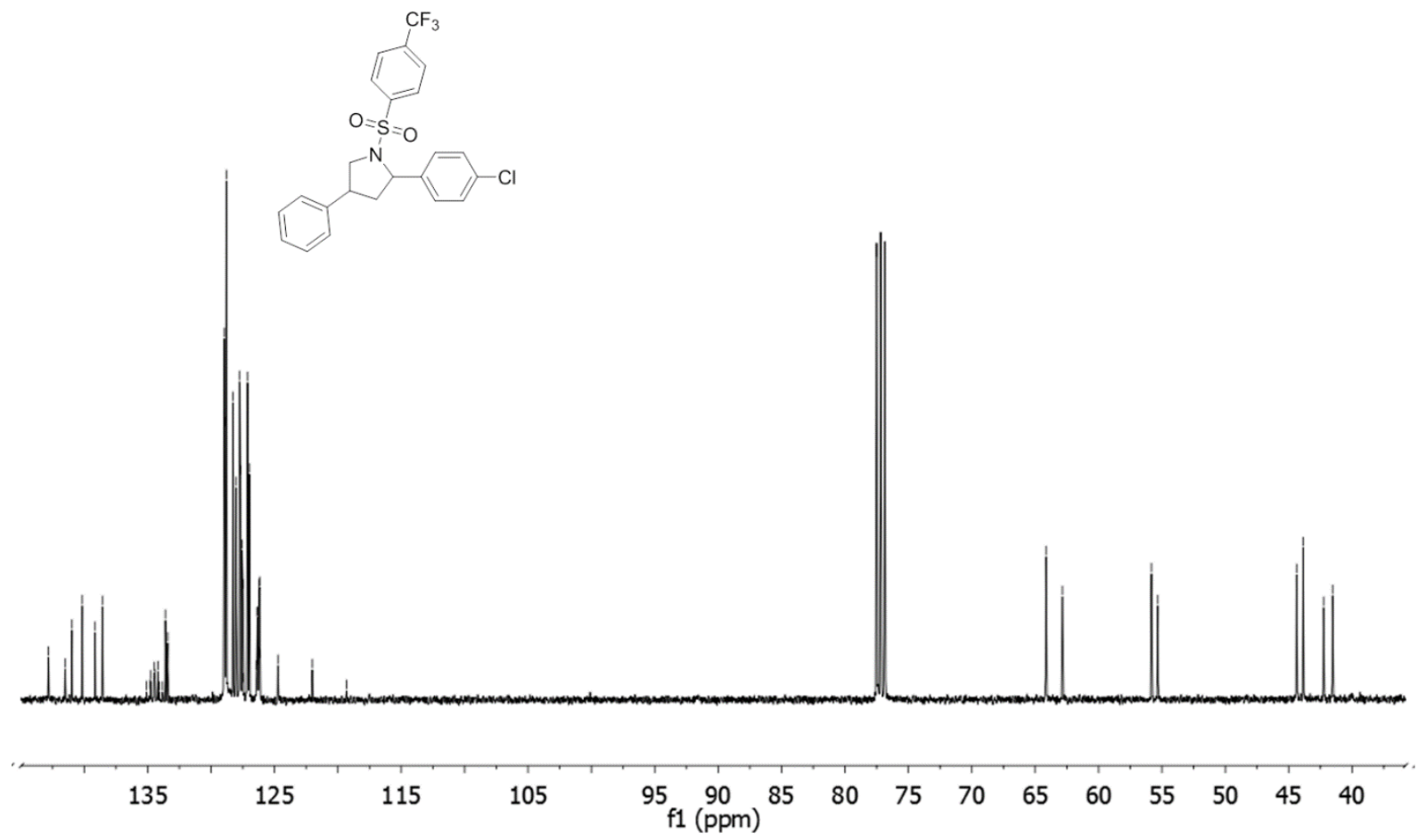




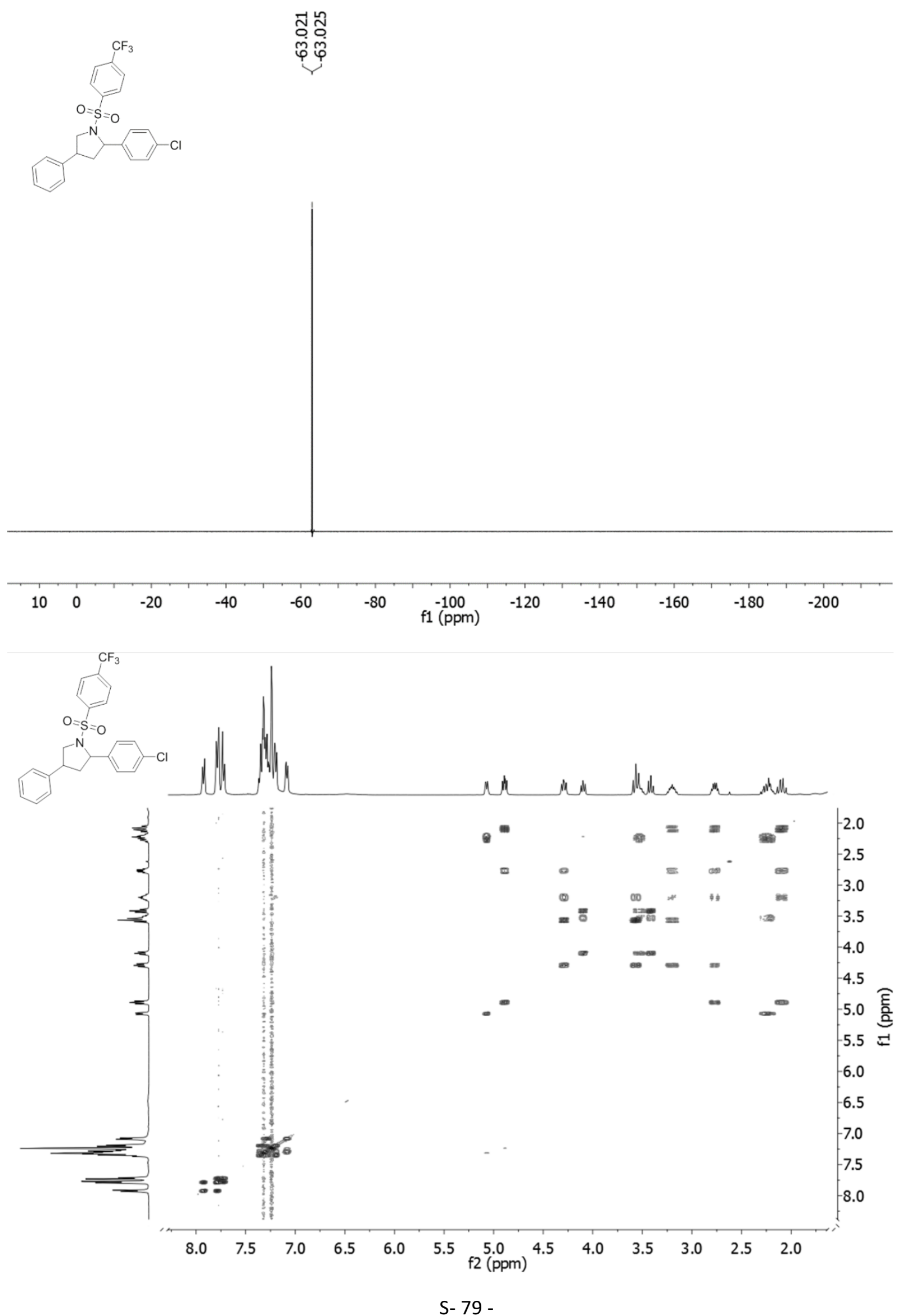




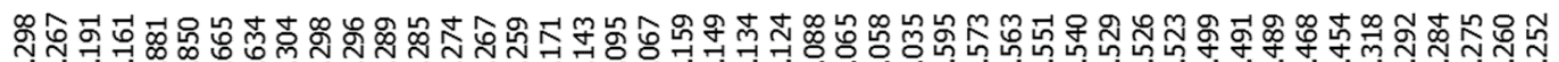

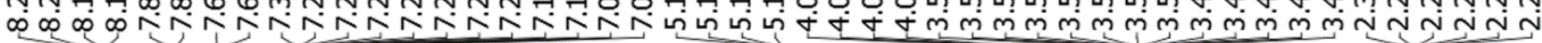

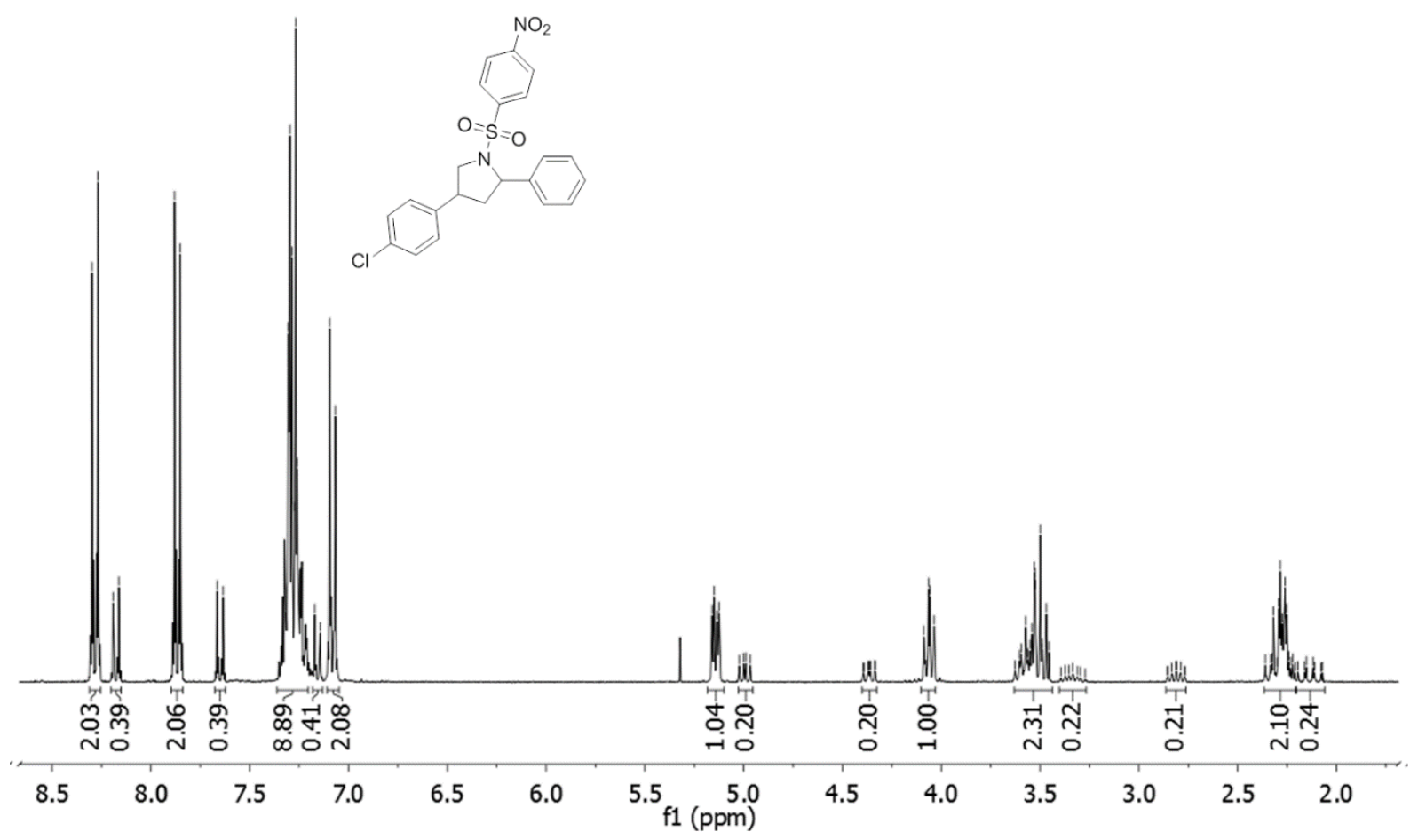

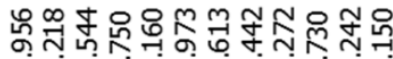

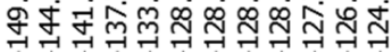
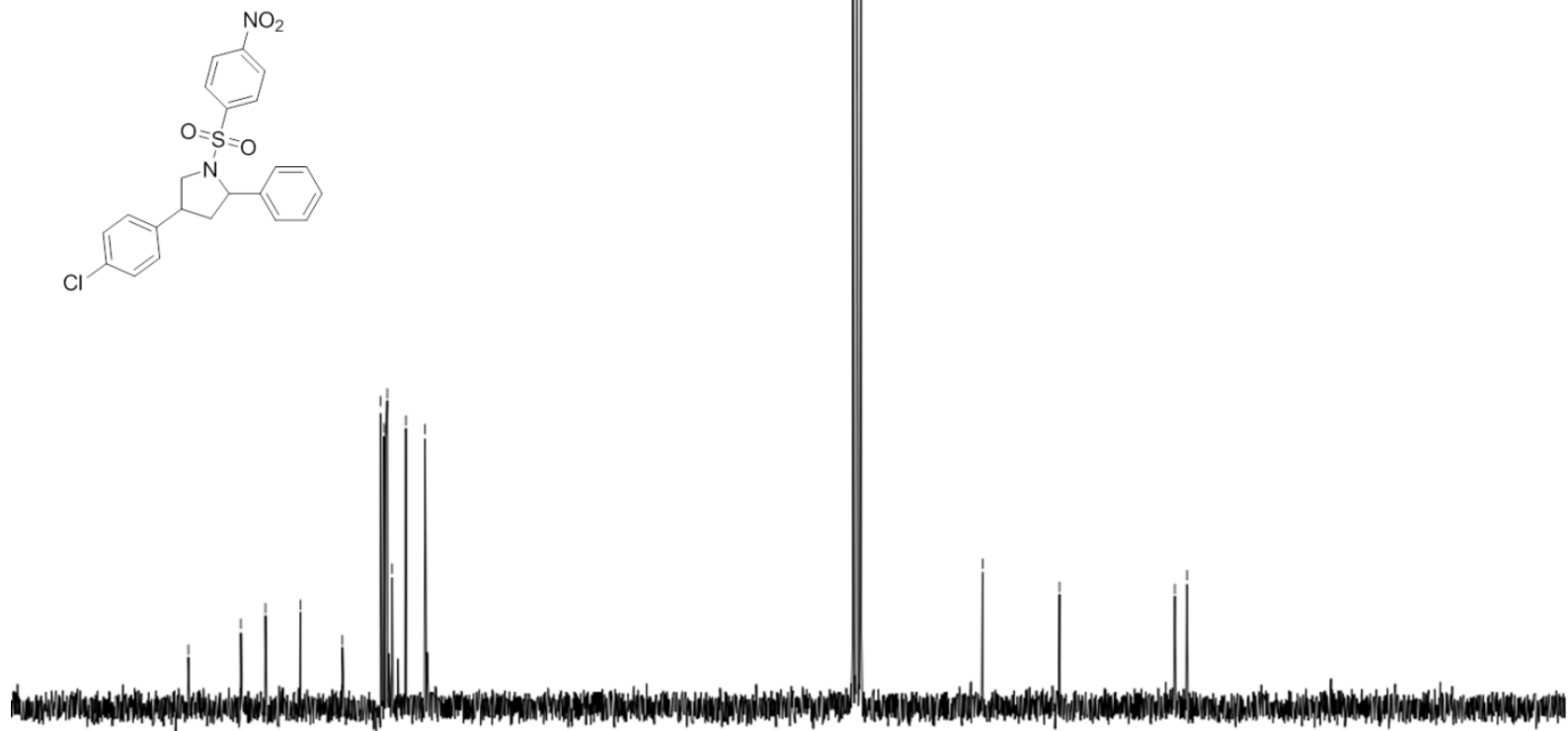

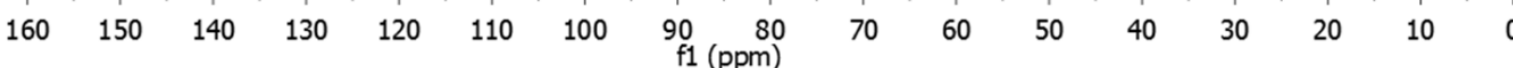




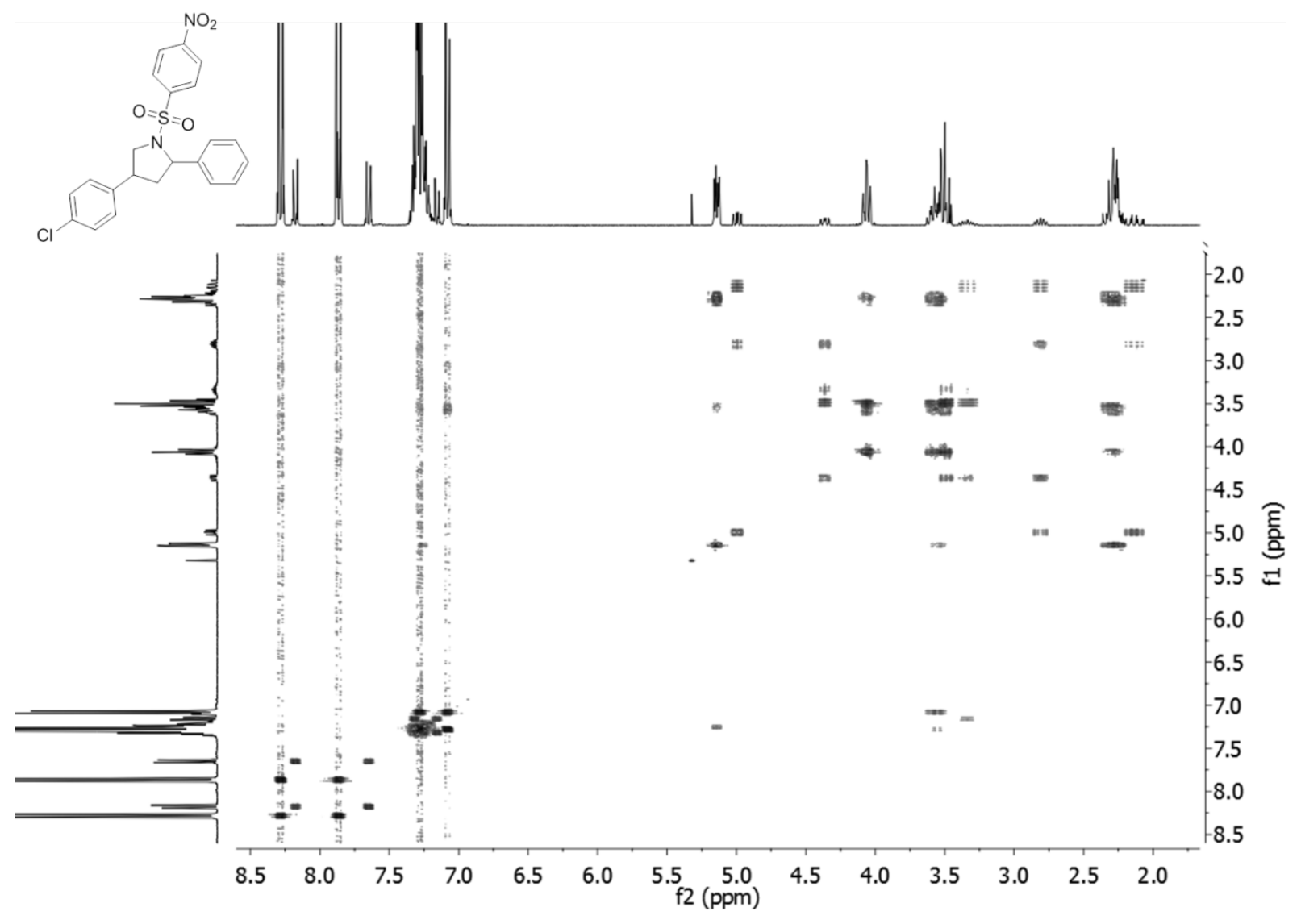




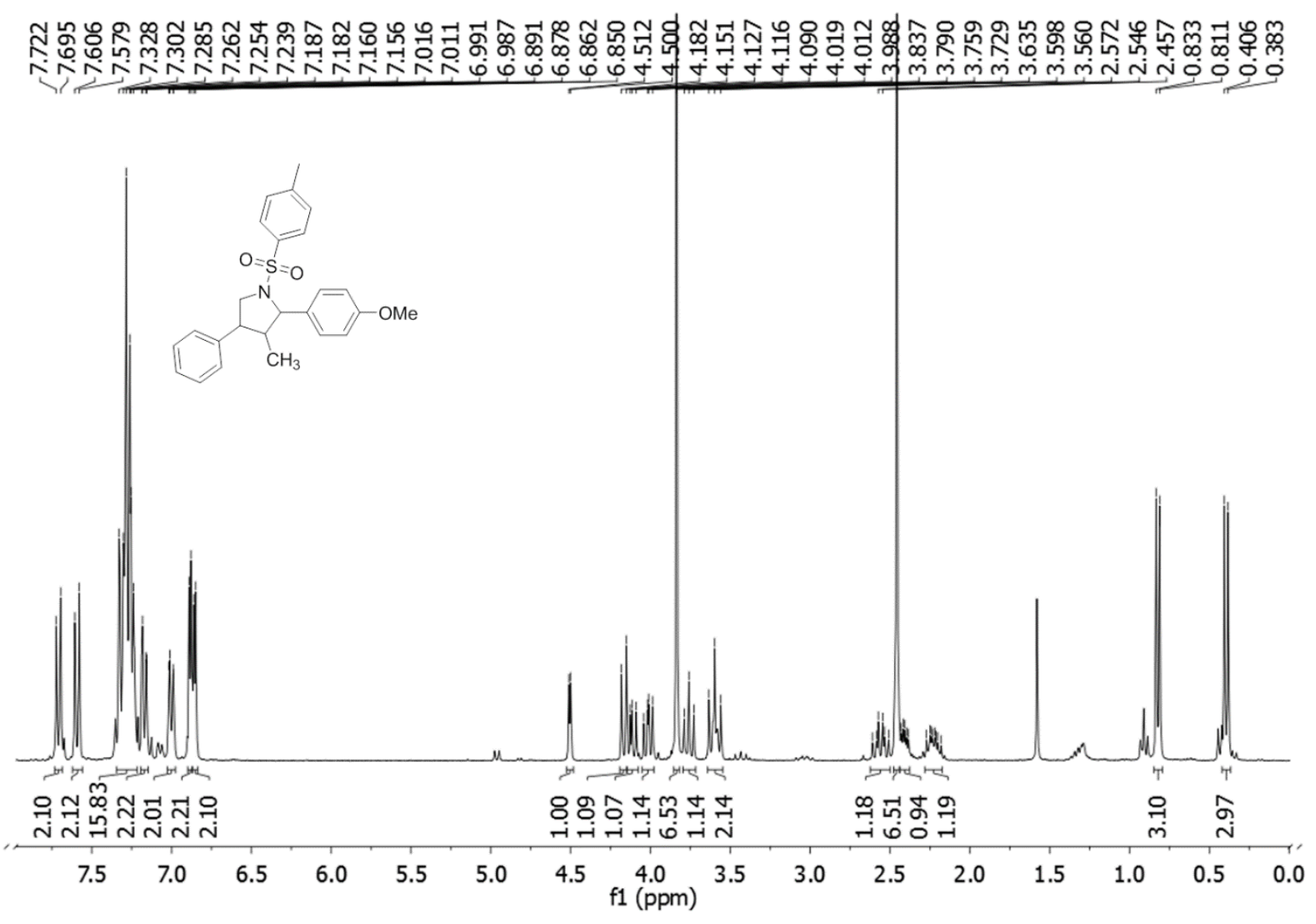

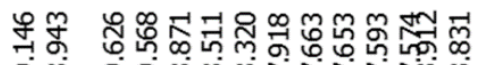

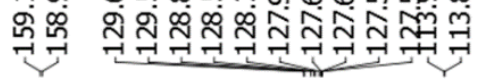

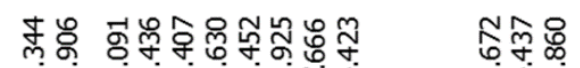

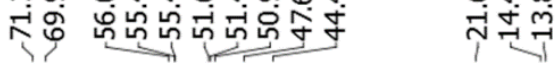
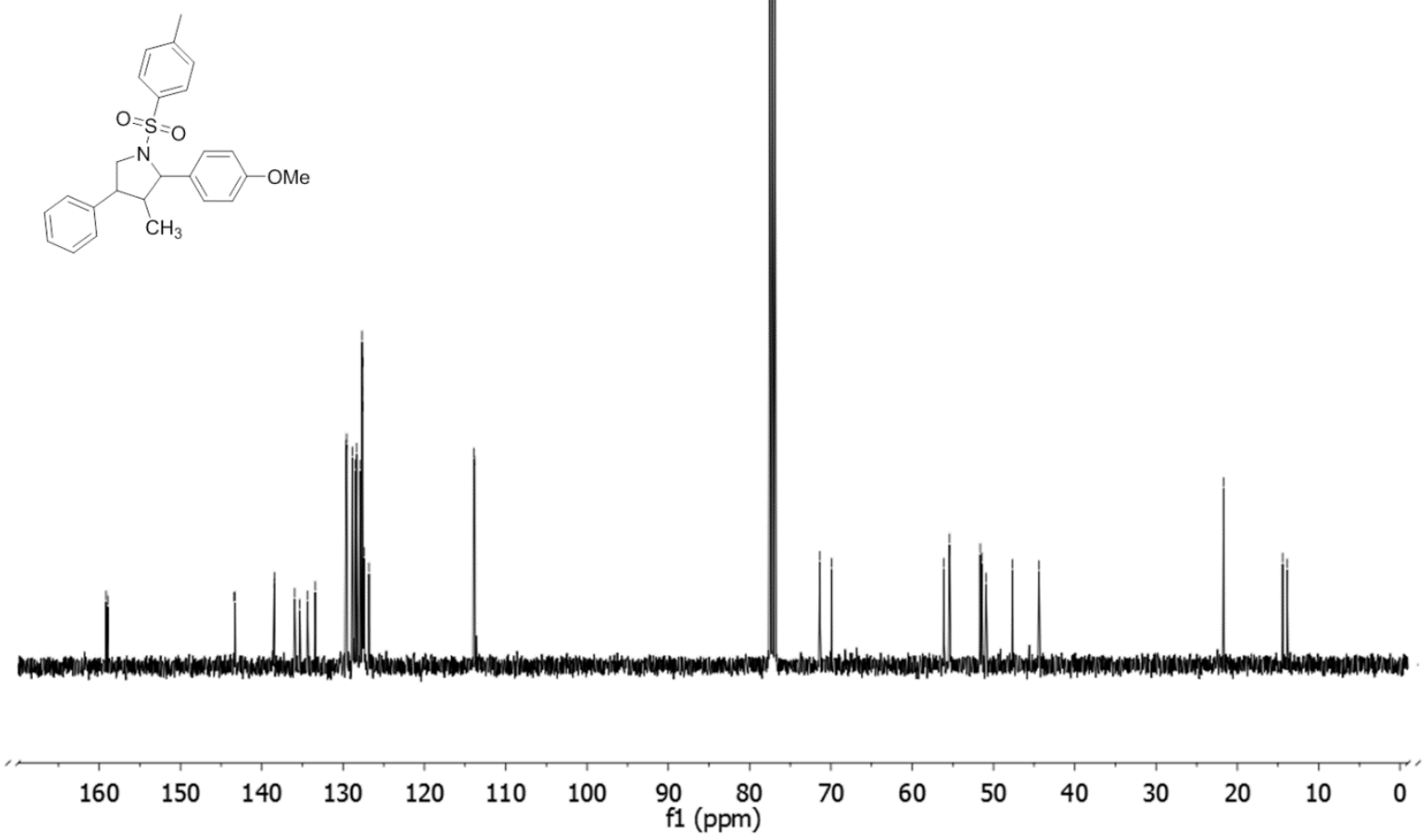


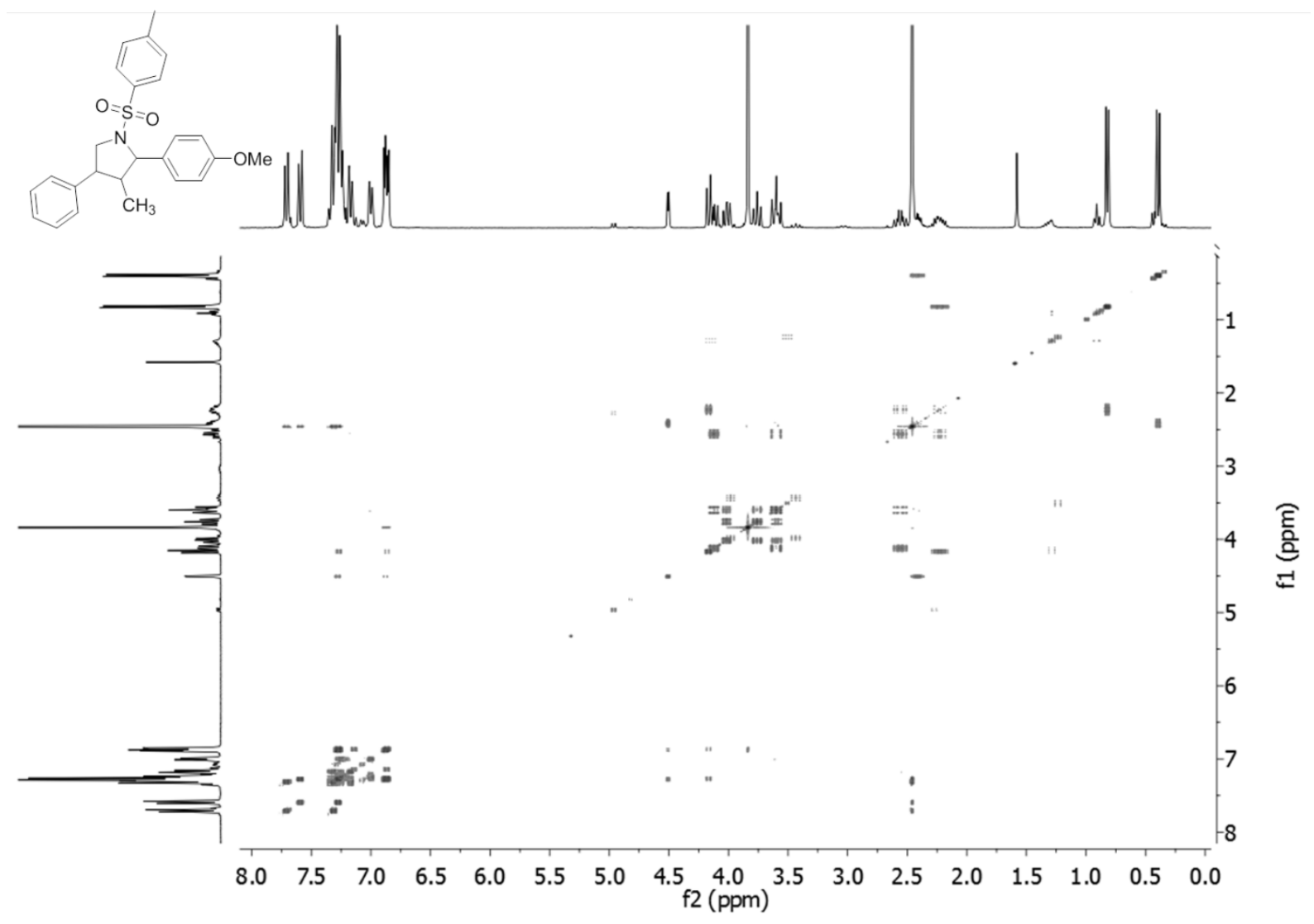




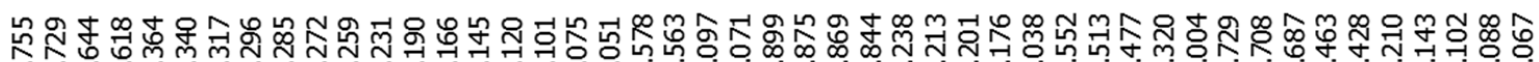

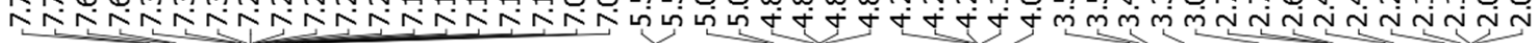

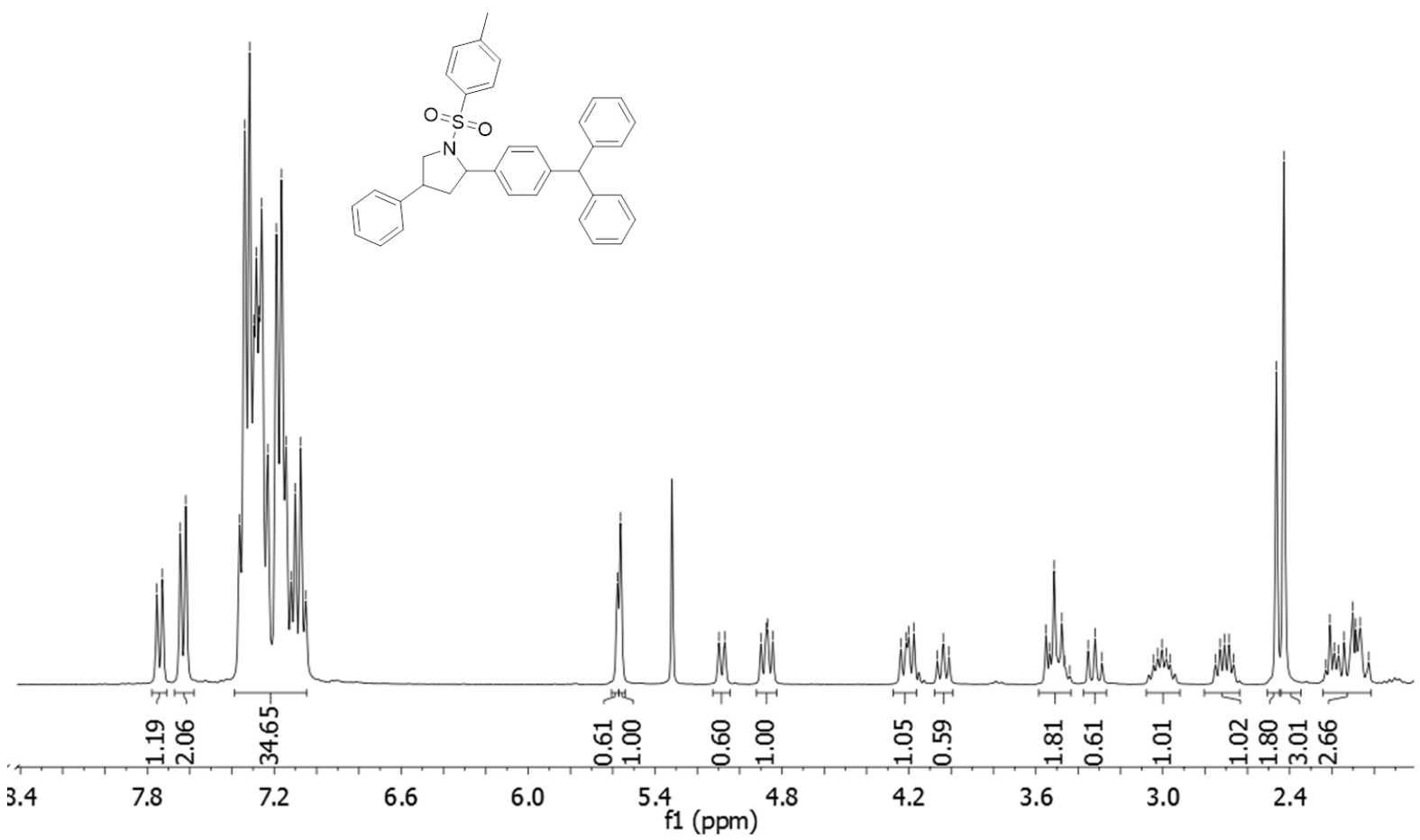

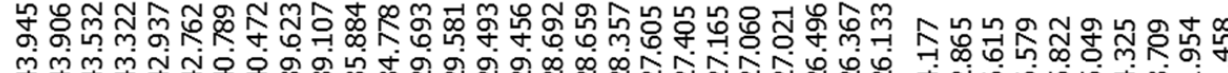

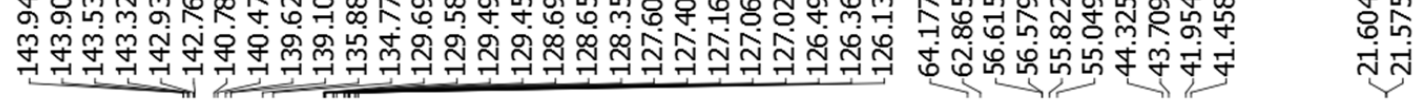
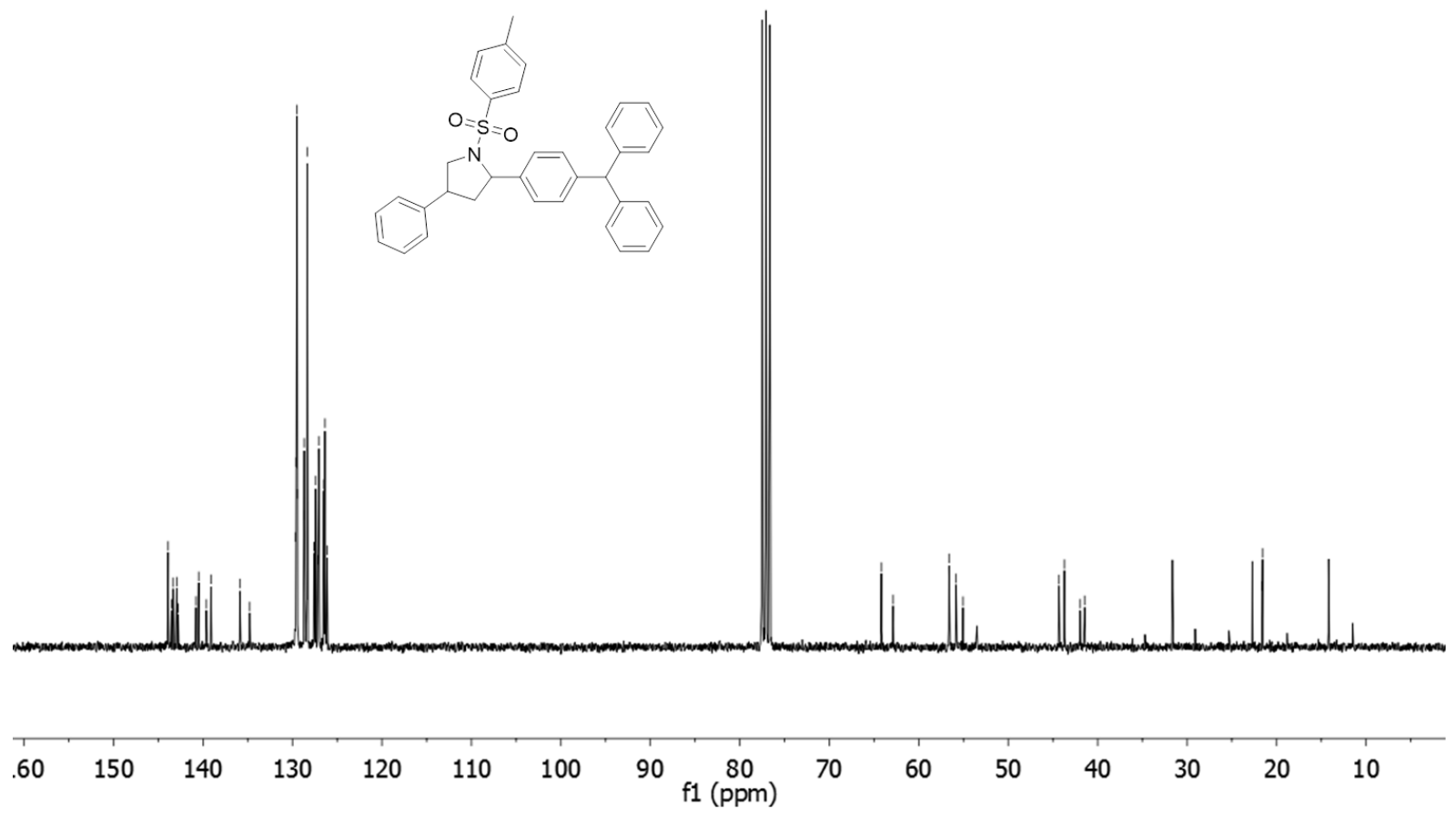


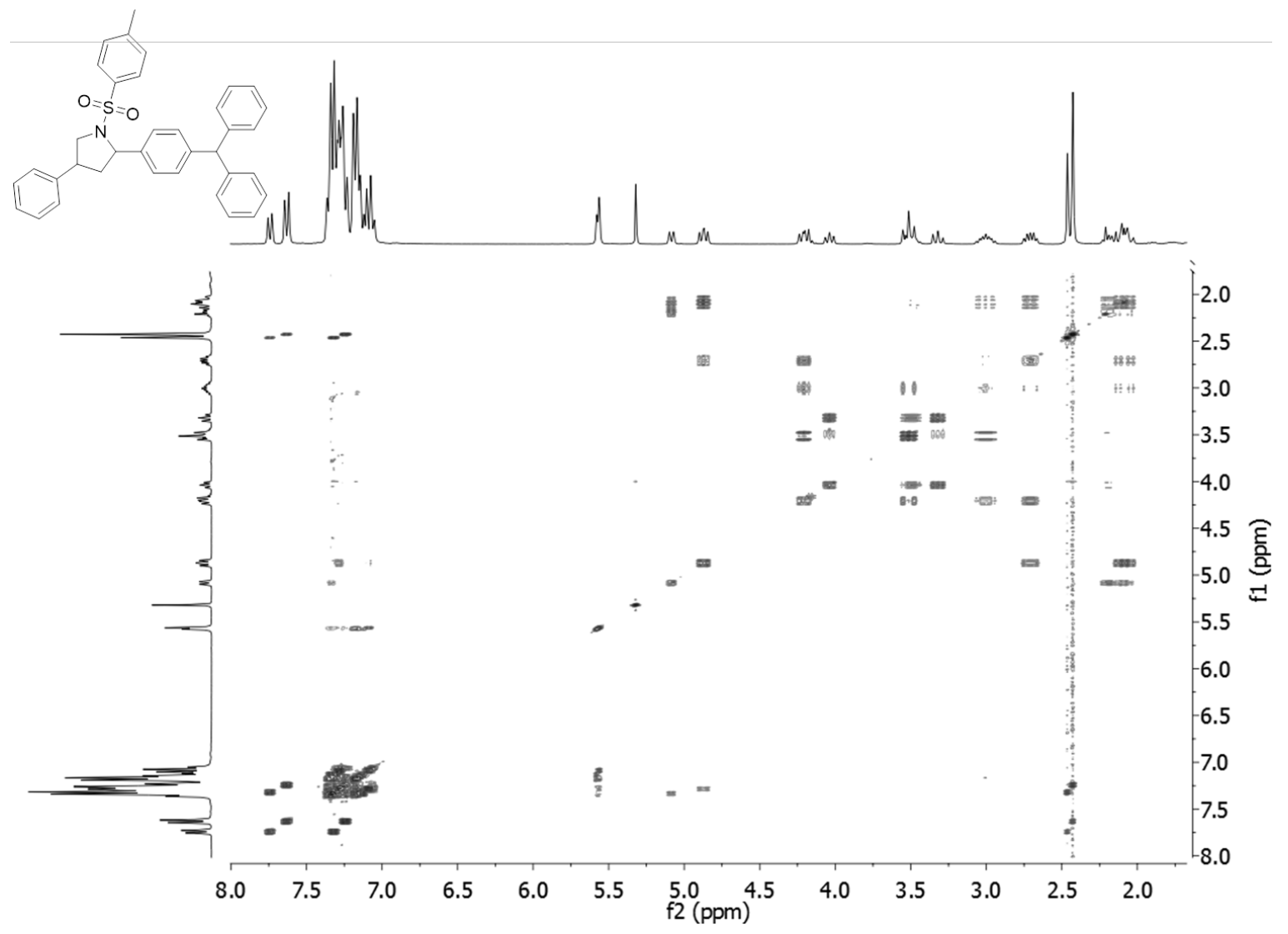



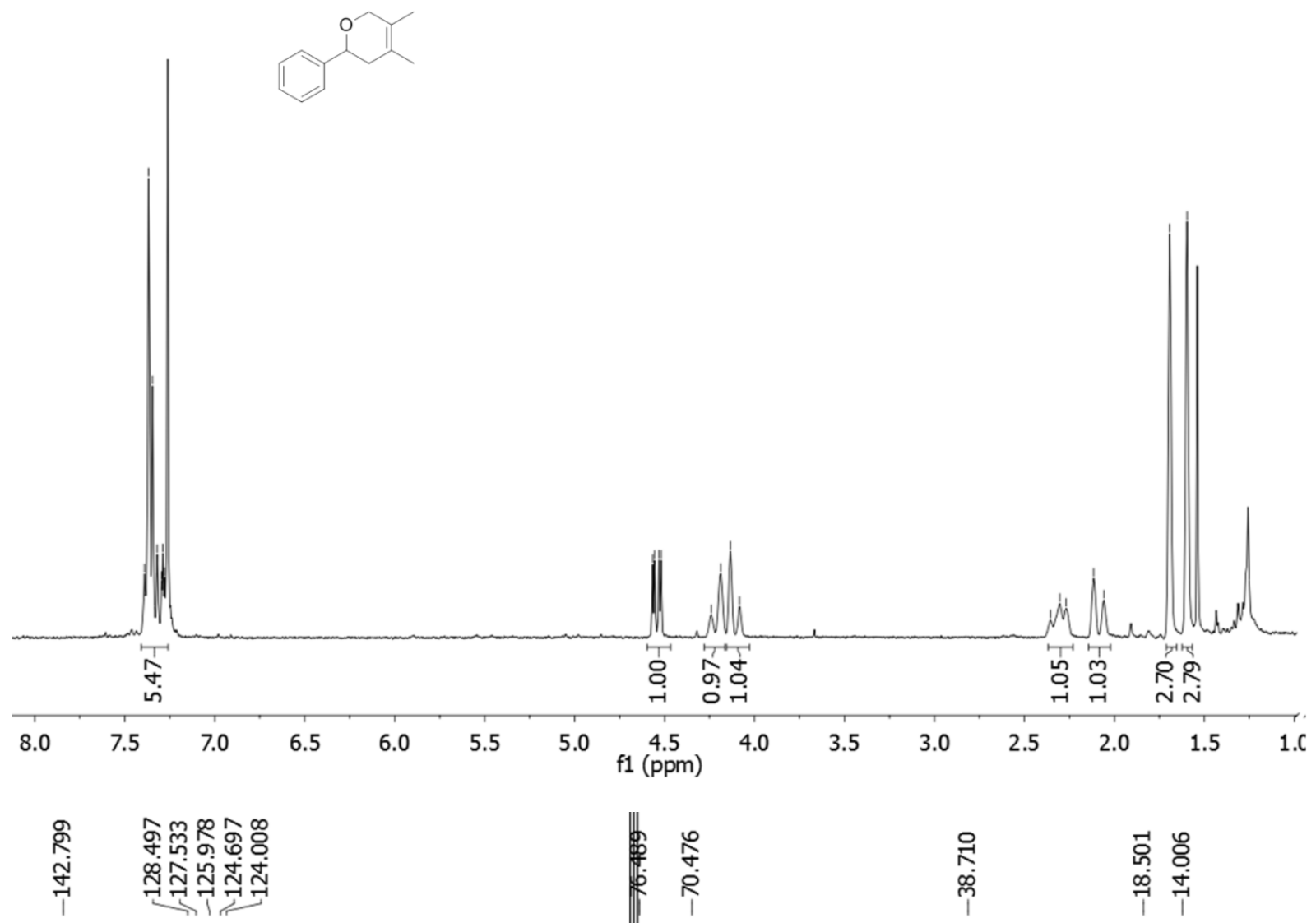

올

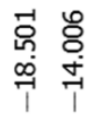
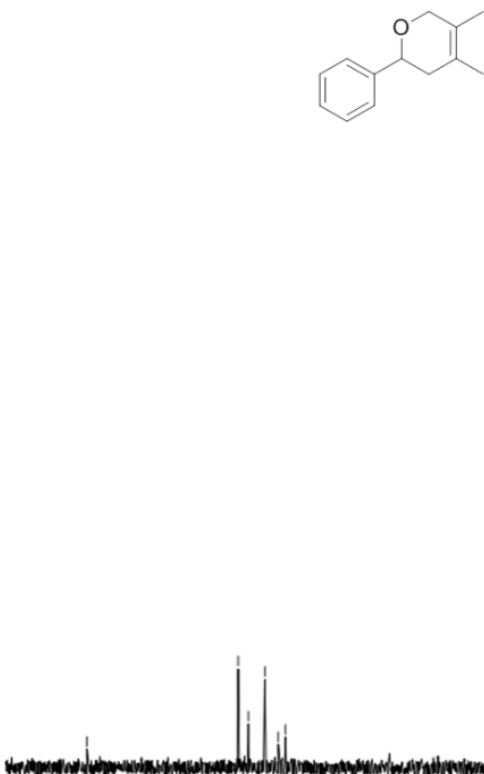

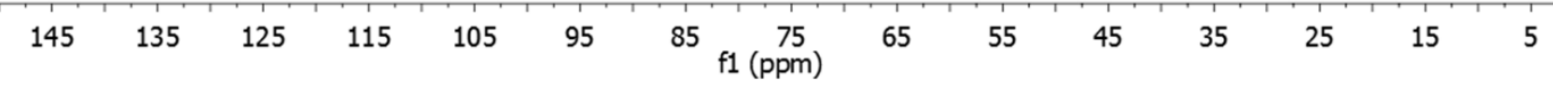




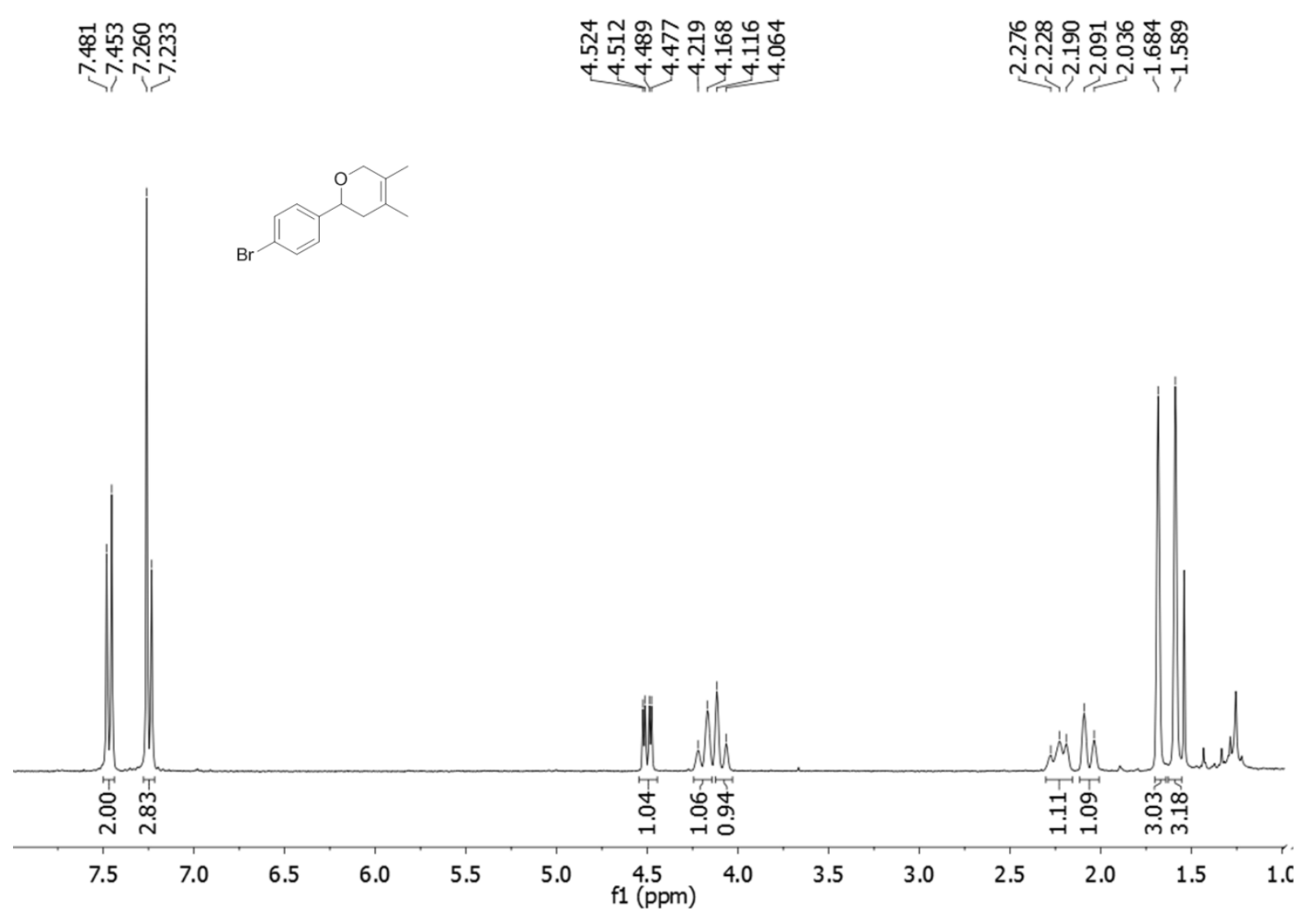

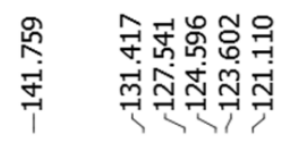

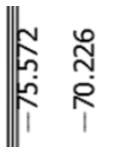

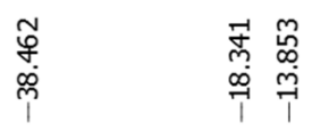

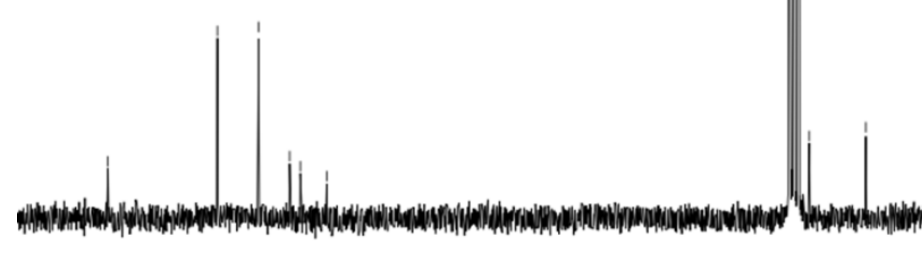

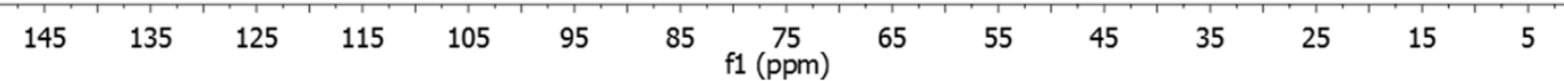




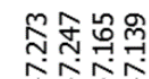

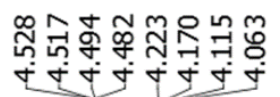

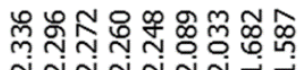

Nำกำ

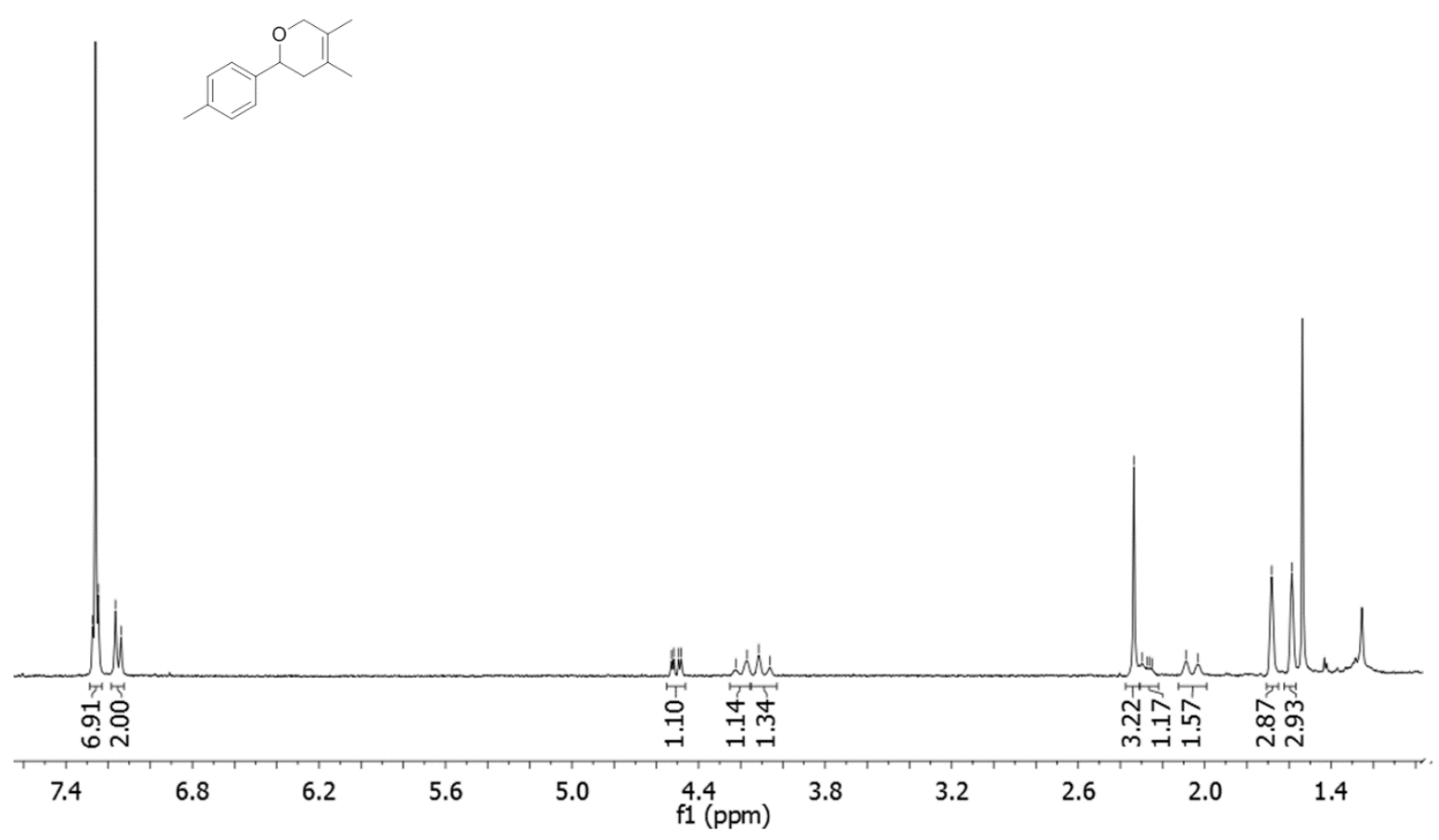

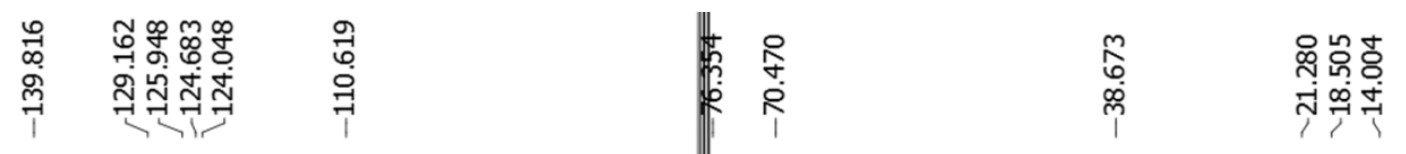

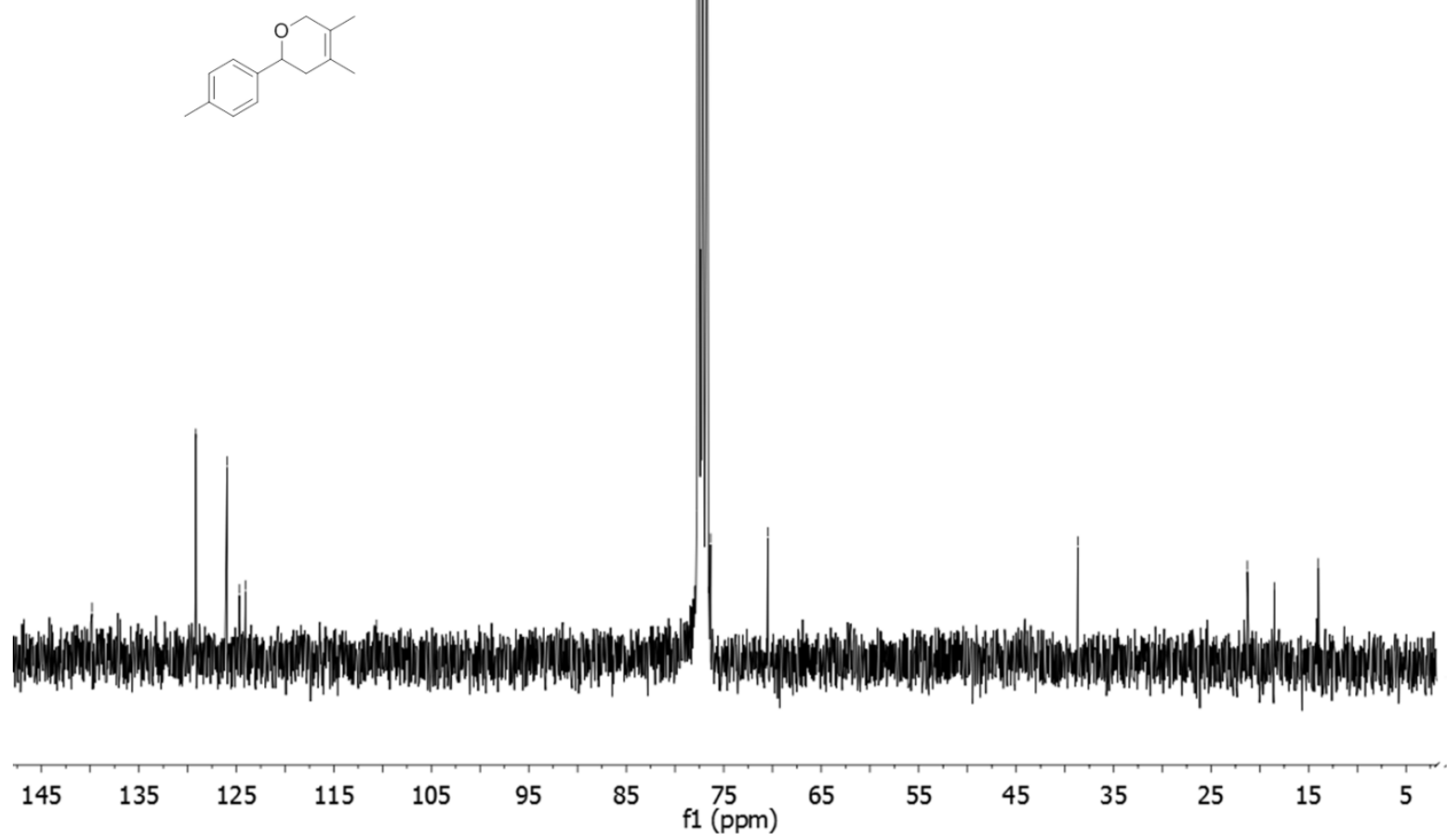




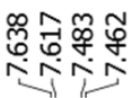

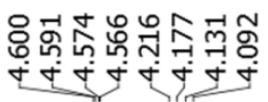

ลิి

NNNNiT
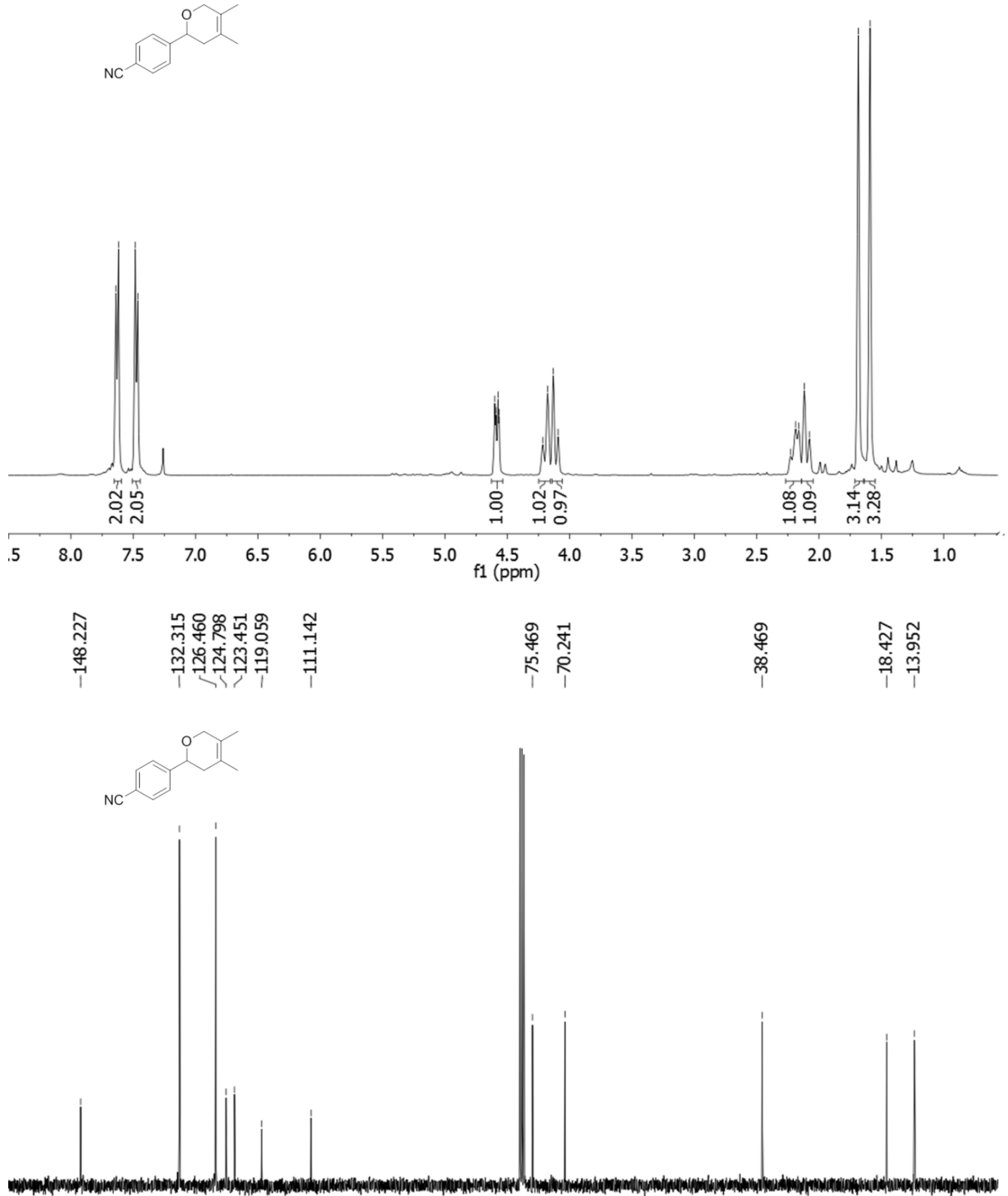

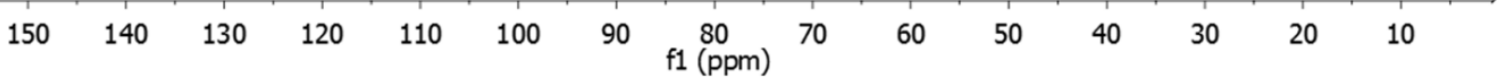




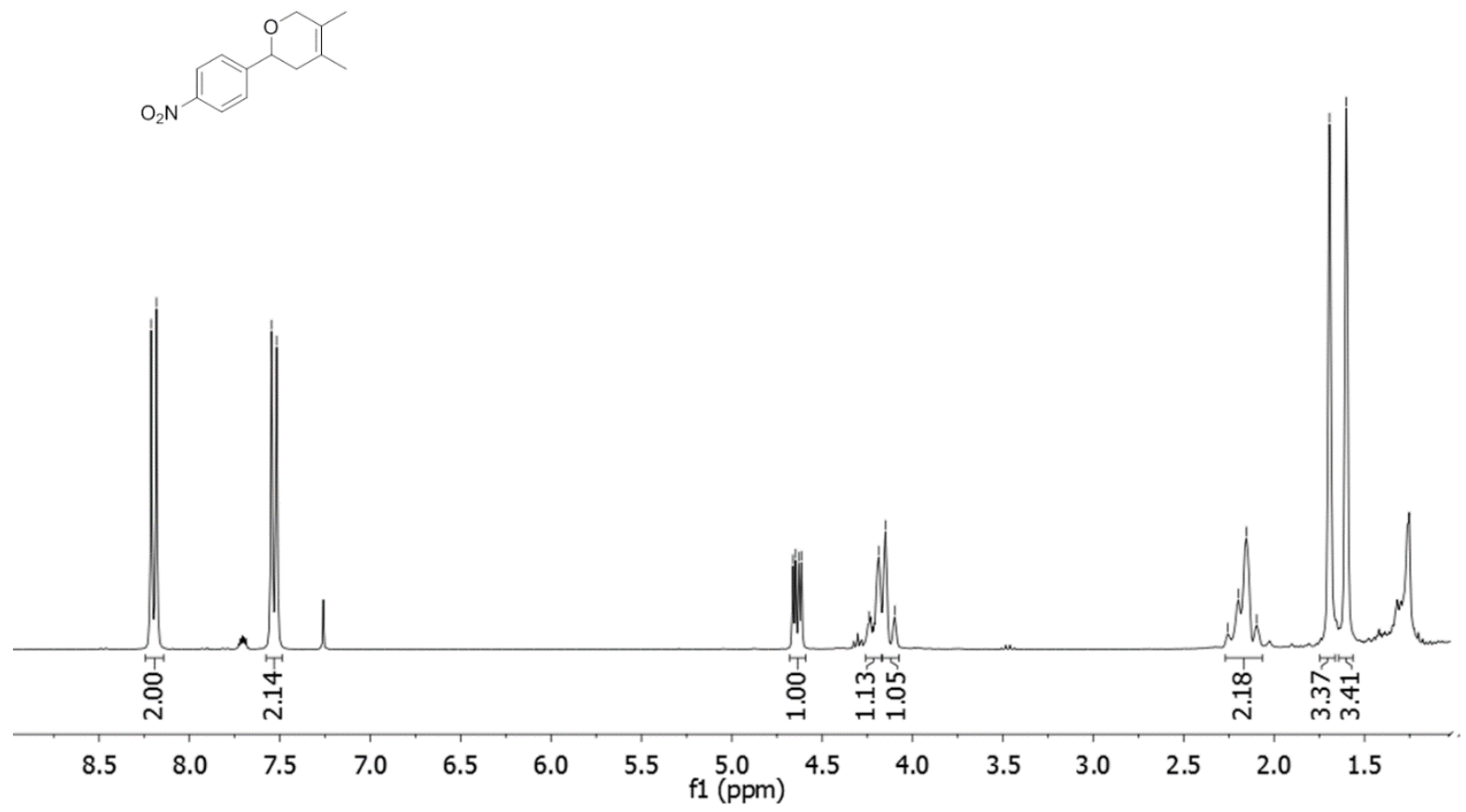

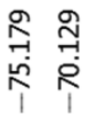

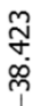

$\stackrel{\infty}{\circ}$

$\stackrel{\infty}{\rightarrow}$

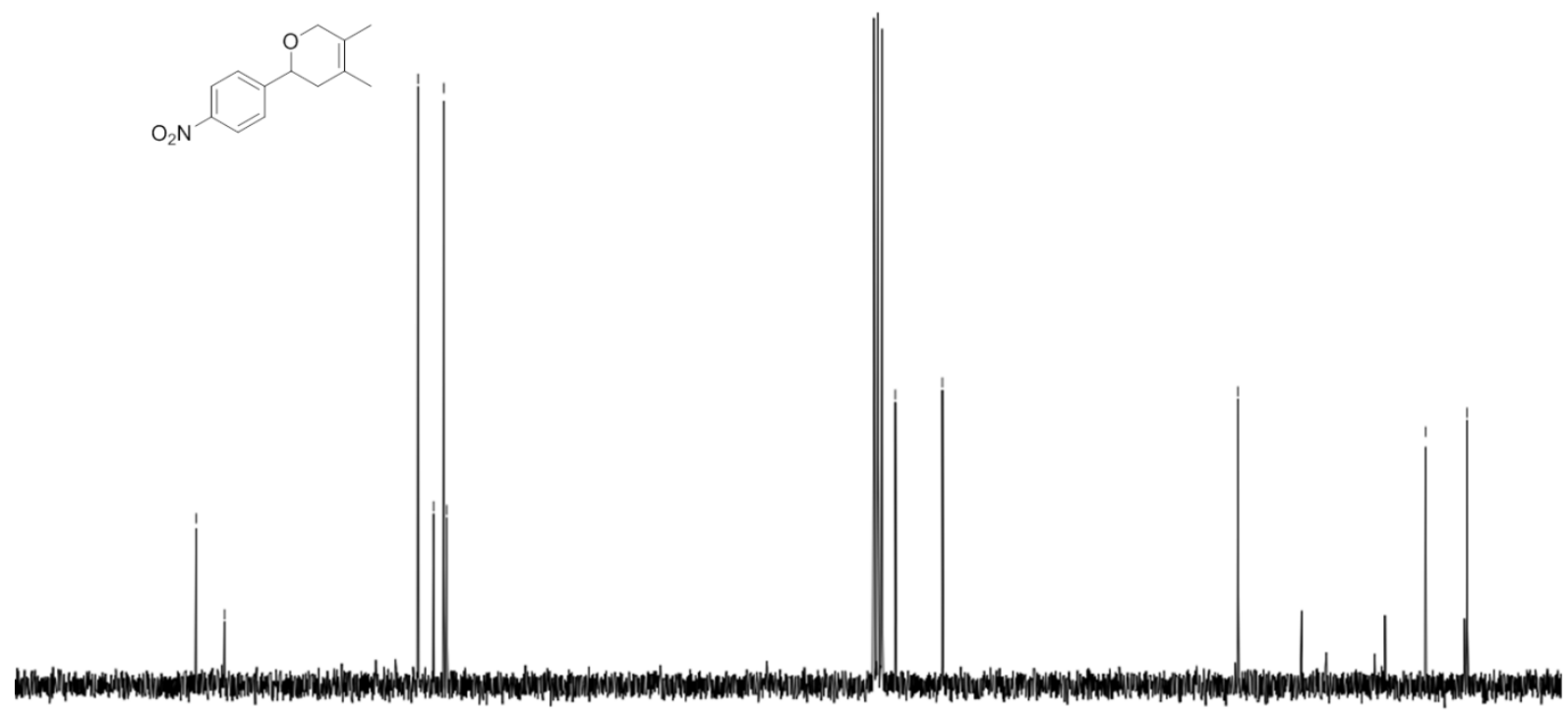

160

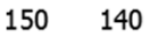

130

120

$\underset{f 1(p p m)}{90} \quad 70 \quad 60$

50

40

S- 90 - 


\section{Section S7 References}

(1) Bruker; Bruker AXS Inc.: Madison, Wisconsin, USA, 2010.

(2) Bruker; Data Reduction Software. Bruker AXS Inc.: Madison, Wisconsin, USA, 2009.

(3) Sheldrick, G. M.; Program for Empirical Absorption Correction: University of Göttingen, Göttingen, Germany, 2008.

(4) Farrugia, L. J. Journal of Applied Crystallography 1999, 32, 837.

(5) Sheldrick, G. M. Acta Crystallographica, Section A: Foundations of Crystallography 1990, A46, 467.

(6) Sheldrick, G. M.; Program for Structure Refinement: Univeristy of Göttingen, Göttingen, Germany, 1997.

(7) Sheldrick, G. M. Acta Crystallographica, Section A: Foundations of Crystallography 2008, A64, 112.

(8) Spek, A. L. Journal of Applied Crystallography 2003, 36, 7.

(9) McNitt, K. A.; Parimal, K.; Share, A. I.; Fahrenbach, A. C.; Witlicki, E. H.; Pink, M.; Bediako, D. K.; Plaisier, C. L.; Le, N.; Heeringa, L. P.; Griend, D. A. V.; Flood, A. H. Journal of the American Chemical Society 2008, 131, 1305.

(10) Kazmierczak, P.; Skulski, L. Synthesis 1998, 1721.

(11) Huang, C. Y.; Doyle, A. G. Journal of the American Chemical Society 2012, 134, 9541. 